\title{
Gefügekontrollierte Verwitterung natürlicher und konservierter Marmore
}

\author{
Dissertation \\ zur Erlangung des Doktorgrades \\ der Mathematisch-Naturwissenschaftlichen Fakultäten \\ der Georg-August-Universität zu Göttingen
}

vorgelegt von

Jörg Michael Rüdrich

aus Witzenhausen 
D 7

Referent: $\quad$ Prof. Dr. S. Siegesmund

Korreferent: $\quad$ Prof. Dr. R. Snethlage

Tag der Disputation: 03.07.2003 


\section{Kurzfassung}

In der vorliegenden Arbeit wurde die Gefügeabhängigkeit der Verwitterungsprozesse natürlicher und konservierter Marmore untersucht. Das Grundkonzept basiert auf dem Vergleich der Gesteinseigenschaften in fünf verschiedenen Zuständen: bruchfrisch, natürlich verwittert, künstlich verwittert, modellhaft konserviert und konserviert/künstlich verwittert. Dazu wurden polnische Marmore aus Prieborn, Grosskunzendorf, Kauffung und italienische Marmore aus Carrara, Lasa, Sterzing mit unterschiedlichen Gefügen untersucht. Grundlage für die Interpretation der Gefügeabhängigkeit der Entfestigungsprozesse bildet eine detaillierte Beschreibung aller relevanten Gefügemerkmale des Ausgangsmaterials. Die Charakterisierung der Gesteinszustände erfolgte mittels verwitterungssensibler petrophysikalischer Parameter: Porosität, Porengrößenverteilung, kapillare Wasseraufnahme, Ultraschallgeschwindigkeiten und Spaltzugfestigkeiten. Das Ausmaß der künstlichen Verwitterung wurde experimentell mittels Messungen der thermischen Dilatation bestimmt.

Für kristalline Marmore, die im unverwitterten Zustand nur sehr geringe Porositäten aufweisen, ist der initiale Verwitterungsprozess eine thermisch gesteuerte Mikrorissbildung. Ursache hierfür sind die anisotropen Dehnungseigenschaften des Calcit-Einkristalls, die bei Temperaturwechseln zu einem Spannungsaufbau im Gesteinsgefüge führen. Das Mikrorisswachstum erfolgt entlang von Gefügediskontinuitäten wie Korngrenzen, Spaltflächen und präexistierenden Rissen. Je nach Gefügeausbildung werden unterschiedliche Schwachstellen aktiviert. Dabei können die sechs untersuchten Marmore in drei Korngefügetypen untergliedert werden. Für die jeweiligen Typen ist ein entsprechendes Rissszenario festzustellen und damit auch ein vergleichbares petrophysikalisches Verhalten. Insbesondere für Ultraschalluntersuchungen, welche zur zerstörungsfreien Beurteilung des Erhaltungszustandes eingesetzt werden, müssen Rissanisotropien bei der Interpretation von Ultraschalldaten Berücksichtigung finden. Durch die fortschreitende Öffnung der Risse im weiteren Verwitterungsverlauf erhöht sich die Porosität und die Poren weiten sich. Der im unverwitterten Zustand dominierende Wassertransportmechanismus, die Dampfdiffusion, wechselt zu einem kapillaren Wassertransport. Folglich können weitere Schädigungsprozesse, wie z.B. die chemische Lösung an gesteinsinternen Kristallflächen, wirksam werden.

Für die Konservierung der Marmore wurden drei verschiedene Konservierungsverfahren angewandt: ein PMMA auf Lösungsmittelbasis $\left(\mathrm{PMMA}_{\mathrm{sol}}\right)$, ein vorkondensierter Polykieselsäureester (PKSE) und eine Acrylharzvolltränkung $\left(\mathrm{PMMA}_{\text {poly }}\right)$. Messungen der Porosität und der Porenradienverteilung belegen eine ausgeprägte Veränderung des Porenraums der konservierten Marmore. Beide Acrylharzverfahren zeigen eine Wiederherstellung der Kohäsion, was mittels Ultraschallanalysen nachgewiesen werden kann. Beim Überschreiten der jeweiligen Glasübergangstemperaturen von $\mathrm{PMMA}_{\text {sol }}$ und $\mathrm{PMMA}_{\text {poly }}$ tritt eine starke Veränderung des thermischen Dehnungsverhaltens auf. Die PKSE-Konservierung hat nur wenig Einfluss auf die thermische Dilatation, führt aber zu einer Stabilisierung des Korngefüges stark entfestigter Marmore.

Es kann festgestellt werden, dass die Rissentfestigung während der Verwitterung von Marmoren vom Gefüge kontrolliert wird. Somit ist für die Charakterisierung von Marmoren sowohl hinsichtlich ihres Erhaltungszustandes als auch für beabsichtigte Konservierungen eine materialspezifische Betrachtungsweise unabdingbar. 


\begin{abstract}
The present work deals with fabric controlled weathering processes of natural and consolidated marbles. The research bases on the comparison of rock properties in five different conditions: fresh, naturally weathered, artificially weathered, consolidated and consolidated/artificially weathered. Marbles with different rock fabrics were examined. The marbles originate from Prieborn, Grosskunzendorf, Kauffung (Poland) and Carrara, Lasa, Sterzing (Italy). An elementary requirement of the interpretation of fabric controlled deterioration processes is a detailed description of all important marble fabric properties. The characterisation of the different rock conditions was achieved by measurements of weathering-sensitive petrophysical parameters: porosity, pore size distribution, capillary water uptake, ultrasonic wave velocities and tensile strength. The extent of artificial decay was determined by experimental measurements of the thermal dilatation.
\end{abstract}

The weathering process of crystalline marbles starts with a thermally-induced microcrack growth, which is caused by a strong directional dependence of thermal dilatation properties of the calcite single crystal. This anisotropic expansion behaviour generates stresses in the marble during temperature changes. The microcrack growth occurs along fabric discontinuities, i.e. grain boundaries, cleavage planes and pre-existing cracks. According to the varying fabric properties a preferred activation of a respective discontinuity can be observed for the different marbles. The investigated marbles can be divided into in three grain fabric types, each with a specific crack scenario, and thus, similar petrophysical properties. Preferred orientation of crack systems must be considered when using ultrasonic investigations to determine the state of preservation of marbles. Due to the progressive crack growth during weathering the porosity increases and the pore size expands. Hence, the water transport mechanism changes from vapour diffusion to capillary water uptake. Subsequently, other damage processes can be activated, e.g. chemical solution.

For the consolidation of the marbles three approaches were adopted: a solution of polymethylmethacrylate $\left(\mathrm{PMMA}_{\mathrm{sol}}\right)$ dissolved in xylenes, a polysilicic acid ester (PKSE) and a total impregnation with a monomer methyl-methacrylate $\left(\mathrm{PMMA}_{\text {poly }}\right)$. Measurements of the porosity and effective pore size distribution evidence a strong modification of the pore space by consolidation. Both PMMA approaches show a reestablishment of cohesion which can be determined by ultrasonic velocity measurements. By reaching the respective glass transition temperatures of $\mathrm{PMMA}_{\text {sol }}$ and $\mathrm{PMMA}_{\text {poly }}$, a strong modification of thermal behaviour occurs. The PKSE consolidation of marbles only shows only minor changes of dilatation, but leads to a stabilisation of the grain fabric of strongly deteriorated marbles.

It can be summarized that the decay of marbles during weathering is caused by microcracks which are controlled by the marble fabric. Therefore, a rock-specific approach is indispensable for the characterisation of marbles as well as for their state of preservation and for the intended consolidation of material. 


\section{Inhaltsverzeichnis}

1 Einleitung .........................................................................................................................................

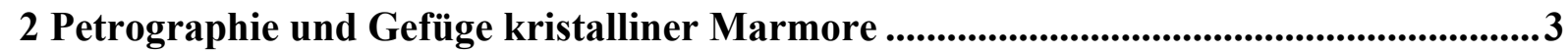

2.1 Beteiligte Mineralphasen und ihre Eigenschaften ..................................................... 3

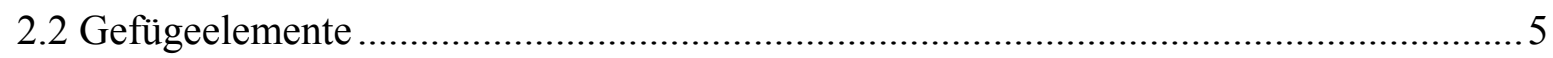

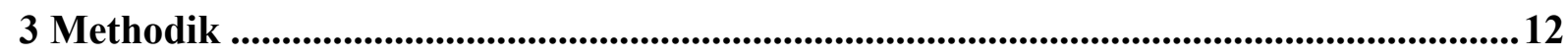

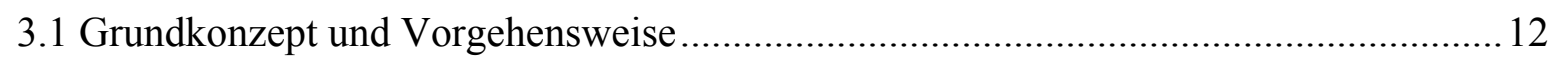

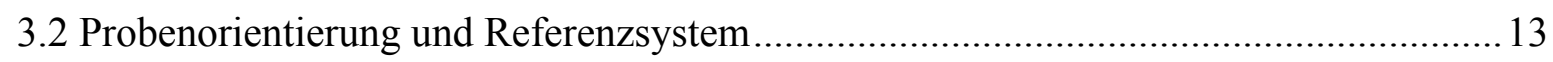

3.3 Petrographische und gefügekundliche Analysen.................................................... 13

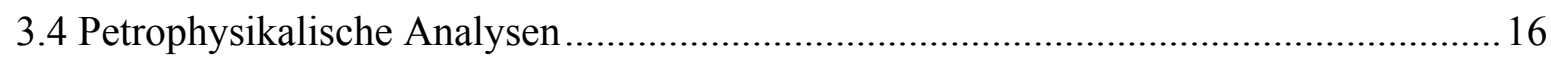

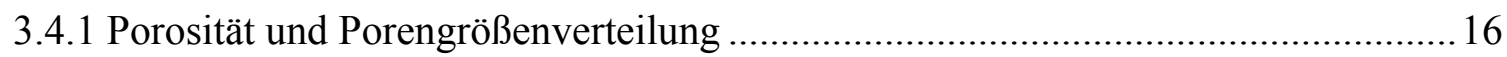

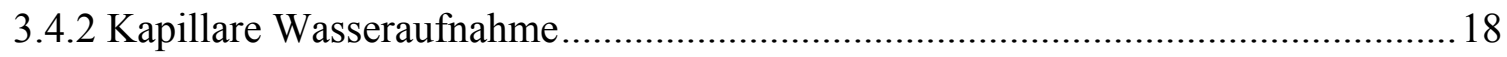

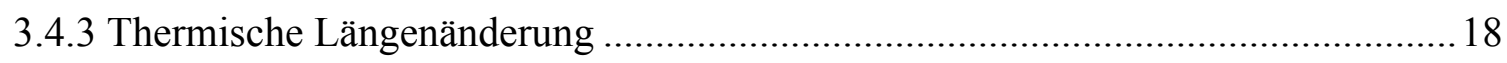

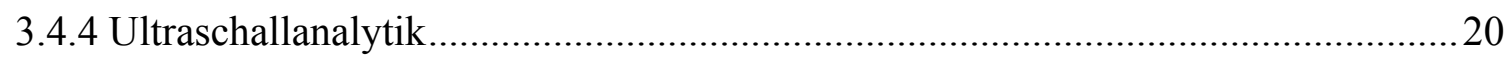

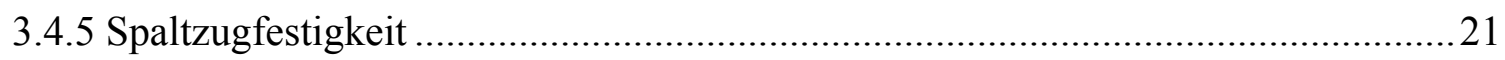

4 Untersuchte Marmore ..................................................................................................................22

4.1 Provenienz, Makrogefüge und Schadensphänomene ...............................................22

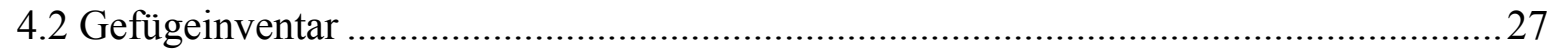

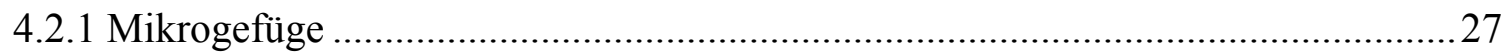

4.2.2 Verwitterungsgefüge (offene Mikrorisse) ....................................................... 38

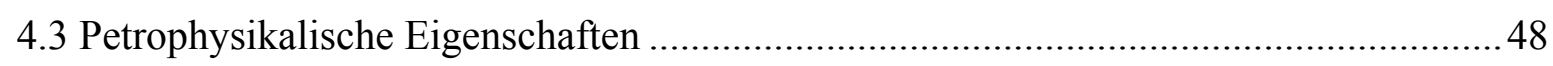

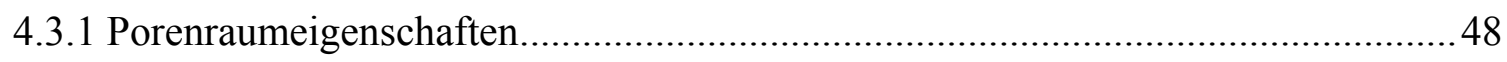

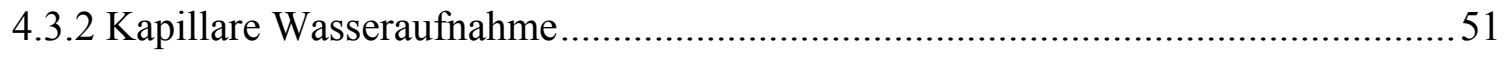

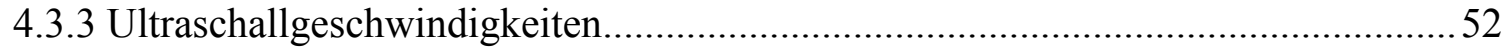

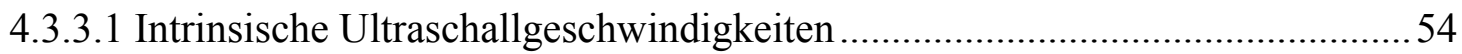

4.3.3.2 Rissbedingte Ultraschallgeschwindigkeitsabnahme.....................................55

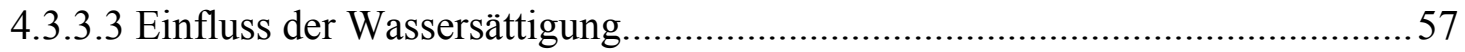

4.3.3.4 Quantifizierung der Vorzugsorientierung von Mikrorisssystemen ..................59

4.3.3.5 Zusammenhang zwischen $\mathrm{V}_{\mathrm{p}}$ und der Porosität ............................................. 61

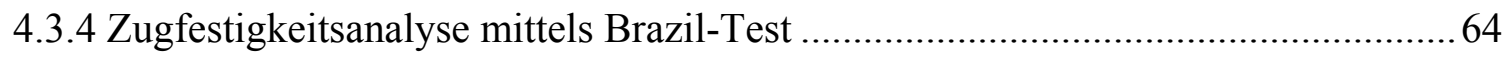

5 Gefügeabhängigkeit des thermischen Verwitterungsverhaltens von Marmoren..........69

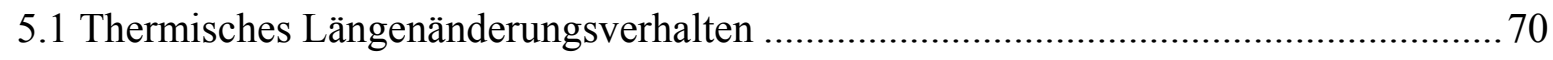


5.1.1 Anisotropie der thermischen Längenänderung von Marmor................................... 70

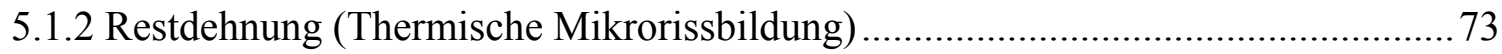

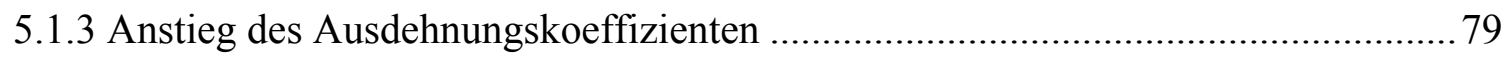

5.2 Änderung verwitterungssensibler petrophysikalischer Parameter .............................. 81

6 Modellhafte Konservierung verwitterter Marmore....................................................89

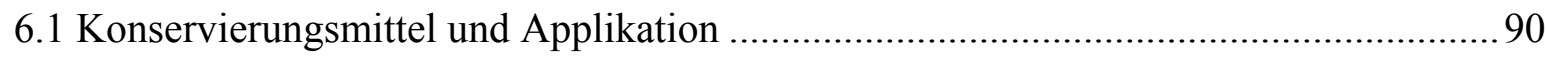

6.2 Vorkommen und Verteilung der Konservierungsmittel im Porenraum ........................92

6.3 Konservierungsbedingte Änderungen petrophysikalischer Parameter.........................94

7 Verhalten konservierter Marmore bei thermischer Beanspruchung.............................. 103

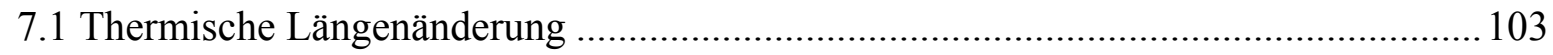

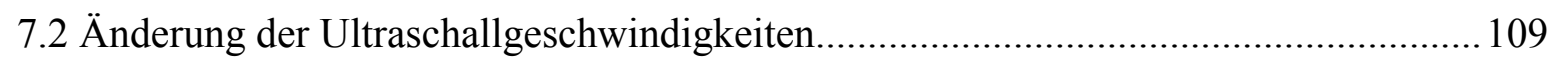

8 Überführung der Laborerkenntnisse ans Bauwerk ................................................112

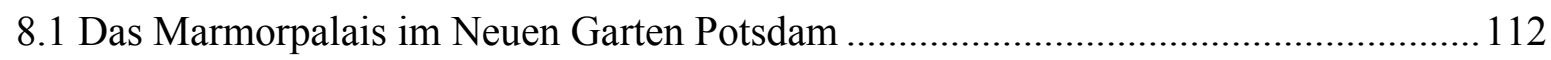

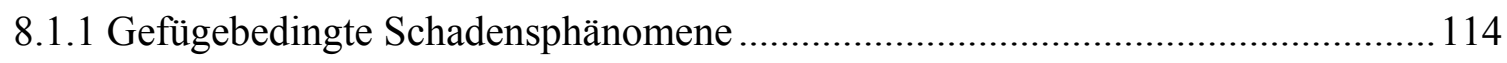

8.2 Fallstudie: Ultraschalltomographie an Marmorsäulen .............................................. 117

8.2.1 Ultraschalltomographische Untersuchungen ..................................................... 118

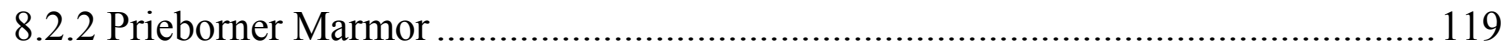

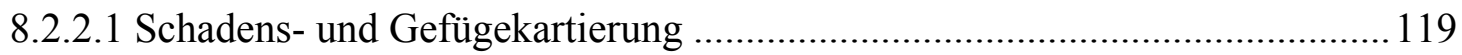

8.2.2.2 Tomographische Inversion der Ultraschalldaten........................................ 120

8.2.2.3 Labordaten vs. Felddaten............................................................................. 121

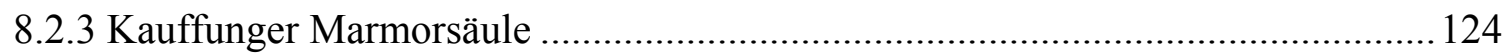

8.2.3.1 Schadenskartierung und Makro / Mikrogefüge ............................................. 124

8.2.3.2 Tomographische Inversion der Ultraschalldaten ....................................... 128

8.2.3.3 Labordaten vs. Felddaten..................................................................... 129

8.3 Fallstudie: Möglichkeiten des Einsatzes der Volltränkung vor Ort .......................... 130

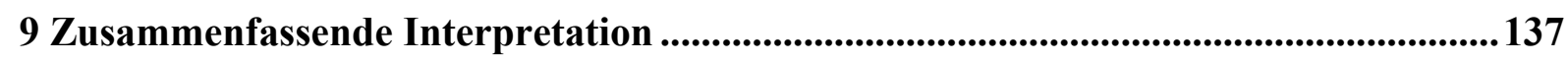

9.1 Gefügeabhängigkeit der thermomechanischen Entfestigungsprozesse von Marmoren138

9.2 Auswirkungen von Konservierungen auf die Marmoreigenschaften ........................... 142

9.3 Bedeutung der gefügeabhängigen Marmorverwitterung für die vor Ort Problematik . 145

9.4 Überführung der Erkenntnisse in den Umgang mit Marmor in der Praxis ................... 148

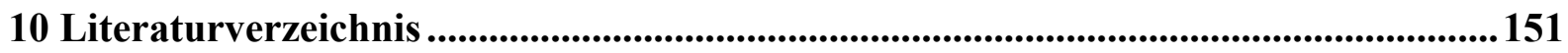




\section{Einleitung}

Der Witterung ausgesetzte Marmore erfahren durch physikalische, chemische und biologische Prozesse eine Gefügeauflockerung, die im Laufe der Zeit zur Zerstörung des Gesteins führt. Die Form und das Ausmaß der Verwitterung ist dabei neben der Exposition und den umweltbedingten Einflüssen auch wesentlich vom Gestein selbst abhängig.

Schäden an Bauteilen aus Marmor, die bei außenexponierten Objekten verbreitet auftreten, sind das körnige Zergrusen an der Gesteinsoberfläche sowie die Verformung von Marmorobjekten (vgl. GRIMM, 1999). Insbesondere letztere Schäden führen zu beeindruckenden Phänomenen, wie die konkave oder konvexe Wölbung von Marmorplatten als auch das Krümmen von Grabsteinen und Gedenkkreuzen. In den letzten Jahrzehnten erlangten diese Auswirkungen zunehmend wirtschaftliche Bedeutung. Das Auftreten entsprechender Schadensphänomene an Außenfassaden aus Marmor führte zu ihrem großflächigen Austausch (z.B. Amokko Gebäude, Chicago und Finlandia Halle, Helsinki) .

Die Hauptminerale des kristallinen Marmors (Calcit und Dolomit) besitzen eine ausgeprägte Richtungsabhängigkeit ihrer thermischen Ausdehnung. Dies kann bei entsprechenden klimatischen Verhältnissen ausschlaggebend für eine Entfestigung der Gesteinskomponenten und somit einer Gefügeauflockerung sein (GRIMM, 1984, GRIMM \& SchWARZ, 1985). Der Entfestigungsprozess, der sich mikroskopisch als Risswachstum an Korngrenzen und/oder kristallinternen Schwachstellen beschreiben lässt, ist zumindest im Initialstadium thermisch bedingt. Im Gegensatz zu vielen anderen Gesteinen, die im Wesentlichen eine Gefügeauflockerung in oberflächennahen Bereichen aufweisen, zeigen Marmore eine gesteinsdurchdringende Schädigung.

Ist das Gefüge erst einmal geschädigt erhöht sich mit fortschreitender Rissausbreitung die Porosität und bedingt dadurch eine verstärkte Einwirkung eindringender Feuchtigkeit (FrANZINI, 1995; WIDHALM et al., 1996). Resultierend können weitere Verwitterungsmechanismen, wie chemische Lösung, Frostsprengung und auch hygrische Dehnungsvorgänge initialisiert werden (s. Diskussion in POSCHLOD, 1989; GORALCZYK et al., 1988; KÖHLER, 1991). Insbesondere $\mathrm{CO}_{2}$ - und $\mathrm{SO}_{2}$-belastete Luft führt in Verbindung mit Wasser durch Calcitauflösung zu weiterer Schädigung.

Um die Lebensdauer von geschädigten Marmoren $\mathrm{zu}$ erhöhen, werden in der Praxis Konservierungen der betroffenen Bauteile mittels Steinfestigern durchgeführt. Obwohl umfangreiche Informationen über Konservierungen existieren, basiert die Auswahl eines geeigneten Imprägnierungsstoffes noch immer weitgehend auf empirischen Überlegungen. Ergibt ein Schutzmittel gute Ergebnisse mit einer Sorte von Gesteinen, wird dieses häufig auch auf andere Gesteinstypen ohne Überprüfung der Verträglichkeit angewandt. Einige der Faktoren, welche die Wirksamkeit eines Steinfestigers beeinflussen, sind bekannt, wie die Eindringtiefe und der Feuchtigkeitstransport durch den konservierten Stein. Hingegen wurden bisher nur wenige Untersuchungen bezüglich anderer wichtiger Faktoren durchgeführt, z.B. der Kompatibilität der thermischen Eigenschaften des Festigers mit der mineralogischen Zusammensetzung und dem Gesteinsgefüge (vgl. CLIFTON, 1980). 
Vorraussetzung für eine materialangepasste Durchführung von Konservierungsmaßnahmen ist die Kenntnis des Verwitterungs- bzw. Erhaltungszustandes des geschädigten Marmors. Beruhend auf der denkmalpflegerischen Zielsetzung kommen dabei zur Detektierung nur schadensfreie oder schadensarme Messmethoden in Frage (z.B. WIHR, 1980). Als geeignetes Verfahren hat sich dabei vor allem die Ultraschallanalytik herausgestellt. Aufgrund der in Marmoren häufig auftretenden Anisotropien und Heterogenitäten kommt es allerdings zu Überlagerungseffekten, die eine zweifelsfreie Identifikation der tatsächlichen Schäden erschweren (vgl. SIEGESMUND et al., 1999; WEISS et al., 2000; RUEDRICH et al., 2001b). Die genaue Lokalisierung entfestigter Zonen ist jedoch eine unverzichtbare Grundlage zur Entwicklung materialangepasster Sanierungsstrategien und zum Einsatz gezielter zustandsverbessernder Maßnahmen.

In der vorliegenden Arbeit wird versucht den Kenntnisstand über den bislang nur wenig untersuchten Einfluss des Gesteinsgefüges auf die thermomechanischen Entfestigungsprozesse von kristallinen Marmoren zu verbessern. Besondere Beachtung findet dabei die Frage ob und inwieweit die in Marmoren häufig entwickelten Gefügeanisotropien auch eine Richtungsabhängigkeit der Verwitterungsprozesse bedingen. Die Untersuchungen wurden an verschieden Calcit-Marmoren durchgeführt, die eine große Vielfalt an Gefügemerkmalen aufweisen (Textur, Kornformregelung usw.) und somit ein hohes Informationspotential besitzen.

In welcher Art und in welchem Umfang die Einbringung von Konservierungsmitteln in den Porenraum der Marmore deren Materialeigenschaften ändert ist ein weiterer Schwerpunkt. Dazu wurden drei „state of the art“ Konservierungsmittel bzw. Applikationsmethoden, welche unterschiedliche Wirkprinzipien repräsentieren, eingesetzt.

Ultraschalluntersuchungen stellen ein sehr wichtiges Hilfsmittel zur Analyse der verwitterungsbedingten Schäden von Marmoren dar. Daher sollte geprüft werden, ob und in welchem Umfang die in Marmoren vorhandenen intrinsischen und durch die Verwitterung erzeugten bzw. verstärkten Anisotropien bei der Interpretation von ultraschalldiagnostischen Untersuchungen Berücksichtigung finden müssen. 


\section{Petrographie und Gefüge kristalliner Marmore}

\subsection{Beteiligte Mineralphasen und ihre Eigenschaften}

Im Gegensatz zur steinverarbeitenden Industrie, wo fast alle dichten und polierfähigen Kalksteine als Marmore bezeichnet werden, verstehen die Geowissenschaften unter dem Begriff Marmor, das Produkt einer metamorphen Überprägung von Kalkstein. Nach der petrographischen Nomenklatur werden ausschließlich Gesteine als Marmor bezeichnet, die mehr als 50 Vol.-\% der Mineralphasen Calcit und/oder Dolomit beinhalten (vgl. WimMENAUER, 1985). Den Marmoren im eigentlichen Sinne mit $>90 \%$ Carbonat stehen die stärker quarz- und silikathaltigen Silikatmarmore mit $50 \%$ bis $90 \%$ Carbonat gegenüber. Im Folgenden wird der Begriff Marmor im Sinne der petrographischen Nomenklatur verwendet und beschreibt durch Regional- oder Kontaktmetamorphose überprägte Kalk- und Dolomitsteine.

Kieselige und mergelige Mineralkomponenten sowie organisches Material im karbonatischen Ausgangsgestein führen, je nach Metamorphosegrad, zur Ausbildung weiterer Mineralphasen als Nebengemengteile, z.B. Chlorit, Epidot, Granat, Muskovit, Biotit, Pyrit und Graphit. Diese Mineralphasen bilden auch die in vielen Marmoren auftretende Bänderung, die z.T. auch unregelmäßig („Marmorierung“) ausfallen kann. Weiterhin können auch feinverteilte Mineralphasen eine gewünschte Pigmentierung, wie z.B. Hämatit (rot), Chlorit und Serpentin (grün) erzeugen.

Das physikalische und mechanische Verhalten eines Gesteins wird durch die Eigenschaften der beteiligten Mineralphasen bedingt. Die wesentlichen Einkristalleigenschaften für die beiden Hauptkomponenten Calcit/Dolomit und weitere häufig in Marmoren vertretene Mineralphasen befinden sich in Tab. 2.1. Dabei kommt in der vorliegenden Arbeit der Richtungsabhängigkeit und der Intensität der thermischen Dilatation und den elastischen Eigenschaften des Calcit-Kristalls die wesentliche Bedeutung zu.

Tab. 2.1: Wesentliche Einkristalleigenschaften von Calcit und Dolomit sowie weiteren häufig in Marmor vertretenen Mineralphasen $\left({ }^{(1)}=\right.$ KLEBER, 1959; ${ }^{(2)}=$ FEI, 1995; ${ }^{(3)}=$ Tröger, 1967; ${ }^{(4)}=$ DANDEKAR, 1968; ${ }^{(5)}=$ MCSKIMIN et al., 1965; ${ }^{(6)}=$ VAUGHAN \& GUGGENHEIM, 1986).

\begin{tabular}{|c|c|c|c|c|c|}
\hline & Calcit & Dolomit & Quarz & Muskovit & Graphit \\
\hline $\begin{array}{l}\text { Chemische } \\
\text { Zusammensetzung }\end{array}$ & $\mathrm{CaCO}_{3}$ & $\mathrm{CaMg}\left(\mathrm{CO}_{3}\right)_{2}$ & $\mathrm{SiO}_{2}$ & $\begin{array}{c}\mathrm{KAl}_{2}\left[(\mathrm{OH})_{2} / \mathrm{Si}_{3}\right. \\
\left.\mathrm{AlO}_{10}\right]\end{array}$ & $\mathrm{C}$ \\
\hline Kristallsystem & trigonal & trigonal & trigonal & monoklin & hexagonal \\
\hline Dichte $\left[\mathrm{g} / \mathrm{cm}^{2}\right]$ & $2,71^{(3)}$ & $2,87^{(3)}$ & $2,65^{(3)}$ & $2,83^{(3)}$ & $2,27^{(3)}$ \\
\hline $\begin{array}{l}\text { Thermischer } \\
\text { Ausdehnungskoeffizient } \\
\alpha \times 10^{-6}\end{array}$ & $\begin{array}{l}\| \mathrm{c}=26,0^{(1)} \\
\| \mathrm{a}=-6,0^{(1)}\end{array}$ & $\begin{array}{c}\| \mathrm{c}=26,0^{(1)} \\
\| \mathrm{a}=6,0^{(1)}\end{array}$ & $\begin{array}{c}\| \mathrm{c}=9,0^{(1)} \\
\| \mathrm{a}=14,0^{(1)}\end{array}$ & $\begin{array}{c}\| \mathrm{c}=13,8^{(2)} \\
\| \mathrm{a}=9,9^{(2)}\end{array}$ & $\begin{array}{l}\| \mathrm{c}=26,0^{(1)} \\
\| \mathrm{a}=-1,2^{(1)}\end{array}$ \\
\hline $\begin{array}{l}\text { Kompressionswellen- } \\
\text { geschwindigkeit [km/s] }\end{array}$ & $\begin{array}{l}\min .=5,7^{(4)} \\
\max .=7,7^{(4)}\end{array}$ & $\begin{array}{l}\min .=6,3^{(4)} \\
\max .=8,5^{(4)}\end{array}$ & $\begin{array}{l}\min .=5,5^{(5)} \\
\max .=7,0^{(5)}\end{array}$ & $\begin{array}{l}\min =4,6^{(6)} \\
\max =8,1^{(6)}\end{array}$ & - \\
\hline
\end{tabular}

Die thermische Ausdehnung von Kristallen ist außer im kubischen Kristallsystem anisotrop. Dabei ist das trigonale Kristallsystem, zu dem auch der Calcit gehört, durch zwei Hauptdehnungsrichtungen gekennzeichnet. Damit bildet die Ausdehnung in diesen Systemen 
ein rotationssymmetrisches Ellipsoid, wobei die Rotationsachse mit der c-Achse zusammenfällt. Aufgrund der unterschiedlichen Expansion in die zwei Hauptdehnungsrichtungen, ändert sich bei Erwärmung eines trigonalen Kristalls nicht nur das Volumen sondern auch seine Form. So wird sich aus einer Kristallkugel bei Erwärmung ein Ellipsoid bilden.

In der Literatur werden unterschiedliche Werte des thermischen Ausdehnungskoeffizienten für den Calcit-Einkristall diskutiert. Die meisten Werte fokussieren dabei auf Temperaturbereiche oberhalb $100{ }^{\circ} \mathrm{C}$, die für den natürlichen Klimabereich nur bedingt relevant sind. Des Weiteren werden von einigen Autoren lineare, von anderen nichtlineare Ausdehnungskoeffizienten aufgeführt. Z.B. gibt FEI (1995) für die Richtung parallel zur cAchse einen nichtlinearen sehr stark ansteigenden thermischen Expansionskoeffizienten an, der bei $25{ }^{\circ} \mathrm{C} \alpha_{11}=13,1 \cdot 10^{-6} \mathrm{~K}^{-1}$ und bei $90{ }^{\circ} \mathrm{C} \alpha_{11}=19,2 \cdot 10^{-6} \mathrm{~K}^{-1}$ beträgt (vgl. Abb. 2.1). Parallel zu den a-Achsen führt er hingegen einen linearen Wert von $\alpha_{22}=\alpha_{33}=-3,2 \cdot 10^{-6} \mathrm{~K}^{-1}$ an.

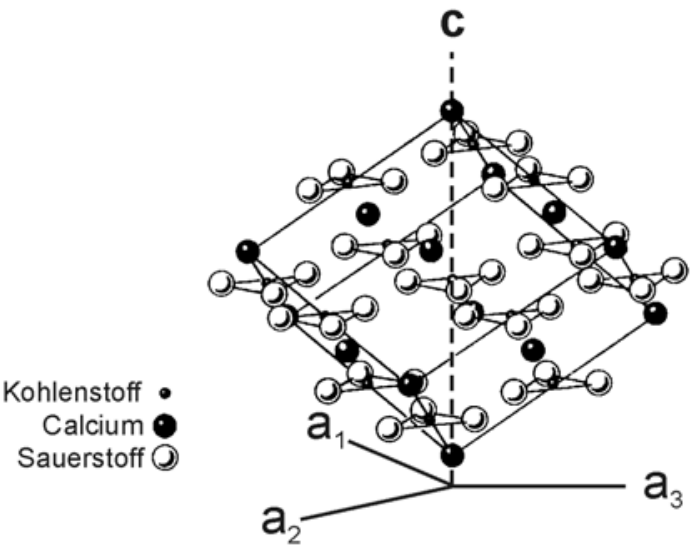

a)

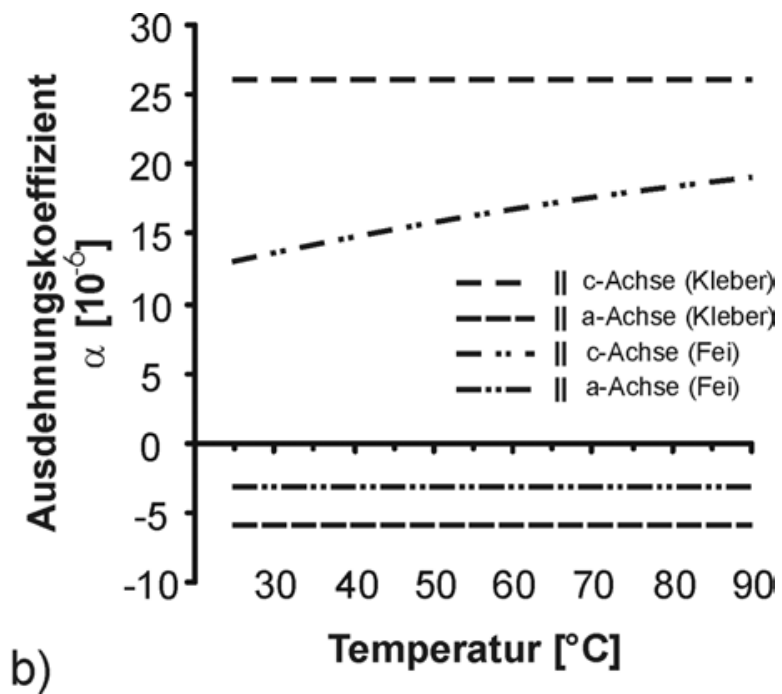

Abb. 2.1: Beziehung zwischen kristallographischen und physikalischen Eigenschaften des CalcitEinkristalls; a) Spaltrhomboeder des Calcit-Kristalls und b) thermischer Ausdehnungskoeffizient bei Temperaturänderung nach KLEBER (1959) und FEI (1995).

Nach KLEBER (1959) weisen beide Kristallrichtungen einen linearen Ausdehnungskoeffizienten von $\alpha_{11} 26 \cdot 10^{-6} \mathrm{~K}^{-1}$ parallel zur c-Achse und $\alpha_{22}=\alpha_{33}=-6 \cdot 10^{-6} \mathrm{~K}^{-1}$ parallel zu den a-Achsen auf (s. Abb. 2.1). Weitere Angaben befinden sich in SkInNER (1966) und SAGE (1988). Im folgenden werden für schematische Abbildungen die stark anisotropen linearen Werte von KLEBER (1959) verwendet. Bei direkten Vergleichen mit experimentell ermittelten Ausdehnungskoeffizienten fließen die verschiedenen Angaben mit in die Diskussion ein.

Neben der extremen Richtungsabhängigkeit der thermischen Dilatation weist der CalcitEinkristall ebenfalls stark anisotrope Ultraschallgeschwindigkeitsvariationen auf (s. Abb. 2.2). Dabei können für Kompressionswellen parallel zur c-Achse relativ langsame Geschwindigkeiten mit $5,7 \mathrm{~km} / \mathrm{s}$ und sehr hohe von $7,7 \mathrm{~km} / \mathrm{s}$ parallel $\mathrm{zu}$ den a-Achsen festgestellt werden (DANDEKAR, 1968). 

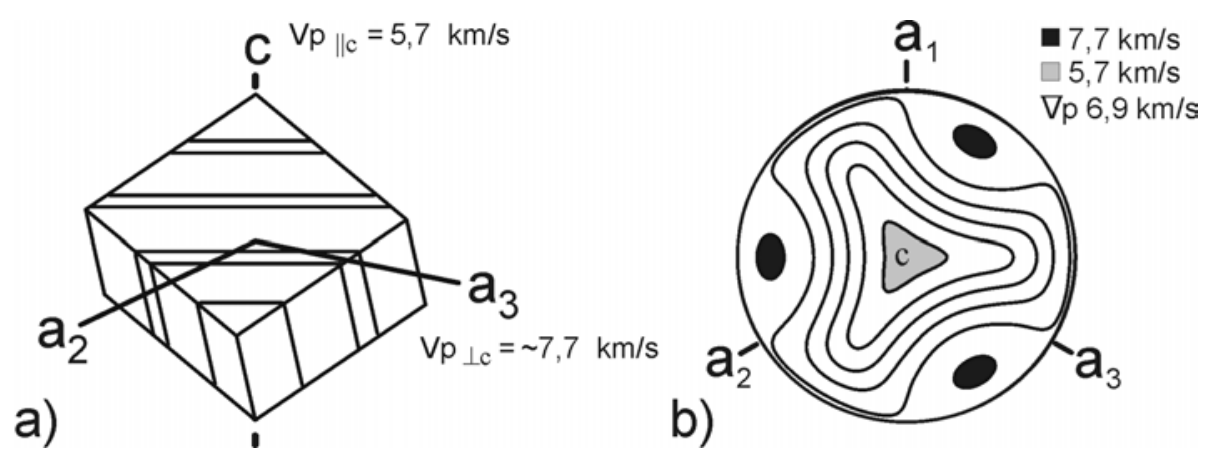

Abb. 2.2: Beziehung zwischen kristallographischen und physikalischen Eigenschaften des CalcitEinkristalls; a) Kompressionswellengeschwindigkeiten parallel $\mathrm{zu}$ den jeweiligen Kristallachsen sowie b) Geschwindigkeitsverteilung (P-Welle) in einem Calcit-Einkristall (Schmidt'sches Netz, untere Halbkugel; modifiziert nach JOHNSON \& WENK, 1985).

\subsection{Gefügeelemente}

Der Begriff „Gefüge“ wird im allgemeinen verwendet, um die räumliche Anordnung der beteiligten Komponenten sowie ihre Nachbarschaftsbeziehungen untereinander zu beschreiben (Form, Größe, Orientierung, Art des Kontakts; vgl. SANDER, 1930). Von verschiedenen Autoren existieren dazu erweiterte Definitionen, die sich spezieller auf das zu beschreibende Gestein konzentrieren. So umfasst der „Gefüge“-Begriff nach SIEGESMUND (1996), der im wesentlichen auf strukturgeologischen und petrophysikalischen Arbeiten an metamorphen Gesteinen basiert, u.a. die Regelung nach dem Kristallgitter (Textur) und nach der Kornform (Formregelung) der Minerale, die Verteilung, den Zustand und die Orientierung von Rissen, die Form, die Größe und die räumliche Verteilung der einzelnen Mineralkörner und deren Beziehung untereinander.

Generell resultieren die Material- und Verwitterungseigenschaften von Naturwerksteinen aus der stofflichen Zusammensetzung und den Gefügeeigenschaften. Dabei besitzen Natursteine sehr heterogene und anisotrope Gefüge, die auf ihre vielgestaltige und oft mehrphasige Entstehung (Sedimentation, Diagenese, Metamorphose, Deformation) über längere geologische Zeiträume zurückzuführen sind.

Heterogenitäten betreffen Eigenschaften, die unregelmäßig im Gestein verteilt und daher schwer zu prognostizieren sind. Sie können als punktuelle „Störstellen“ wirksam werden und sind sowohl deterministischer als auch chaotischer Natur. Hierbei kann es sich z.B. um chemische Variationen in einem strukturell homogenen Mineral, um „Fremdminerale“, Bereiche stärkerer Durchfeuchtung oder um abnorme Poren handeln. Mechanische Bedeutung besitzen diese „Störstellen“ vor allem als Orte, an denen sich Spannungsspitzen aufbauen (z.B. durch heterogene Wärmedehnung) und Verwitterungsprozesse verstärkt einsetzen können.

Anisotropien sind planare und lineare Vorzeichnungen, die sich meist durch homogene Anordnung von Mineralkörnern, Kristallgittern oder Mikrorissen (auch Poren) in bevorzugten Richtungen ergeben. Dementsprechend besitzen auch Gesteine in der Regel ein anisotropes Materialverhalten, das durch diese Vorzeichnungen diktiert wird (z.B. Spaltbarkeit, richtungsabhängige Korrosion etc.). 
Kristalline Marmore können ein enorm umfangreiches Gefügeinventar aufweisen. Hinsichtlich der Verwitterung von Marmor und damit auch der Güte des Gesteins scheinen jedoch vor allem spezifische Gefügeelemente die petrophysikalischen Eigenschaften bevorzugt $\mathrm{zu}$ kontrollieren und/oder deutlich $\mathrm{zu}$ beeinflussen. Die wesentlichen Gefügeeigenschaften sind im folgenden detaillierter aufgeführt um auch die in dieser Arbeit verwendete Terminologie zu definieren.

\section{- Korngefüge}

Das Korngefüge gehört zu den wesentlichen Gefügeeigenschaften eines Marmors und wird über die Parameter der Korngröße, der Kornform, der Korngrenzengeometrie und der Kornformregelung beschrieben. Allgemein zeichnen sich kristalline Marmore durch ein granoblastisches bis xenoblastisches Korngefüge aus.

\section{- Korngröße}

Die Korngröße ist für alle Gesteine ein wesentliches Merkmal und gibt Informationen über die Genese des Materials. Für das Verwitterungsverhalten sind die wichtigsten Eigenschaften die eigentliche Korngröße und der Anteil bestimmter Korngrößen am Gesamtkorngrößenspektrum. Die Bedeutung der Korngröße bzw. die Korngrößenverteilung für das Materialverhalten begründet sich vor allem darin, dass sie die Häufigkeit und die Verteilung von Korngrenzen bedingen. Dabei stellen die Korngrenzen besondere Schwachstellen für mechanisch ansetzende Kräfte dar. In dieser Arbeit folgt die Angabe der Korngrößen, der Skala für kristalline Gesteine von TEUSCHER (s. Tab. 2.2).

Tab. 2.2: Korngrößenskala für kristalline Gesteine nach TEUSCHER, aus WIMMENAUER (1985, S. 5).

\begin{tabular}{|l|c|c|}
\hline Bezeichnung & $\begin{array}{c}\text { Korndurch- } \\
\text { messer in mm }\end{array}$ & $\begin{array}{c}\text { Kornzahl pro } \\
\mathbf{c m}^{2}\end{array}$ \\
\hline riesenkörnig & $>33$ & $<<1$ \\
großkörnig & $33-10$ & $<1$ \\
grobkörnig & $10-3,3$ & $1-10^{1}$ \\
mittelkörnig & $3,3-1,0$ & $10^{1}-10^{2}$ \\
kleinkörnig & $1,0-0,3$ & $10^{2}-10^{3}$ \\
feinkörnig & $0,3-0,1$ & $10^{3}-10^{4}$ \\
sehr feinkörnig & $0,1-0,01$ & $10^{4}-10^{6}$ \\
dicht & $<0,01$ & $>10^{6}$ \\
\hline
\end{tabular}

Die Korngrößenverteilung von kristallinen Gesteinen kann gleichkörnig (equigranular) oder auf unterschiedliche Art ungleichkörnig sein (Abb. 2.3; vgl. MoORE, 1970). Ist der Korngrößenübergang unstetig wird sie als inequigranular oder auch hiatal bezeichnet und beschreibt eine bimodale Verteilung der Korngrößenklassen. Variiert die Korngröße stetig von einem Maximum zu einem Minimum wird die Verteilung hingegen als seriate oder serial bezeichnet (vgl. PAsschier \& Trouw, 1996). 


\section{- Kornform und Korngrenzengeometrie}

In kristallinen Marmoren besitzen die Kristalle fast ausschließlich xenomorphe Kornform, da die Kristalle mehr oder weniger gleichzeitig unter gleichberechtigten Kristallpartnern gewachsen sind. Die häufig in rekristallisierten Marmoren zu beobachtenden polygonalen Kornformen stellen nur eine Minimierung der Oberflächen durch Prozesse wie die Korngrenzenflächenreduktion (,grain boundary area reduction“) dar. Bezüglich der geometrischen Beschreibung von Kornaggregaten kristalliner Marmore kann eine grobe Einteilung in polygonal (aneinander liegende Körner) und interlobat (ineinander greifende Körner) erfolgen (s. Abb. 2.3; vgl. MoORE, 1970).
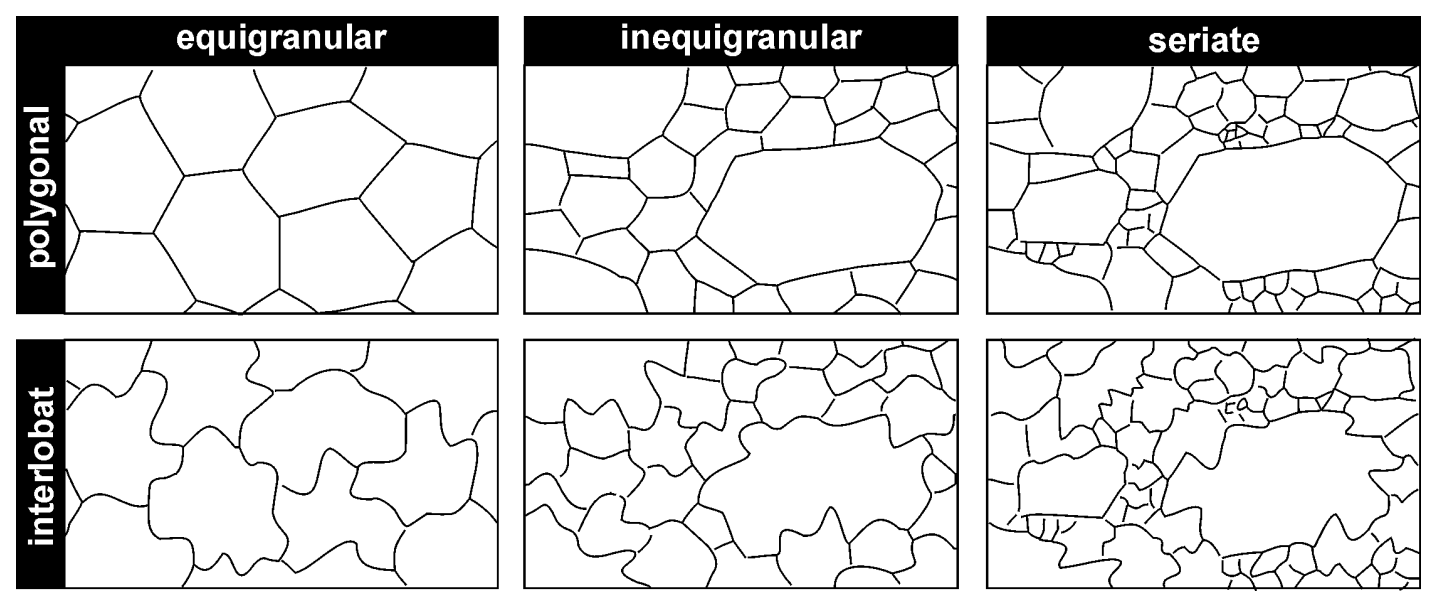

Abb 2.3: Korngrößenverteilung und Kornaggregatverhältnisse in kristallinen Gesteinen nach Moore (1970), verändert nach Passchier \&Trouw (1996).

Für die Interpretation gefügeabhängiger Verwitterungsprozesse von Marmoren ist es in den meisten Fällen jedoch sinnvoll die Korngrenzengeometrie detaillierter zu beschreiben. Dies beruht im wesentlichen auf der Tatsache, dass mit zunehmender Unregelmäßigkeit der Korngrenzengeometrie, die Länge bzw. dreidimensional betrachtet die Fläche der Korngrenze zunimmt und daraus auch eine stärkere Bindung der Kristalle untereinander resultieren sollte. Die Bennennung der Korngrenzengeometrien im mikroskopischen Skalenbereich folgt in dieser Arbeit dem in Abb. 2.4 angegebenen Schema.

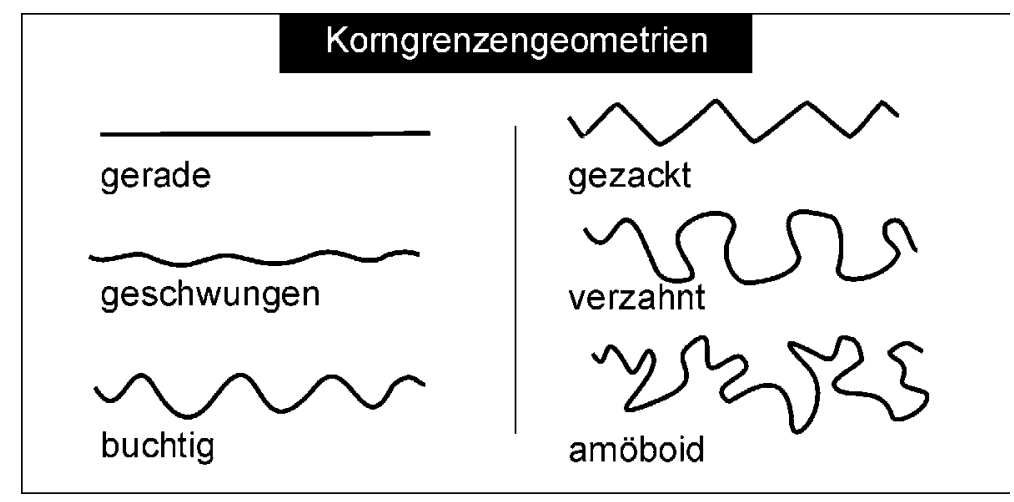

Abb. 2.4: Mikroskopisch erkennbare Korngrenzengeometrien in Marmoren mit zunehmender Kornverbindung und Korngrenzenlänge (vgl. auch WIMMENHAUER, 1985). 


\section{- Kornformregelung}

Formregelungen beschreiben eine anisotrope räumliche Verteilung von Kornlang- oder Kornkurzachsen formanisometrischer Kristalle bzw. Körner. Resultierend ergibt sich aus den Kornformregelung der Kristalle auch eine Vorzugsorientierung der Korngrenzen. Die Bedeutung von Formregelungen für das Materialverhalten ergibt sich in erster Linie daraus, dass die Kristallgrenzen mechanische und strukturelle Diskontinuitäten darstellen und dadurch bevorzugte Richtungen von Bruchmustern und vernetzten Kanälen für Stofftransporte (richtungsabhängige Permeabilitäten) vorzeichnen. Die Entstehung von Formregelungen können auf unterschiedliche geologische Prozesse, wie gerichtetes Längen/Flächenwachstum und/oder Deformation zurückgeführt werden (vgl. auch BLENKINSOP, 2000).

In Marmoren können Formregelungen sowohl von den Hauptmineralphasen Calcit/Dolomit als auch durch Nebengemengteile aufgebaut werden (z.B. Muskovit, Biotit). Hinsichtlich der Geometrie von Formregelungen können diskusförmige (oblate) Kornformen eine Foliation und ellipsenförmige (prolate) Formen eine Lineation erzeugen (s. Abb. 2.5).

\section{Kornformregelungen}

a)

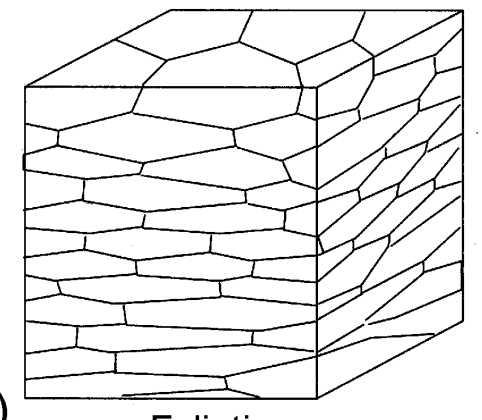

Foliation b)

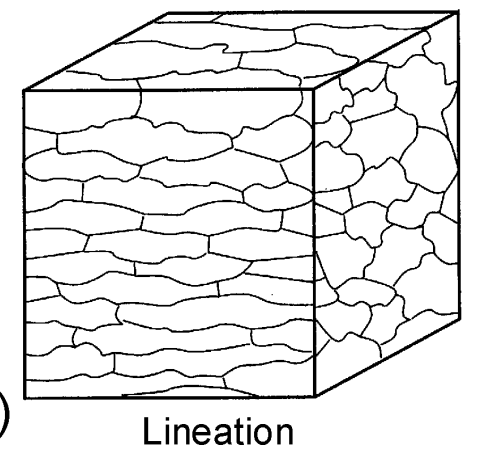

Abb. 2.5: Schematische Darstellung der durch Formregelungen hervorgerufenen a) Foliation und b) Lineation, aus Passchier \& Trouw (1996).

\section{- Textur}

In den Geowissenschaften wird der Begriff Textur traditionell zur Beschreibung der „räumlichen Anordnung und Verteilung von Gemengteilen in einem Gestein“ (MURAwSKI, 1992) angewandt. Diese Verwendung des „Textur“-Begriffs findet sich vor allem in der traditionellen gefügekundlichen Charakterisierung von metamorphen Gesteinen. In diesem Sinne wird der Begriff Textur zur rein beschreibenden, qualitativen Betrachtung verwendet (z.B. Fließtextur, Schlierentextur, etc.). Hingegen wird unter dem Begriff Textur in den Materialwissenschaften vielmehr die „Gesamtheit der Orientierungen der Kristalle eines vielkristallinen Stückes“ verstanden (BUNGE, 1982). In den letzten Jahren hat sich diese Definition, aufgrund der zunehmenden Anwendung metallkundlicher Methoden vor allem in der Strukturgeologie durchgesetzt (vgl. LEISS, 1996). Weitere Informationen insbesondere zu texturbildenden Prozessen s. SKROTZKi (1994). In der vorliegenden Arbeit wird entsprechend der metallkundlichen Definition der Begriff Textur als kristallographische 
Vorzugsorientierung nach dem Kristallgitter (,lattice preferred orientation $=$ LPO“ oder „crystalographic preferred orientation $=\mathrm{CPO}^{\circ}$ ) verwendet.

Für die Textur natürlicher Karbonatgesteine können zwei idealisierte Endglieder betrachtet werden: den c-Achsen und den a-Achsen Fasertyp (s. Abb. 2.6, vgl. LEISS \& UlLEMEYER, 1999). Zwischen diesen beiden Typen sind alle Übergänge zu finden. Fasertexturen sind als Texturen mit axialer Symmetrie definiert und können als Einkristalle, die um eine spezifische Achse rotieren, beschrieben werden. Dabei ist die Achse nicht streng fixiert jedoch zeigt sie ein Maximum um einen zentralen Punkt. Diese Schwächung der Orientierung ist zumeist heterogen und erlaubt damit die Beschreibung bzw. Abgrenzung von natürlich vorkommenden Texturen (Helming, 1996). Im Falle des c-Achsen Fasertyps stellt die cAchse die Rotationsachse (Faser) dar. Dies resultiert in einem einzelnen c-Achsen Punktmaximum auf einem Großkreis und einer a-Achsen Anordnung sowie einer Verteilung aller anderen Flächen, $\mathrm{f}\{012\}, \mathrm{r}\{104\}$ und e $\{018\}$, auf Kleinkreisen um die c-Achse. Für einen a-Achsenfasertyp ist hingegen die a-Achse die Rotationsachse. Hieraus resultiert ein Punktmaximum der a-Achsen und eine mehr oder minder deutliche Ausprägung eines großen Gürtels der c-Achsen. Alle Normalen der weiteren Kristallflächen befinden sich auf einem Kleinkreis um das a-Achsen Punktmaximum.

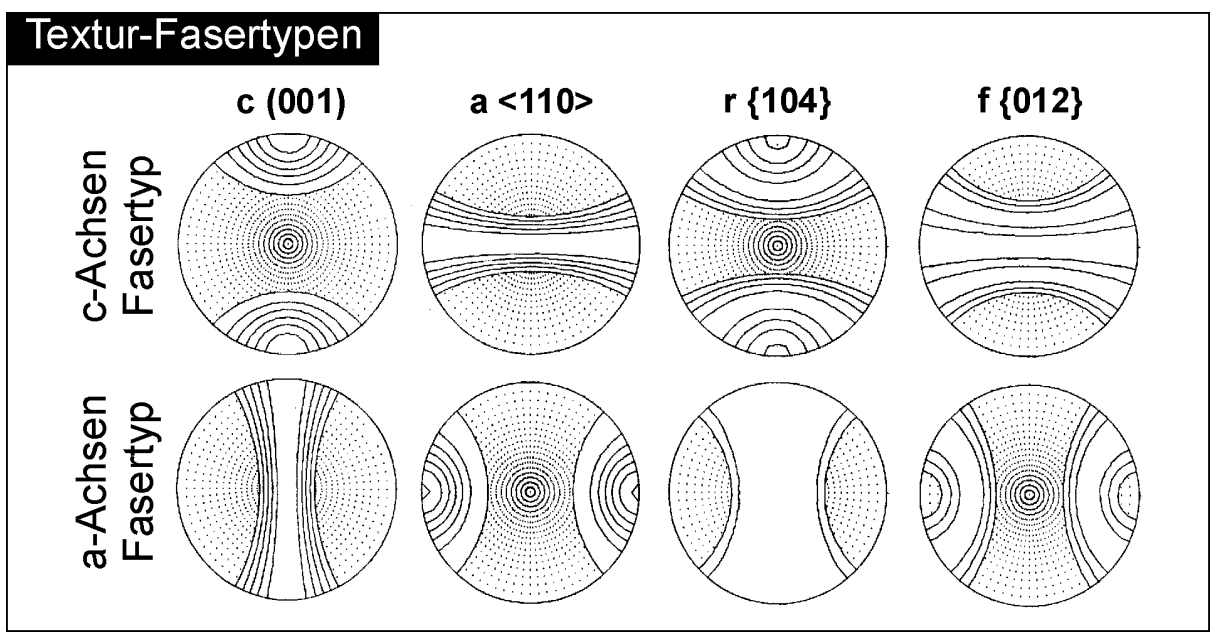

Abb. 2.6: Schematisierter c- und a-Achsen Fasertyp als idealisierte Endglieder der in Marmoren auftretenden Texturtypen, aus LEISS \& ULLEMEYER (1999).

Nicht nur der Typ bzw. das Muster charakterisiert eine Textur sondern auch die Intensität und die Orientierung zu anderen Gefügeelementen wie Foliation und Lineation. Es ist darauf hinzuweisen, dass die definierten Texturtypen nicht notwendigerweise einen texturformenden Mechanismus implizieren.

\section{- Zwillinge}

Zwillinge sind Kristallverwachsungen, wobei die einzelnen Kristallindividuen eine unterschiedliche, jedoch genau festliegende Orientierung besitzen. Für Calcit, insbesondere in Marmoren, sind am weitesten verbreitet sog. Berührungszwillinge nach dem negativen Rhomboeder (112). Sehr häufig kommt eine lamellare Wiederholung in Form von polysynthetischen Zwillingen vor, welche als feine Parallelstreifung auf Spaltflächen 
erkennbar ist. Die polysynthetische Zwillingslamellierung wird häufig durch Deformationsprozesse hervorgerufen. Diese Druckzwillinge weisen dann vielfach auch eine deformationsbedingte Verbiegung auf (vgl. Abb. 2.7; PASSCHIER \& TROUW, 1996).
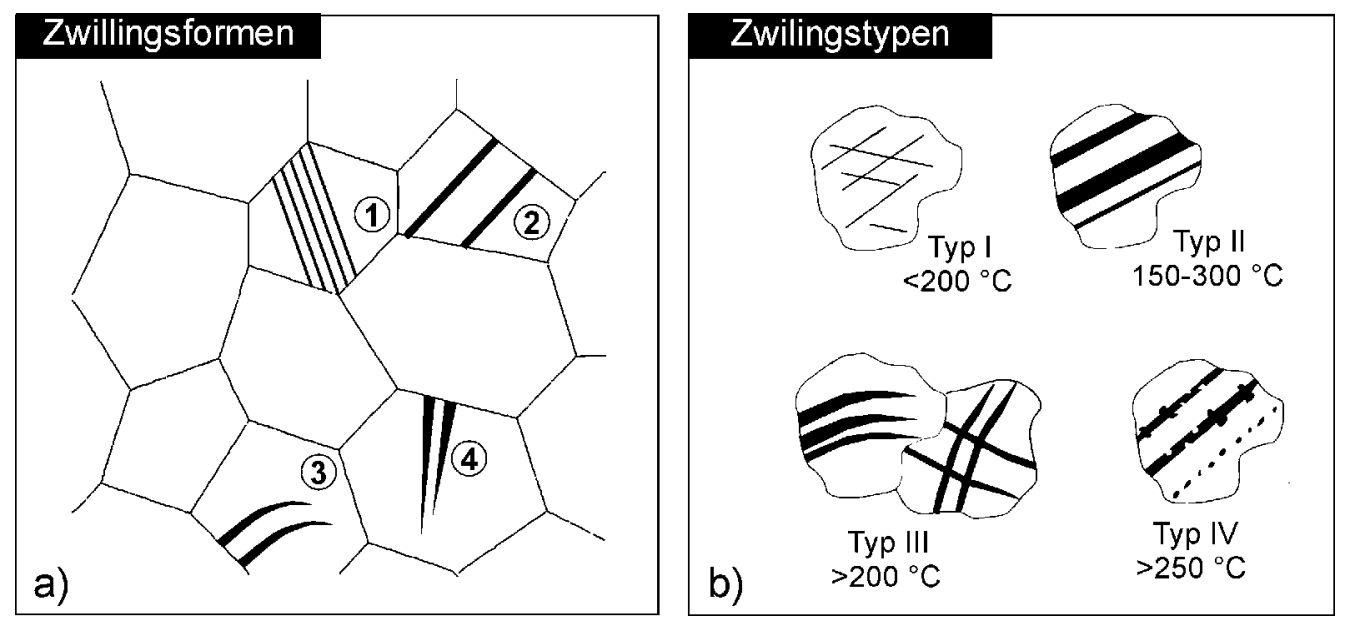

Abb. 2.7: a) Verschiedene Erscheinungsformen von Zwillingen in Calcit-Kristallen $(1=$ engständige Lamellen, 2 = weitständig, 3 = verbogen, 4 = verjüngend auslaufend) und b) Zwillingstypen und ihre Entstehungstemperaturen, aus PASSCHIER \& TROUW (1996).

Die Bedeutung von Zwillingen für die mechanischen und physikalischen Eigenschaften eines Marmors beruhen vor allem darauf, dass sie Diskontinuitäten im Gestein darstellen, die durch eine Beanspruchung bevorzugt aktiviert werden können. Besonders die durch eine Textur hervorgerufene bevorzugte Orientierung von Zwillingen kann zu einer starken Schwächung des Gesteins in distinkten Richtungen führen.

\section{- Mikrorisse}

Mikrorisse sind mikroskopische Bruchstrukturen, welche offen oder in verschiedenster Art mit Mineralen gefüllt sein können. Bei letzteren kann zwischen mineralisierten bzw. versiegelten und verheilten Rissen unterschieden werden (s. Abb. 2.8a, vgl. ATKINSON, 1987). Mikrorisse folgen i.d.R. dem Verlauf gesteinsinterner Schwächezonen. Dabei kommen sie als intergranulare Risse (Korngrenzenrisse), als intragranulare Risse (an Spaltflächen und Zwillingsebenen) und als transgranulare Risse (das Gestein durchziehend) vor. Letztere folgen dabei zumeist wiederum den Korngrenzen wie auch den kristallinternen Diskontinuitäten (s. Abb. 2.8b). Zur weiteren Terminologie von Mikrorissen siehe auch KRANZ (1983).

Offene Mikrorisse sind die in kristallinen Marmoren am häufigsten vorkommenden Risstypen und repräsentieren ihre Porosität. Sie werden sowohl in der geologischen Entwicklung, während der Gewinnung und Verarbeitung sowie vor allem während der Verwitterung des Gesteins angelegt. Offene Mikrorisse besitzen dabei einen großen Einfluss auf das mechanisch physikalische Verhalten von Marmoren. Sie stellen eine wesentliche Schwächung des Gefüges dar.

Mineralisierte und versiegelte Mikrorisse beschreiben mit Mineralen ausgefüllte Bruchstrukturen. Sie sind vor allem durch eine scharfe Begrenzung der Minerale zu den 
Risswandungen und durch zumeist abweichende Körnung zum umgebenden Gestein gekennzeichnet. Diese Risse lassen sich schon mittels herkömmlicher Methoden wie der Polarisationsmikroskopie erfassen. Die versiegelten Risse unterscheiden sich durch die Beteiligung verschiedener Mineralphasen an der Ausfüllung der Bruchstruktur von den mineralisierten Rissen, welche die gleichen Mineralphasen wie das umgebende Gestein aufweisen.

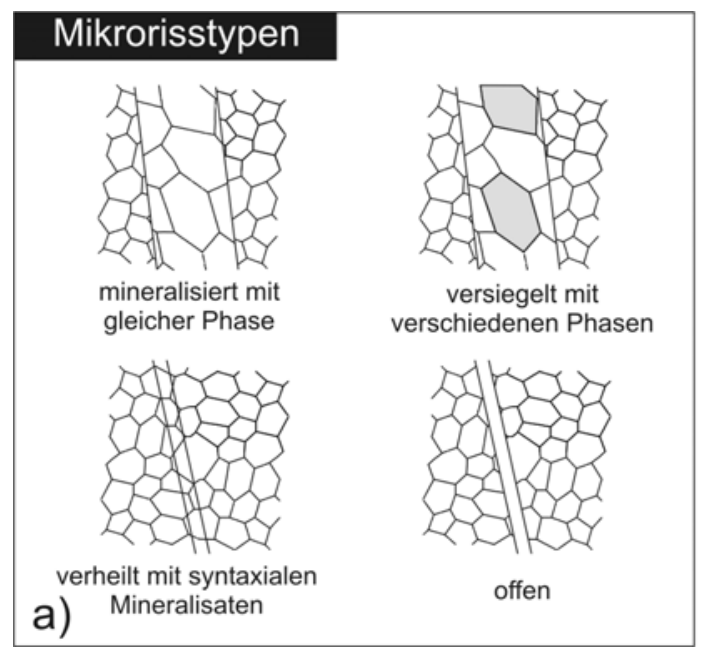

Abb. 2.8: Schematische Darstellung von in verschiedene Mikrorisstypen und 2,6 = intergranular, 3-5 = intragranular).

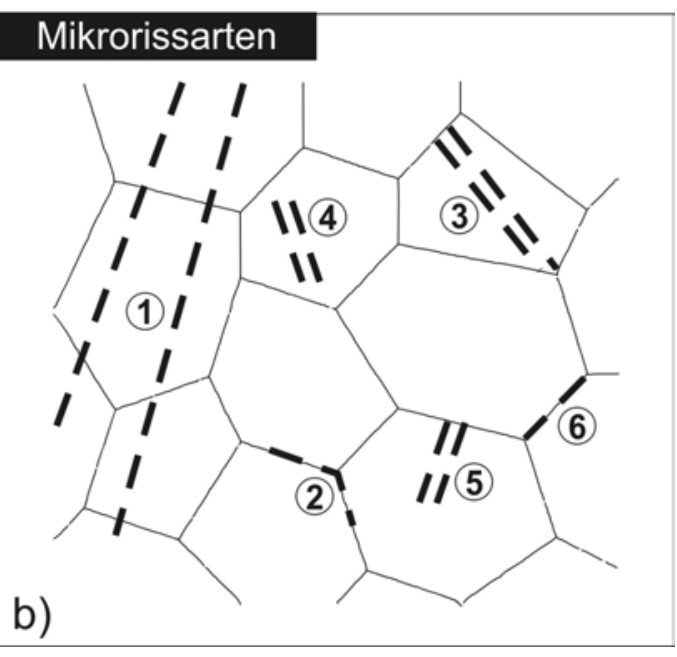

Marmoren auftretenden Mikrorissen: a) b) Mikrorissarten $\quad(1=$ transgranular,

Die verheilten Mikrorisse sind hingegen generell mit der gleichen Mineralphase wie das umgebende Gestein gefüllt. Ihr wesentliches Merkmal ist das Anwachsen der Mineralisate an die angrenzenden Minerale mit gleicher optischer Orientierung (syntaxiale Anwachsung). Damit ist in vielen Fällen eine Erfassung dieser Risse nur bedingt möglich. Häufig sind die verheilten Risse durch Flüssigkeits- und Mineraleinschlüsse, die girlandenartig im Dünnschliff auftreten, nachgezeichnet. Eine Möglichkeit der Erfassung dieser Risse ist die Kathodolumineszenz-Mikroskopie, da die Mineralisate gegenüber dem Muttergestein i.d.R. veränderte chemische Zusammensetzung aufweisen (vgl. MARSHALL, 1988, GROSS et al., 1999). 


\section{Methodik}

\subsection{Grundkonzept und Vorgehensweise}

Das Grundkonzept der vorliegenden Arbeit basiert auf dem Vergleich von Marmoren mit spezifischen Gefügeelementen in fünf verschiedenen Gesteinszuständen: I) bruchfrisch, II) natürlich verwittert, III) künstlich verwittert, IV) modellhaft konserviert und V) konserviert und künstlich verwittert (s. Abb. 3.1). Als Ausgangsmaterial dienten sechs verschiedene Marmortypen (s. Kap. 4.1) im unverwitterten (,bruchfrischen“) und natürlich verwitterten Zustand. Voraussetzung für die Interpretation der Gefügeabhängigkeit von Verwitterungsprozessen bildet eine detaillierte Beschreibung aller relevanten Gefügeelemente des Ausgangsmaterials (s. Kap. 4.2).

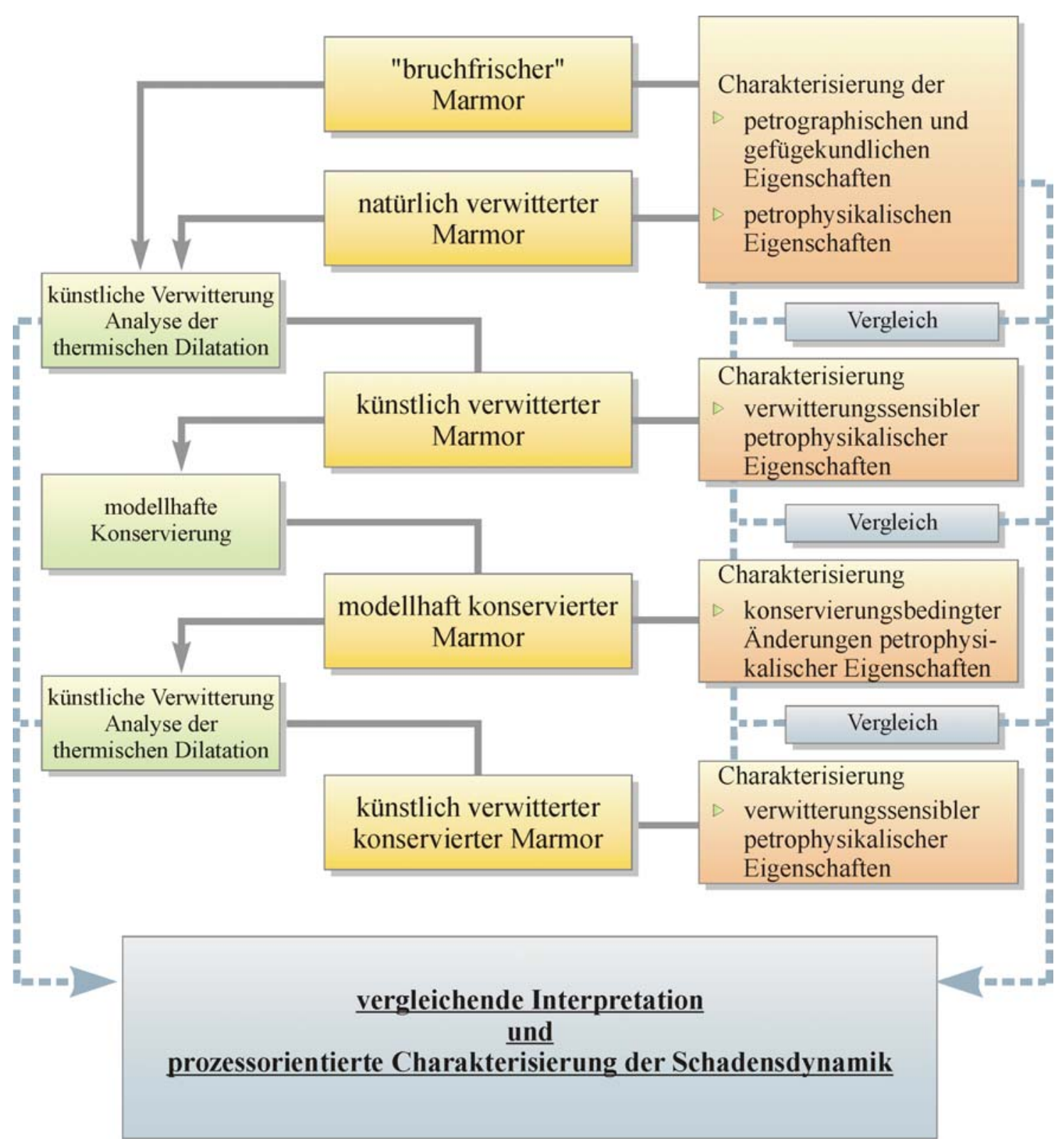

Abb. 3.1: Grundkonzept der vorliegenden Arbeit. 
Die Charakterisierung der verschiedenen Gesteinszustände erfolgte über verwitterungssensible petrophysikalische Parameter (Porosität, Porengrößenverteilung, kapillare Wasseraufnahme, Ultraschallgeschwindigkeiten und Spaltzugfestigkeiten; s. Kap. 4.3). Die künstliche Verwitterung erfolgte thermisch und wurde experimentell mittels Messungen der thermischen Dilatation beschrieben. Thermisch geschädigt wurden die unverwitterten, die natürlich verwitterten und die modellhaft konservierten Marmore. Die Beanspruchung erfolgte durch verschiedene Temperaturstufen, $45^{\circ} \mathrm{C}, 65^{\circ} \mathrm{C}, 90{ }^{\circ} \mathrm{C}$ und $200{ }^{\circ} \mathrm{C}$. Für die Konservierung wurden drei verschiedene Konservierungsverfahren angewandt: I) PMMA auf Lösungsmittelbasis $\left(\mathrm{PMMA}_{\mathrm{sol}}\right)$, II) vorkondensierter Polykieselsäureester (PKSE) und III) Acrylharzvolltränkung $\left(\mathrm{PMMA}_{\text {poly }}\right)$. Eine Überführung der im Labor gewonnen Ergebnisse an die Vor-Ort-Problematik wurde anhand von Fallbeispielen erarbeitet.

\subsection{Probenorientierung und Referenzsystem}

Eine grundlegende Voraussetzung für die Ermittlung von räumlich orientierten Gefügeelementen und ihrer Korrelation mit richtungsabhängigen petrophysikalischen Eigenschaften, ist die Anwendung eines geeigneten Probenreferenzsystems. Hierzu wurde in der vorliegenden Arbeit eine universale Orientierung gewählt (X, Y, Z) und auf die makroskopisch sichtbaren Elemente der Foliation und Lineation ausgerichtet (s. Abb. 3.2). Bei einigen der ausgewählten Marmorproben konnte keine eindeutig interpretierbare Foliation oder Lineation festgestellt werden. In diesen Fällen wurde nach den vorgegebenen Flächen der Bauteilgeometrie das Referenz-Koordinatensystem ausgerichtet.
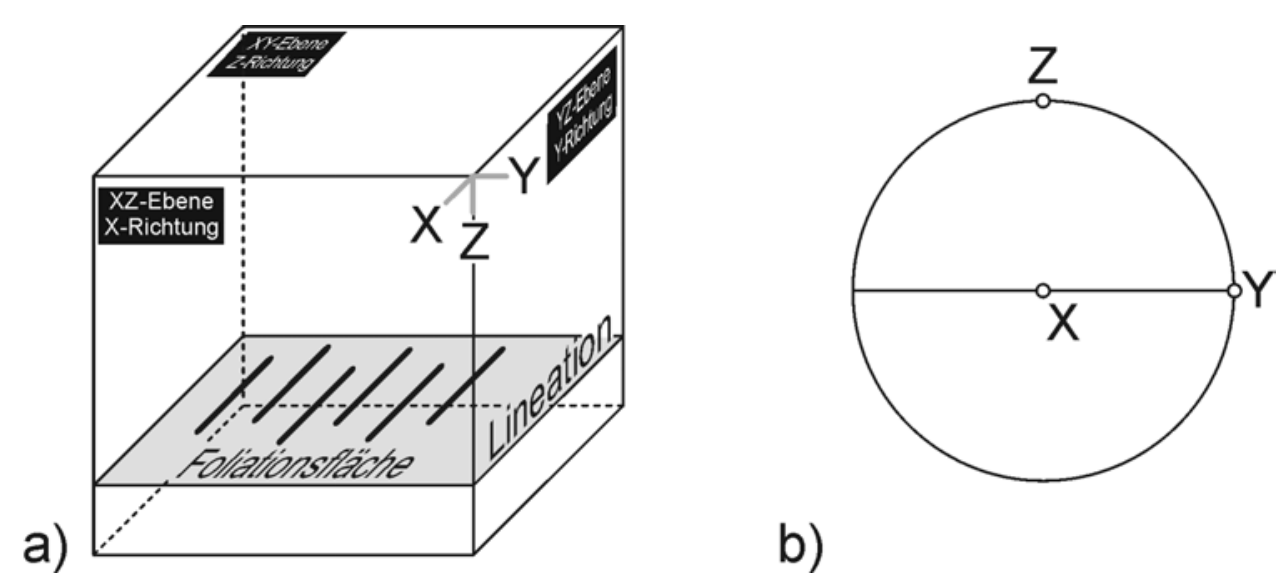

Abb. 3.2: a) Referenz-Koordinatensystem in Bezug auf die makroskopischen Elemente der Foliation und Lineation und b) Darstellung der Hauptrichtungen in einer flächentreuen Projektion.

\subsection{Petrographische und gefügekundliche Analysen}

Grundlegende Voraussetzung für die Interpretation von Gesteinsverwitterungsprozessen ist die fundierte Zustandsbeschreibung der petrographischen und gefügekundlichen Kenngrößen des Naturwerksteins. Hierzu stellen herkömmliche mikroskopische Analyseverfahren immer noch unverzichtbare Hilfsmittel dar, deren Vorteil in der einfachen Durchführbarkeit liegt. Zur quantitativen Erfassung der Kornparameter wurden bildanalytische Verfahren eingesetzt. 
Mittels Neutronentextur erfolgte die Analyse der kristallographischen Vorzugsorientierung. Die mikroskopischen und submikroskopischen Untersuchungen wurden an polierten Standarddünnschliffen, mit einer Raumlage entsprechend dem Koordinatensystem parallel zur XY-, XZ- und YZ-Ebene, durchgeführt.

\section{- Mikroskopische Analysen}

Die konventionelle Polarisationsmikroskopie (POL) wurde zur Erfassung des Mineralbestandes und der mikrostrukturellen Charakteristik eingesetzt. Vor allem ermöglicht dieses Analyseverfahren eine einfache und damit schnelle, qualitative Beschreibung des Gefügeinventars. Hinsichtlich der Entfestigungsprozesse erlaubt die Polarisationsmikroskopie die Aufnahme potentieller Schwachstellen des Gefüges, wie z.B. Rissmineralisationen, evtl. Lösungssuturen oder Deformationsstrukturen, die bei mechanischer Beanspruchung aktiviert werden können oder Wegsamkeiten für fluide Phasen darstellen.

Die optische Kathodolumineszenz-Mikroskopie (KLM) wurde zur qualitativen Beschreibung von Besonderheiten des Gefügeinventars und von Fluid-Gesteinswechselwirkungen eingesetzt. Die KLM ermöglicht in den meisten Fällen eine einfache Unterscheidbarkeit der beiden in kristallinen Marmoren vorkommenden Hauptmineralphasen Calcit und Dolomit. Für einen methodischen Überblick und zur Anwendung dieses Verfahrens hinsichtlich geowissenschaftlicher Fragestellungen siehe z.B. MARSHALL (1988) und NEUSER (1988).

Die Rasterelektronenmikroskopie (REM) diente der hochauflösenden Visualisierung von Art, Ausbreitung und Verteilung der Bruchstrukturen in den untersuchten Marmoren. Von entscheidender Bedeutung ist dabei, inwieweit Unterschiede in der Korngrenzenmorphologie bruchfrischer und verwitterter Proben existieren oder ob Korrosionsphänomene bereits neben der reinen mechanischen Gefügeauflockerung Stoffumlagerungen erkennen lassen. Ferner wurde diese Methode zum Nachweis des Vorkommens und der Verteilung von eingebrachten Steinfestigern in den Porenraum von Marmoren angewandt. Die Untersuchungen wurden an Bruchproben (Fraktographie) durchgeführt.

Die Fluoreszenz-Mikroskopie (FLM) wurde zur Visualisierung von Rissmustern und zur Erfassung der jeweiligen Rissarten (intergranular, intragranular usw.) verwendet. Dazu wurde ein Epoxidharz (mit Fluoreszenzmittel versetzt) unter Vakuum-/Druckstufen in die Probenstücke eingebracht.

\section{- Bildanalytische Gefügeauswertung}

Mittels Bildanalyse wurde eine Quantifizierung der wesentlichen Parameter des Korngefüges durchgeführt (Kornformregelung, Korngrenzenkonfiguration, Korngrößenverteilung, s. Abb. 3.3). Aus den Formregelungen können bereits Abschätzungen des Materialverhaltens abgegeben werden, da die Kristallgrenzen mechanische und strukturelle Diskontinuitäten darstellen. Die Untersuchungen wurden an drei senkrecht zueinander liegenden Dünnschliffen, entsprechend dem Koordinatensystem parallel zur XY-, XZ- und YZ-Ebene, durchgeführt. 

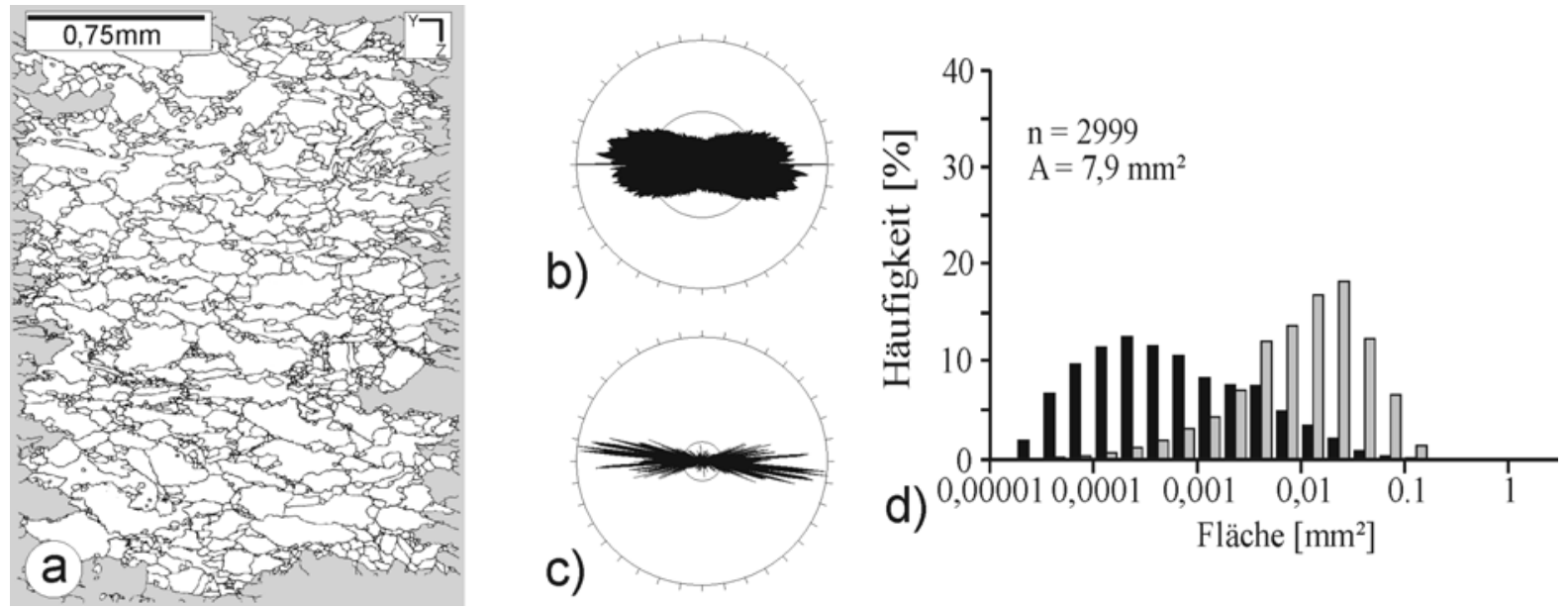

Abb. 3.3: Darstellung der mittels Bildanalyse ermittelten Gefügeparameter: a) Korngefüge eines repräsentativen Dünnschliffausschnittes als Eingabe für die digitale Auswertung, b) bevorzugte Regelung der Korngrenzen, c) bevorzugte Regelung der Langachsen und d) Korngrößenverteilung als Kornflächenparameter dargestellt ( $=$ Kornanzahl, $\mathrm{A}=$ Gesamtfläche).

Aufgrund des häufigen Auftretens von Zwillingsbildung und Spaltflächen ist bei Marmoren eine vollautomatische Bildanalyse mit herkömmlichen Techniken nicht möglich, da die existierenden Programme nicht zwischen Korngrenze und Zwillings- oder Spaltflächengrenze unterscheiden können. Darauf beruhend erfolgte in dieser Arbeit eine „halbautomatische Bildanalyse“ (vgl. POSCHLOD, 1989). Dazu wurden die einzelnen Körner nachgezeichnet, digitalisiert und im folgenden mit Hilfe des Analyseprogramms DIANA quantitativ ausgewertet. Für weitere Details dieses Verfahrens und des verwendeten Auswerteprogramms s. DUYSTER (1991).

\section{- Neutronentextur}

Die Texturanalyse wurde zur Erfassung des Musters und der Intensität der kristallographischen Vorzugsregelung der Calcit-Kristalle angewandt. Die Notwendigkeit einer quantitative Bestimmung ergibt sich aus dem Einfluss der Textur auf die petrophysikalischen Eigenschaften (z.B. Richtungsabhängigkeit der thermischen Dilatation). Für die Bestimmung der Textur der verschiedenen Marmortypen wurde die Neutronendiffraktionsmethode eingesetzt. Gegenüber der herkömmlichen Röntgentextur-Goniometrie ermöglicht diese Technik, auch die Messung grob kristalliner Gesteine mit einer Korngröße über $100 \mu \mathrm{m}$ (s. LEISS \& UlLEMEYER, 1999). Dies ergibt sich aus der größeren Eindringtiefe der Neutronen in den Probekörper. Zur Verwendung kamen zylindrische Proben (30 mm x $30 \mathrm{~mm}$ ), die auch bei durchschnittlichen Korngrößen von 1,5 mm eine ausreichende Statistik gewährleisten. Die Untersuchungen wurden am Neutronen Diffraktometer (SKAT) des „Pulse Reactor“ IBR - 2 in Dubna (Russland) durchgeführt.

Ausgewertet wurden die Basisfläche (006), die a-Achse $<110>$, die Spaltrhomboederfläche $\{104\}$, die Zwillingsflächen $\{012\}$ und $\{018\}$. Für die Darstellung der quantitativen Texturdaten wurden Polfiguren errechnet und in eine Schmidt'sche Netz Projektion überführt (vgl. Abb. 3.4). 


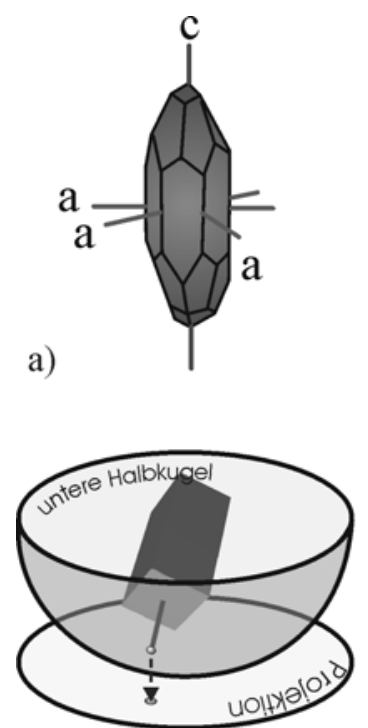

b)
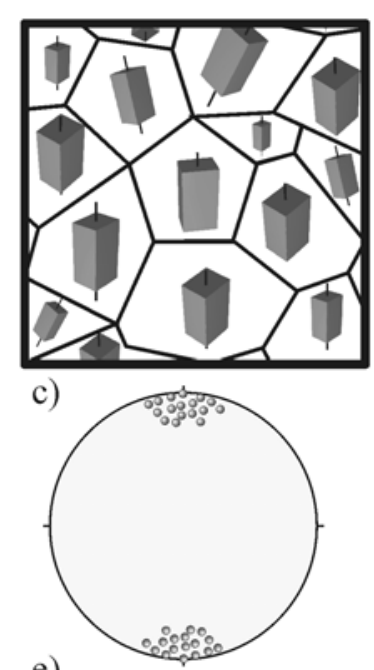

e)

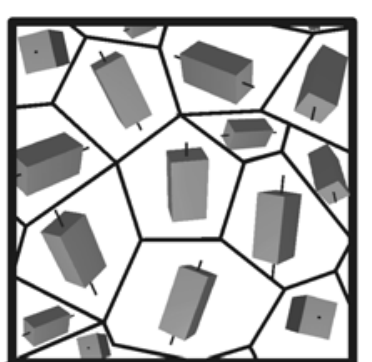

d)

f)

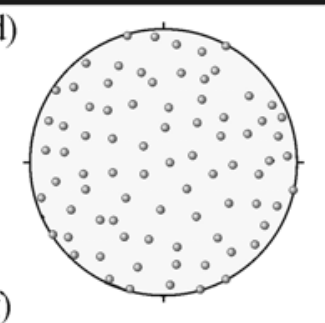

Abb. 3.4: Schematische Darstellung einer Textur und die Überführung in eine Projektion (Schmidt'sches Netz, untere Halbkugel): a) Calcit-Einkristall und b) Projektion der c-Achse eines Kristalls mit schräger Raumlage, c) geregelte Kristalle und e) ihre Projektion, isotrop verteilte Kristalle und f) ihre Projektion, aus SIEGESMUND et al. (2001).

\subsection{Petrophysikalische Analysen}

\subsubsection{Porosität und Porengrößenverteilung}

Der Porenraum besitzt für das Verwitterungsverhalten von Naturwerksteinen entscheidende Bedeutung, da die mechanischen und physikalischen Eigenschaften (z.B. Wassertransportmechanismen) eines Gesteins durch die Art, Form, Größe, Verteilung und Vernetzung der Poren kontrolliert werden. Die Porosität stellt das Verhältnis zwischen dem Hohlraumvolumen (Poren) und dem Gesamtvolumen des Gesteins dar und wird in Prozent angegeben. In der vorliegenden Arbeit wurde die effektive Porosität (zugänglicher Porenraum) ermittelt.

Die Bestimmung der Porosität erfolgte über die verschiedenen Gewichtszustände, Trockengewicht $\left(\mathrm{m}_{\mathrm{t}}\right)$, Nassgewicht $\left(\mathrm{m}_{\mathrm{n}}\right)$ sowie Auftriebsgewicht $\left(\mathrm{m}_{\mathrm{a}}\right)$, wobei als porensättigendes Medium demineralisiertes Wasser verwendet wurde. Die Sättigung des zugänglichen Porenraumes wurde durch vorhergehendes Evakuieren der Proben erreicht. Die Messungen erfolgten an Würfelproben mit einer Kantenlänge von $65 \mathrm{~mm}$. Die Porosität ergibt sich aus der Beziehung (Formel 3.1):

Formel 3.1:

$$
\begin{array}{llll}
\Phi & {[\%]} & =\text { Porosität } \\
\Phi=\frac{m_{n}-m_{t}}{m_{n}-m_{a u}} \cdot 100 & \mathrm{~m}_{\mathrm{t}} & {[\mathrm{g}]} & =\text { Trockengewicht } \\
& \mathrm{m}_{\mathrm{n}} & {[\mathrm{g}]} & =\text { Nassgewicht } \\
\mathrm{m}_{\mathrm{au}} & {[\mathrm{g}]} & =\text { Auftriebsgewicht }
\end{array}
$$

Aus den Gewichtsdaten lassen sich weiterhin die Rohdichte $\left(\rho_{\text {roh }}\right)$ und die Matrixdichte ( $\left.\rho_{\text {mat }}\right)$ bestimmen (Formel 3.2 und 3.3). 
Formel 3.2:

$\rho_{\text {roh }}=\frac{m_{t}}{m_{n}-m_{a u}} \quad \quad \rho_{\text {roh }} \quad\left[\mathrm{g} / \mathrm{cm}^{3}\right]=$ Rohdichte

Formel 3.3:

$$
\rho_{m a t}=\frac{m_{t}}{m_{t}-m_{a u}}
$$

$\rho_{\text {mat }}$

$\left[\mathrm{g} / \mathrm{cm}^{3}\right]=$ Matrixdichte

Die Ermittlung der Matrixdichte kann bei Marmoren erste Hinweise auf die mineralogische Zusammensetzung geben, da Calcit und Dolomit Marmore anhand ihrer Dichte (vgl. Tab. 2.1) unterscheidbar sind. Zum anderen ermöglicht die Matrixdichte eine Einschätzung der Qualität der Messung, was insbesondere bei sehr gering porösen Gesteinen wie kristallinem Marmor sinnvoll ist.

Die Bestimmung der Porengrößenverteilung erfolgte mittels Quecksilberporosimetrie (vgl. BRAKEL et al., 1981) an zylindrischen Proben $(\varnothing 12,5 \mathrm{~mm} / 40 \mathrm{~mm})$. Die Untersuchungen wurden an zwei vergleichbaren Hg-Porosimetern 2000 der Fa. Carlo Erba am GFZ-Potsdam und am Geographischen Institut der Universität Göttingen durchgeführt. Die Kapillardruckkurven wurden bis zu einem Druck von 2000 bar gemessen, was die Erfassung bis zu einem Porenradius von 3,8 nm ermöglicht. Dem Messverfahren liegt die WashburnGleichung zugrunde (Formel 3.4). Danach gilt für nicht benetzende Flüssigkeiten mit einem Randwinkel $\theta>90^{\circ}$, dass jedem Kapillarradius ein bestimmter Druck zugeordnet werden kann, der aufgebracht werden muss um die Flüssigkeit in die Kapillare einzupressen.

Formel 3.4:

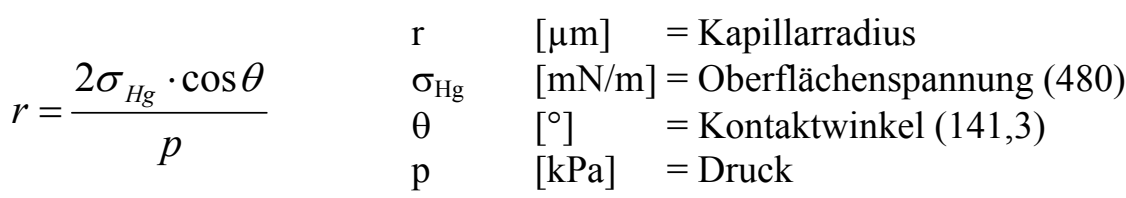

Gemessen wird der kreisäquivalente Radius einer idealisierten zylindrischen Pore. Für komplexere Poren wird der kleinste Querschnitt, also bei Mikrorissen die Rissweite, bestimmt. Es kann davon ausgegangen werden, dass die Rissporen von kristallinen Gesteinen nur näherungsweise beschrieben werden können (vgl. POSCHLOD, 1989).

Die Verteilung der Porengrößen gibt den Anteil unterschiedlicher Porengrößen (Porenklassen) am Porenvolumen eines Gesteins wieder. Für Marmor ist die Porengrößenverteilung sehr stark vom Erhaltungszustand abhängig und repräsentiert damit einen verwitterungssensiblen petrophysikalischen Parameter (vgl. RUEDRICH et al., 2001a). In der vorliegenden Arbeit wurde die Messung der Porengrößenverteilungen eingesetzt, um die zunehmende Entfestigung $\mathrm{zu}$ charakterisieren. Dazu wurden die Porengrößen verschiedener Gesteinszustände (natürlich verwittert, thermisch beansprucht und modellhaft konserviert) ermittelt und miteinander verglichen. Bezüglich der modellhaften Konservierungen dienten die Messungen, die Besetzung der jeweiligen Porenklassen durch die Imprägnierungsmittel zu detektieren. 


\subsubsection{Kapillare Wasseraufnahme}

Die richtungsabhängige kapillare Wasseraufnahme wurde zur Ermittlung der Vernetzung des Porenraums und der Intensität des kapillaren Wassertransports durchgeführt. Letzteres besitzt aufgrund der hohen Löslichkeit des Calcit-Kristalls bei der Verwitterung von Marmoren eine große Bedeutung.

Die Messung der kapillaren Wasseraufnahme wurde mit einer Unterflur-Wägeeinrichtung durchgeführt. Als Untersuchungsmaterial kamen Gesteinswürfel (Kantenlänge $=65 \mathrm{~mm}$ ) zur Verwendung, die sich mit ca. $10 \mathrm{~mm}$ des unteren Probenteils in einem Wasserbasin befanden. Die Richtungsabhängigkeit wurde durch drehen der Probe in die drei orthogonalen Gefügerichtungen ermittelt. Mit dieser Vorgehensweise konnte die Richtungsabhängigkeit an einem Würfel durchgeführt und damit der Einfluss von Materialheterogenitäten weitgehend minimiert werden. Die Datenaufnahme erfolgte digital alle 20 Sekunden.

Aus den Daten der Wasseraufnahme können verschiedene Kennwerte ermittelt werden, mit denen das Gesteinsverhalten beschrieben und verglichen werden kann. Dazu wurden in der vorliegenden Arbeit der Wasseraufnahmekoeffizient (w-Wert) und der Sättigungsgrad der Gesteine ermittelt.

Der w-Wert gibt Information darüber, wie viel Wasser über eine bestimmte Fläche durch kapillare Saugkraft vom Gestein aufgenommen werden kann (s. Formel 3.5; vgl. PoschLOD, 1989).

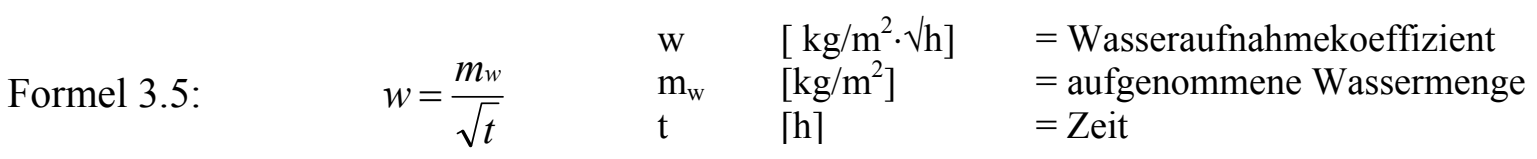

Ein weiterer wichtiger Parameter zur Charakterisierung des Wasseraufnahmevermögens von Gesteinen ist der Sättigungsgrad (s. Formel 3.6). Dabei handelt es sich um das Verhältnis der maximalen Wasseraufnahme (zugänglicher Porenraum unter Vakuum) und der freiwilligen Wasseraufnahme unter Normalbedingungen (Atmosphärendruck) eines Gesteins. Für letzteren Wert wurden in dieser Arbeit die Daten der beschriebenen kapillaren Wasseraufnahme herangezogen.

\begin{tabular}{|c|c|c|c|c|}
\hline & & $\mathrm{S}$ & & $=$ Sättigungsgrad \\
\hline Formel 3.6: & $S=\frac{W_{v a k}}{W_{a t m}}$ & $\begin{array}{l}\mathrm{W}_{\text {vak }} \\
\mathrm{W}_{\text {atm }}\end{array}$ & $\begin{array}{l}{[\mathrm{g}]} \\
{[\mathrm{g}]}\end{array}$ & $\begin{aligned}= & \text { aufgenommene Wassermenge Vakuum } \\
= & \text { aufgenommene Wassermenge unter } \\
& \text { Atmosphärendruck }\end{aligned}$ \\
\hline
\end{tabular}

\subsubsection{Thermische Längenänderung}

Das thermische Expansionsverhalten der untersuchten Marmore wurde an Gesteinszylindern (50 mm / $\varnothing 10 \mathrm{~mm}$ ) ermittelt, welche parallel zu den orthogonalen Gefügerichtungen (X, Y, Z) entnommen wurden. Die Richtungsabhängigkeit der thermischen Dilatation wurde dabei als Funktion der Temperatur ermittelt. Die Untersuchungen erfolgten an einem Dilatometer der Technischen Universität Wien mit induktiven Wegaufnehmern. Die Messeinrichtung 
besitzt eine Auflösung von $1 \mu \mathrm{m}$. Ein besonderer Vorteil der verwendeten Messapparatur ist die Möglichkeit der Parallelmessung von drei Proben. Als geeignete Heizrate hat sich eine Temperaturerhöhung von $0.75{ }^{\circ} \mathrm{C} \mathrm{min}^{-1}$ herausgestellt. Als Referenzmaterial wurde Quarzglas verwendet, welches einen besonders niedrigen, isotropen thermischen Ausdehnungskoeffizienten von $\alpha=0.5 * 10^{-6} \mathrm{~K}^{-1}$ aufweist. Für weitere Details der Messeinrichtung s. WiDHALM et al. (1996).

Die Darstellung von Dilatationsdaten erfolgt gewöhnlich durch die Berechnung eines Ausdehnungskoeffizienten, der nach Formel 3.7 ermittelt wird.

\begin{tabular}{|c|c|c|c|c|}
\hline Formel 3.7: & $\alpha=\frac{\Delta l}{l \cdot \Delta T}$ & $\begin{array}{l}\alpha \\
\Delta 1 \\
\Delta \mathrm{T} \\
1\end{array}$ & $\begin{array}{l}{\left[\mathrm{K}^{-1}\right]} \\
{[\mathrm{mm}]} \\
{\left[\mathrm{K}^{-1}\right]} \\
{[\mathrm{mm}]}\end{array}$ & $\begin{array}{l}=\text { Ausdehnungskoeffizient } \\
=\text { Längenänderung } \\
=\text { Temperaturänderung } \\
=\text { Probenlänge }\end{array}$ \\
\hline
\end{tabular}

Zur besseren Veranschaulichung ist der Wert $\varepsilon$ besser geeignet, der die absolute Längenänderung widerspiegelt. Er wird nach Formel 3.8 berechnet, wobei $\Delta \mathrm{l}$ aus Anschauungsgründen in [mm] angegeben wird.

\begin{tabular}{|c|c|c|c|}
\hline Formel 3.8 & $\varepsilon=\frac{\Delta l[\mathrm{~mm}]}{l[\mathrm{~m}]}$ & $\begin{array}{l}\varepsilon \\
\Delta 1 \\
1\end{array}$ & 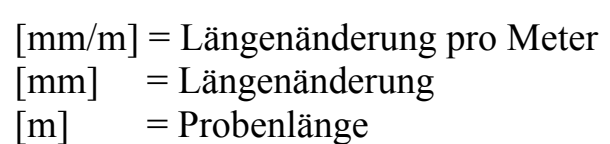 \\
\hline
\end{tabular}

Gängige Werte für $\varepsilon$, ermittelt durch das Aufheizen von Marmorproben bis auf $200{ }^{\circ} \mathrm{C}$, liegen in der Größenordnung von 0.5-1.5 mm/m (WIDHALM et al., 1996). Diese Werte entsprechen einer Längenänderung einer Probe mit $5 \mathrm{~cm}$ Länge in der Größenordnung von 25-75 $\mu \mathrm{m}$, was deutlich über der Nachweisgrenze von $1 \mu \mathrm{m}$ liegt und somit mit der verwendeten Apparatur erfassbar ist. In Abb. 3.5 ist das Längenänderungsverhalten für verschiedene Ausdehnungskoeffizienten, $\alpha=$ groß, $\alpha=$ klein und $\alpha=$ negativ, schematisch dargestellt.

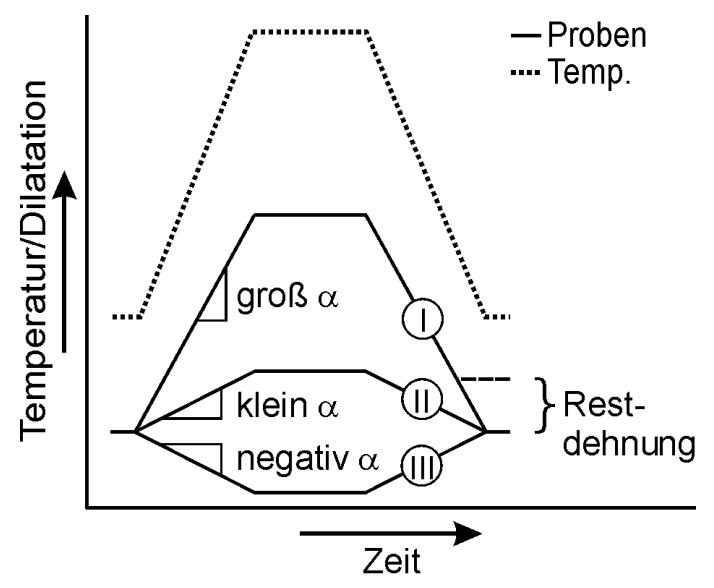

Abb. 3.5: In Experimenten $\mathrm{zu}$ beobachtende Kurven verschiedener thermischer Ausdehnungskoeffizienten $\alpha(\mathrm{I}=$ großer $\alpha, \mathrm{II}=$ kleiner $\alpha$ and III = negativer $\alpha)$. Eine Restdehnung tritt auf wenn die Probe nach der Beanspruchung nicht ihre Ausgangslänge erreicht.

Zur Ermittlung einer möglichen Schädigung in unterschiedlichen Temperaturbereichen, wurden vier Heizrampen eingesetzt. Dazu erfolgte eine zyklische Wärmebehandlung der Proben auf $43{ }^{\circ} \mathrm{C}$, auf $65^{\circ} \mathrm{C}$, auf $90^{\circ} \mathrm{C}$ und in einer wiederholten Rampe auf $90{ }^{\circ} \mathrm{C}$. Letztere 
diente der Ermittlung einer fortschreitenden Schädigung bei wiederholtem Heizen auf die gleiche Temperaturstufe. Zusätzlich wurden Kühlzyklen auf $-25^{\circ} \mathrm{C}$ ausgehend von $20^{\circ} \mathrm{C}$ eingesetzt, um das Längenänderungsverhalten bei Unterkühlung der Proben zu ermitteln.

\subsubsection{Ultraschallanalytik}

Die Ultraschallanalytik diente der Erfassung des Entfestigungszustandes der Marmore (vgl. KÖHLER, 1991; WeISS et al., 2001). Hierzu erfolgte eine vollständige Bestimmung von Kompressionswellengeschwindigkeitsverteilung an Kugelproben und die Ermittlung von Kompressionswellen an Würfelproben in den drei orthogonalen Gefügerichtungen (X,Y und Z).

Die vollständige Bestimmung der $\mathrm{V}_{\mathrm{p}}$ Wellengeschwindigkeitsverteilung wurde an trockenen und wassergesättigten Kugelproben $(\varnothing 50 \mathrm{~mm} \pm 0,02 \mathrm{~mm}$ ) nach der Durchschallungsmethode (pulse transmission technique; BIRCH, 1960, 1961) im Tauchbad ermittelt. Hinsichtlich der trockenen Messungen wurden die Proben mit einem Kunststofffilm gegen das Schallübertragungsmedium Wasser abgedichtet. Der Messaufbau ermöglicht eine Rotation der Kugel um $360^{\circ}$ um die vertikale Achse (s. Abb. 3.6). Die piezokeramischen Tauchschwinger ( $1 \mathrm{MHz}$ Eigenfrequenz) können als Sender- und Empfängerpaar in $10^{\circ}$ Schritten bis $\mathrm{zu} 75^{\circ}$ aus der horizontalen Ebene rotiert werden. Diese Konfiguration ermöglicht eine Laufzeitbestimmung der P-Wellen in 180 unabhängigen Richtungen. Diese vollständige $V_{p}$-Verteilung erlaubt bei geeignetem Probeneinbau das tatsächliche Maximum $\left(\mathrm{V}_{\mathrm{pmax}}\right)$ und das tatsächliche Minimum $\left(\mathrm{V}_{\mathrm{pmin}}\right)$ zu bestimmen (z.B. SIEGESMUND et al., 1993). Eine genauere Beschreibung der Ultraschallgeschwindigkeitsbestimmung an Kugelproben geben WEISS (1998) und JAHNS (1995).
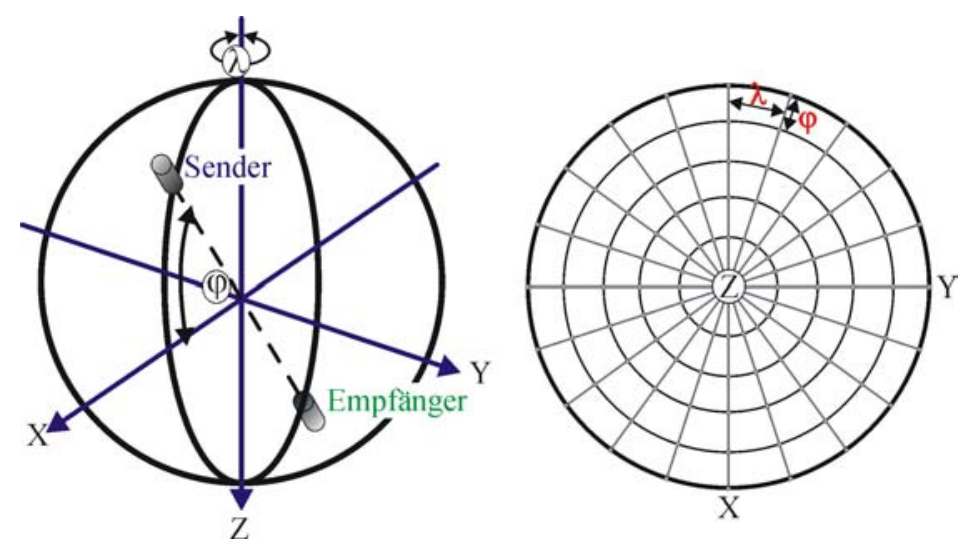

Abb. 3.6: a) Schematische Darstellung des grundlegenden Aufbaus der Kugelmesseinrichtung und b) verwendetes Messraster, aus SIEGESMUND et al. (1993).

Ergänzend zu den Kugelmessungen wurden die Kompressionswellengeschwindigkeiten an trockenen und wassergesättigten Würfelproben (Kantenlänge $=65 \mathrm{~mm}$ ) ermittelt. Als Impulsgeber und Empfänger wurden $1 \mathrm{MHz}$ Kompressionswellenschwinger verwendet. 


\subsubsection{Spaltzugfestigkeit}

Die Ermittlung der Spaltzugfestigkeit $\left(\sigma_{\mathrm{Z}}\right)$ erfolgte mit dem Brazil-Test (indirekte Zugfestigkeit). Dazu wurden liegende zylindrische Prüfkörper (20 mm / $\varnothing 40 \mathrm{~mm}$ ) durch eine Streifenlast längs zweier gegenüberliegender, gerader Mantellinien bis zum Bruch durch Spalten der Probe belastet. Die Zugbeanspruchung tritt dabei rechtwinklig zur Belastungsrichtung auf. Unter der Kraft F bildet sich bei Gültigkeit des linearen Elastizitätsgesetzes für Streifenlasten in der Belastungsebene eine Spannungsverteilung entsprechend des Diagramms der Abb. 3.7a,b aus.

Im Schnitt durch die Probe ist die Vertikalspannung $\sigma_{\mathrm{V}}$ eine Druckspannung, die Horizontalspannung $\sigma_{\mathrm{H}}$ ist über ca. $70 \%$ des Prüfkörperdurchmessers eine gleichmäßig verteilte Zugspannung. Lediglich in den Krafteinleitungszonen ist $\sigma_{\mathrm{H}}$ eine Druckspannung. Die Anwendung des Spaltzugversuchs zur Bestimmung der Zugfestigkeit geht von der experimentell gewonnen Erfahrung aus, dass eine Gesteinsprobe dann zu Bruch geht, wenn die Zugspannung den Wert der Gesteinszugfestigkeit $\sigma_{Z}$ erreicht.
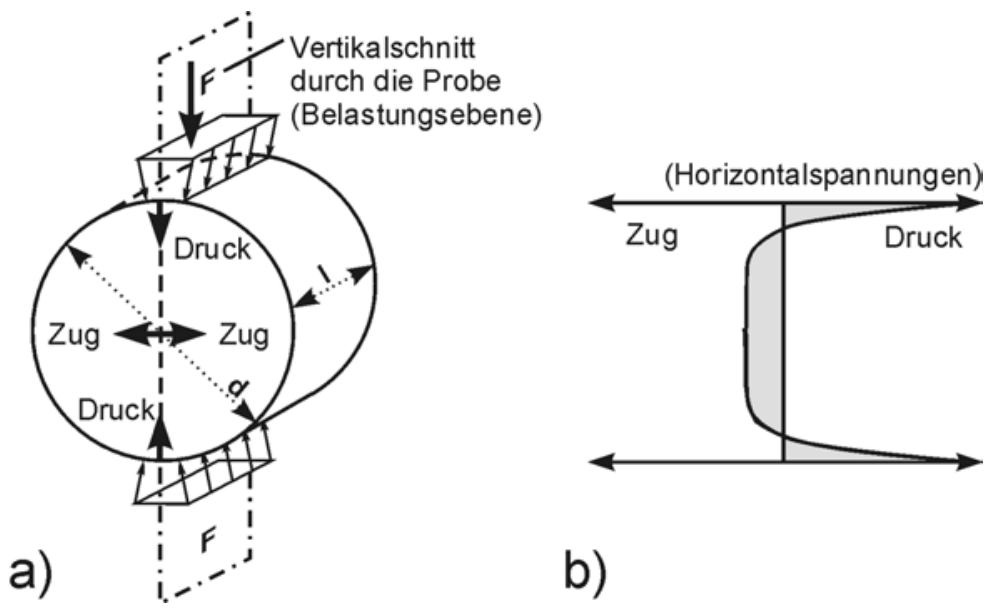

b)

Abb. 3.7: Darstellungen zur Spaltzugfestigkeitsermittlung nach dem Brazil-Test: a) Prinzip der Versuchanordnung und b) schematische Darstellung des Spannungszustandes in einer belasteten Probe.

Die Versuchsdurchführung erfolgte in Anlehnung an DIN 22024. Die Kraft wurde über eine $20 \mathrm{kN}-K$ raftmessdose mit einer Auflösung von $\pm 0,5 \%$ gesteuert und gemessen. Als Belastungsgeschwindigkeit wurde eine Kraftzunahme von $30 \mathrm{~N} / \mathrm{s}$ gewählt, was bei der gewählten Probendimension einer Spannungszunahme in der Probe von $0,024 \mathrm{MPa} / \mathrm{s}$ entspricht. Aus der Bruchlast F und den Prüfkörperabmessungen (s. Abb. 3.7a) wird die Spaltzugfestigkeit mit Formel 3.9 ermittelt.

Formel 3.9:

$$
\sigma_{Z}=\frac{2 \cdot F}{d \cdot l \cdot \pi}
$$

$\begin{array}{lll}\sigma_{\mathrm{Z}} & \text { Bruchspannung } & {[\mathrm{MPa}]} \\ \mathrm{F} & \text { Bruchkraft } & {[\mathrm{kN}]} \\ 1 & \text { Prüfkörperlänge } & {[\mathrm{cm}]} \\ \mathrm{d} & \text { Prüfkörperdurchmesser } & {[\mathrm{cm}]}\end{array}$

Jeder Spaltzugfestigkeitswert wurde aus mindestens vier gemittelten Parallelmessungen gewonnen, wobei die Standardabweichung angegeben ist. 


\section{Untersuchte Marmore}

Die Untersuchungen der vorliegenden Arbeit wurden an sechs verschiedenen Marmortypen durchgeführt. Dabei handelt es sich um die polnischen Marmore aus Prieborn, Kauffung und Grosskunzendorf sowie um die italienischen aus Carrara, Lasa und Sterzing.

Die verwitterten Gesteinsproben des Carrara, Prieborner, Grosskunzendorfer und Kauffunger Marmors repräsentieren Austauschmaterialien, welche aufgrund von Restaurierungsarbeiten am Marmorpalais in Potsdam zur Verfügung standen. Bei den verwitterten Proben des Lasa und Sterzinger Marmors handelt es sich um abgebaute Grabsteine. Die vormalige Exposition und damit auch die jeweilige Verwitterungsgeschichte der Proben ist weitgehend unklar. Das frische Referenzmaterial der polnischen Marmore wurde aus den original Steinbrüchen entnommen, wohingegen die italienischen Marmorproben von Steinmetzen zur Verfügung gestellt wurden.

\subsection{Provenienz, Makrogefüge und Schadensphänomene}

\section{- Carrara Marmor}

Der Carrara Marmor kommt in den Apuanischen Alpen (Alpi Apuane) Italiens (Toskana) vor, nur wenige Kilometer im Hinterland der italienischen Riviera zwischen Genua und Pisa. Hinter dem Handelsnamen Carrara verbergen sich dabei zahlreiche Varietäten dieses Gesteins, die in verschiedenen Steinbrüchen gewonnen werden und sich anhand ihrer Prägung sowie ihrer Gefügemerkmale unterscheiden. Geologisch betrachtet befinden sich die Marmore im unteren Teil der Apenninen Decken, eines metamorphen Komplexes, welcher in Form eines tektonischen Fensters aufgeschlossen ist (CARMINGNANI et al., 1978). Die Überprägung der Marmore ist auf zwei syn-metamorphe Deformationsphasen während der alpinen Gebirgsbildung zurückzuführen (CARMINGNANI, 1990).

In die Untersuchungen wurden drei Proben des Carrara Marmors in unterschiedlichen Erhaltungszuständen, (i) frisch, (ii) mäßig und (iii) stark verwittert, aufgenommen. Beide verwitterten Proben sind durch ein hellweißes Erscheinungsbild mit vereinzelt unregelmäßig schlierenartigem Auftreten von dunkelgrauen Bändern (Marmorierung) gekennzeichnet (Abb. 4.1). Aufgrund ihrer äußeren Merkmale sind sie der Handelsbezeichnung Statuario Carrara zuzuordnen. Die unverwitterte Probe ist durch eine deutlicher hervortretende Marmorierung gekennzeichnet und ist vielmehr mit dem Bianco Carrara CD vergleichbar (vgl. ERTAG, 1980).

Beide verwitterten Proben des Carrara Marmors sind durch eine deutliche Rückwitterung der Oberflächen gekennzeichnet, wobei die Reliefbildung nur schwach ausfällt. Die stark verwitterte Probe weist zusätzlich ein lokal sehr stark ausgeprägtes sandiges Abzuckern der Oberfläche auf, was eine weit fortgeschrittene Gefügeentfestigung anzeigt. 


\section{Carrara (Calcit-Marmor)}

\section{Herkunft}

- Apuanische Alpen Toskana / Italien

\section{Makrogefüge}

- hellweiß mit unregelmäßig schlierenartiger Bänderung, feinkömig

Hauptverwitterungsphänomene

- durchdringende Gefügeentfestigung

Abb. 4.1: Merkmale und makroskopisches Erscheinungsbild der untersuchten verwitterten Probe aus Carrara Marmor (Anschliff).

\section{- Prieborner Marmor}

Das Vorkommen des Prieborner Marmors befindet sich südöstlich von Strzelin (Strelzin) und ca. $1 \mathrm{~km}$ östlich der namensgebenden Ortschaft Przeworno (Prieborn) in Polen. Geologisch befindet sich der Marmor in den Westsudeten. Er ist Teil eines metamorphen Komplexes, der von Granitgneisen, Kalksilikatgesteinen und Glimmer-Sillimanit Schiefern proterozoischen Alters aufgebaut wird. Die Naturwerksteingewinnung des Prieborner Marmors erfolgte nur historisch in einem begrenzten Rahmen. Heute werden nur noch geringe Mengen gefördert und als Marmormehl weiterverarbeitet.

Das äußere Erscheinungsbild des Prieborner Marmors ist durch eine strenge Bänderung gekennzeichnet, die von relativ gleichmäßig schmalen, im Wechsel stehenden hell- und dunkelgrauen Lagen aufgebaut wird (s. Abb. 4.2). Die Mächtigkeit der einzelnen Lagen liegt im mm-Bereich wobei eine Dominanz um $5 \mathrm{~mm}$ festzustellen ist. Auffällig sind vereinzelt vorkommende Risssysteme. Hierbei handelt es sich sowohl um mineralisierte als auch um versiegelte Risse.

\section{Prieborn (Calcit-Marmor)}

\section{Herkunft}

- Przeworno in SW Polen (Schlesien)

Makrogefüge

- hellgrau / grau, engständig streng gebändert, vereinzelt mineralisierte Risse, feinkörnig

\section{Hauptverwitterungsphänomene}

- durchdringende Gefügeentfestigung, lokal starke Rückwitterung präexistierender Risse

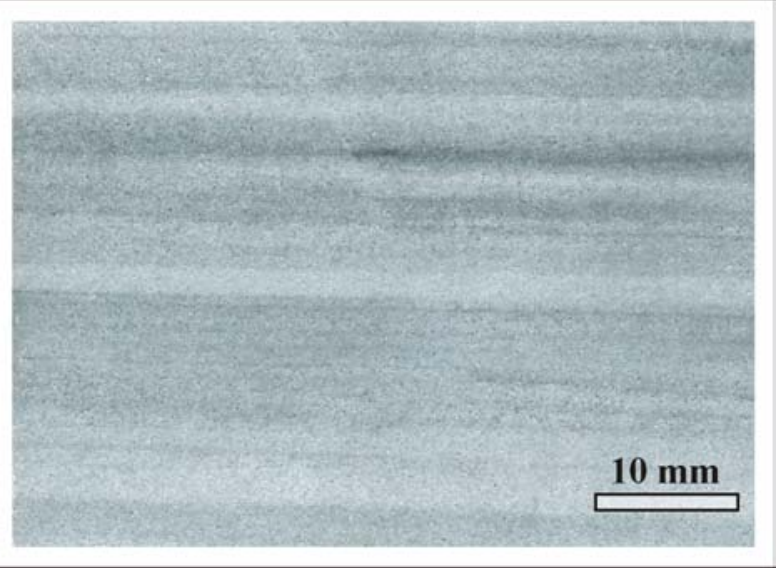

Abb. 4.2: Merkmale und makroskopisches Erscheinungsbild der untersuchten verwitterten Probe aus Prieborner Marmor (Anschliff). 
Der Prieborner Marmor ist durch eine mäßige Verwitterung der Gesteinsoberflächen gekennzeichnet. Dabei ist eine verstärkte Verwitterung an Korngrenzen zu beobachten, welche ein raues Relief erzeugt. In vielen Fällen kann auch ein sandiges Abzuckern der Oberflächen festgestellt werden, was eine Entfestigung des Kornverbandes anzeigt. Weiteres charakteristisches Schadensmerkmal sind verwitterungsbedingte Makrorisse. Hierbei können sowohl herausgewittert (positives Relief) als auch tiefgründig verwitterte offene Risse (negatives Relief) beobachtet werden.

\section{- Lasa Marmor}

Die Vorkommen des Lasa Marmors befinden sich im Vinschgau-Ultental südlich von Lasa in Südtirol/Italien. Geologisch befinden sie sich im tektonischen Verband des komplexen ostalpinen Deckenbaus und sind Teil des Schneeberger Zuges. Die Marmorlagen sind eingeschaltet in eine schmale ost-west-streichende Bunte-Serie des Campo-Kristallins und liegen in Wechsellagerung mit Glimmerschiefern und Amphiboliten. Von wirtschaftlichem Interesse sind lediglich Bereiche von bis $\mathrm{zu} 100 \mathrm{~m}$ mächtigen Marmorlagern, wobei sich gegenwärtig der Abbau auf zwei Brüche, den sog. Göflander- und den Weisswasserbruch, konzentriert.

Die in die Untersuchungen aufgenommene frische wie auch die verwitterte Probe des Lasa Marmors sind der Handelssorte Bianco statuario di Lasa (Lasa Marmor reinweiß gefleckt) zuzuordnen. Der makroskopische Charakter des Gesteins ist durch die leuchtend weiße Erscheinung gekennzeichnet, wobei eine weitere Strukturierung vollständig fehlt (Abb. 4.3).

\section{Lasa (Calcit-Marmor)}

Herkunft

- Lasa im Vinschgautal Südtirol / Italien

Makrogefüge

- hellweiß, mittelkörnig

Hauptverwitterungsphänomene

- durchdringende Gefügeentfestigung

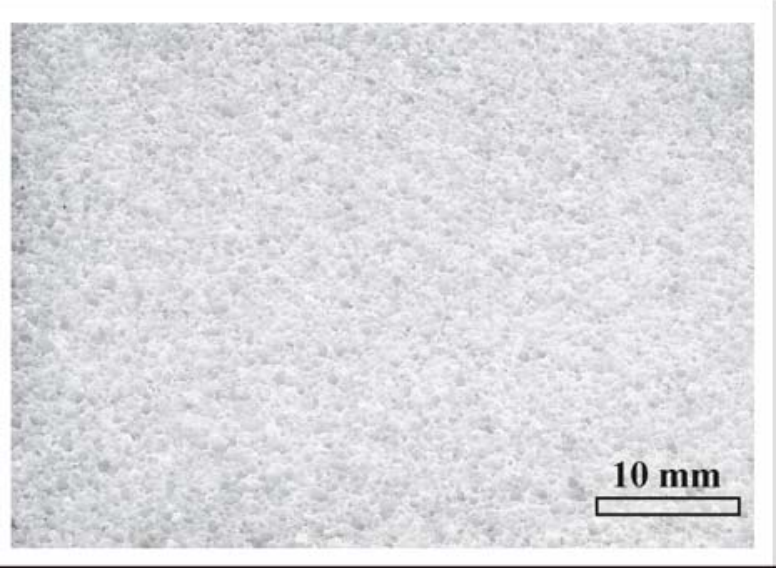

Abb. 4.3: Merkmale und makroskopisches Erscheinungsbild der untersuchten verwitterten Probe aus Lasa Marmor (Anschliff).

Auffällig ist die leicht zu erkennende gröbere Körnung im Vergleich zum Carrara Marmor. Das Verwitterungsbild der exponierten Probe, eines abgebauten Grabsteins, ist durch eine deutliche Rückwitterung der Oberfläche gekennzeichnet. Makroskopische Rissbildungen fehlen ebenso wie eine durchdringende Gefügeauflockerung, womit die Probe als relativ gering verwittert angesehen werden kann. 


\section{- Grosskunzendorfer Marmor}

Das Vorkommen dieses Marmors befindet sich nahe der Ortschaft Slawniowice (Grosskunzendorf) im südwestlichen Teil Polens wenige Kilometer südlich der Stadt Nysa (Neisse). Geologisch befindet sich die Lagerstätte in den Ostsudeten. Der Grosskunzendorfer Marmor wird zur Desna-Formation gezählt, welche von Glimmerschiefern, Paragneisen, Migmatiten, Amphiboliten und Marmoren aufgebaut wird. Die Deformationsgeschichte umfasst eine prävariszische sowie variszische Überprägung.

Der Marmor ist weitgehend homogen hellgrau (Abb. 4.4), nur vereinzelt ist eine dunkelgraue Foliation anhand von Glimmerlagen nachgezeichnet. Die verwitterte Probe ist neben einer deutlichen Rückwitterung der Oberfläche vor allem durch starke Rissbildung gekennzeichnet. Hierbei handelt es sich um parallel verlaufende weit geöffnete Makrorisse. Daneben ist lokal auch ein Herausbrechen einzelner Körner festzustellen.

\section{Grosskunzendorf (Calcit-Marmor)}

\section{Herkunft}

- Slawniowice SW Polen / Schlesien

Makrogefüge

- hellgrau, grobkörnig erscheinend

Hauptverwitterungsphänomene

- durchdringende Gefügeentfestigung an

Spaltflächen und Korngrenzen, lokale

Öffnung von transgranularen Rissen

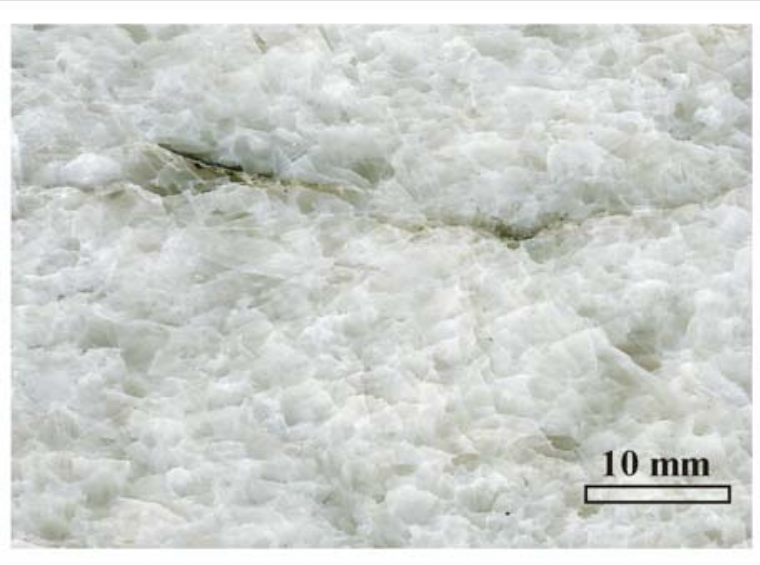

Abb. 4.4: Merkmale und makroskopisches Erscheinungsbild der untersuchten verwitterten Probe aus Grosskunzendorfer Marmor (Anschliff).

\section{- Sterzinger Marmor}

Das Vorkommen des Sterzinger Marmors befindet sich auf der Töll am Beginn des Vinschgau im Ratschingstal westlich von Vipiteno (Sterzing) in Südtirol/Italien. Geologisch liegt das Marmorvorkommen ebenfalls wie der Lasa Marmor im Schneeberger Zug und ist somit dem Ostalpinen Deckenbau zuzuordnen. Der Marmor ist eingeschaltet in Granatglimmer- und Hornblendegarbenschiefer.

Makroskopisch ist der Sterzinger Marmor weitgehend mit dem Grosskunzendorfer Marmor vergleichbar. Sowohl das äußere Erscheinungsbild wie auch die Verwitterungsphänomene weisen Parallelen auf. So zeigt der Sterzinger Marmor ein graues Erscheinungsbild, wobei die Grauintensität lokal nuanciert (Abb. 4.5). Auffallend ist die typische grobe Körnung. 


\section{Sterzing (Calcit Marmor)}

\section{Herkunft}

- Vipiteno am Brenner Südtirol / Italien Makrogefüge

- hellgrau, grobkörnig erscheinend

Hauptverwitterungsphänomene

- durchdringende Gefügeentfestigung an Spaltflächen und Korngrenzen, lokale Öffnung von transgranularen Rissen

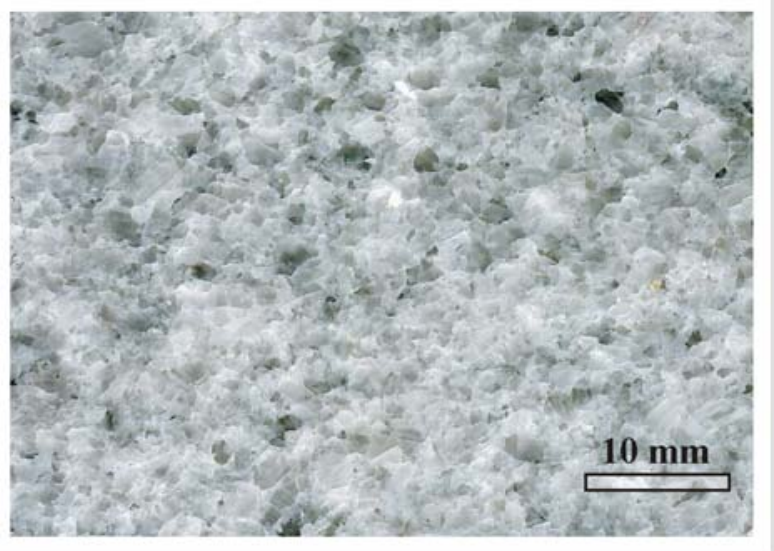

Abb. 4.5: Merkmale und makroskopisches Erscheinungsbild der untersuchten verwitterten Probe aus Sterzinger Marmor (Anschliff).

Als Verwitterungsphänomene können eine penetrative Rissentfestigung an Spaltflächen und Korngrenzen ermittelt werden. Nur sehr vereinzelt treten das Gestein durchziehende isolierte offene Risse auf, an denen das Gestein zerbrechen kann.

\section{- Kauffunger Marmor}

Der Kauffunger Marmor tritt in verschiedenen Aufbrüchen in der Nähe der namensgebenden Ortschaft Wojcieszów (Kauffungen) in Polen zu Tage. Er bildet zusammen mit Metavulkaniten und feinklastischen Metasedimenten einen Teil des Bober-Katzbach-Gebirges in den Westsudeten. Die Gesteine des paläozoischen Grundgebirges weisen grün- bis blauschieferfazielle Überprägungen auf. Der derzeitige Abbau des Kauffunger Marmors findet nur noch zur Gewinnung von Marmormehl statt (DAHLGRÜN, 1934).

Der Marmor hebt sich deutlich durch sein rote Färbung von den weiteren Marmortypen ab (Abb. 4.6). Das sehr heterogene Gestein besteht aus einer grauen, leicht rötlichen, feinkörnigen Calcit-Matrix welche von gelbrötlichen feinkörnigen und sehr unregelmäßigen Dolomitadern durchzogen wird. Aufgrund der höheren Verwitterungsresistenz, formt der Dolomit ein positives Relief auf der Oberfläche.

\section{Kauffung (Calcit-Marmor)}

Herkunft

- Wojcieszów in SW Polen (Schlesien)

Makrogefüge

- rötlich bis grau, engständige Durchäderung verschiedener mineralisierter Risssysteme, feinstkörnig

\section{Hauptverwitterungsphänomene}

- lokale Entfestigung der präexistierenden Risse

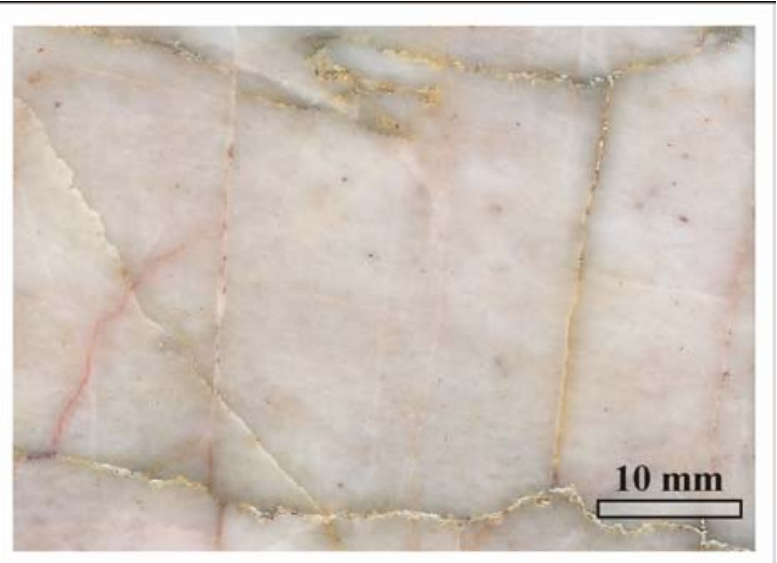

Abb. 4.6: Merkmale und makroskopisches Erscheinungsbild der untersuchten verwitterten Probe aus Kauffunger Marmor (Anschliff). 
Die wesentlichen makroskopischen Gefügeelemente sind verschiedene Systeme parallel angelegter Risse. Hierbei können sowohl offene als auch mit verschiedenen Mineralphasen versiegelte Risse beobachtet werden. Für letztere ist eine Variation der Mächtigkeiten von einigen $\mathrm{mm}$ bis $\mathrm{zu} \mathrm{cm}$ Größe festzustellen. Sie sind vorwiegend mit unterschiedlich gekörntem Calcit, Dolomit zum Teil auch Quarz und untergeordnet Hämatit gefüllt.

Die Matrix des Gesteins zeigt makroskopisch keine erkennbare Gefügeauflockerung. Die Entfestigung erfolgt über die verschiedenen Risssysteme in Form des Ausbrechens von Teilstücken.

\subsection{Gefügeinventar}

Das Gefügeinventar der untersuchten Marmore beinhaltet sowohl ursprüngliche als auch verwitterungsbedingte Gefügemerkmale. Bei den ursprünglichen Gefügen handelt es sich um Merkmale aus der geologischen Vergangenheit, also um das eigentliche Gesteinsgefüge. Hierzu gehören alle relevanten Elemente, die durch die Genese des Gesteins erzeugt wurden, wie Korngefüge, Kornformregelung, Textur etc. Unter die verwitterungsbedingten Merkmale fallen offene Mikrorisse und ihre Modifikation durch chemische Lösungsprozesse. Letztere werden aufgrund ihrer Aussagefähigkeit bezüglich der Verwitterungsprozesse im Folgenden gesondert betrachtet.

\subsubsection{Mikrogefüge}

Die untersuchten Marmortypen weisen eine große Vielfalt und Variabilität von Gefügeelementen auf. Es kann jedoch festgestellt werden, dass sich einige Marmore gleichen, also verwandte bzw. ähnliche Gefügecharakteristika aufweisen. Dabei ermöglichen bestimmte Hauptgefügemerkmale eine Untergliederung in verschiedene Gefügetypen (Korngefüge- und Texturtypen). Die Hauptgefüge können direkt und indirekt einen signifikanten Einfluss auf die physikalischen und mechanischen Eigenschaften der Gesteine ausüben. Dementsprechend ist für die Gefügetypen ein differentes petrophysikalisches Verhalten festzustellen.

Eine wesentliche Gliederung kann über die Einteilung in Korngefügetypen erfolgen. Hierbei ermöglichen die Kornform bzw. das Korngrenzengefüge und die Korngrößenverteilung die Unterteilung der untersuchten Marmore in drei unabhängige Typen (s. Abb.4.7). Typ I repräsentiert equigranulare Marmore mit polygonalen Kornformen. Vertreter dieses Korngefügetyps sind der Carrara, der Prieborner und der Lasa Marmor. Der Grosskunzendorfer und der Sterzinger Marmor sind zu Typ II zu zählen, welcher durch eine ungleichmäßige (seriate) Korngrößenverteilung und durch interlobate Kornformmerkmale gekennzeichnet ist. Typ III stellt einen Sonderfall dar, der durch den Kauffunger Marmor vertreten wird. Dieser Typ zeichnet sich durch ein heterogenes Korngefüge mit großen reliktischen Altkörnern aus, die von einer Matrix aus vorwiegend polygonalen Subkörnern und feinen rekristallisierten Körnern umgeben sind. Dabei ist die Korngrößenverteilung ungleichmäßig (vorwiegend seriate, selten unequigranular). 


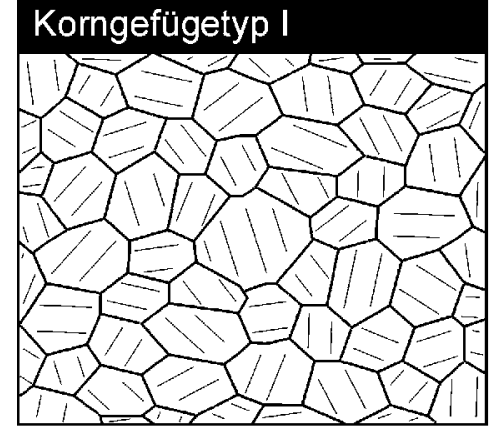

equigranular polygonal

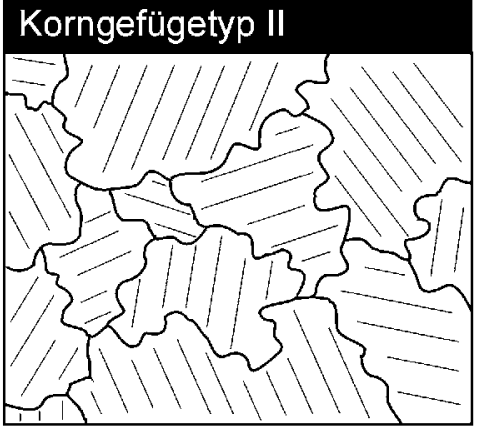

seriate interlobat

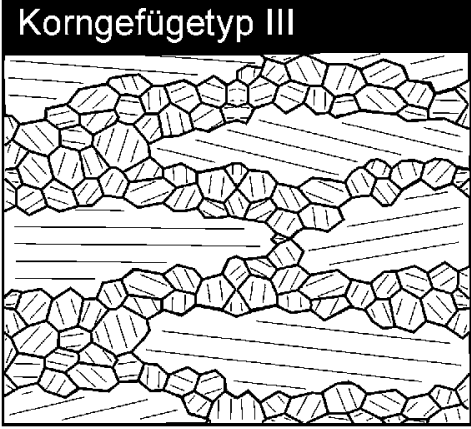

seriate polygonal/interlobat

Abb. 4.7: Schematische Darstellung idealisierter Korngefügetypen der untersuchten Marmore.

Bezüglich einer Gliederung in Texturtypen können die Endglieder der in Marmoren vorkommenden kristallographischen Vorzugsregelung herangezogen werden. Dies sind der cAchsen- und der a-Achsenfasertyp (vgl. Kap. 2.2). Zwar repräsentieren die Texturen der untersuchten Marmore modifizierte Übergangsmuster jedoch sind generell deutliche Tendenzen zum jeweiligen Endglied festzustellen, die sich auch in den jeweiligen petrophysikalischen Eigenschaften äußern. Eine große Bedeutung für das Materialverhalten kommt der Intensität der Textur zu. Für die untersuchten Marmore kann dabei eine relative Einteilung in schwache, mittlere und starke Textur erfolgen.

\section{- Carrara Marmor (Korngefügetyp I)}

Im mikroskopischen Skalenbereich weist der Carrara Marmor ein charakteristisch granoblastisches Korngefüge auf (Tafel 1, Abb. 1), welches eine statische Rekristallisation als wesentlichen gefügebildenden Prozess anzeigt. Dabei können zwei verschiedenartige Gefügebereiche voneinander abgegrenzt werden. Dies ist eine relativ homogene Matrix, welche die makroskopisch hellweißen Bereiche darstellt und lagenartig bis fleckenhaft lokal auftretende sehr feinkörnige Domänen, welche die dunkleren Partien des Gesteins repräsentieren. Letztere zeigen Verwachsungen von Dolomit/Calcit und unterscheiden sich damit auch mineralogisch von der Matrix die fast ausschließlich von Calcit gebildet wird.

Das Korngefüge der homogenen Matrix repräsentiert ein equigranulares Kornaggregat mit vorwiegend polygonalen Kornformen (Abb.4.8a,b). Die mittlere Korngröße liegt für die untersuchten Proben zwischen $140 \mu \mathrm{m}$ und $170 \mu \mathrm{m}$. Die Ausbildung der Korngrenzen ist gerade bis leicht geschwungen (Tafel 2, Abb. 1). In Teilbereichen ist das Korngefüge durch vollständig equilibrierte Korngrenzen (,foam structure“) gekennzeichnet, welches einen Erholungsprozess durch Korngrenzen/flächen Reduktionsprozesse anzeigt (vgl. PASSCHIER \& TrOUW, 1996). Nur lokal können auch buchtige Korngrenzengeometrien beobachtet werden. Auffällig ist eine relativ seltene Ausbildung von Zwillingen.

Für die Proben aus Carrara Marmor ist generell eine deutliche Kornformregelung festzustellen. Sie wird in der YZ- und in der XY-Ebene nachgezeichnet anhand einer bevorzugten Orientierung der Korngrenzen parallel zur Y-Richtung (Abb. 4.8c). Damit ergibt sich ein dreidimensionales Ellipsoid als bevorzugte Kornform. Die Textur der untersuchten Carrara Marmore ist generell schwach (unter 2,0 VGV) und weist das Muster eines cAchsenfasertyps auf. Die in Abb. 4.8d dargestellte Textur der stark verwitterten Probe weist 


\section{Carrara}

\section{Korngefüge}

- equigranular polygonal

Mittlere Korngröße

- ca. $170 \mu \mathrm{m}$

Korngrenzengeometrie

gerade bis geschwungen

\section{Korngrenzenregelung}

- parallel der Y-Richtung in der XY- und YZ-Ebene

\section{Textur}

- c-Achsenfasertyp schwacher Intensität

\section{Korngefüge}

a)
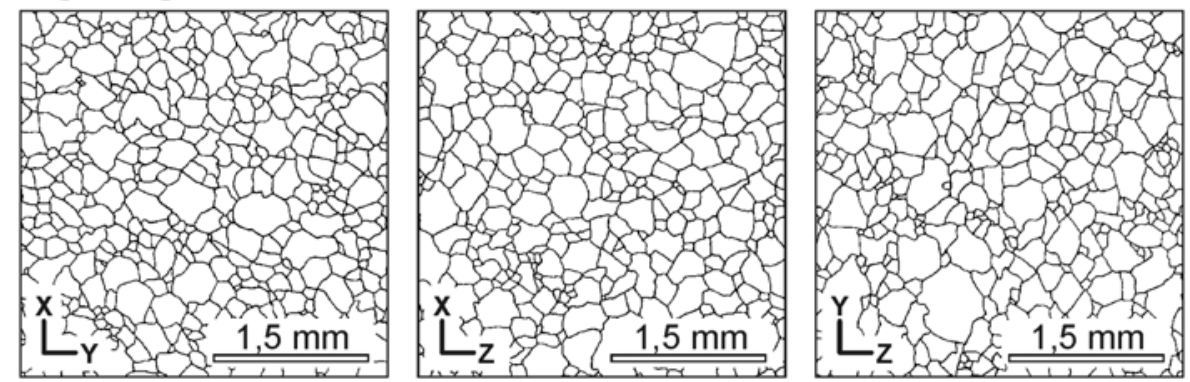

Kornflächenverteilung

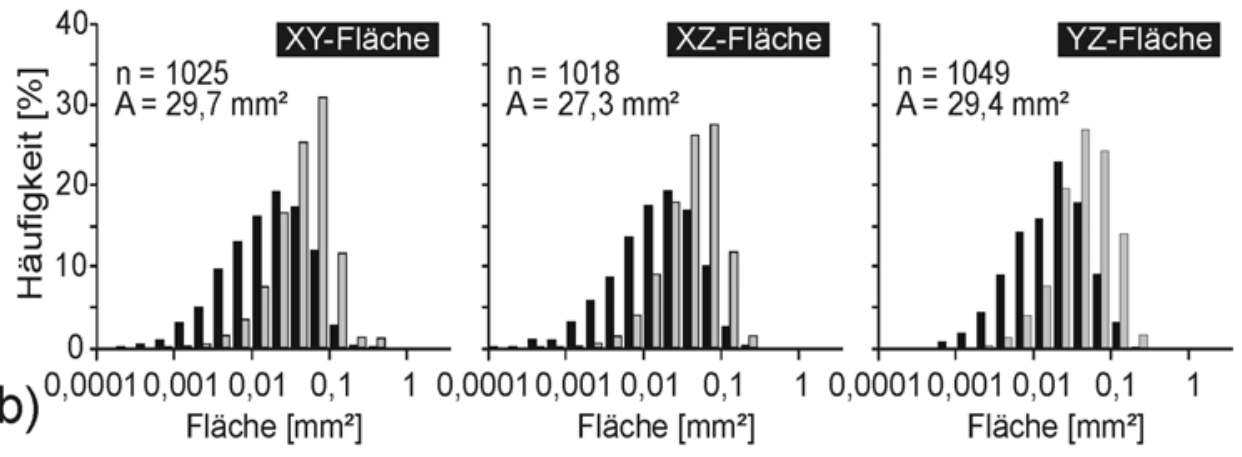

Korngrenzenregelung

c)

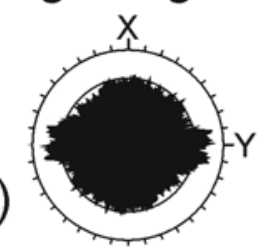

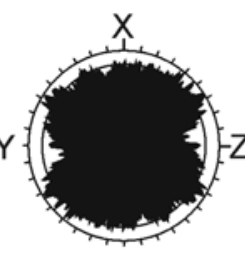

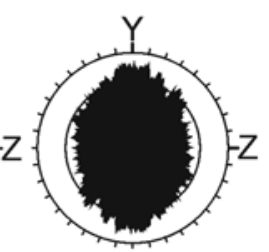

\section{Textur}

c (001)

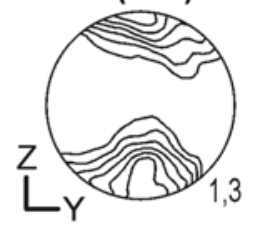

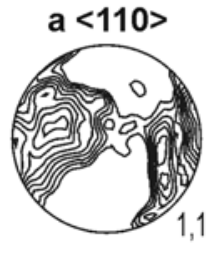
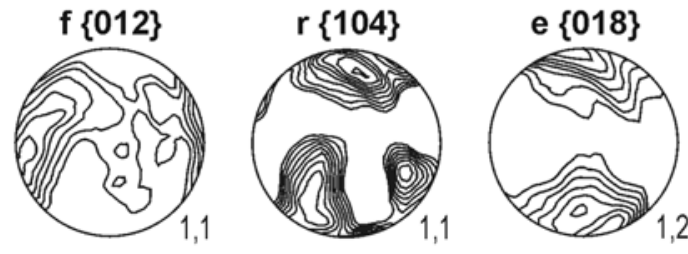

Abb. 4.8: Wesentliche Gefügemerkmale der drei orthogonalen Schnittlagen der verwitterten Probe aus Carrara Marmor: a) Korngefüge, b) Korngrößenverteilung, dargestellt als Flächenparameter ( $\mathrm{n}=$ Anzahl und A = Gesamtfläche der analysierten Körner), c) bevorzugte Orientierung der Korngrenzen und d) Textur (Intensität in VGV = Vielfaches der Gleichverteilung). 
abweichend vom reinen Fasertyp ein mehr oder weniger deutlich ausgeprägtes a-Achsen Punktmaximum auf.

Nach KL-Untersuchungen ist für die Calcitkristalle der Matrix eine weitgehend homogen dunkelbraune Lumineszenzfarbe festzustellen. Nur lokal können hellbraun bis dunkelbraun lumineszierende Domänen beobachtet werden, welche zumeist einen deutlich geometrischen Charakter in Form von Streifungen aufweisen (Tafel 3, Abb. 1). Vermutlich handelt es sich hierbei um Reliktgefüge schalenbildender Organismen. Diese nur unvollständige Homogenisierung der KL-Eigenschaften des Marmors weist für die untersuchten Proben auf eine relativ schwache metamorphe Überprägung des Gesteins hin.

Die lokal auftretenden sehr feinkörnigen Domänen zeichnen sich mikroskopisch durch eine schwer aufzulösende, komplexe Verwachsung von verschiedenen Kristallindividuen aus. Dolomit und Calcit weisen zumeist polygonale Kornformen auf, wobei sich die Korngröße im Bereich unterhalb $30 \mu \mathrm{m}$ befindet. Die Korngrenzen sind hier mit Flüssigkeits- und feinsten Mineraleinschlüssen dekoriert. Die Dolomit-Kristalle sind durch eine pinkfarbene Lumineszenz charakterisiert und lassen sich somit von den Calcit-Kristallen mit bräunlicher Lumineszenz im KLM leicht voneinander abgrenzen (Tafel 3, Abb. 1). In diesen Gefügebereichen dominiert Dolomit wobei lokal Partien vorkommen, in denen Calcit vollständig zurücktritt.

\section{- Prieborner Marmor (Korngefügetyp I)}

Das Mikrogefüge des Prieborner Marmors weist die typische granoblastische Ausbildung des Korngefügetyps I auf (Tafel 1, Abb. 2) und zeigt, vergleichbar wie dem Carrara Marmor, statische Rekristallisation als wesentlichen gefügeprägenden Prozess an. Im Mikrobereich ist der Marmor durch ein relativ homogenes Gefüge mit vereinzelt auftretenden sehr feinkörnigen Bändern gekennzeichnet. Es handelt sich um einen reinen Calcit Marmor, der akzessorisch Pyrit und Quarz beinhaltet.

Charakteristisch ist das equigranulare Korngefüge des Marmors mit polygonalen Kornformen (Abb. 4.9a,b). Hierbei sind die Korngrenzen in der Mehrzahl der Fälle etwas stärker geschwungen in Form eines engständigem Wellenmusters (Tafel 2, Abb. 2). Ebenfalls kann dieser Marmor aber auch lokal ein equilibriertes Korngrenzengefüge mit geradlinig verlaufenden Kornrändern aufweisen. Die mittlere Korngröße liegt um $160 \mu \mathrm{m}$ für die verwitterte Probe, wohingegen die frische Probe um ca. $300 \mu \mathrm{m}$ liegt. Zwillingsbildung ist häufiger als beim Carrara Marmor zu beobachten jedoch immer noch recht selten. Die Zwillingslamellen haben einen geraden und parallelen Verlauf und durchziehen die Calcit Körner vollständig. Die Korngrenzen erscheinen zumeist geöffnet und sind häufig mit feinstkörnigen Partikeln, vermutlich Graphit, dekoriert. Die sehr vereinzelt auftretenden Bänder feinerer Körnung heben sich mit Korngrößen um $50 \mu \mathrm{m}$ deutlich von der Matrix ab. Innerhalb dieser Lagen ist Pyrit als Nebengemengteil, häufig in Form von bis zu $20 \mu \mathrm{m}$ großen, würfelförmigen Kristallen, enthalten. Die Lagen verlaufen diagonal zur Foliation. 
Prieborn

\section{Korngefüge}

- equigranular polygonal

Mittlere Korngröße

- ca. $160 \mu \mathrm{m}$

Korngrenzengeometrie

gerade bis geschwungen

\section{Korngrenzenregelung}

- in der XY- und der YZ-Ebene senkrecht zur Foliation drehend

\section{Textur}

- c-Achsenfasertyp starker Intensität

Korngefüge

a)
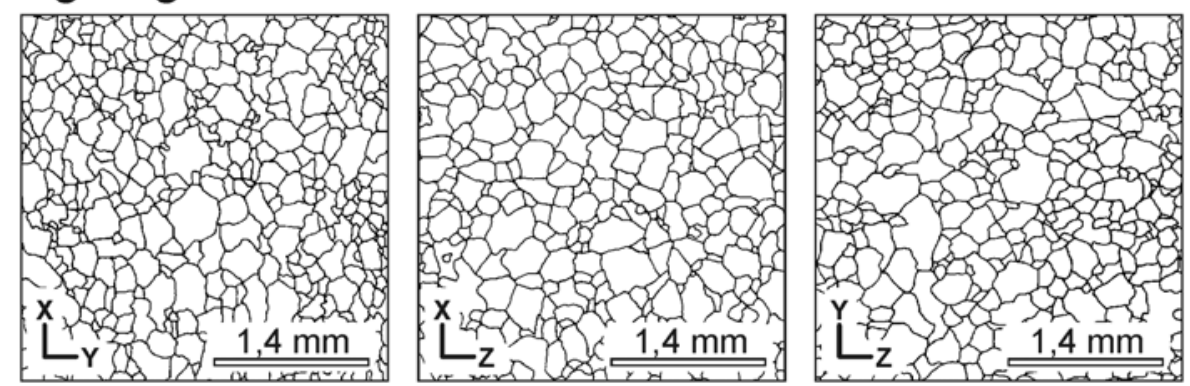

Kornflächenverteilung

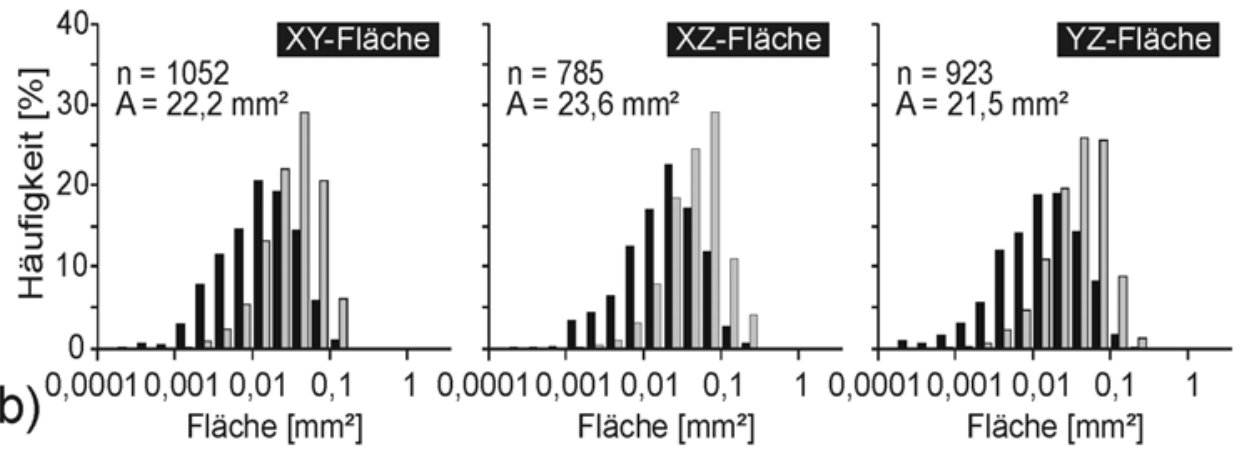

Korngrenzenregelung

C)

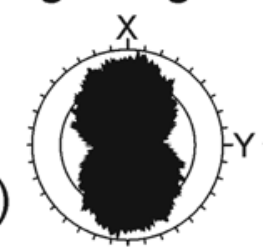

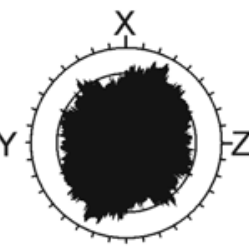

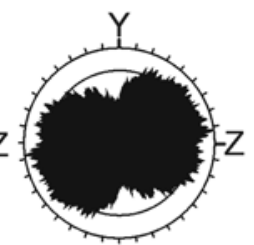

Textur

d)
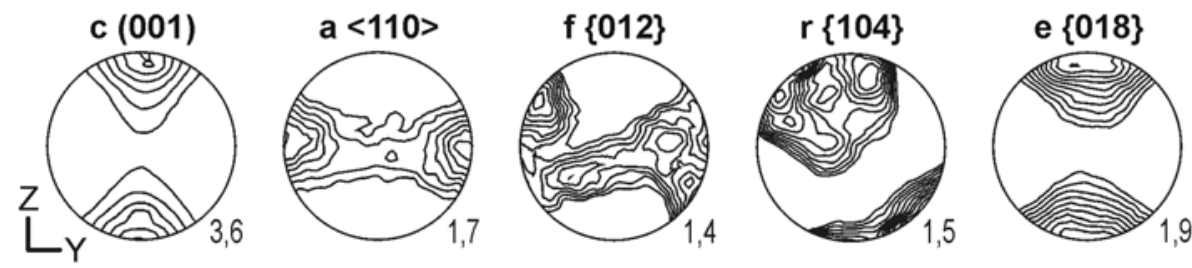

Abb. 4.9: Wesentliche Gefügemerkmale der drei orthogonalen Schnittlagen der verwitterten Probe aus Prieborner Marmor: a) Korngefüge, b) Korngrößenverteilung, dargestellt als Flächenparameter ( $\mathrm{n}=$ Anzahl und $\mathrm{A}=$ Gesamtfläche der analysierten Körner), c) bevorzugte Orientierung der Korngrenzen und d) Textur (Intensität in VGV = Vielfaches der Gleichverteilung). 
Anhand einer Korngrenzenregelung wird für die in Abb. 4.9c dargestellte verwitterte Probe eine bevorzugte diskusförmige Kornform nachgezeichnet. Auffallend ist dabei, dass die Kornformregelung senkrecht in der Foliation steht. Die bevorzugte Orientierung von Korngrenzen verläuft dabei parallel zur Z-Richtung in der YZ-Ebene und parallel der XRichtung in der XY-Ebene. Die Textur für beide untersuchten Proben ist durch einen stark ausgeprägten c-Achsenfasertyp gekennzeichnet. Für die in Abb. 4.9d dargestellte verwitterte Probe liegt die Intensität der c-Achsen Regelung relativ hoch bei 3,5 VGV, wobei eine deutliche Auslängung des Punktmaximums zu beobachten ist. Verbunden hiermit ist ein ausgeprägtes Submaximum auf dem a-Achsengürtel.

Ein weiteres Gefügemerkmal des Prieborner Marmors ist eine starke Alteration der Kornränder. Die äußeren Bereiche der Körner sind durch eine intensiv gelbe Lumineszenz gekennzeichnet, die sich deutlich von der schwach rötlichen KL-Farbe der Kornzentren abhebt (Tafel 3, Abb. 2). Vom Kornrand zum Zentrum wird die Alteration schwächer, wodurch ein Zonarbau mit Kernstruktur erkennbar wird. Diese Gefügemerkmale können sowohl für die exponierte als auch für die frische Marmorprobe ermittelt werden, womit die Alteration der Kristalle auf einen Fluiddurchstrom schon in der Lagerstätte zurückzuführen ist.

\section{- Lasa Marmor (Korngefügetyp I)}

Der Lasa Marmor lässt sich zu den granoblastischen Marmorvarietäten zählen (s. Tafel 1, Abb. 3). Es handelt sich um einen reinen Calcit Marmor, welcher akzessorisch Quarz beinhaltet.

Das Korngefüge des Marmors ist vorwiegend equigranular (Abb. 4.10a,b). Nur lokal treten Bereiche auf bei denen deutliche Korngrößenunterschiede hervortreten. Die Korngrenzen sind zumeist geschwungen bis schwach buchtig (Tafel 2, Abb. 3). Lokal sind jedoch auch deutlicher verzahnte Korngrenzen zu beobachten. Im Gegensatz zum Prieborner und Carrara Marmor weist der Lasa Marmor eine größere mittlere Korngröße auf, welche bei den untersuchten Proben um ca. $400 \mu \mathrm{m}$ liegt. Weiterhin ist auch ein relativ häufiges Auftreten von Zwillingslamellen festzustellen.

Eine bevorzugte Korngrenzenorientierung verläuft in der YZ-Ebene parallel zur Z-Richtung und in der XY-Ebene parallel zur X-Richtung (Abb. 4.10c). Die bevorzugte Kornform ist somit diskusförmig und damit vergleichbar zum Prieborner Marmor ausgebildet. Bezüglich der Textur stellt der Lasa Marmor mit Ausbildung eines a-Achsenfasertyps eine Ausnahme für die untersuchten Marmore dar (Abb. 4.10d). Hierbei sind beide untersuchten Proben auch bezüglich der Intensität um 2,0 VGV für das c-Achsenmaximum vergleichbar.

Das KL-Inventar wird für die meisten untersuchten Proben durch eine dunkelbraune bis schwach rotbraune Lumineszenz dominiert. Lagenartig kann jedoch lokal eine starke Alteration der Calcit-Kristalle beobachtet werden, welche anhand hellbrauner bis gelblicher Lumineszenz erkennbar ist (s. Tafel 3, Abb. 3). Dabei ist häufig ein Zonarbau festzustellen, wobei ein gradueller Übergang von hellen zu dunklen KL-Farben von den Korngrenzen zum Zentrum der Körner ausgebildet ist. Nicht lumineszierende Quarzkörner sind mit dem Auftreten der Lagen hellerer Lumineszenz assoziiert. 


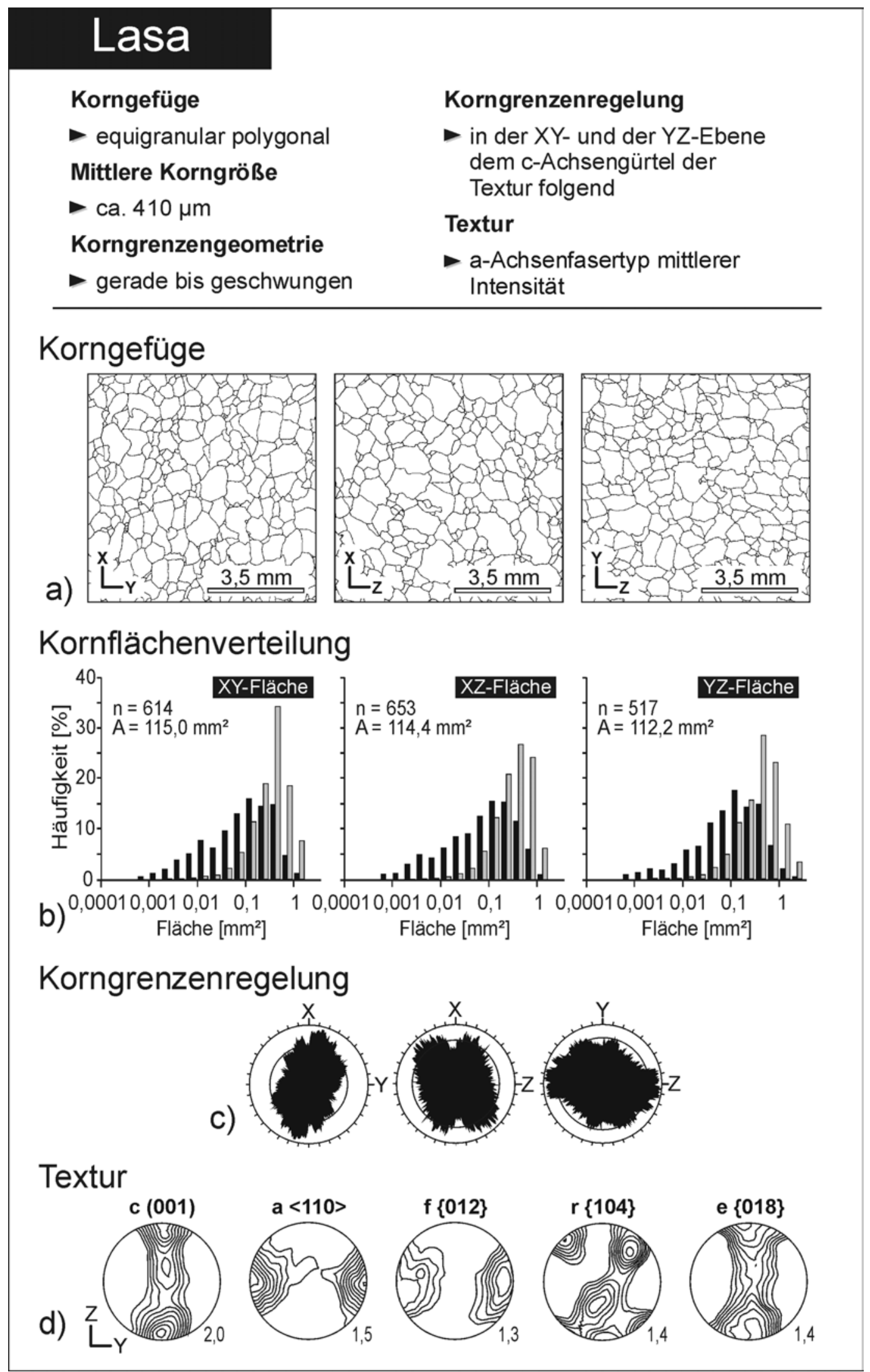

Abb. 4.10: Wesentliche Gefügemerkmale der drei orthogonalen Schnittlagen der verwitterten Probe aus Lasa Marmor: a) Korngefüge, b) Korngrößenverteilung, dargestellt als Flächenparameter ( $\mathrm{n}=$ Anzahl und $\mathrm{A}=$ Gesamtfläche der analysierten Körner), $\mathrm{c}$ ) bevorzugte Orientierung der Korngrenzen und d) Textur (Intensität in VGV = Vielfaches der Gleichverteilung). 


\section{- Grosskunzendorfer Marmor (Korngefügetyp II)}

Der Grosskunzendorfer Marmor ist ein grobkörniger Calcit Marmor dessen Mikrogefüge durch eine postmetamorphe kaltplastische Deformation charakterisiert ist und als schwach kataklastisch beschrieben werden kann (vgl. Tafel 1, Abb. 4). Akzessorisch tritt in den untersuchten Proben Biotit und Quarz auf. Beide können aber auch mit einem deutlich höheren Anteil am Gesteinsaufbau des Grosskunzendorfer Marmors beteiligt sein (vgl. WEISS et al., 1999).

Das Mikrogefüge des Gesteins ist durch eine ungleichmäßige (seriate) Korngrößenverteilung mit einer mittleren Korngröße um $620 \mu \mathrm{m}$ gekennzeichnet (s. Abb. 4.11a,b). Der Marmor erscheint dabei im mikro- und auch makroskopischen Skalenbereich grobkörniger, jedoch wird die mittlere Korngröße durch die hohe Anzahl kleiner Körner stark herabgesetzt. Die Korngrenzengeometrie, insbesondere der bis zu $3 \mathrm{~mm}$ erreichenden Großkörner, ist stark buchtig bis verzahnt (Tafel 2, Abb.4). Daraus resultiert ein interlobates Kornaggregat, welches auf Korngrenzenmigration als bedeutenden gefügeprägenden Deformationsmechanismus hinweist.

Die Calcit-Kristalle weisen sehr häufig Zwillingsbildung auf, zumeist in Form polysynthetischer Zwillinge, die an ihrer engständigen, lamellenartigen Abfolge erkennbar sind (vgl. Tafel 1, Abb. 4). Vielfach treten kaltplastische Verformungsgefüge wie undulöse Auslöschung, Deformationsbänder, Subkörner und gebogene Zwillingslamellen auf, welche als Resultat einer späten Deformationsphase zu interpretieren sind. Es kann davon ausgegangen werden, dass diese Gefügemerkmale einen signifikanten Einfluss auf die physikalischen Eigenschaften des Gesteins ausüben.

Eine bevorzugte Orientierung der Korngrenzen ist nur in der XZ-Ebene parallel zur XRichtung festzustellen (Abb. 4.11c). Die Textur der in Abb. 4.11d dargestellten verwitterten Probe repräsentiert einen c-Achsenfasertyp mit relativ starker Intensität von 3,7 VGV des cAchsenmaximums (Abb. 4.11d). Diese Marmorprobe weist auch die stärkste Regelung der eFlächen $\{018\}$ der untersuchten Marmore auf. Die frische Probe zeigt ein vergleichbares Texturmuster wobei die Intensität etwas schwächer ausfällt.

Das Lumineszenzverhalten der untersuchten Proben zeigt eine vollständige Homogenisierung des KL-Inventars durch die metamorphe Überprägung an. Dabei ist eine nur schwache violette Lumineszenz festzustellen (s. Tafel 3, Abb. 4).

\section{- Sterzinger Marmor (Korngefügetyp II)}

Der Sterzinger Marmor repräsentiert einen grobkörnigen Calcit Marmor mit Gefügemerkmalen einer späten kaltplastischen Deformationsphase (vgl. Tafel 1, Abb. 5). Er weist damit auch im Mikrogefüge Ähnlichkeiten zum Grosskunzendorfer Marmor auf.

Der Sterzinger Marmor ist durch ein seriates Korngefüge gekennzeichnet, wobei die Körner vorwiegend irreguläre Kornformen mit stark buchtigen, verzahnten Korngrenzen aufweisen (Abb. 4.12a,b; Tafel 2, Abb. 5). Die Körner zeigen sehr häufig Zwillingsbildung, mit vielfach stark verbogenen Lamellen. Weiterhin treten verbreitet kaltplastische Verformungsgefüge wie undulöse Auslöschung und Subkornbildung auf. Diese Gefüge weisen auf eine Deformation 


\section{Grosskunzendorf}

\section{Korngefüge}

- seriate interlobat

Mittlere Korngröße

- ca. $620 \mu \mathrm{m}$

Korngrenzengeometrie

buchtig bis verzahnt

\section{Korngrenzenregelung}

- parallel X-Richtung (Foliation) in der XZ-Ebene

Textur

c-Achsenfasertyp starker

Korngefüge

a)
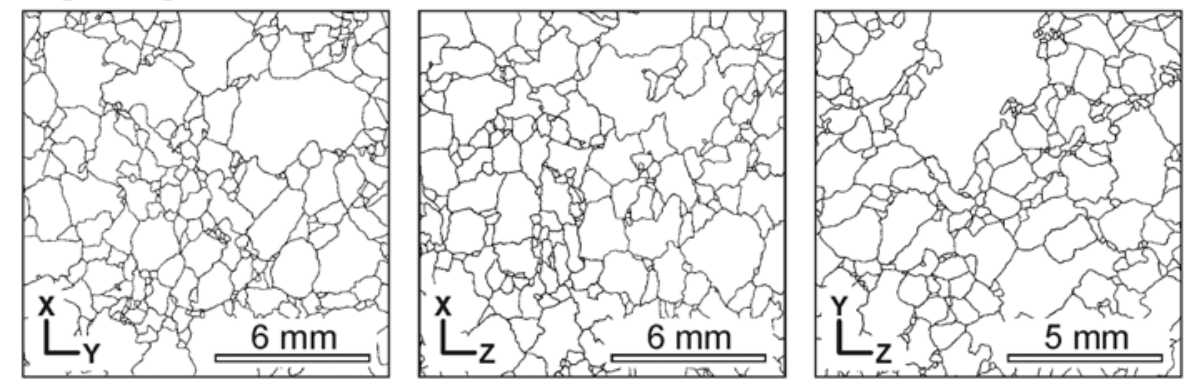

Kornflächenverteilung
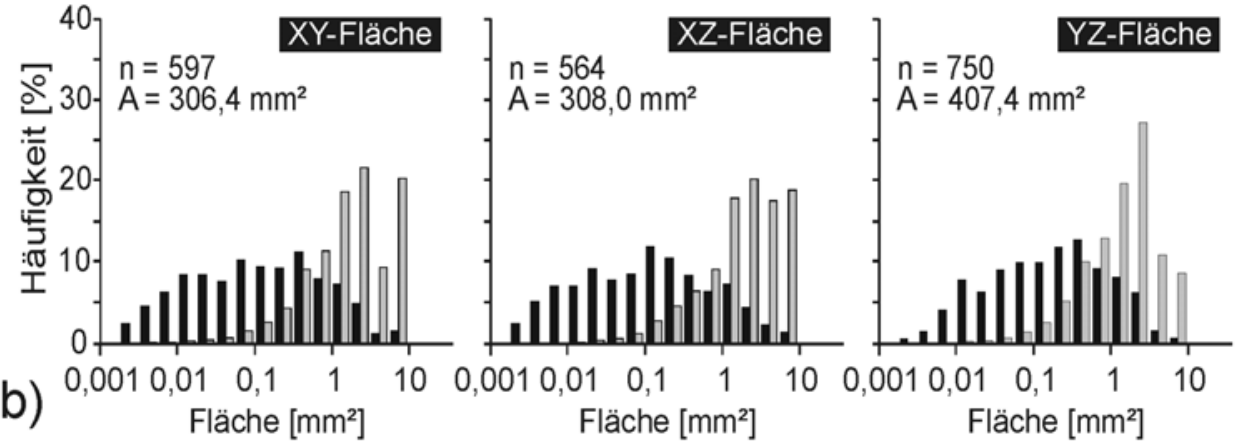

Korngrenzenregelung

c)

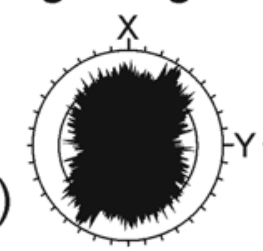

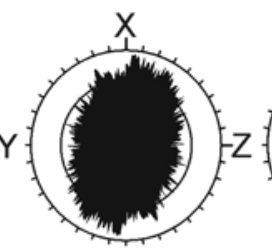

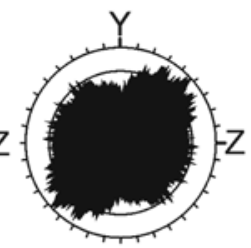

Textur

d)
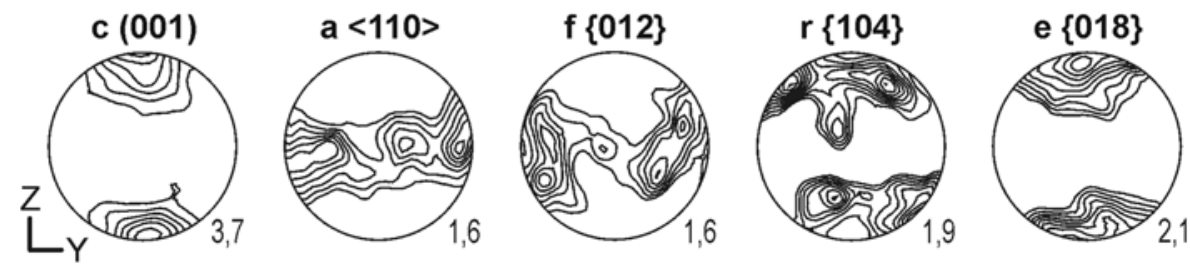

Abb. 4.11: Wesentliche Gefügemerkmale der drei orthogonalen Schnittlagen der verwitterten Probe aus Grosskunzendorfer Marmor: a) Korngefüge, b) Korngrößenverteilung, dargestellt als Flächenparameter ( $\mathrm{n}=$ Anzahl und $\mathrm{A}=$ Gesamtfläche der analysierten Körner), $\mathrm{c}$ ) bevorzugte Orientierung der Korngrenzen und d) Textur (Intensität in VGV = Vielfaches der Gleichverteilung). 
des Gesteins im späten Entwicklungsstadium hin. Daneben sind die verzahnten Korngrenzen ein wesentliches Merkmal einer Korngrenzen-Migrationsrekristallisation (PASSCHIER \& Trouw, 1996). Als weitere Mineralphase tritt vereinzelt Biotit auf, welcher mit typisch tafelförmigem Habitus auf Korngrenzen aber auch innerhalb der Calcit-Kristalle eingeschlossen vorkommt. Quarz ist akzessorisch (um $1 \%$ ), vorwiegend in gerundeter Form als Calcit-Kristalleinschluss, am Gesteinsaufbau beteiligt. Weiterhin sind vereinzelt Bahnen von Flüssigkeitseinschlüssen zu beobachten.

Eine bevorzugte Regelung der Korngrenzen ist für die verwitterte Probe nur in der YZ-Ebene diagonal zur Y-Richtung und somit auch zur Foliation festzustellen (Abb. 4.12c). Die Textur der beiden Sterzinger Proben zeigt das Muster eines c-Achsenfasertyps, wobei die Intensität der verwitterten Probe durch ein c-Achsenmaximum von 3,4 VGV gekennzeichnet ist (Abb. 4.12d). Damit ist der Sterzinger Marmor auch hinsichtlich seiner Textur vergleichbar mit dem Grosskunzendorfer Marmor.

Das KL-Inventar wird durch eine homogene bläuliche Lumineszenz geprägt (s. Tafel 3, Abb. 5).

\section{- Kauffunger Marmor (Korngefügetyp III)}

Der Kauffunger Marmor repräsentiert einen Calcit Marmor, der deutliche Anteile an Dolomit in versiegelten Rissen und Alterationsbahnen aufweist. Das Mikrogefüge ist im Wesentlichen durch die typischen Merkmale eines Protomylonits charakterisiert (Tafel 1, Abb. 6). Das Gestein zeichnet sich durch eine große Vielfalt an unterschiedlichsten Gefügeelementen aus, die eine komplexe geologische Entwicklungsgeschichte anzeigen.

Das Korngefüge dominieren ausgelängte Calcitkörner (Altkörner, mittlere Korngröße bis $500 \mu \mathrm{m}$ ), welche randlich von feinkörnigen, meist polygonalen Körnern umsäumt sind (Neukörner, Korngröße unter $40 \mu \mathrm{m}$ ). Die Altkörner weisen unregelmäßige Formen auf mit stark buchtigen, ineinandergreifenden Korngrenzen (vgl. Abb. 4.13a,b). Intern sind sie durch häufiges Auftreten von verbogenen Zwillingen gekennzeichnet. Die kleineren Neukörner besitzen dagegen keine Internstrukturen. Vereinzelt sind Übergänge zwischen Alt-, Sub- und Neukörnern $\mathrm{zu}$ beobachten, die auf eine Rotationsrekristallisation hindeuten (s. Tafel 2, Abb. 6).

Eine strenge Formregelung der Altkörner liegt parallel zur Foliation. Dies ist durch eine ausgeprägte Korngrenzenregelung parallel zur Y-Richtung in der YZ-Ebene und parallel zur $\mathrm{X}$-Richtung in der XZ-Ebene nachgezeichnet (Abb. 4.13c). Eine etwas schwächere Regelung ist in der XY-Ebene parallel zur Y-Richtung festzustellen, womit die bevorzugte Kornform ein dreidimensionales Ellipsoid darstellt. Die Textur der verwitterten wie auch der frischen Probe des Kauffunger Marmors ist vergleichbar ausgebildet und entspricht dem Muster des cAchsenfasertyps. Die Proben zeigen mittlere bis starke Intensität der Textur, wobei das verwitterte Material ein c-Achsenmaximum mit 2,7 VGV aufweist (Abb. 4.13d).

Das auffälligste Merkmal des Mikrogefüges ist das häufige Auftreten von Mikrorissen. Mikroskopisch können zwei Gruppen von präexistierenden Mikrorissen unterschieden werden, welche ein kataklastisches Stadium des Gesteins repräsentieren (vgl. RUEDRICH et al., 2001b). Dabei handelt es sich um einen versiegelten und einen mineralisierten Risstyp. 


\section{Sterzing}

\section{Korngefüge}

- seriate interlobat

Mittlere Korngröße

- ca. $470 \mu \mathrm{m}$

Korngrenzengeometrie

buchtig bis verzahnt

\section{Korngrenzenregelung}

- deutliche Regelung in der YZEbene schräg zur Foliation

Textur

c-Achsenfasertyp starker Intensität

\section{Korngefüge}

a)
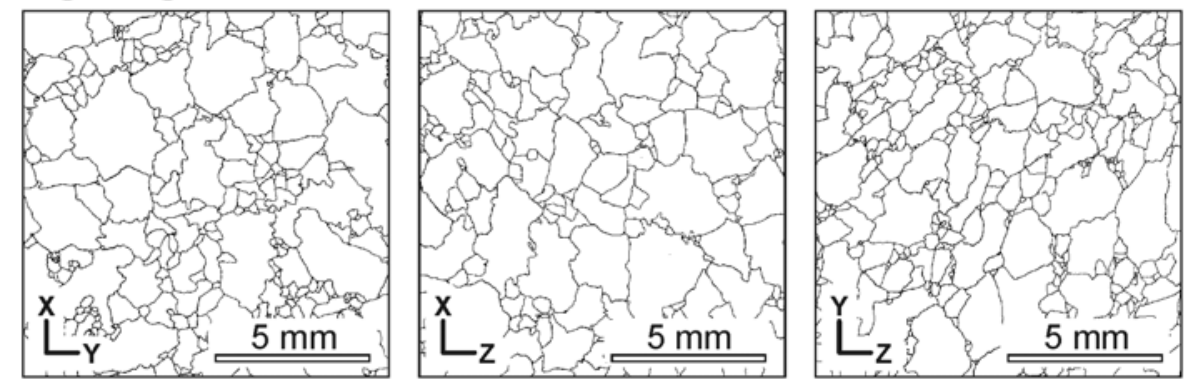

Kornflächenverteilung
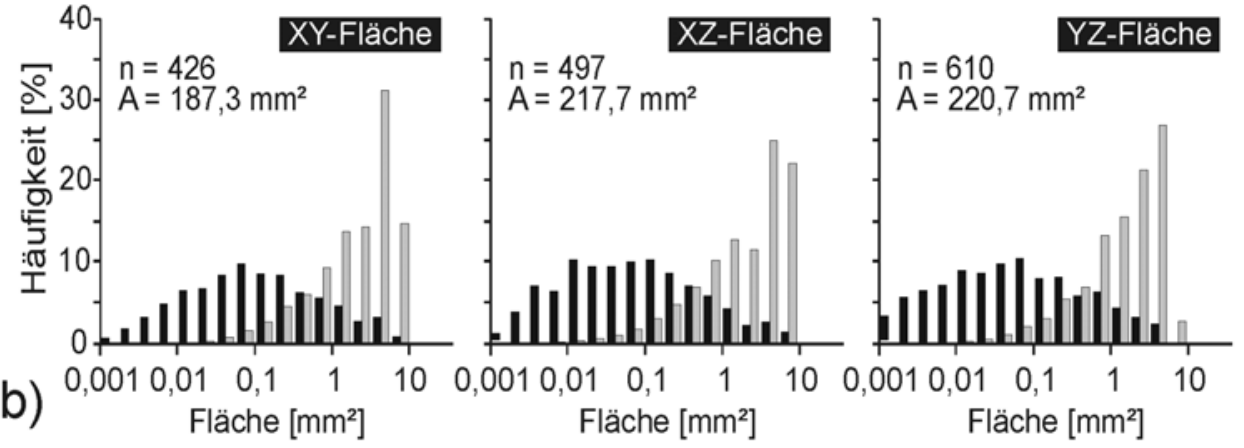

Korngrenzenregelung

c)

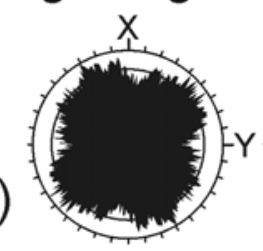

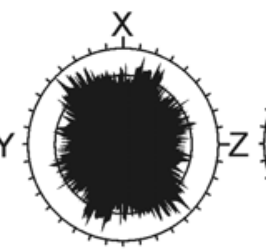

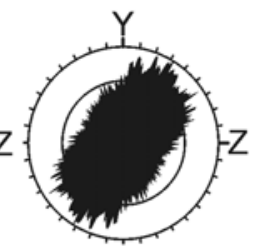

Textur

d)
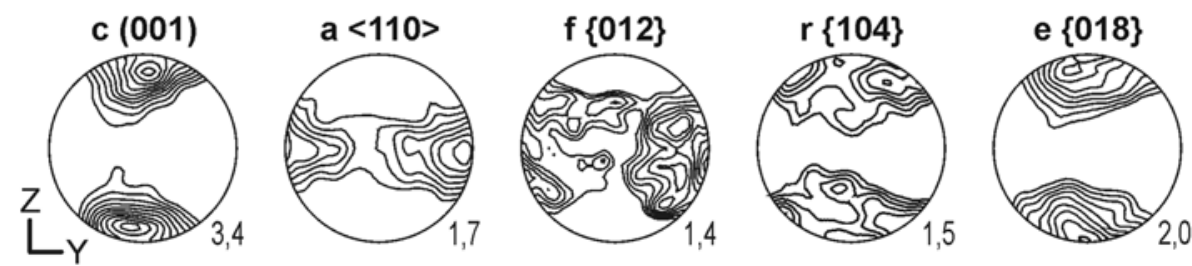

Abb. 4.12: Wesentliche Gefügemerkmale der drei orthogonalen Schnittlagen der verwitterten Probe aus Sterzing Marmor: a) Korngefüge, b) Korngrößenverteilung, dargestellt als Flächenparameter ( $\mathrm{n}=$ Anzahl und $\mathrm{A}=$ Gesamtfläche der analysierten Körner), $\mathrm{c}$ ) bevorzugte Orientierung der Korngrenzen und d) Textur (Intensität in VGV = Vielfaches der Gleichverteilung). 
Der versiegelte Typ weist eine Füllung durch überwiegend Calcit und untergeordnet Dolomit auf. Daneben sind auch feinverteilte Fe-Oxide als Mineralisate an der Füllung beteiligt. Die Körnung der beiden Hauptphasen kann sowohl feinkörniger als auch grobkörniger als das Hauptgestein sein (vgl. RichTER, 2000), wobei letztere Ausbildungsform dominiert. Die Calcit-Kristalle innerhalb der versiegelten Risse des Kauffunger Marmors weisen z.T. einen deutlichen KL-Farbzonarbau auf. Dieses weist auf chemische Variationen der Zusammensetzung der beteiligten fluiden Phase bzw. wechselnde Temperatur/Druckbedingungen während der Kristallisation der Calcite hin. Der Wechsel von hellgelber und dunkelbrauner Lumineszenz in den einzelnen Zonen ist hier auf den variierenden Einbau von $\mathrm{Mn}^{2+}$ Kationen als Spurenelement zurückzuführen (vgl. REEDER, 1991). Der verheilte Risstyp ist durch die typische syntaxiale Anwachsung des Calcits gekennzeichnet. Dieser Typ ist nur mittels KL-Mikroskopie anhand seiner leuchtend gelben KL-Farbe von der dunkelbraun bis dunkelblau lumineszierenden Matrix zu unterscheiden (s. Tafel 3, Abb. 6).

Ein weiteres auffälliges Gefügemerkmal des Kauffunger Marmors ist die starke Alteration des Gesteins, die lokal zur Sprossung von Sattel-Dolomit führt (vgl. SKOwroneK, 2001). Nach KL-Untersuchungen befinden sich die Sprossungen im Zentrum der alterierten Bereiche, die durch zunehmend intensiver werdende Gelbnuancen des Calcits gekennzeichnet sind.

Für weitere Informationen sowie Details zu gefügekundlichen Merkmalen und zur Heterogenität siehe auch Siegesmund et al. (1997), SKowroneK \& StefFAhn (2000), SKOWRONEK (2001), Richter (2001) und RUEDRICH et al. (2001b).

\subsubsection{Verwitterungsgefüge (offene Mikrorisse)}

Marmore stellen im unverwitterten Zustand kompakte kristalline Gesteine dar. Sie beinhalten nur lokal offene Mikrorisse, welche z.B. auf Relaxationsprozesse des Gesteinskörpers während der Exhumierung zurückzuführen sind. Der Großteil der geöffneten Risse wird hingegen durch die Gewinnung und die Verarbeitung des Materials sowie vor allem durch die Verwitterung am Bauwerk erzeugt. Die zunehmende Anlage von Rissen geht einher mit der fortschreitenden Entfestigung der Marmore. Geöffnete Mikrorisse bilden dabei den eigentlichen Porenraum und werden daher von vielen Autoren auch als „Plattenspaltporen“ bezeichnet (vgl. POSCHLOD, 1989). Die folgenden Betrachtungen beziehen sich auf die verwitterten Proben.

Für die untersuchten Marmore sind signifikante Unterschiede in der Art sowie der Verteilung der Mikrorisse und damit auch der Anordnung des Porenraums festzustellen. So kann für die meisten Marmore eine penetrativ das Gestein durchdringende, relativ gleichmäßige Rissbildung beobachtet werden. Demgegenüber steht das Auftreten mehr lokaler, isolierter Risse vor allem im Kauffunger Marmor. Daneben können die Mikrorisse intergranular (Korngrenzenrisse) und intragranular (Spalt- und Zwillingsflächen) angelegt sein. Bezüglich der Art der Risse sind Übereinstimmungen zwischen Rissmuster und den oben definierten unterschiedlichen Korngefügetypen festzustellen. Resultierend können drei verschiedene Risstypen definiert werden (Abb. 4.14). 


\section{Kauffung}

\section{Korngefüge}

seriate polygonal bis interlobat

Mittlere Korngröße

- ca. $40 \mu \mathrm{m}$

Korngrenzengeometrie

- buchtig bis verzahnt (Altkörner) gerade bis geschwungen (Subkörner)

\section{Korngrenzenregelung}

- parallel zur Foliation und in der XY-Ebene parallel zur Y-Richtung

\section{Textur}

c-Achsenfasertyp mittlerer

\section{Korngefüge}

a)
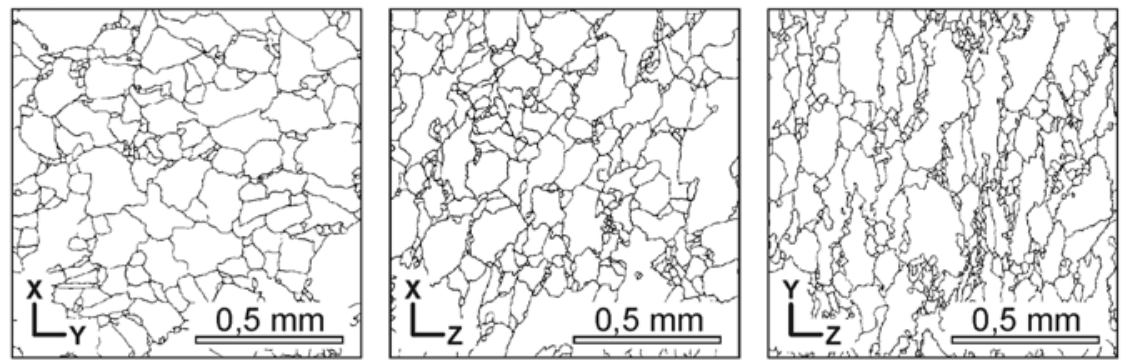

Kornflächenverteilung
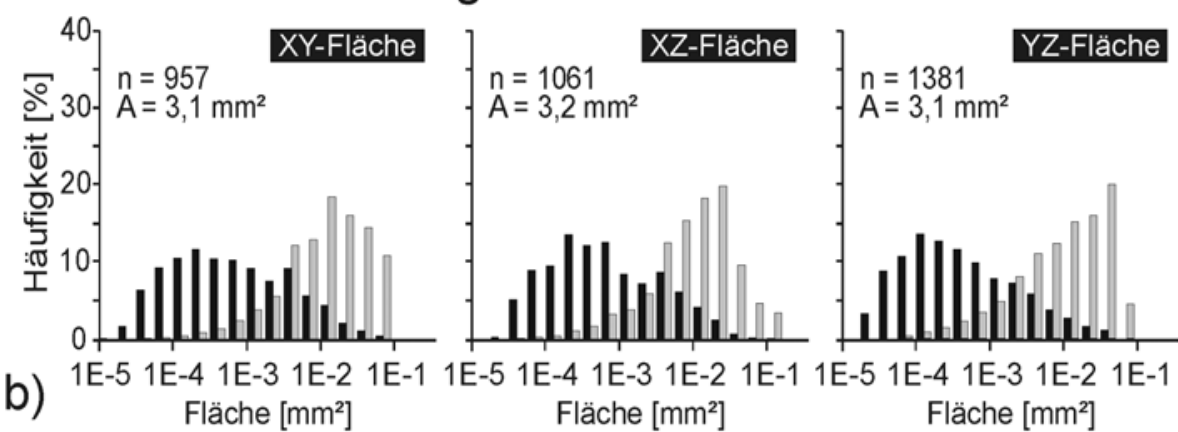

Korngrenzenregelung

c)

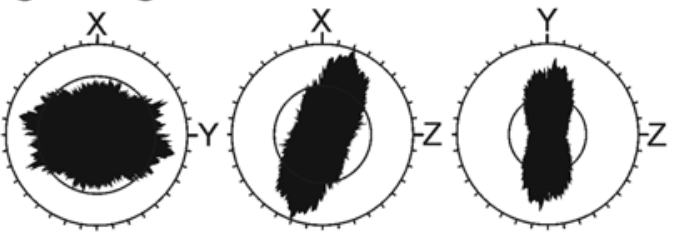

Textur

d)
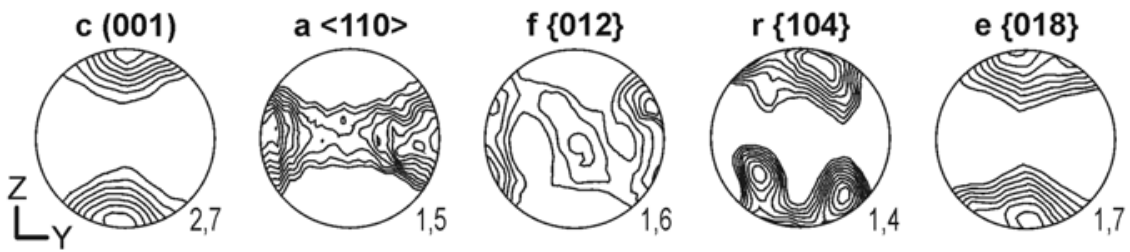

Abb. 4.13: Wesentliche Gefügemerkmale der drei orthogonalen Schnittlagen der verwitterten Probe aus Kauffung Marmor: a) Korngefüge, b) Korngrößenverteilung, dargestellt als Flächenparameter ( $\mathrm{n}=$ Anzahl und $\mathrm{A}=$ Gesamtfläche der analysierten Körner), c) bevorzugte Orientierung der Korngrenzen und d) Textur (Intensität in VGV $=$ Vielfaches der Gleichverteilung). 
Der Risstyp I kennzeichnet eine penetrative Mikrorissbildung fast ausschließlich an Korngrenzen (Abb. 4.14a; Tafel 4, Abb. 1). Diese Rissentfestigung kann für die equigranular polygonalen Marmore aus Carrara, Prieborn und Lasa (Korngefügetyp I) festgestellt werden (vgl. Tafel 5, Abb. 1-3).

Im Gegensatz dazu ist der Risstyp II bei den seriaten interlobaten Marmoren aus Sterzing und Grosskunzendorf des Korngefügetyp II festzustellen. Hierbei dominieren neben Korngrenzenrissen vor allem auch intragranulare Risse entlang von Spaltflächen und Zwillingsebenen (Tafel 5, Abb. 4-5). Die Risse erzeugen dabei ebenfalls eine durchdringende Entfestigung des Korngefüges (Abb. 4.14b; Tafel 4, Abb. 2-5).

Risstyp III wird durch den Kauffunger Marmor (Korngefügetyp II) repräsentiert, der auch bezüglich der Rissentfestigung bei den vorliegenden Untersuchungen eine Sonderstellung einnimmt (vgl. Tafel 4, Abb. 6; Tafel 5, Abb. 6). Hier werden weitgehend mineralisierte präexistierende Risse reaktiviert. Dabei verlaufen die Risse zumeist an der Grenze zwischen präexistierendem Riss und Hauptgestein (Abb. 4.14c). Vereinzelt ist auch ein Ausweichen der geöffneten Risse in das Muttergestein zu beobachten in Form typischer transgranularer Risse. Nur lokal können auch intragranulare Risse bevorzugt in großen reliktischen Altkörnern festgestellt werden. Sie bilden jedoch keine penetrative Rissentfestigung des Gesteins, wie sie für die beiden anderen Risstypen beobachtbar sind.

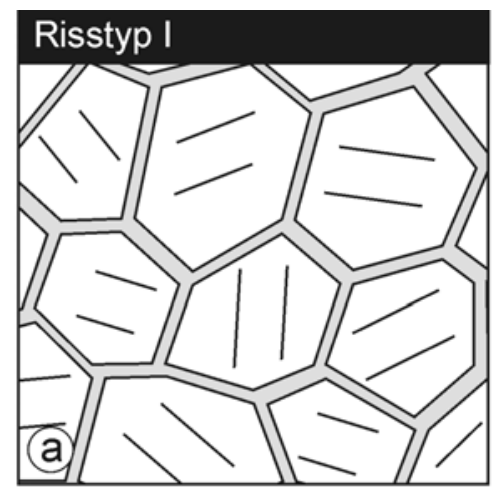

intergranulare Risse gesteinsdurchdringend

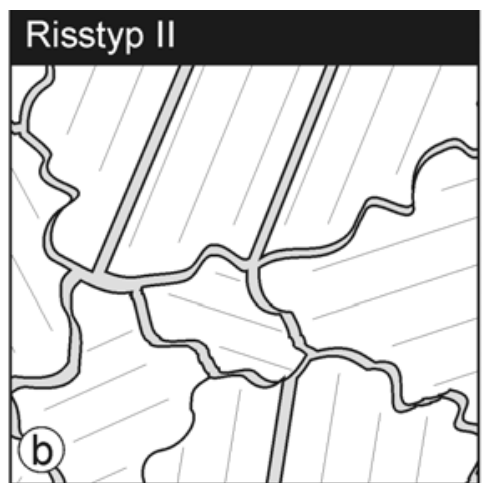

inter- und intragranulare Risse gesteinsdurchdringend

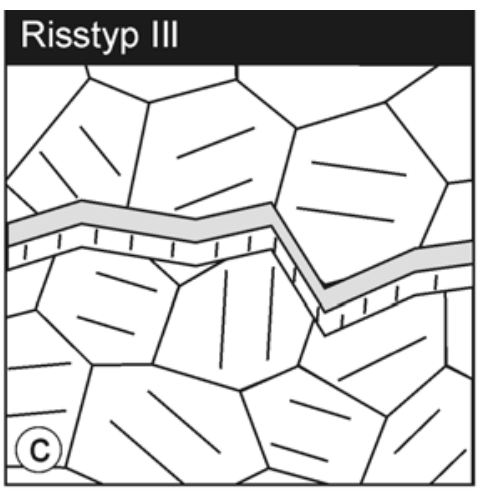

lokale Reaktivierung präexistierender Risse

Abb. 4.14: Verteilungsmuster von Mikrorissen in Abhängigkeit vom Korngefügetyp: a) penetrativ angelegte Mikrorisse entlang von Korngrenzen (Korngefügetyp I), b) inter- sowie intragranulare Mikrorisse (Korngefügetyp II) und c) lokale Mikrorissentfestigung entlang eines präexistierenden Risses (Korngefügetyp III).

\section{- Lösungsgefüge}

Für die untersuchten verwitterten Marmore kann eine Modifikation der Rissgeometrien durch Lösungsgefüge festgestellt werden. Diese treten häufig bei den stark verwitterten Marmoren aus Carrara und Grosskunzendorf auf. Die Lösungsgefüge sind dabei offensichtlich an energetisch begünstigte Stellen der Kristalloberflächen gebunden, welche Dislokationen, Punktdefekte und Zwillingsebenen sowie aktivierte Spaltflächen sein können (vgl. auch PiELOW, 1997). 
Als Lösungsgefüge können Ätzgrübchen auf Kristalloberflächen und Lösungskanäle beobachtet werden (Tafel 6, Abb. 1-6). Die Geometrie der Ätzgrübchen kann sehr unterschiedlich sein. Es kommen sowohl rund erscheinende als auch eckige, zumeist länglich dreieckige Hohlstellen vor. Lösungskanäle sind entlang von Spaltflächen und vor allem Zwillingsebenen der Kristalle lokalisiert. Die Intensität der Lösung kann dabei extrem stark sein, was zu tiefen spaltenartigen Hohlstellen führt. Ätzgrübchen können vor allem bei den equigranularen polygonalen Marmoren des Korngefügetyps I beobachtet werden, wohingegen der Grosskunzendorfer Marmor als Vertreter des Korngefügetyps II bevorzugt Lösungskanäle aufweist. 


\section{Tafel 1: Korngefüge}
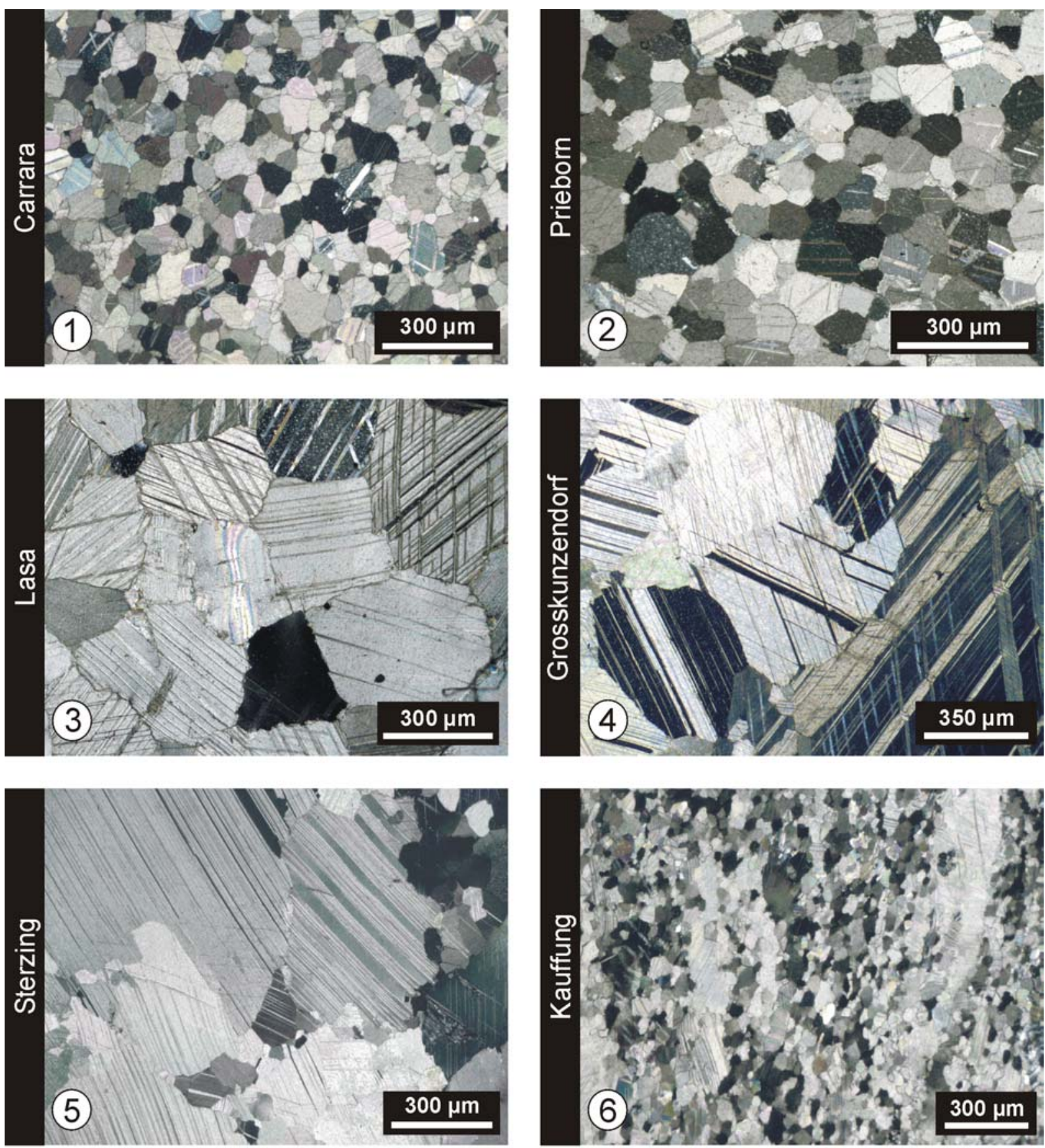

Abb. 1: Equigranulares Korngefüge des feinkörnigen Carrara Marmors mit polygonalen Kornformen (POL, YZ-Ebene, lange Bildkante \| Z-Richtung).

Abb. 2: Gleichkörnig polygonales Korngefüge des feinkörnigen Prieborner Marmors (POL, YZEbene, lange Bildkante $\|$ Z-Richtung).

Abb. 3: Equigranular polygonales Korngefüge des kleinkörnigen Lasa Marmors mit häufigem Auftreten von Zwillingen (POL, YZ-Ebene, lange Bildkante || Z-Richtung).

Abb. 4: Seriates Korngefüge des Grosskunzendorfer Marmors mit ausgeprägter Kornverzahnung und häufiger Ausbildung von Zwillingen (POL, YZ-Ebene, lange Bildkante || Z-Richtung).

Abb. 5: Seriates interlobates Korngefüge des Sterzinger Marmors mit vereinzelter Subkornbildung und häufigem Auftreten von Zwillingen (POL, YZ-Ebene, lange Bildkante || Z-Richtung).

Abb. 6: Durch Auflösung von länglichen Altkörnern durch Sub- und Neukornbildung geprägtes Korngefüge des Kauffunger Marmors (YZ-Ebene, lange Bildkante \|| Z-Richtung). 


\section{Tafel 2: Korngrenzengefüge}
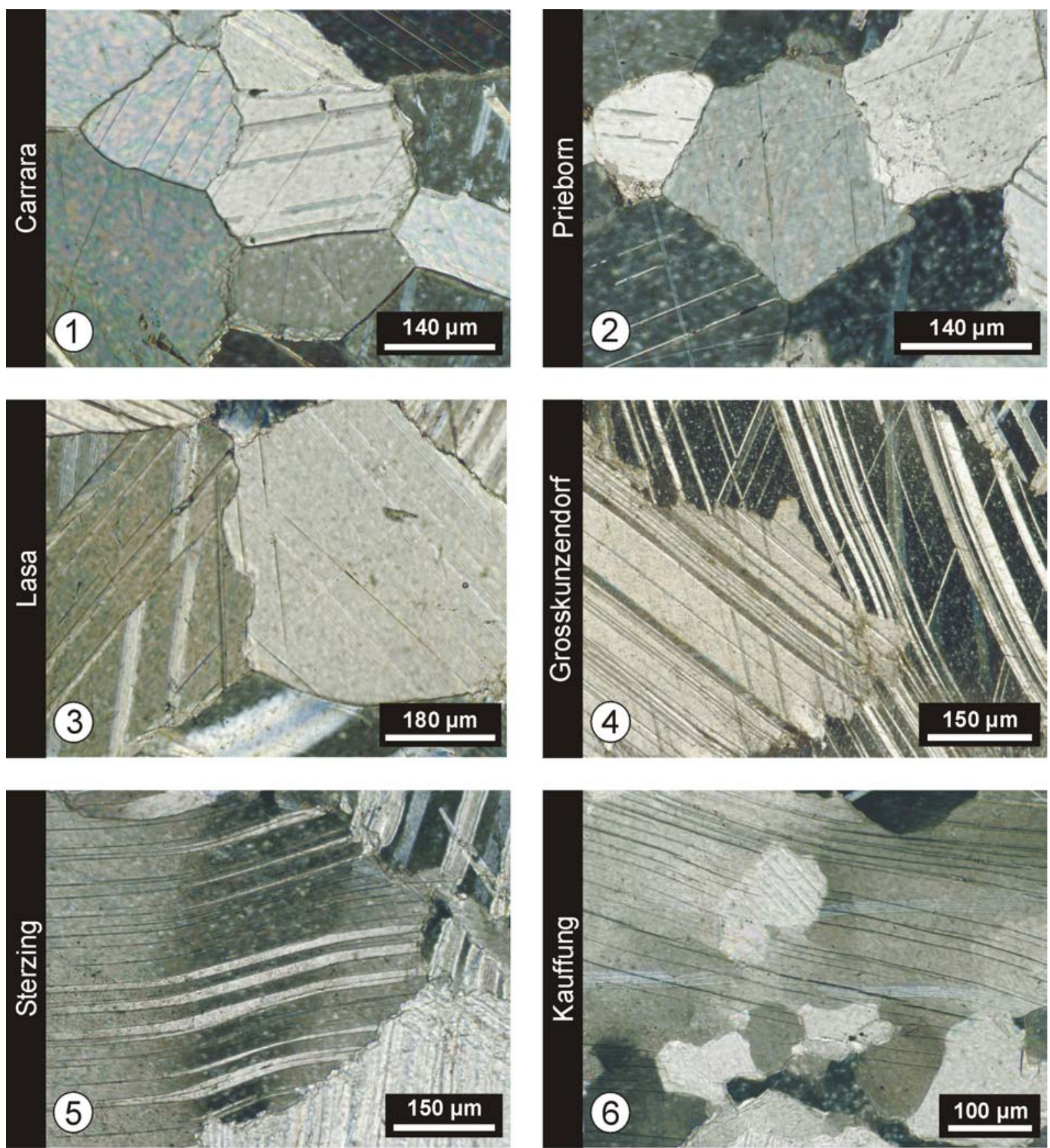

Abb. 1: Equilibriertes Korngefüge des Carrara Marmors mit geraden bis leicht geschwungenen Korngrenzengeometrien (pflasterartiges Gefüge; POL)

Abb. 2: Geschwungene bis leicht gezahnte Korngrenzengeometrien des Prieborner Marmors (POL).

Abb. 3: Geschwungen bis leicht buchtige Korngrenzengeometrien des Lasa Marmors (POL).

Abb. 4: Buchtig bis verzahnte Korngrenzengeometrien von ineinandergreifenden Körnern des Grosskunzendorfer Marmors (POL).

Abb. 5: Stark buchtige bis schwach gezackte Korngrenzen von unregelmäßig verzahnten Körnern des Sterzinger Marmors (POL).

Abb. 6: Reliktisches Altkorn mit Subkorn und Neukornbildung des Kauffunger Marmors (POL). 


\section{Tafel 3: KL-Eigenschaften}
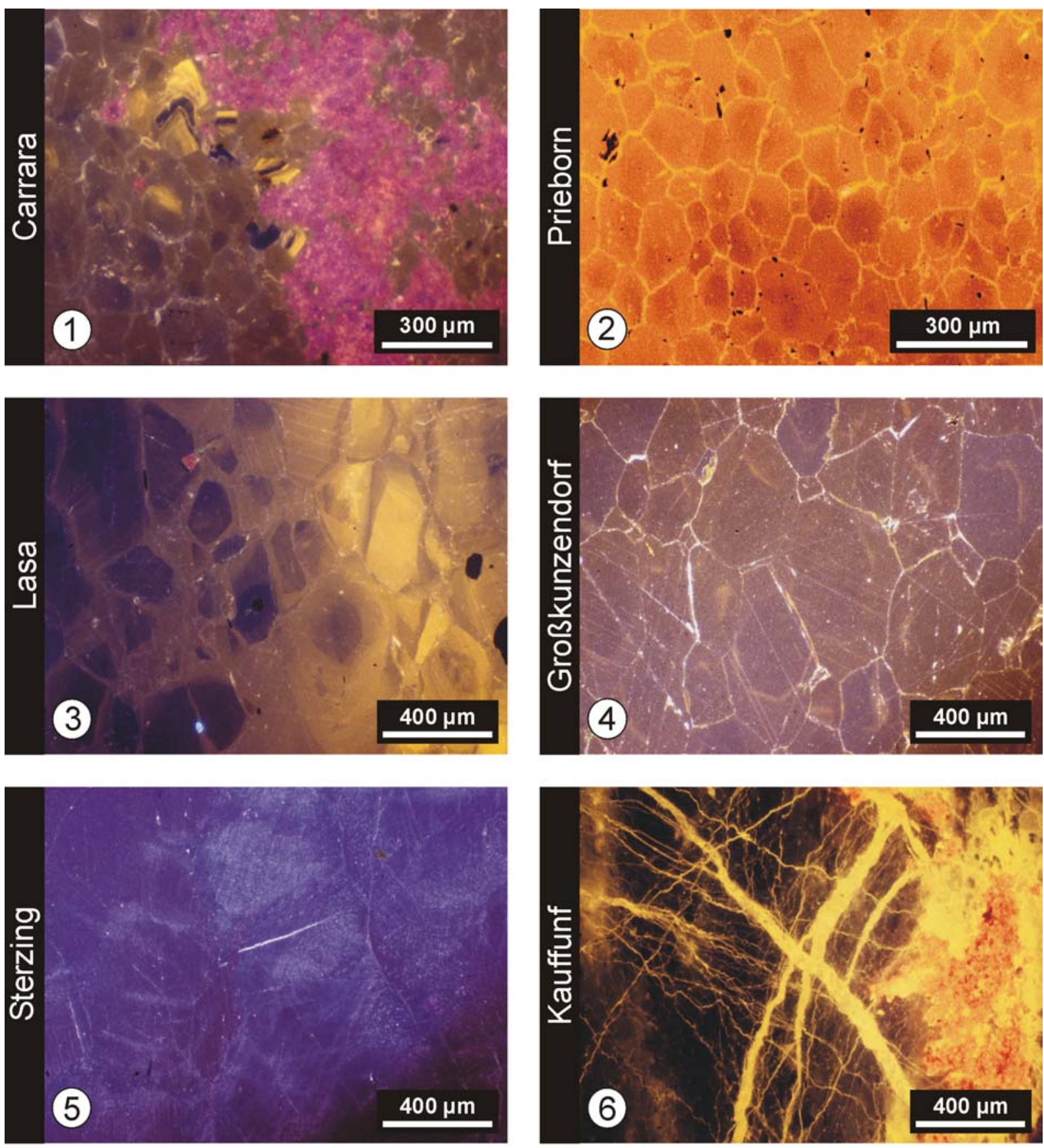

Abb. 1: Braun lumineszierende Calcit-Matrix und pinkfarbend lumineszierende Bereiche aus feinkörnigem Dolomit des Carrara Marmors. Zonierte Partien können als Relikte schalenbildender Organismen interpretiert werden (KLM).

Abb. 2: Orange lumineszierende Calcit-Körner des Prieborner Marmors mit ausgeprägter Korngrenzenalterationen, die an gelben Lumineszenzsäumen erkennbar ist (KLM).

Abb. 3: Durch einen Zonarbau von gelben und blauen Lumineszenzfarben gekennzeichnete CalcitKörner des Lasa Marmors. Fehlende Lumineszenz charakterisiert Quarz-Kristalle (KLM).

Abb. 4: Rötlich braun lumineszierende Calcit-Körner des Grosskunzendorfer Marmors (KLM).

Abb. 5: Weitgehend homogen violett lumineszierende Calcit-Körner des Sterzinger Marmors (KLM).

Abb. 6: Braun lumineszierende Matrix des Kauffunger Marmors sowie mineralisierte und versiegelte Mikrorisse mit gelber Lumineszenz. Rot lumineszierende Bereiche repräsentieren sekundär gesprosste Dolomit Kristalle (KLM). 


\section{Tafel 4: Mikrorisse}
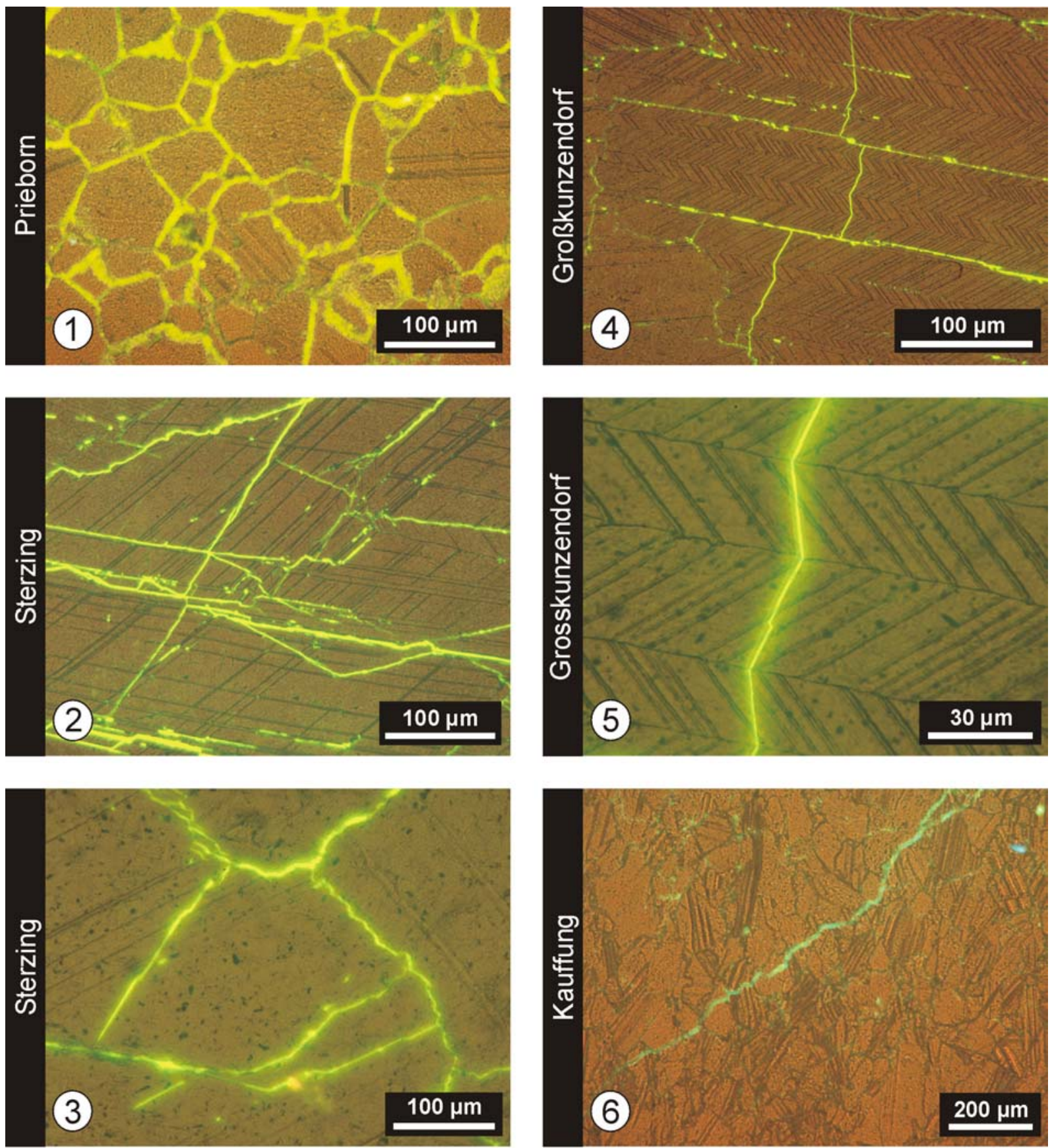

Abb. 1: Vollständige Korngrenzenentfestigung des Carrara Marmors, die durch Besetzung der intergranularen Risse durch fluoreszierendes Harz erkennbar ist (Korngefügetyp I; FLM).

Abb. 2: Komplex entwickeltes intragranulares Mikrorissnetzwerk in einem Korn des Sterzinger Marmors (Korngefügetyp II; FLM).

Abb. 3: Mikrorisse entlang überwiegend intergranularer Flächen im Sterzinger Marmor (Korngefügetyp II; FLM).

Abb. 4: Inter- und intragranulare Rissentfestigung eines Kornes im Grosskunzendorfer Marmor (Korngefügetyp II; FLM).

Abb. 5: Isolierter intragranularer Mikroriss diagonal zu Spaltflächen und Zwillingsflächen (Teilausschnitt aus Abb. 4, Grosskunzendorfer Marmor; FLM).

Abb. 6: Isolierter transgranularer Mikroriss im Kauffunger Marmor. Die Matrix zeigt hingegen nur sehr vereinzelt lokale Rissbildung (Korngefügetyp III; FLM). 


\section{Tafel 5: Bruchstrukturen}
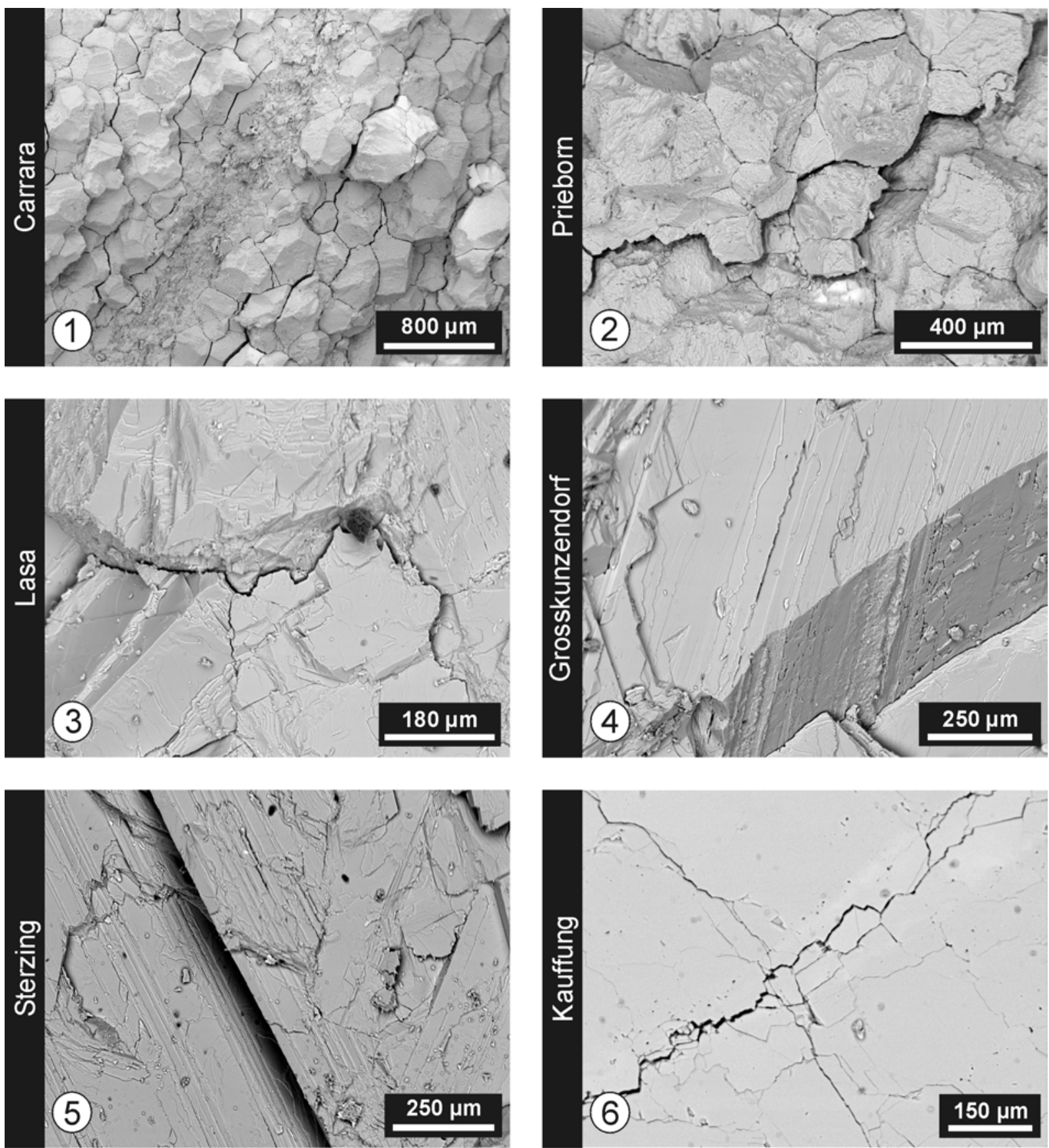

Abb. 1: Verwitterte Probe aus Carrara Marmor die eine vollständige Entfestigung an Korngrenzen aufweist. Im Zentrum befindet sich ein Band sehr feinkörniger Verwachsungen von Dolomit und Calcit (REM, Fraktographie).

Abb. 2: Intergranulare Risse der verwitterten Probe aus Prieborner Marmor (REM, Fraktographie).

Abb. 3: Entfestigte Korngrenzen des Lasa Marmors. Durch das Brechen der schwach verwitterten Probe wurden verbreitet auch intragranulare Flächen aktiviert (REM, Fraktographie).

Abb. 4: Vollständig intragranulare Rissbildung einer Bruchprobe des Grosskunzendorfer Marmors (REM, Fraktographie).

Abb. 5: Intragranulare Rissbildung einer Bruchprobe des Sterzinger Marmors (REM, Fraktographie).

Abb. 6: Senkrecht aufeinanderstehendes Mikrorisssystem im Kauffunger Marmor (REM, polierter Dünnschliff). 


\section{Tafel 6: Lösungsgefüge}
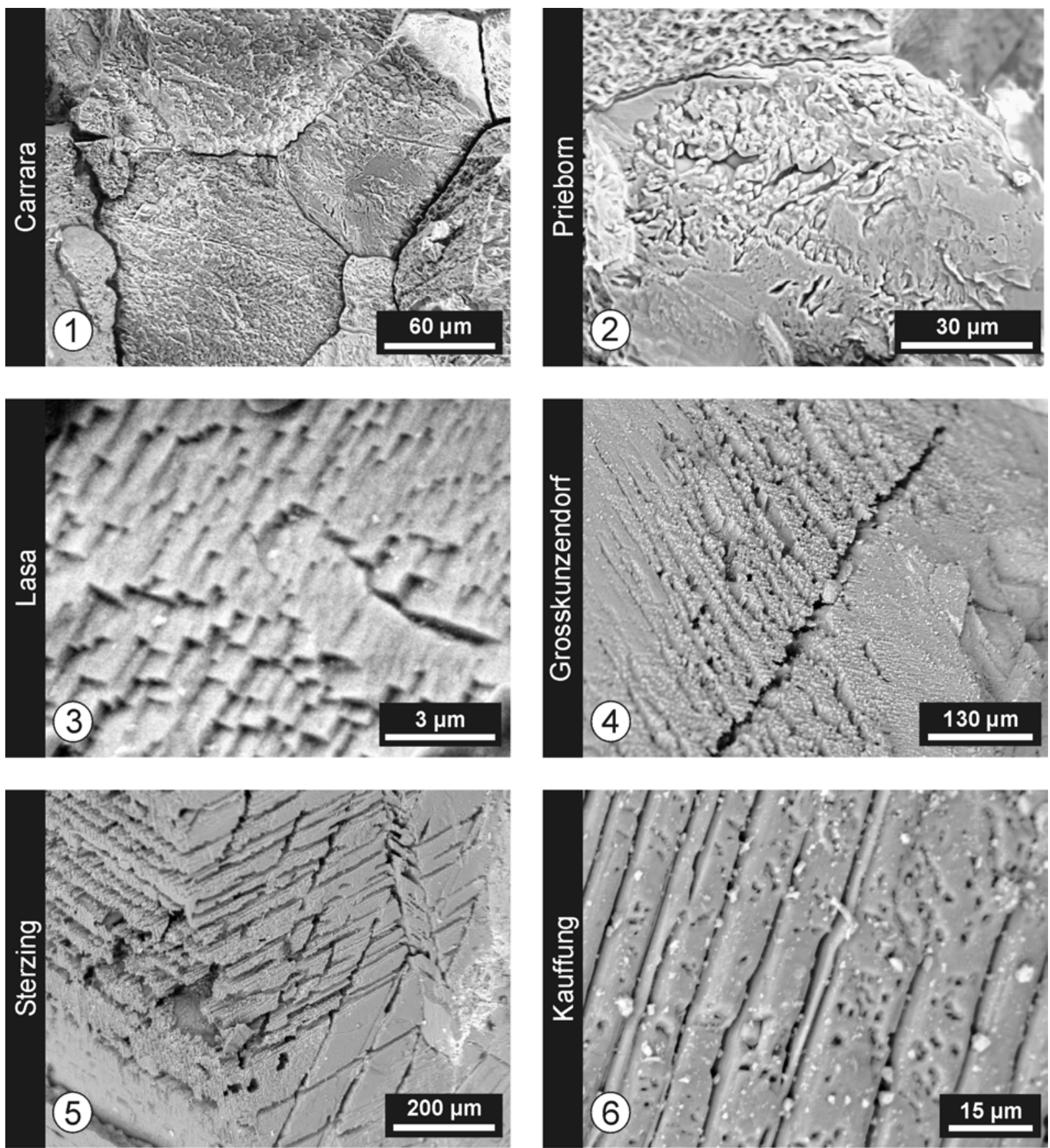

Abb. 1: Durch Lösungsgefüge erzeugte starke Reliefbildung auf Korngrenzen des Carrara Marmors (REM, Fraktographie).

Abb. 2: Unregelmäßige Lösungserscheinungen auf Korngrenzen des Carrara Marmors (REM, Fraktographie).

Abb. 3: Symetrische Lösungsgefüge auf einer Korngrenzenfläche des Carrara Marmors (REM, Fraktographie).

Abb. 4: Von einem zentralen Mikroriss ausgehende, unregelmäßige spaltenartige Lösungsgefüge entlang intragranularer Flächen im Grosskunzendorfer Marmor (REM, Fraktographie).

Abb. 5: Tiefe Hohlraumbildungen durch chemische Lösung entlang intragranularer Schwachstellen des Grosskunzendorfer Marmors (REM, Fraktographie).

Abb. 6: Lösungsphänomene entlang von intragranularen Flächen mit Ätzgrübchen auf den dazwischen liegenden Stegen des Grosskunzendorfer Marmors (REM, Fraktographie). 


\subsection{Petrophysikalische Eigenschaften}

Voraussetzung für eine Interpretation der Entfestigungsprozesse von Marmoren ist eine Charakterisierung der verwitterungsbedingten Veränderung von Gesteinseigenschaften. Dazu erfolgten in der vorliegenden Arbeit vergleichende Analysen verwitterungssensibler petrophysikalischer Parameter an den ausgewählten unverwitterten und im unterschiedlichen Maße verwitterten Marmoren. Zur Charakterisierung der entfestigungsbedingten Veränderung des Porenraums wurden Messungen der Porosität und der Porengrößenverteilungen durchgeführt. Zusätzlich wird die Vernetzung der Porenräume mittels der kapillaren Wasseraufnahme beschrieben. Die Erfassung der elastischen Eigenschaften erfolgte mittels Ultraschallanalysen und dient der quantitativen Charakterisierung der Mikrorisssysteme. Die Ultraschallanalytik wurde unter dem Gesichtspunkt ihrer Eignung für die Diagnostik des Erhaltungszustandes von Marmoren durchgeführt. Ferner erfolgte die richtungsabhängige Charakterisierung der Kohäsion des Gefügeverbundes mittels Messungen der Spaltzugfestigkeit. Für die Ultraschall- und Festigkeitseigenschaften stand im Vordergrund, in welcher Art und in welchem Umfang ihre Veränderungen während der Verwitterung materialspezifisch sind und auf unterschiedliche Gefügeparameter wie Korngefüge, Textur etc. zurückzuführen sind. Berücksichtigung fand dabei der Einfluss der in Marmoren weit verbreiteten Gefügeanisotropien auf die richtungsabhängige Gesteinsentfestigung.

\subsubsection{Porenraumeigenschaften}

Die Verwitterung von Marmoren führt zu einer deutlichen Veränderung des Porenraums welche über die Porosität und die Porengrößenverteilung beschrieben werden kann. Dabei besitzt die Porosität besondere Bedeutung für das mechanisch physikalische Gesteinsverhalten, z.B. elastische Eigenschaften oder Wärmeleitfähigkeit. Die Porengrößenverteilung kontrolliert vor allem die Wassertransportmechanismen und ist damit für Verwitterungsmechanismen, wie z.B. chemische Lösungsprozesse von Interesse.

\section{- Porosität}

Die Porosität kristalliner Marmore ist sowohl im unverwitterten als auch im verwitterten Zustand relativ gering. Marmore weisen im frischen Zustand Porositäten um 0,2 \% auf (vgl. POSCHLOD, 1989). Für stark verwitterte Marmore sind hingegen Porositäten bis annähernd $3 \%$ festzustellen (vgl. WEISS et al., 2000), wobei von einigen Autoren auch Porositäten von bis zu $5 \%$ ermittelt wurden (HOLTKAMP, 1999).

Für die untersuchten Marmorproben liegen die im unverwitterten Zustand mittels Auftriebswägung gemessenen effektiven Porositäten $(\Phi)$ zwischen ca. $\Phi=0,2 \%$ und $\Phi=0,4 \%$ (Tab. 4.1). Damit besitzen diese Gesteine zwar einen sehr kleinen Porenraum, der jedoch anzeigt, dass bereits vernetzte Risse im Gestein vorhanden sind. Entsprechende Porositäten können schon in der Lagerstätte entstehen, z.B. durch Exhumierung des Gesteinskörpers in Verbindung mit Druckentlastung (vgl. VollbRECHT et al., 1991; SCHILD et al., 2001). Aber auch durch die Gewinnung (Herauslösung aus dem Gesteinsverband) sowie durch die Präparation können entsprechende Porositäten erzeugt werden. 
Tab. 4.1: Effektive Porositäten (mittlere Werte) ermittelt durch Auftriebswägung und Quecksilberporosimetrie sowie häufigst besetzte Porenradienklasse (mittlerer Wert) der untersuchten unverwitterten und verwitterten Marmore (fett $=$ über $0,1 \mu \mathrm{m}$ ).

\begin{tabular}{|c|c|c|c|c|}
\hline \multirow[b]{2}{*}{ Marmor Typ } & \multirow[b]{2}{*}{ Zustand } & \multicolumn{2}{|c|}{ Porosität [Vol.-\%] } & \multirow{2}{*}{$\begin{array}{c}\text { häufigst besetzte } \\
\text { Porenradienklasse } \\
{[\mu \mathrm{m}]}\end{array}$} \\
\hline & & $\begin{array}{l}\text { Auftriebs- } \\
\text { wägung }\end{array}$ & $\begin{array}{c}\mathrm{Hg}- \\
\text { Porosimetrie }\end{array}$ & \\
\hline \multirow{3}{*}{ Carrara } & unverwittert & 0,31 & 0,51 & 0,075 \\
\hline & verwittert & 0,50 & 0,92 & 0,422 \\
\hline & stark verwittert & 2,20 & 2,02 & 2,371 \\
\hline \multirow{2}{*}{ Prieborn } & unverwittert & 0,35 & 0,40 & 0,075 \\
\hline & verwittert & 0,55 & 0,96 & 0,237 \\
\hline \multirow{2}{*}{ Kauffung } & unverwittert & 0,20 & 0,37 & 0,042 \\
\hline & verwittert & 0,46 & 0,62 & 0,422 \\
\hline \multirow{2}{*}{ Grosskunzendorf } & unverwittert & 0,29 & 0,29 & 0,042 \\
\hline & verwittert & 0,74 & 0,85 & 0,422 \\
\hline \multirow{2}{*}{ Lasa } & unverwittert & 0,33 & 0,33 & 0,075 \\
\hline & verwittert & 0,44 & 0,60 & 0,237 \\
\hline \multirow{2}{*}{ Sterzing } & unverwittert & 0,30 & 0,20 & 0,237 \\
\hline & verwittert & 0,40 & 0,50 & 0,422 \\
\hline
\end{tabular}

Die effektiven Porositäten der verwitterten Marmore weisen eine Variation zwischen ca. $0,4 \%$ und 2,40 \% auf. In den meisten Fällen ist dabei nur eine geringfügige Erhöhung im Vergleich zum unverwitterten Zustand des jeweiligen Marmortyps festzustellen (s. Tab. 4.1). Deutlich höhere Porositätsunterschiede zeigt hingegen der Grosskunzendorfer Marmor mit ca. $\Delta \Phi=0,45 \%$ sowie die stark verwitterte Probe des Carrara Marmors, die im Vergleich mit $\Phi=2,20 \%$ fast das zehnfache der Porosität einer unverwitterten Probe aufweist.

In Tab. 4.1 sind zusätzlich die mittels Quecksilberporosimetrie ermittelten effektiven Porositäten für die entsprechenden Marmorproben aufgetragen. Im Vergleich $\mathrm{zu}$ den Ergebnissen der Auftriebswägung unterscheiden sich die Daten i.d.R. nur geringfügig. Die zumeist leicht erhöhten Werte der Quecksilberporosimetrie können auf Oberflächeneffekte und präparationsbedingte Entfestigungen zurückgeführt werden. Daneben sind die analysierten Zylinderproben relativ klein und damit nur bedingt repräsentativ für das Gesamtgestein.

\section{- Porengrößenverteilung}

Für Naturwerksteine ist die Porengrößenverteilung von besonderer Bedeutung, da das Verwitterungsverhalten eines Naturwerksteins signifikant von ihr beeinflusst werden kann (FitZNER, 1969; FitZNER \& SNETHLAGE, 1982). Für kristalline Marmore ist die Verteilung der Porengrößen für die Wassertransportmechanismen von besonderer Bedeutung. Die Porengrößenmaxima liegen dabei häufig im Bereich um $0,1 \mu \mathrm{m}$. Hier befindet sich der Übergang zwischen Wasserdampfdiffusion (unterhalb) und Kapillarwirkung (oberhalb) als wesentlicher Wassertransportmechanismus (SNethlage, 1984; KLOPFER, 1985; PosCHLOD, 1989). 
Der direkte Vergleich der Porengrößenverteilung von unverwitterten und verwitterten Marmoren soll anhand des Carrara und des Grosskunzendorfer Marmors dargestellt werden (s. Abb. 4.15). Beide unverwitterten Proben zeigen ein Porenradienmaximum im Bereich zwischen $0,032 \mu \mathrm{m}$ und $0,100 \mu \mathrm{m}$ (s. Abb. 4.15a,c). Damit liegen die Porengrößen überwiegend unter $0,100 \mu \mathrm{m}$, womit der wesentliche Wassertransportmechanismus die Wasserdampfdiffusion ist.
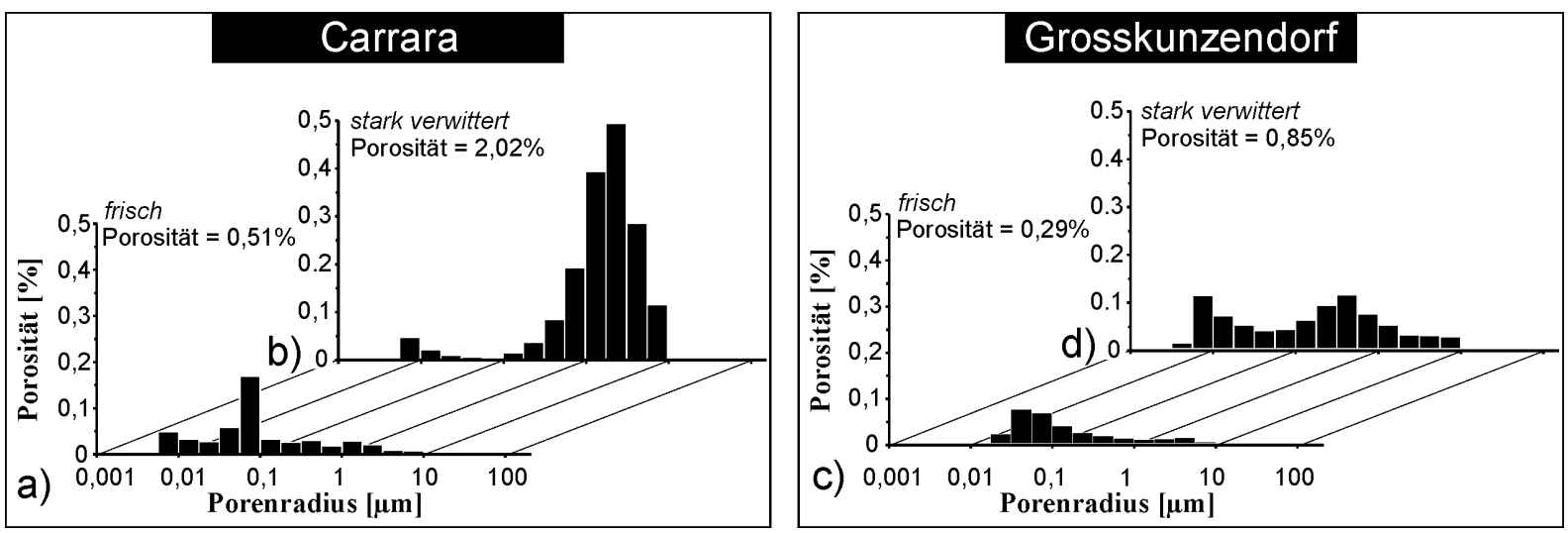

Abb. 4.15: Verteilung der Porenradien von a) frischem und b) stark verwittertem Carrara Marmor sowie c) frischem und d) stark verwittertem Grosskunzendorfer Marmor.

Die verwitterte Probe des Carrara Marmors weist ein signifikantes Porengrößenmaximum bei $0,560 \mu \mathrm{m}$ bis 5,600 $\mu \mathrm{m}$ auf, wobei sich ein Submaximum zwischen 0,006 $\mu \mathrm{m}$ und $0,018 \mu \mathrm{m}$ befindet (s. Abb. 4.15b). Der verwitterte Grosskunzendorfer Marmor ist hingegen durch eine bimodale Porenradienverteilung gekennzeichnet. Ein erstes Maximum liegt im Bereich von $0,006 \mu \mathrm{m}$ bis $0.032 \mu \mathrm{m}$, ein weiteres befindet sich zwischen $0,100 \mu \mathrm{m}$ und $1,000 \mu \mathrm{m}$ (s. Abb. 4.15d). Für die verwitterten Marmore sind damit generell Werte der häufigst besetzten Porenradienklasse oberhalb 0,100 $\mu \mathrm{m}$ festzustellen, womit diese Proben im Bereich des durch Kapillarkräfte gesteuerten Wassertransports liegen.

Die mittels Quecksilberporosimetrie gemessenen häufigst besetzten Porenradienklassen der ausgewählten Marmorproben sind in Tab. 4.1 aufgeführt. Die Daten zeigen auch für die weiteren Proben einen vergleichbaren Trend zu den o.a. Beispielen des Carrara und des Grosskunzendorfer Marmors. Eine Ausnahme bilden hier die unverwitterten Proben aus Prieborner und Sterzinger Marmor, wo sich die häufigst besetzte Porenradienklasse schon oberhalb der $0,100 \mu \mathrm{m}$ Grenze befindet.

Die ermittelten Daten zeigen, dass mit fortschreitender Verwitterung die Porosität zunimmt und die Porengrößen sich von kleinen zu großen entwickeln. Dabei stellt sich die Frage inwieweit diese beiden Parameter bei fortschreitender Verwitterung im Verhältnis zueinander stehen. Es ist davon auszugehen, dass für eine reine Rissweitung bei unveränderlicher Rissdichte ein gleichbleibendes Verhältnis zwischen Porositätszunahme und dem Zuwachs der Porengrößenverteilung existiert. Aufgrund der unterschiedlichen Häufigkeit von Rissen sollte dieses Verhältnis jedoch für jede Probe variieren.

Die Untersuchungen weisen daraufhin, dass sich bei stark verwitterten Proben dieses Verhältnis signifikant ändern kann. Dies ist Abb.4.16 für die verschiedenen 
Erhaltungszustände (unverwittert, verwittert und stark verwittert) dargestellt. Während für die unverwitterten und die verwitterten Proben ein starker Anstieg der Porengrößen und eine relativ geringe Zunahme der Porosität festzustellen ist, weisen die stark verwitterten Marmore eine deutlich erhöhte Porosität auf.

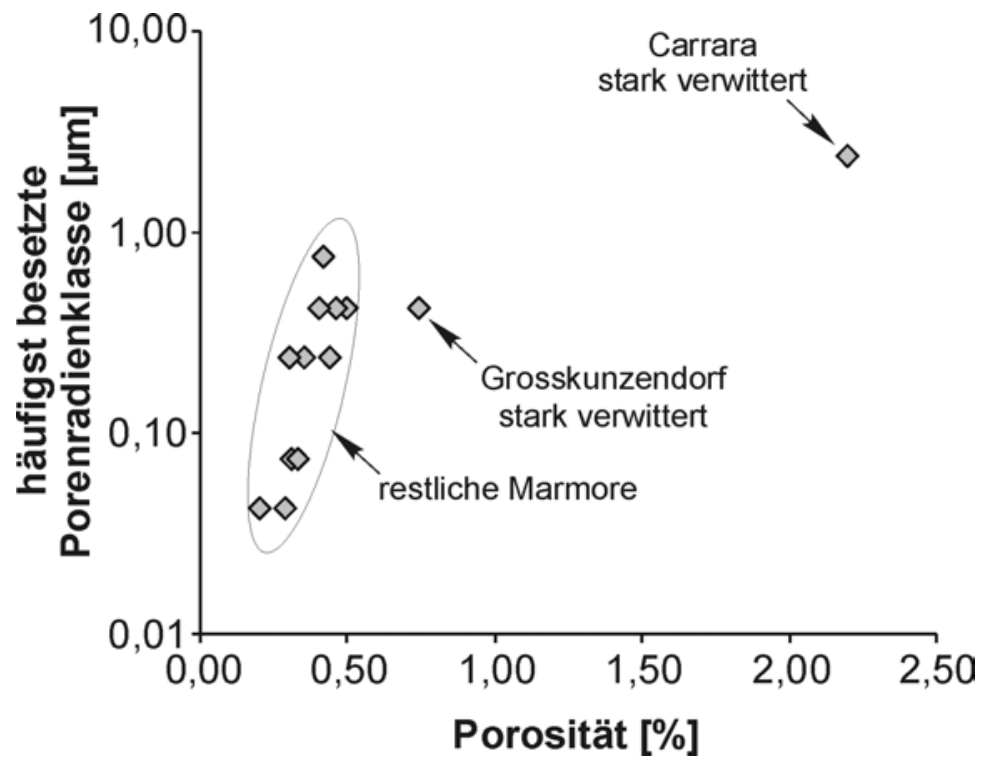

Abb. 4.16: Darstellung der häufigst besetzten Porenradienklasse (unterer Wert) als Funktion der Porosität.

Dieser verstärkte Anstieg der Porosität könnte mit einer Zunahme der Rissdichte erklärt werden. Die Bildung vieler kleiner Risse lässt die Porengrößenverteilung nicht oder nur gering ansteigen, führt aber $\mathrm{zu}$ einer deutlichen Porositätszunahme. Die Mikrobereichsanalysen zeigen jedoch, dass das Risssystem der stark verwitterten Marmore penetrativ ausgebildet und eine weitere Zunahme von Mikrorissen unwahrscheinlich ist. Die Rissgeometrie der besonders stark entfestigten Marmorproben ist nach mikrogefügekundlichen Untersuchungen durch eine Modifikation in Form von Lösungsgefügen gekennzeichnet. Diese Lösungsgefüge erhöhen zweifelsfrei die erreichbare Porosität aber offensichtlich nicht die Poreneintrittsradien, welche mit der Quecksilberporosimetrie ermittelt werden.

\subsubsection{Kapillare Wasseraufnahme}

Die kapillare Wasseraufnahme ist ein Maß für die Vernetzung von Kapillarporen in einem Gestein. Daneben besitzt die Wasseraufnahme für Marmore Bedeutung hinsichtlich der Verwitterung. Aufgrund der leichten Löslichkeit des Calcit-Kristalls, stellt eine hohe kapillare Aufnahme von Wasser ein besonderes Gefährdungspotenzial dar. Die untersuchten unverwitterten Marmorproben besitzen keine oder nur eine geringe kapillare Wasseraufnahme, welche sich unterhalb des Auflösvermögens der angewandten Meßmethode befindet. Dies ist ein weiterer Hinweis darauf, dass frische Marmore keine vernetzten Kapillarporen aufweisen. Für alle verwitterten Marmore hingegen ist eine deutliche kapillare Wasseraufnahme zu ermitteln, die in einer vollständigen Durchnässung der analysierten 
Würfelproben (65 mm Kantenlänge) resultiert. Die Menge des aufgenommenen Wassers variiert und spiegelt den Erhaltungszustand der Marmore wieder.

Exemplarisch ist das Wasseraufnahmeverhalten für die Proben des stark verwitterten Carrara und des mäßig verwitterten Lasa Marmors in Abb. 4.17 dargestellt. Hierbei ist die kapillare Wasseraufnahme als Anteil des Gesteins in Vol.-\% gegen die Zeit aufgetragen. Der Carrara Marmor besitzt mit 1,60 Vol.-\% eine ausgeprägtere Wasseraufnahme als der Lasa Marmor mit maximal 0,18 Vol.-\%. Diese Daten korrelieren mit den o.a. Porenraumdaten. Hierbei kann der Carrara Marmor schon aufgrund seiner höheren Gesamtporosität von 2,64 \% mehr Wasser aufnehmen als der Lasa Marmor mit 0,44 \%. Aber auch die Porenradienverteilung zeigt, dass der Carrara Marmor mit einem Porenradienmaximum von 2,371 $\mu \mathrm{m}$ effizienter Wasser absorbieren kann als der Lasa Marmor mit 0,237 $\mu \mathrm{m}$. Daraus resultiert für beide Proben auch ein abweichender Grad der Sättigung. Mit der durchgeführten Analytik kann durch kapillare Wasseraufnahme $62 \%$ des Carrara Marmors und nur maximal $40 \%$ des Porenraums des Lasa Marmors gesättigt werden (vgl. Tab. 4.2).
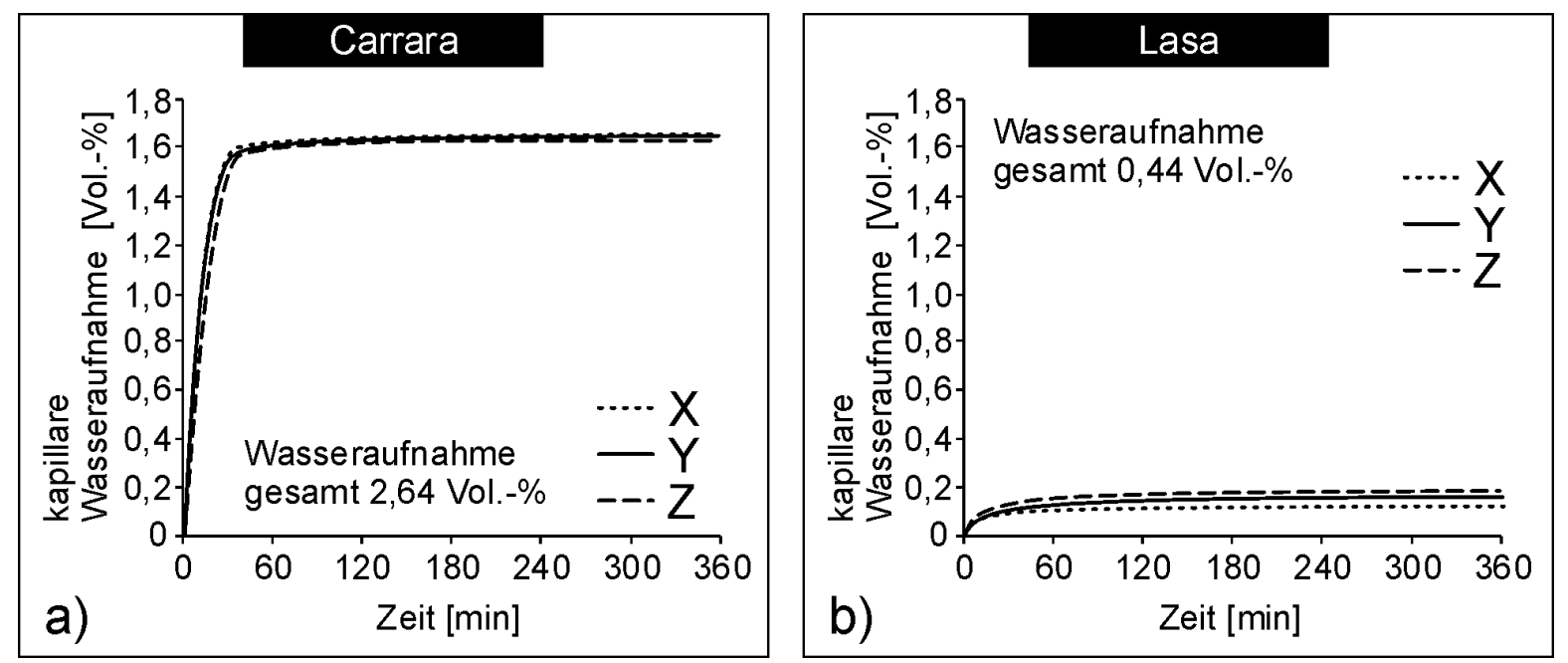

Abb. 4.17: An Würfeln in drei Richtungen ermitteltes kapillares Wasseraufnahmeverhalten a) der stark verwitterten Probe aus Carrara und b) der gering verwitterten aus Lasa Marmor.

Auffallend ist für alle verwitterten Marmore, dass die kapillare Wasseraufnahme relativ schnell erfolgt. Für die untersuchten Probengeometrien ist der Prozess bereits nach 0,5 bis 3 Stunden abgeschlossen. Für die verwitterten Proben sind zusätzlich die Wasseraufnahmekoeffizienten (w-Werte) in Tab. 4.2 aufgeführt. Die meisten Marmore weisen dabei Werte zwischen $0,06 \mathrm{~kg} / \mathrm{m} 2 * \sqrt{\mathrm{h}}$ und $0,19 \mathrm{~kg} / \mathrm{m} 2 * \sqrt{\mathrm{h}}$ auf. Ausnahme ist der stark verwitterte Carrara Marmor mit w-Werten zwischen $1,65 \mathrm{~kg} / \mathrm{m} 2 * \sqrt{\mathrm{h}}$ und $1,79 \mathrm{~kg} / \mathrm{m} 2 * \sqrt{\mathrm{h}}$.

\subsubsection{Ultraschallgeschwindigkeiten}

Marmore weisen im unverwitterten Gesteinszustand relativ hohe Ultraschallgeschwindigkeiten auf, die i.d.R. mit zunehmender Entfestigung stark abnehmen. Verantwortlich dafür ist die charakteristische gesteinsdurchdringende Rissbildung in Marmoren sowie die hohe Effizienz von Mikrorissen bei der Geschwindigkeitsreduktion (vgl. WEISS et al., 2002). Aufgrund des daraus resultierenden weiten Geschwindigkeitskontrastes sind verwitterungs- 
bedingte Geschwindigkeitsänderungen gut messbar. Somit erlauben Ultraschallanalysen Aussagen über den Grad der Entfestigung und ermöglichen eine diagnostische Beurteilung des Erhaltungszustands von Marmoren auch in der praktischen Anwendung vor Ort (vgl. KÖHLER, 1991). Der Vorteil des Verfahrens liegt in der zerstörungsfreien Durchführbarkeit und ist daher für die Qualitätssicherung von Naturwerksteinen und für den Einsatz im Bereich der Denkmalpflege geeignet. Die wesentliche Bedeutung kommt dabei der am Gebäude leicht zu messenden Kompressionswellengeschwindigkeit zu.

Tab. 4.2: Wasseraufnahmedaten der untersuchten verwitterten Marmortypen. Die angegebenen Werte wurden an jeweils einer Würfelprobe ermittelt.

\begin{tabular}{|c|c|c|c|c|c|c|}
\hline Marmortyp & Zustand & Richtung & $\begin{array}{c}\mathbf{W}_{\text {kap }} \\
\text { [Vol.-\%o] }\end{array}$ & $\begin{array}{l}\text { Porosität } \\
\text { [Vol.-\%] }\end{array}$ & Sättigung [\%] & $\begin{array}{c}\text { w-Wert } \\
{\left[\mathrm{kg} / \mathrm{m}^{2} * \sqrt{\mathrm{h}}\right]}\end{array}$ \\
\hline \multirow{6}{*}{ Carrara } & \multirow{3}{*}{ verwittert } & $\mathrm{X}$ & 0,13 & \multirow{3}{*}{0,53} & 24,3 & 0,08 \\
\hline & & Y & 0,13 & & 24,3 & 0,06 \\
\hline & & $\mathrm{Z}$ & 0,12 & & 23,6 & 0,07 \\
\hline & \multirow{3}{*}{$\begin{array}{c}\text { stark } \\
\text { verwittert }\end{array}$} & X & 1,66 & \multirow{3}{*}{2,64} & 62,7 & 1,79 \\
\hline & & Y & 1,64 & & 62,0 & 1,73 \\
\hline & & Z & 1,63 & & 61,8 & 1,65 \\
\hline \multirow{3}{*}{ Prieborn } & \multirow{3}{*}{ verwittert } & $X$ & 0,25 & \multirow{3}{*}{0,42} & 58,6 & 0,15 \\
\hline & & $\mathrm{Y}$ & 0,20 & & 47,4 & 0,09 \\
\hline & & Z & 0,23 & & 53,4 & 0,12 \\
\hline \multirow{3}{*}{ Kauffung } & \multirow{3}{*}{ verwittert } & $\mathrm{X}$ & 0,07 & \multirow{3}{*}{0,46} & 16,1 & 0,05 \\
\hline & & Y & 0,09 & & 18,5 & 0,05 \\
\hline & & Z & 0,13 & & 27,4 & 0,08 \\
\hline \multirow{3}{*}{ Grosskunzendorf } & \multirow{3}{*}{ verwittert } & $\mathrm{X}$ & 0,24 & \multirow{3}{*}{0,74} & 32,0 & 0,13 \\
\hline & & Y & 0,19 & & 25,2 & 0,11 \\
\hline & & Z & 0,12 & & 17,0 & 0,12 \\
\hline \multirow{3}{*}{ Lasa } & \multirow{3}{*}{ verwittert } & $\mathrm{X}$ & 0,11 & \multirow{3}{*}{0,44} & 26,2 & 0,14 \\
\hline & & $\mathrm{Y}$ & 0,15 & & 34,1 & 0,12 \\
\hline & & Z & 0,17 & & 39,7 & 0,17 \\
\hline \multirow{3}{*}{ Sterzing } & \multirow{3}{*}{ verwittert } & $X$ & 0,12 & \multirow{3}{*}{0,40} & 30,9 & 0,10 \\
\hline & & $\mathrm{Y}$ & 0,20 & & 50,0 & 0,19 \\
\hline & & Z & 0,10 & & 24,5 & 0,09 \\
\hline
\end{tabular}

Die absolute Geschwindigkeitsverteilung von elastischen Wellen in Gesteinen resultiert aus der mineralogischen Zusammensetzung, dem Gefüge sowie aus der Art und der Anordnung von Poren bzw. Rissen und deren Füllungen (z.B. Wasser oder Luft; SIEGESMUND, 1996). Um den Anteil der verwitterungsbedingten Rissbildung mittels Ultraschall charakterisieren zu können, ist es notwendig die Eigenschaften des rissfreien (intrinsisch) und des rissbehafteten Gesteins (rissbedingt) getrennt $\mathrm{zu}$ betrachten. Über die Ermittlung der Differenz beider Zustände kann die gesteinseigene Entfestigung sowie die bevorzugte Orientierung von Mikrorissen quantifiziert werden. Beim Vorhandensein von Porenflüssigkeiten, wie z.B. Wasser tritt eine Erhöhung der Ultraschallgeschwindigkeiten auf. 


\subsubsection{Intrinsische Ultraschallgeschwindigkeiten}

Die intrinsischen Ultraschallgeschwindigkeiten kennzeichnen diejenigen eines rissfreien Gesteins und werden durch die Kristalleigenschaften der Gesamtheit der gesteinsbildenden Minerale und ihrer Anordnung bedingt (vgl. SiEgeSMund, 1996). Dabei weisen Marmore intrinsisch eine mehr oder weniger ausgeprägte Anisotropie der elastischen Eigenschaften auf, d.h. die Ultraschallgeschwindigkeiten variieren mit der Richtung in der sie gemessen werden. Bei Calcit-Marmoren ist dies auf die Eigenschaften des Calcit-Einkristalls in Verbindung mit einer kristallographischen Vorzugsregelung der Kristalle (Textur) zurückzuführen. Die Kompressionswellengeschwindigkeiten des Calcit sind dabei extrem anisotrop und variieren zwischen $\mathrm{V}_{\mathrm{p}}=5,7 \mathrm{~km} / \mathrm{s}$ parallel und $\mathrm{V}_{\mathrm{p}}=7,7 \mathrm{~km} / \mathrm{s}$ senkrecht zur c-Achse (s. Abb. 2.2, DREYER, 1974). Beim Vorhandensein einer Textur pausen sich die Einkristalleigenschaften auf das Gesamtgestein durch und erzeugen so eine Richtungsabhängigkeit von $V_{p}$.

Die Ermittlung der intrinsischen Geschwindigkeiten und ihrer Anisotropie kann durch weitgehende Schließung der Risse mittels zunehmendem Umschließungsdruck erfolgen (BIRCH, 1960, 1961; DürRAst, 1997; RAsolofosaOn et al., 2000). Der Effekt der $\mathrm{V}_{\mathrm{p}^{-}}$ Erhöhung ist exemplarisch in Abb.4.18 für die verwitterte Probe aus Lasa Marmor dargestellt. Die Daten der drei orthogonalen Gefügerichtungen wurden aus einer vollständig dreidimensionalen Verteilung der P-Wellengeschwindigkeiten einer Kugelprobe entnommen, welche in Abhängigkeit vom Umschließungsdruck bis $200 \mathrm{MPa}$ bestimmt wurden. Detaillierte Beschreibungen dieses Verfahrens befinden sich in JAHNS (1995) und WEISS (1998).

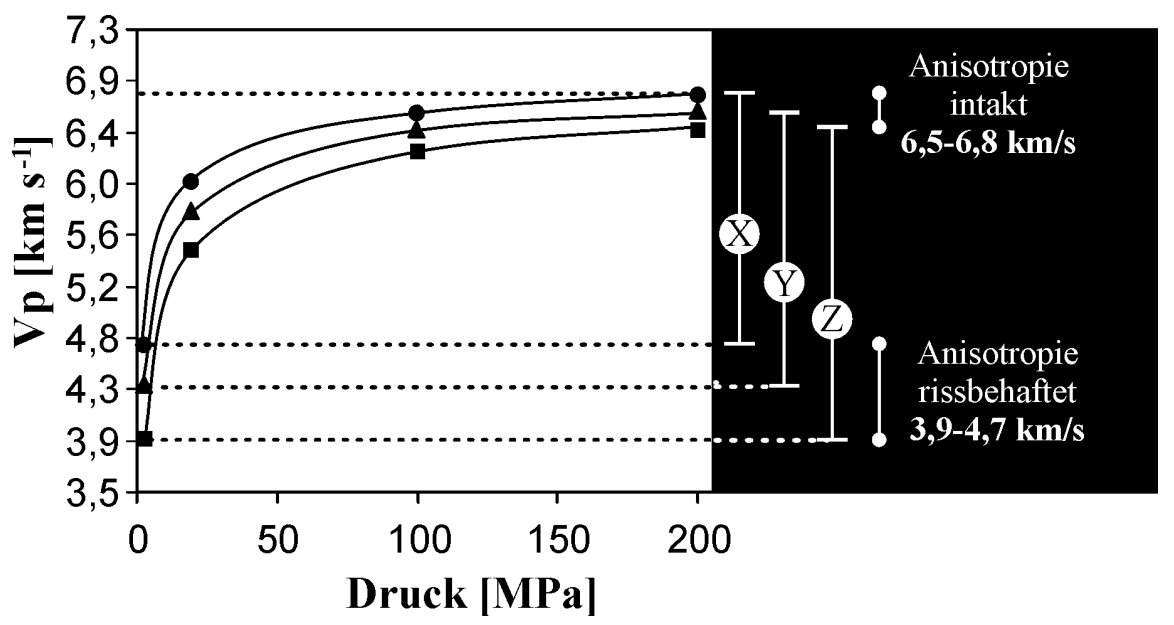

Abb. 4.18: Veränderung der Kompressionswellengeschwindigkeit eines Lasa Marmors bei zunehmenden Umschließungsdruck in drei orthogonalen Gefügerichtungen.

Mit zunehmendem Umschließungsdruck steigen die maximal Werte der Lasa Probe (Abb. 4.18) parallel zur X-Richtung von $4,7 \mathrm{~km} / \mathrm{s}$ (bei $2 \mathrm{MPa}$ ) auf $6,8 \mathrm{~km} / \mathrm{s}$ (bei $200 \mathrm{MPA}$ ). Besonders deutlich ist dabei der Geschwindigkeitsanstieg für die zweite Druckstufe bis $20 \mathrm{MPa}$. Die hohen Geschwindigkeiten, welche durch den Umschließungsdruck erzeugt werden, liegen im Bereich der Calcit-Einkristalldaten. Somit ist von einer weitgehenden Schließung der Mikrorisse auszugehen. 
Die aus den maximalen und minimalen $V_{\mathrm{p}}$-Werten für jede Druckstufe mit $A=\left(V_{\text {pmax }}\right.$ $\mathrm{V}_{\text {pmin }}$ ) / $\mathrm{V}_{\text {pmax }} * 100[\%]$ berechnete Anisotropie beträgt bei $2 \mathrm{MPa}$ ca. 17,0\% und reduziert sich jedoch bei zunehmendem Druck schnell. Für einen Umschließungsdruck von $200 \mathrm{MPa}$ verringert sich die $\mathrm{V}_{\mathrm{p}}$-Anisotropie auf 4,4\%. Damit ist die intrinsische Anisotropie für den Lasa Marmor relativ gering, was mit der ermittelten schwach bis mittleren Intensität der Textur dieses Marmors übereinstimmt (s. Kap. 4.2.1).

\subsubsection{Rissbedingte Ultraschallgeschwindigkeitsabnahme}

Verwitterte Marmore weisen im trockenen Zustand niedrigere Ultraschallgeschwindigkeiten auf als unverwitterte Marmore. Die Abnahme der Ultraschallgeschwindigkeiten resultiert dabei aus dem verwitterungsbedingten Mikrorisswachstum (z.B. WeIss et al., 2001). Die Differenz der $\mathrm{V}_{\mathrm{p}}$-Eigenschaften zwischen der rissfreien und der rissbehafteten Probe stellt dabei den rissbedingten Anteil der Ultraschallcharakteristik dar. In zahlreichen Arbeiten wird diskutiert inwieweit die Geschwindigkeitsdifferenz der rissbedingten Ultraschallgeschwindigkeitsabnahme als Maß für die Schädigung eines Marmors gewertet werden kann (z.B. KÖHLER, 1991 und WEISS et al., 2001). Generell können die im unverwitterten Zustand messbaren Kompressionswellengeschwindigkeiten von ca. $7 \mathrm{~km} / \mathrm{s}$ auf annähernd $1 \mathrm{~km} / \mathrm{s}$ für stark verwitterte Marmore reduziert sein (vgl. WEISS et al., 2000).

Die an Würfelproben im trockenen Zustand ermittelten $V_{\mathrm{p}}$-Werte sind für ausgewählte verwitterte Marmorproben in Tab. 4.3 aufgeführt. Das $V_{p}$-Spektrum variiert dabei zwischen $1,63 \mathrm{~km} / \mathrm{s}$ und $6,14 \mathrm{~km} / \mathrm{s}$. Bezüglich der Geschwindigkeitsanisotropien sind Variationen zwischen $9,5 \%$ und $49,3 \%$ festzustellen.

Tab. 4.3: $\mathrm{V}_{\mathrm{p}}$-Eigenschaften und Porositäten ausgewählter Marmorproben.

\begin{tabular}{|c|c|c|c|c|c|c|c|c|}
\hline \multirow[b]{2}{*}{ Marmortyp } & \multirow[b]{2}{*}{$\begin{array}{l}\text { Proben- } \\
\text { Zustand }\end{array}$} & \multirow[b]{2}{*}{$\begin{array}{l}\text { Porosität } \\
\text { [Vol.-\%] }\end{array}$} & \multicolumn{3}{|c|}{ TROCKEN } & \multicolumn{3}{|c|}{ WASSERGESÄTTIGT } \\
\hline & & & $\begin{array}{c}\mathrm{V}_{\text {pmax }} \\
{[\mathrm{km} / \mathrm{s}]}\end{array}$ & $\begin{array}{c}\mathrm{V}_{\mathrm{pmin}} \\
{[\mathrm{km} / \mathrm{s}]}\end{array}$ & $\begin{array}{l}A_{V p} \\
{[\%]}\end{array}$ & $\begin{array}{c}V_{\text {pmax }} \\
{[\mathrm{km} / \mathrm{s}]}\end{array}$ & $\begin{array}{c}V_{\text {pmin }} \\
{[\mathrm{km} / \mathrm{s}]}\end{array}$ & $\begin{array}{l}A_{V p} \\
{[\%]}\end{array}$ \\
\hline \multirow{3}{*}{ Carrara } & frisch & 0,20 & 6,42 & 6,16 & 4,1 & 6,76 & 6,62 & 2,1 \\
\hline & verwittert & 0,43 & 4,50 & 4,07 & 9,5 & 6,06 & 5,92 & 2,9 \\
\hline & $\begin{array}{c}\text { stark } \\
\text { verwittert }\end{array}$ & 2,12 & 2,44 & 1,63 & 33,0 & 4,83 & 4,67 & 3,3 \\
\hline Prieborn & verwittert & 0,55 & 3,97 & 2,93 & 26,1 & 6,16 & 5,74 & 6,8 \\
\hline \multirow{2}{*}{ Kauffung } & frisch & 0,23 & 6,58 & 5,43 & 17,8 & 7,17 & 6,31 & 12,0 \\
\hline & verwittert & 0,37 & 6,14 & 5,48 & 12,8 & 6,68 & 6,01 & 10,1 \\
\hline \multirow{2}{*}{ Grosskunzendorf } & frisch & 0,31 & 5,05 & 4,37 & 13,6 & 6,83 & 6,48 & 5,2 \\
\hline & verwittert & 0,77 & 4,84 & 2,45 & 49,3 & 6,50 & 5,81 & 10,5 \\
\hline \multirow{2}{*}{ Lasa } & frisch & 0,33 & 5,68 & 5,03 & 11,5 & 6,53 & 6,29 & 3,6 \\
\hline & verwittert & 0,45 & 4,48 & 3,77 & 16,0 & 5,92 & 5,64 & 4,7 \\
\hline \multirow{2}{*}{ Sterzing } & frisch & 0,30 & 6,40 & 4,87 & 23,8 & 6,87 & 6,06 & 11,7 \\
\hline & verwittert & 0,49 & 5,00 & 4,44 & 11,1 & 6,49 & 6,21 & 4,3 \\
\hline
\end{tabular}

Insbesondere hinsichtlich der praktischen Anwendung, ist es von ausschlaggebender Bedeutung, ob eine allgemein gültige Beziehung zwischen dem Verwitterungszustand und den Ultraschallgeschwindigkeiten von Marmoren existiert. KöHLER (1991) gründete auf umfangreichen $\mathrm{V}_{\mathrm{p}}$-Messungen an Carrara Marmoren eine Schadensklassifikation und definierte fünf Schadensklassen (Tab. 4.4). Ob diese Schadensklassifikation in sich konsistent 
ist und generell auch auf die untersuchten Marmorproben übertragen werden kann, soll im folgenden überprüft werden.

Die Herabsetzung der Ultraschallgeschwindigkeiten bei fortschreitender Verwitterung von Marmoren ist auf eine Zunahme der Rissdichte und ansteigende Rissweite zurückzuführen. Daneben besitzt die Art der Risse und die Verteilung im Gestein ein entscheidende Bedeutung. Nach Mikrobereichsuntersuchungen ist festzustellen, dass die jeweiligen Rissarten und -verteilungsmuster auf bestimmte Gefügeelemente zurückzuführen sind, wobei das Korngefüge hervorzuheben ist (vgl. Kap.4.2.2). Daher sollen im folgenden die Ergebnisse anhand der Korngefügetypen und der Mikrorissmuster diskutiert werden. $\mathrm{Zu}$ letzterem befindet sich in Abb. 4.19 eine schematische Darstellung.

Tab. 4.4: Schadensklassifikation auf der Basis von $\mathrm{V}_{\mathrm{p}}$-Daten für Marmor, aus Köhler (1991).

\begin{tabular}{|c|c|c|c|}
\hline Schadensklasse & $\mathrm{V}_{\mathrm{p}}[\mathrm{km} / \mathrm{s}]$ & Zustand & Porosität $\Phi[\%]$ \\
\hline 0 & $>5,0 \mathrm{~km} / \mathrm{s}$ & frisch & $<0,5$ \\
\hline I & $3,0 \mathrm{~km} / \mathrm{s}-5,0 \mathrm{~km} / \mathrm{s}$ & zunehmend porös & $1,3-0,5$ \\
\hline II & $2,0 \mathrm{~km} / \mathrm{s}-3,0 \mathrm{~km} / \mathrm{s}$ & absandend & $3,0-1,3$ \\
\hline III & $1,5 \mathrm{~km} / \mathrm{s}-2,0 \mathrm{~km} / \mathrm{s}$ & brüchig & $5,3-3,0$ \\
\hline IV & $<1,5 \mathrm{~km} / \mathrm{s}$ & zerfallen & $>5 \%$. \\
\hline
\end{tabular}

Für den Korngefügetyp I (Carrara, Prieborn und Lasa Marmor) ist eine relativ homogene, gesteinsdurchdringende Rissbildung an Korngrenzen $\mathrm{zu}$ beobachten (vgl. Abb. 4.19a). Das Rissverteilungsmuster des Korngefügetyp I entspricht vermutlich auch denjenigen der von Köhler untersuchten Carrara Marmore. Für die Marmore mit equigranular polygonalem Korngefüge ist daher auch eine gute Übereinstimmung zwischen den Schadensphänomenen und der Schadenklassifikation festzustellen. Danach ist die verwitterte Marmorprobe aus Prieborn, die aus makroskopischen Beobachtungen als zunehmend porös zu beschreiben ist, übereinstimmend mit den $V_{\mathrm{p}}$-Daten $\left(\mathrm{V}_{\mathrm{pmin}}=2,93 \mathrm{~km} / \mathrm{s} / \mathrm{V}_{\mathrm{pmin}}=3,97 \mathrm{~km} / \mathrm{s}\right)$ in die Schadensklasse I einzuordnen. Hingegen ist die stark verwitterte Probe aus Carrara Marmor als brüchig anzusehen und befindet sich, im Einklang mit den $\mathrm{V}_{\mathrm{p}}$-Werten $\left(\mathrm{V}_{\text {pmin }}=1,63 \mathrm{~km} / \mathrm{s} / \mathrm{V}_{\text {pmin }}=2,44 \mathrm{~km} / \mathrm{s}\right)$, in Schadensklasse III.

Bezüglich der ungleichkörnigen interlobaten Marmore aus Grosskunzendorf und Sterzing (Korngefügetyp II) ist neben der penetrativen Rissverteilung zusätzlich eine besonders weite Öffnung parallel verlaufender lokaler Risse, zumeist entlang intragranularer Vorzeichnungen, festzustellen (vgl. Abb. 19b). Dies macht sich in der $\mathrm{V}_{\mathrm{p}}$-Anisotropie des Grosskunzendorfer Marmors von bis zu $50 \%$ bemerkbar (s. Tab. 4.3). Die Entfestigung an diesen isolierten Rissen ist dabei so weit fortgeschritten, dass das Gestein an ihnen zerbricht und damit der Marmor als vollständig zerstört anzusehen ist. Bei Betrachtung der $\mathrm{V}_{\mathrm{p}}$-Werte parallel zu den Rissen sind jedoch noch Geschwindigkeiten von bis zu $4,8 \mathrm{~km} / \mathrm{s}$ festzustellen, womit das Gestein nur in Schadensklasse I einzuordnen wäre.

Der Kauffunger Marmor der den Korngefügetyp III repräsentiert, ist im Gegensatz zu den anderen Marmoren nicht durch ein gesteinsdurchdringendes Rissverteilungsmuster charakterisiert. Vielmehr handelt es sich nur um isolierte, das Gestein durchziehende Risse (vgl. Abb. 4.19c). Auch für diesen Marmor ist festzustellen, dass er aufgrund der starken 
Entfestigung an lokalen Rissen häufig auseinander bricht und damit als stark zerstört einzuordnen ist. Von den Ultraschallgeschwindigkeiten von über $6 \mathrm{~km} / \mathrm{s}$ ist er jedoch in die Schadensklasse 0 einzuordnen, womit es sich um einen frischen Marmor handelt.
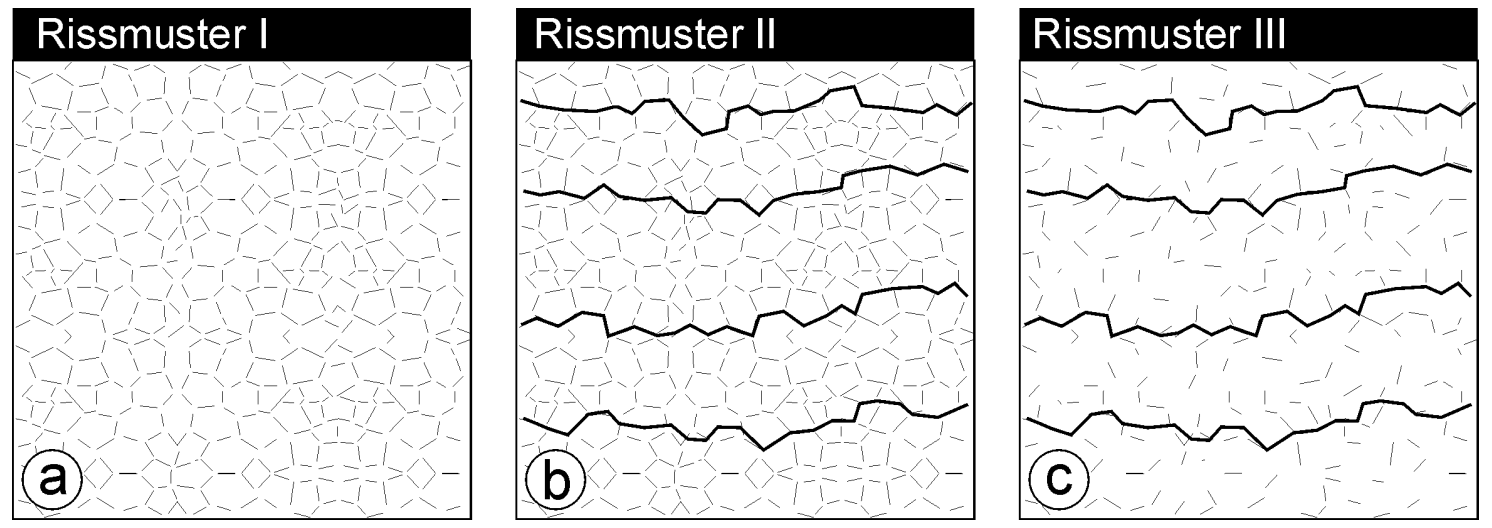

Abb. 4.19: Verschiedene Rissmuster der untersuchten Marmore: a) Gleichmäßig, das Gestein durchsetzende Mikrorisse typisch für equigranular polygonale Marmore des Korngefügetyp I. b) Zusätzlich zu den gesteinsdurchsetzenden Rissen sind häufig isolierte transgranulare Risse in den Marmoren des Korngefügetyp II zu ermitteln. c) Lokale transgranulare Risse dominieren den Korngefügetyp III.

Die Ergebnisse zeigen, dass die rissbedingte Herabsetzung von $V_{p}$ sehr unterschiedlich für Marmore sein kann, was nicht zwangsläufig durch den Verwitterungszustand bzw. durch die Dauer der Exposition bedingt ist. Eine Übereinstimmung mit der Schadensklassifikation nach Köhler liefern vor allem Marmore feiner bis mittlerer Körnung, die eine Entfestigung an Korngrenzen aufweisen (Korngefügetyp I). Ungleichkörnige interlobate Marmore oder Sonderfälle wie der Kauffunger Marmor können häufig isolierte Risse und Risssysteme aufweisen, an denen das Gestein zerbricht, die jedoch aufgrund ausgeprägter Anisotropien nur in bestimmten Richtungen detektierbar sind oder nur geringe Auswirkung auf die Ultraschallgeschwindigkeiten aufzeigen. Eine universale Überführung der Schadensklassifikation auf alle Marmore erscheint daher nicht sinnvoll. Insbesondere bei Marmoren, die lokale und gerichtete Risssysteme ausbilden, ist die Übertragung nur bedingt, d.h. nur mit Erfassung ihrer Anisotropien möglich.

\subsubsection{Einfluss der Wassersättigung}

Für wassergesättigte Marmorproben ist eine Zunahme der Ultraschallgeschwindigkeiten festzustellen. Die Ursache dieses Verhaltens beruht auf der begrenzten Kompressibilität von Wasser im Vergleich zu Luft (vgl. NuR \& Simmons, 1969). Das bedeutet, dass der Effekt des Porenraums beim Vorhandensein von Wasser reduziert ist und sich die PWellengeschwindigkeiten den intrinsischen annähern (vgl. WEISS et al., 2000).

Die an Würfelproben ermittelten P-Wellengeschwindigkeiten für den wassergesättigten Zustand ausgewählter Marmorproben können Tab. 4.3 entnommen werden. Die meisten der untersuchten verwitterten Marmore erreichen bei Wassersättigung sehr hohe Ultraschallgeschwindigkeiten zwischen 6 und $7 \mathrm{~km} / \mathrm{s}$. Ausnahmen sind die stark verwitterten Marmore aus Cararra und Grosskunzendorf, welche P-Wellengeschwindigkeiten unterhalb 
von $6 \mathrm{~km} / \mathrm{s}$ aufweisen. Diese Diskrepanz ist möglicherweise auf die geringere Geschwindigkeit von Kompressionswellen in Wasser und/oder auf eine nicht vollständige Sättigung des Porenraums zurückzuführen.

Es kann festgestellt werden, dass je stärker die Marmorproben verwittert und somit die Geschwindigkeiten im trockenen Zustand herabgesetzt sind, die Diskrepanz zwischen den aus den Einkristalldaten zu erwartenden und den experimentell ermittelten Daten zunimmt. Dies ist in Abb. 4.20 für Ultraschallgeschwindigkeiten, ermittelt an Würfelproben für den Carrara und Grosskunzendorfer Marmor, dargestellt. Die Daten zeigen eine deutliche Abhängigkeit der zu erreichenden Geschwindigkeiten im wassergesättigten Zustand von den Geschwindigkeiten unter trockenen Bedingungen. Dabei erreicht z.B. eine Carrara Probe, die im trockenen Zustand eine Geschwindigkeit von $V_{p}=2,0 \mathrm{~km} / \mathrm{s}$ aufweist, wassergesättigt nur $\mathrm{V}_{\mathrm{p}}=4,5 \mathrm{~km} / \mathrm{s}$.

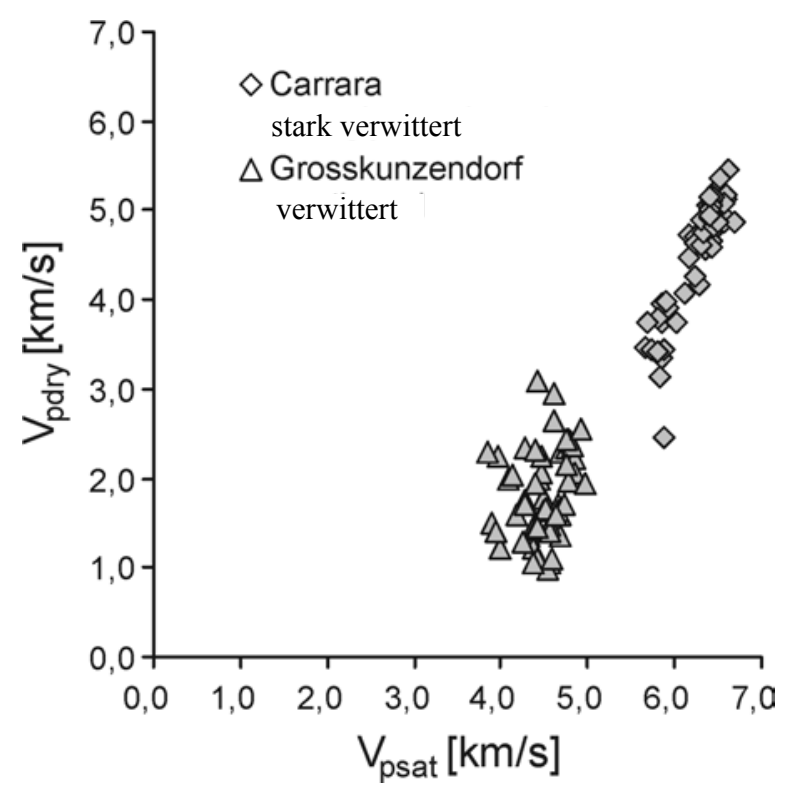

Abb. 4.20: Kompressionswellengeschwindigkeiten des trockenen gegen den wassergesättigten Zustand der stark verwitterten Marmore aus Carrara und Grosskunzendorf, ermittelt an Würfelproben.

Für die Marmore mittlerer Verwitterung, welche bei Wassersättigung sehr hohe Ultraschallwerte erreichen, ist festzustellen, dass auch die Geschwindigkeitsverteilungen mit denjenigen des rissfreien Gesteins weitgehend übereinstimmen. Dies ist im Folgenden am Beispiel der P-Wellengeschwindigkeitseigenschaften $\left(\mathrm{V}_{\text {psat }}\right)$ für wassergesättigte Proben des Lasa und des Prieborner Marmors dargestellt (Abb. 4.21a,e). Hierbei handelt es sich um Marmore der unterschiedlichen Texturmuster des c-Achsen- und des a-Achsenfasertyps. Die Daten wurden aus einer vollständigen dreidimensionalen Verteilung der PWellengeschwindigkeiten (aus 154 unabhängigen Richtungen) bestimmt. Zum Vergleich sind in Abb. 4.21c,d,g,h die jeweiligen Texturen für die c- und a-Achsenverteilung der entsprechenden Marmorproben aufgeführt.

Beide Proben zeigen eine Übereinstimmung der Ultraschallgeschwindigkeitsverteilung mit der jeweiligen Textur. Dabei fallen die Minima der P-Wellengeschwindigkeiten auf die 
Bereiche höchster Intensität der c-Achsenverteilung. Ebenso gleichen die Bereiche hoher Geschwindigkeiten dem Verteilungsmuster der a-Achsen. Die in der Textur ausgebildeten Gürtelverteilungen der a-Achsen des Prieborner ebenso wie die Gürtelverteilung der c-Achsen des Lasa Marmors kommen in den Geschwindigkeitsmustern jedoch nur undeutlich zum Vorschein. Für beide Proben wird dies durch eine Überlagerung der Gürtelverteilungen durch Submaxima hervorgerufen.

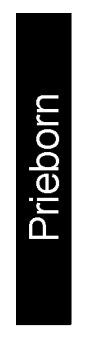

a)
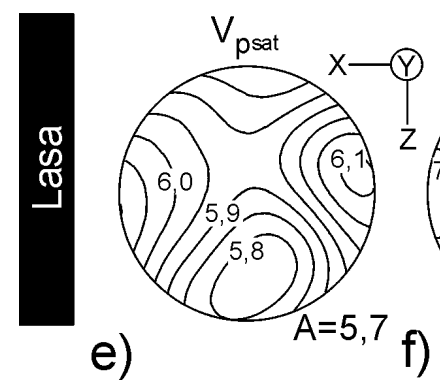

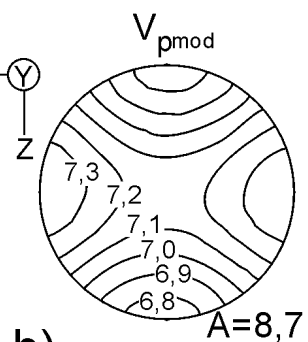

b)

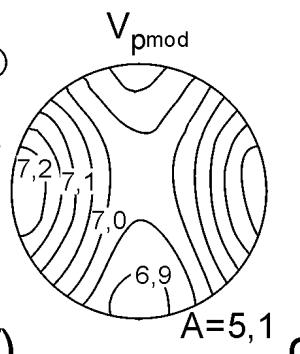

g)

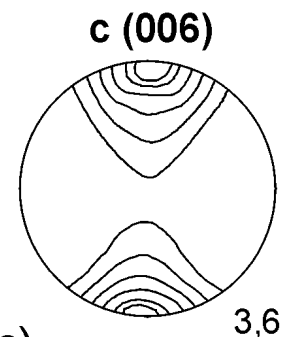

C)

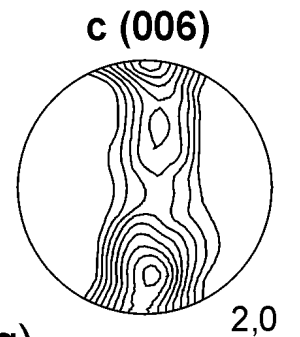

2,0

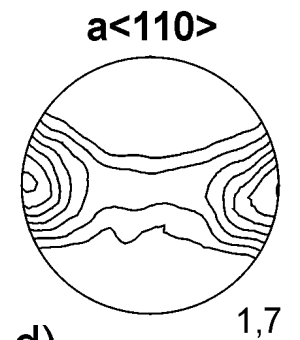

d)

h)

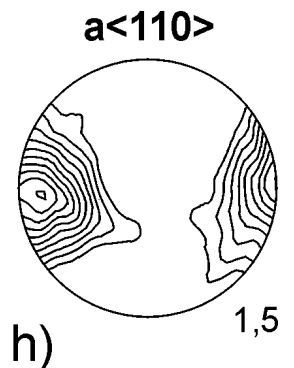

1,5

Abb. 4.21: Intrinsische Geschwindigkeitsverteilungen (Tensordarstellung) für die verwitterten Proben aus Lasa und Prieborner Marmor. a,e) an wassergesättigten Kugelproben experimentell ermittelt $\left(\mathrm{V}_{\mathrm{psat}}\right)$ und $\left.\mathrm{b}, \mathrm{f}\right)$ aus der Textur nach Voigt berechnete Geschwindigkeitsverteilungen sowie c,d,g,h) c-Achsen-, unten a-Achsenverteilung der jeweiligen Marmorprobe. Die Isolinien repräsentieren $\mathrm{km} / \mathrm{s}(\mathrm{a}, \mathrm{b}, \mathrm{e}, \mathrm{f})$ und ein Vielfaches der Gleichverteilung [VGV] (c,d,g,h). Schmidt'sches Netz, untere Halbkugel.

Zur Überprüfung wurde zusätzlich aus den Daten der Textur die Ultraschallgeschwindigkeitsverteilung nach VoIGT (1928) berechnet ( $\left.\mathrm{V}_{\text {pmod }}\right)$. Die Ergebnisse in Abb. 4.21b,f zeigen ein vergleichbares Muster der $V_{p}$-Verteilung zu den experimentellen Daten. Weiterhin ist auch eine annähernde Übereinstimmung der Anisotropie sowohl für den Prieborner Marmor mit $A_{V p s a t}=7,9 \%$ und $A_{V p m o d}=8,7 \%$ als auch für die Probe des Lasa Marmors mit $A_{V p s a t}=5,7 \%$ und $A_{V p m o d}=5,1 \%$ festzustellen. Die errechneten $V_{p}$-Werte liegen jedoch mit ca. $1 \mathrm{~km} / \mathrm{s}$ deutlich über den gemessenen.

\subsubsection{Quantifizierung der Vorzugsorientierung von Mikrorisssystemen}

Die Vorzugsorientierung von Mikrorissen gibt an, in welche Richtungen der Marmor durch die Verwitterung bevorzugt geschädigt wurde. Von besonderem Interesse ist hierbei welche Gefügeelemente für eine orientierte Rissbildung verantwortlich sind. Weiterhin besitzt die Kenntnis des Einflusses der bevorzugt orientierten Mikrorisse auf die Richtungsabhängigkeit der Ultraschallgeschwindigkeiten auch praktische Bedeutung. Dies ist zum Beispiel für bestimmte Untersuchungen wie die Ultraschalltomographie zur Charakterisierung des 
Erhaltungszustandes von Marmoren der Fall (s. Kap. 8.2; vgl. SIEGESMUND et al., 2000a; RUEDRICH et al., 2001b).

Die quantitative Ermittlung der bevorzugten Orientierung von Mikrorissen erfolgte in der vorliegenden Arbeit aus der Differenz der Ultraschallgeschwindigkeitsverteilung des rissfreien (unverwitterten) und des rissbehafteten (verwitterten) Zustands der Marmore (s. WEISS et al., 2000). Wie oben angeführt, kann der rissfreie intrinsische Zustand eines Marmors nur annähernd gemessen werden. In der vorliegenden Arbeit erfolgte eine näherungsweise Erfassung der bevorzugten Orientierung von Mikrorissen für die Marmore schwacher bis mittlerer Verwitterung aus der $\mathrm{V}_{\mathrm{p}}$-Differenz des wassergesättigten und des trockenen Zustands von Kugelproben. Zur Darstellung der Rissverteilungen eignen sich dabei sog. $\Delta \mathrm{V}_{\mathrm{p}}$-Diagramme (z.B. SIEGESMUND et al., 1993).

In Abb. 4.22 sind exemplarisch die ermittelten Geschwindigkeitsverteilungen für den wassergesättigten und den trockenen Zustand sowie die errechnete Differenzpolfigur für den Sterzinger, den Carrara und den Kauffunger Marmor dargestellt. Für die verschiedenen Proben sind dabei unterschiedliche Rissverteilungsmuster $\mathrm{zu}$ ermitteln, die durch eine Verstärkung und/oder Veränderung der intrinsischen Anisotropie charakterisiert sind.

Der Sterzinger Marmor stellt ein Beispiel für eine reine Verstärkung der intrinsischen Anisotropie durch die verwitterungsbedingte Rissbildung dar. Dies ist am vergleichbaren Verteilungsmuster von $\mathrm{V}_{\text {psat }}$ und $\Delta \mathrm{V}_{\mathrm{p}}$ erkennbar (Abb. 4.22a-c). Im Vergleich der beiden Polfiguren ist eine Übereinstimmung der Bereiche höchster Geschwindigkeiten von $\mathrm{V}_{\mathrm{psat}}=6,2 \mathrm{~km} / \mathrm{s}$ mit denen einer geringen Abnahme von $\Delta \mathrm{V}_{\mathrm{p}}=1,3 \mathrm{~km} / \mathrm{s}$ festzustellen. Bereiche mit ursprünglich relativ niedrigen Kompressionswellengeschwindigkeiten von $\mathrm{V}_{\text {psat }}=5,7 \mathrm{~km} / \mathrm{s}$ weisen eine starke Reduzierung von $\Delta \mathrm{V}_{\mathrm{p}}=1,8 \mathrm{~km} / \mathrm{s}$ bedingt durch die Risssysteme auf. Die intrinsische Anisotropie wird dabei durch die rissgenerierte Anisotropie um $13 \%$ verstärkt. Nach mikrogefügekundlichen Untersuchungen weist der Sterzinger Marmor häufig offene Risse entlang von Zwillingslamellen auf. Dabei folgen die Zwillinge im Calcit-Kristall zumeist den e-Flächen, welche bevorzugt senkrecht zum cAchsenmaximum orientiert sind (vgl. Abb. 4.12d). Für den Sterzinger Marmor ist somit anzunehmen, dass die Öffnung von Zwillingsgrenzen für die beobachtete Anisotropieverstärkung verantwortlich ist.

Die $\Delta \mathrm{V}_{\mathrm{p}}$-Polfigur der ausgewählten Carrara Marmorprobe (Abb. 4.22d-f) weist ein modifiziertes Rissverteilungsmuster aus, d.h. es liegt keine reine Verstärkung der Anisotropie des rissfreien Gesteins vor. Das Maximum des $\Delta \mathrm{V}_{\mathrm{p}}$-Diagramms zeigt eine Geschwindigkeitsdifferenz von $\Delta \mathrm{V}_{\mathrm{p}}=1,6 \mathrm{~km} / \mathrm{s}$. Die niedrigsten Geschwindigkeitsänderungen treten in einem Gürtel in der XY-Richtung auf. Ein Subminimum ist parallel zur X-Richtung mit $\Delta \mathrm{V}_{\mathrm{p}}=1,2 \mathrm{~km} / \mathrm{s}$ festzustellen. Damit kann auch für die Probe aus Carrara Marmor beobachtet werden, dass die größte Herabsetzung von $V_{p}$ parallel zur bevorzugten Orientierung der kristallographischen c-Achsen auftritt. Die Modifizierung des $\mathrm{V}_{\mathrm{p}}$-Musters ist hier möglicherweise auf den Einfluss der bevorzugten Kornformorientierung zurückzuführen (vgl. Kap. 4.2.1). Es erscheint naheliegend, dass insbesondere für Marmore des Korngefügetyp I, welcher eine reine Korngrenzenentfestigung aufweist, die Kornformregelungen zu einer bevorzugten Orientierung von Rissen beiträgt. 


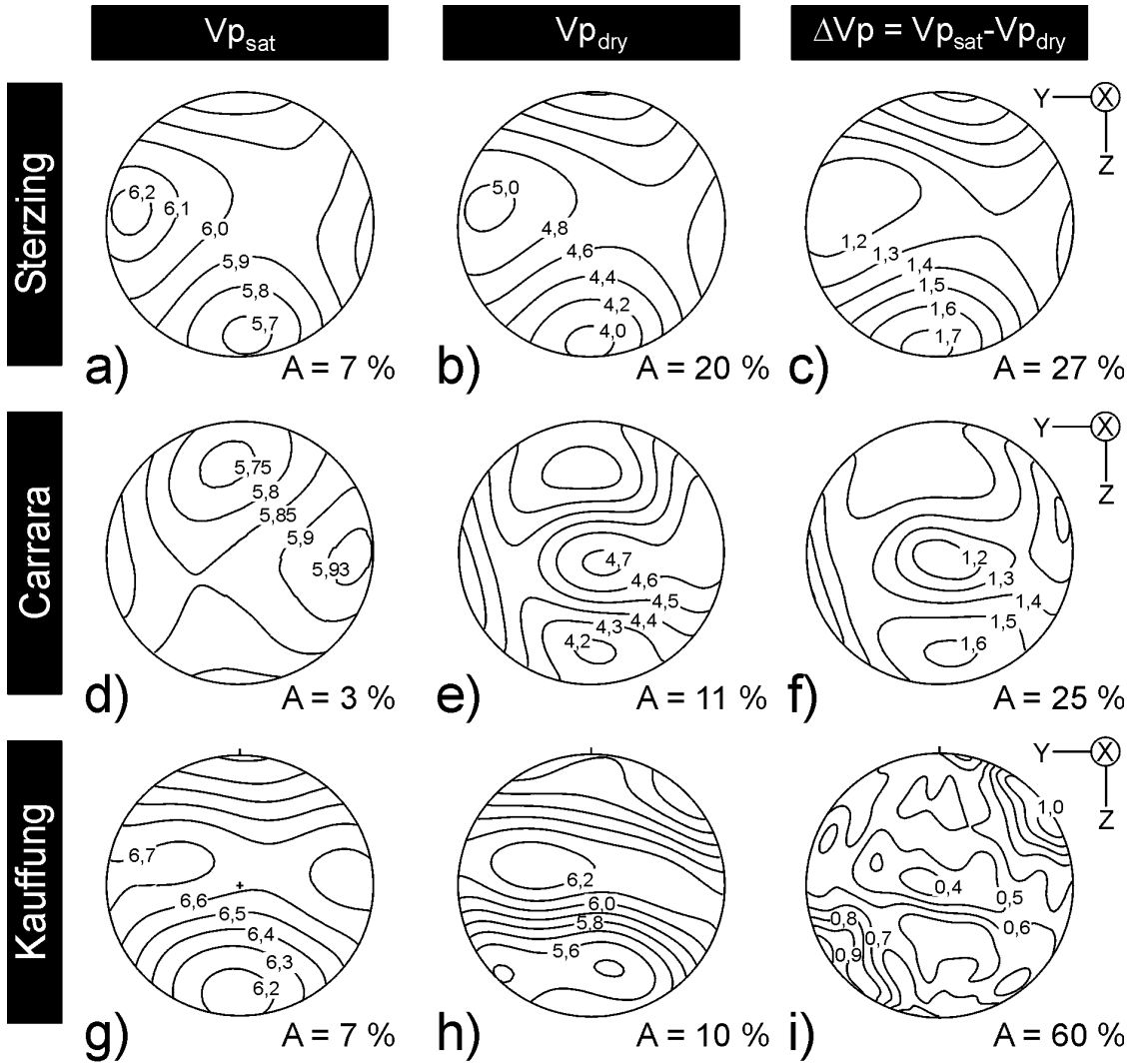

Abb. 4.22: Geschwindigkeitsverteilungen von Kugelproben im trockenen $\left(\mathrm{V}_{\text {pdry }}\right)$ und wassergesättigten $\left(\mathrm{V}_{\mathrm{psat}}\right)$ Zustand sowie die Geschwindigkeitsdifferenzen $\left(\Delta \mathrm{V}_{\mathrm{p}}\right.$-Diagramme $)$ der verwitterten Marmore aus Sterzing, Carrara und Kauffung (Schmidt'sches Netz, untere Halbkugel).

Für den Kauffunger Marmor ist ein weitgehend abweichendes Verteilungsmuster der Risse in Bezug auf die intrinsische Anisotropie festzustellen (Abb. 4.22g-i). Die $\Delta \mathrm{V}_{\mathrm{p}}$-Polfigur zeigt ein heterogenes Bild, mit der größten Herabsetzung von ca. $\Delta \mathrm{V}_{\mathrm{p}}=1,0 \mathrm{~km} / \mathrm{s}$ zwischen der $\mathrm{Y}$ und Z-Richtung in der YZ-Ebene. Die niedrigeren $\Delta \mathrm{V}_{\mathrm{p}}$-Werte befinden sich um $90^{\circ}$ versetzt in einem breiten Gürtel. Dabei variieren die Werte mit lokalen Submaxima zwischen $\Delta \mathrm{V}_{\mathrm{p}}=0,4 \mathrm{~km} / \mathrm{s}$ und $\Delta \mathrm{V}_{\mathrm{p}}=0,8 \mathrm{~km} / \mathrm{s}$. Im Gegensatz zu den beiden ersten Verteilungsmustern ist für den Kauffunger Marmor festzustellen, dass die größte $V_{p}$-Reduktion diagonal zur Regelung der c-Achsen auftritt. Nach mikrogefügekundlichen Untersuchungen kann für den Kauffunger Marmor die Hauptentfestigung entlang präexistierender, verheilter und mineralisierter bzw. versiegelter Risse festgestellt werden. Hierbei ist ein signifikanter Anteil senkrecht aufeinander stehender Risse, diagonal zur Foliation festzustellen (vgl. Tafel 3, Abb. 6). Es ist davon auszugehen, dass diese Risssysteme, welche lokal sehr unterschiedlich orientiert sein können, den wesentlichen Einfluss auf die Verteilung der $V_{p}$-Reduktion besitzen.

\subsubsection{Zusammenhang zwischen $\mathrm{V}_{\mathrm{p}}$ und der Porosität}

Für die Diagnostik des Erhaltungszustandes von Marmoren mittels Ultraschall stellt sich die Frage, welchen Einfluss die Porosität auf die Geschwindigkeiten ausübt. Zur Korrelation der Ultraschallgeschwindigkeiten mit dem Porenraum kristalliner Gesteine gibt es in der Literatur zahlreiche Modellvorstellungen, die aus verschiedenen Wissenschaftsdisziplinen stammen. Im 
Folgenden sollen zwei unterschiedliche Ansätze mit den in dieser Arbeit ermittelten Daten überprüft werden. Hierbei handelt es sich zum einen um die Arbeitshypothese von KöHLER (1991) und zum anderen um den Modellansatz von WeISS et al. (2000), welcher auf den mathematischen Grundlagen von O`CONNEL \& BUDIANSKY (1974) beruht.

Die Beziehung von $V_{p}$ zur Porosität nach KöHLER (1991) beruht auf Messungen von Carrara Marmor in unterschiedlichsten Erhaltungszuständen. Auf der Basis dieser empirischen Daten entwickelte er eine Korrelationsfunktion zwischen $V_{p}$ und der Porosität (Formel 4.1).

Formel 4.1:

$$
V_{p} \sim \sqrt{\frac{12}{\Phi}}
$$

Nach dem Modellansatz von WeISS et al. (2000) ist die Geschwindigkeitsreduktion als eine Funktion der Rissgeometrie zu verstehen. Das grundlegende Prinzip beruht darauf, dass eine vorhandene Porosität durch bestimmte Typen von ellipsoiden Rissen gebildet wird. Dabei wird die Rissgeometrie definiert durch das Längenverhältnis von Kurzachse zu Langachse (aspect ratio). Kugelporen würden dabei einen aspect ratio von 1 und flache Poren von kleiner 1 besitzen (s. Abb. 4.23c). Die bei kristallinen Marmoren durch die Verwitterung hervorgerufene starke Geschwindigkeitsherabsetzung kann dabei nur durch sehr flache Risse hervorgerufen werden (vgl. WeISS et al., 2001, 2002; s. Abb. 4.23c).

Die an 123 Würfeln für ausgewählte Marmorproben ermittelten $\mathrm{V}_{\mathrm{p}}$-Daten sind in Abb. 4.23a,b als Funktion der Porosität des jeweiligen Probenkörpers im trockenen und wassergesättigten Zustand aufgetragen. Zusätzlich sind in die Diagramme beide Modellansätze eingefügt (vgl. WEISS et al., 2001). Im trockenen Zustand weisen die Proben Geschwindigkeiten zwischen $7,0 \mathrm{~km} / \mathrm{s}$ und $2,0 \mathrm{~km} / \mathrm{s}$ auf (s. Abb. 4.23a). Für die meisten Proben sind dabei niedrige Porositäten festzustellen. Sie befinden sich mehr oder weniger auf dem nach dem Modellansatz von O'CONNEL \& BUDIANSKY (1974) vorhergesagten Bereich von starker Ultraschallabnahme und geringer Porositätserhöhung die nur durch sehr flache Risse mit einem aspect ratio um 0,005 hervorgerufen werden können. Ausnahmen stellen die stark verwitterte Probe des Carrara und die des Grosskunzendorfer Marmors dar, die sich bei einem aspect ratio von 0,01 befinden.

Beide Marmore zeigen nach Mikrobereichsanalysen eine Modifikation der Rissgeometrien in Form von Lösungserscheinungen (vgl. Kap. 4.2.2). Diese erhöhen die Porosität besitzen jedoch scheinbar keinen ausgeprägten Einfluss auf die Ultraschallgeschwindigkeiten. Für den trockenen Zustand ist festzustellen, dass das Verhältnis von Ultraschall zu Porosität durch den Trend der stark verwitterten Carrara Probe auch der von Köhler entwickelten empirischen Korrelationsfunktion folgt. Es kann davon ausgegangen werden, dass Marmore die eine rein mechanische Mikrorissbildung aufweisen, den Modellansätzen von O`CONNEL \& BUDIANSKY (1974) folgen und dabei eine sehr geringe Porositätszunahme eine starke Geschwindigkeitsabnahme bedingt. Ab einer bestimmten Entfestigung können jedoch Lösungsprozesse wirksam werden, welche die Mikrorisse modifizieren und einen gegenläufigen Trend erzeugen. Dabei korreliert eine starke Porositätszunahme mit einer relativ geringen Geschwindigkeitsreduktion. 

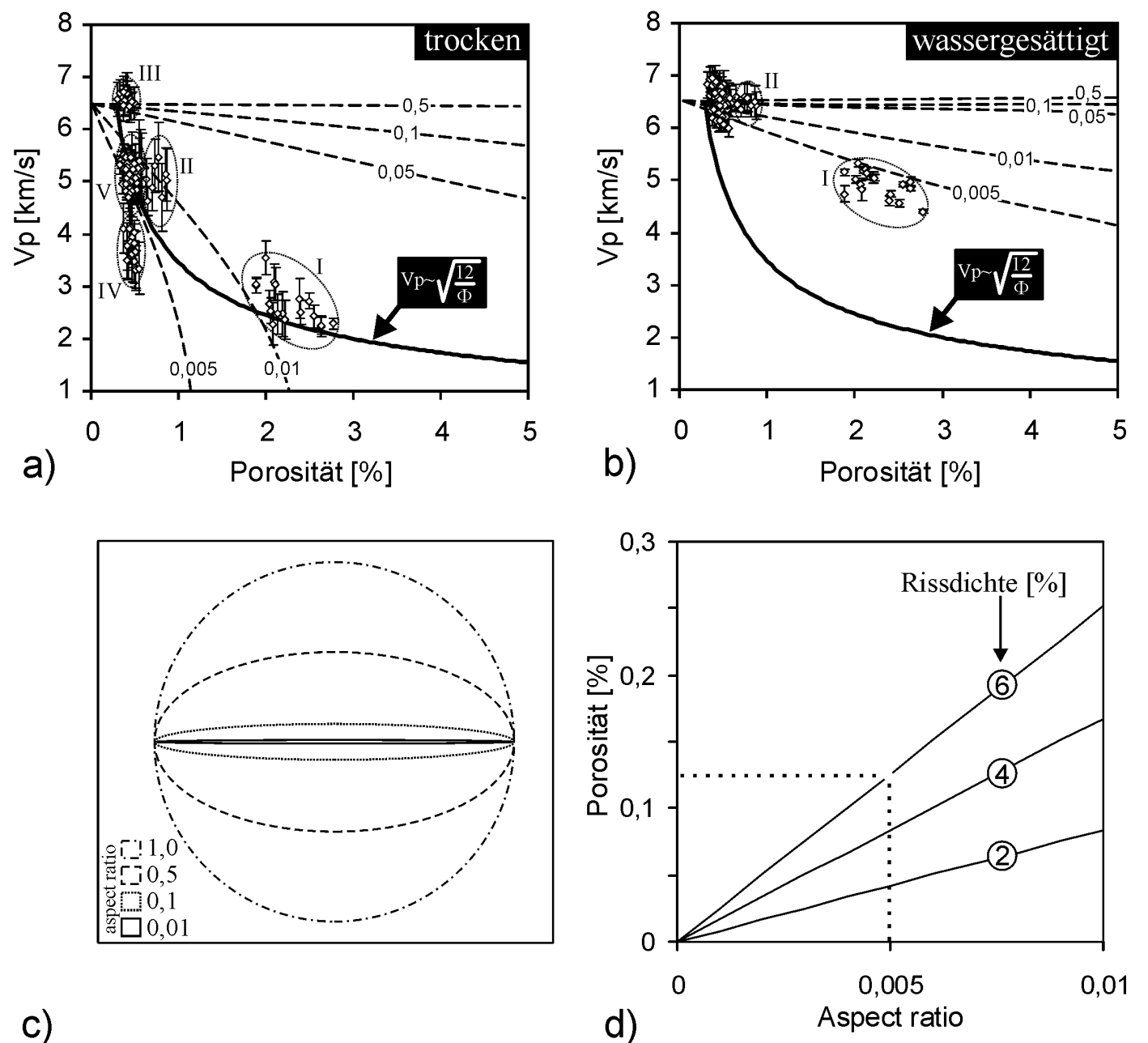

c)

Abb. 4.23: $\mathrm{V}_{\mathrm{p}}$-Eigenschaften und Porositäten ausgewählter Marmorproben: a,b) Mittlere Geschwindigkeit von Kompressionswellen verschiedener Marmorproben in Abhängigkeit von der Porosität und des Probenzustandes ( $\mathrm{I}=$ Carrara und II $=$ Grosskunzendorf beide stark verwittert, $\quad$ III $=$ Kauffung, $\quad$ IV = Prieborn, $\quad \mathrm{V}=$ Lasa, Sterzing, Carrara mittlerer Verwitterungszustand). Die Anisotropie der jeweiligen Probe ist durch Fehlerbalken angegeben. Die Geschwindigkeits-/Porositätskurve von KÖHLER (1991, durchgezogene Linie) und die theoretische Voraussage nach dem Modell von O'Connel \& Budiansky (1974, gestrichelte Linien) sind zum Vergleich eingetragen; c) schematische Darstellung des aspect ratios, d.h. der Porengeometrie, die in die Modellierungen nach O'Connel \& Budiansky eingegangen sind. Bereits bei einem aspect ratio von 0.01 sind die Poren extrem flach. d) Theoretische Berechnung des Porositätszuwachses als Folge thermischer Rissbildung für verschiedene Rissdichten. Eine Rissdichte von 6\% (s. punktierte Linie), ist durchaus realistisch für thermisch geschädigte Marmore, ergibt lediglich einen Anstieg der Porosität von $0,125 \%$ (Abbildung verändert nach WEISS et al., 2001).

Für die Proben im wassergesättigten Zustand ist der Trend der experimentellen Daten deutlich flacher (s. Abb. 4.23b). Dabei liegen die Daten des Carrara Marmors abweichend vom trockenen Zustand bei einem aspect ratio von 0,005. Die fehlende Übereinstimmung ist darauf zurückzuführen, dass stark verwitterte Marmore selbst bei Wassersättigung niedrigere Geschwindigkeiten aufweisen als diejenigen im rissfreien Zustand. Somit stimmen diese nicht mehr mit den Modellvorstellungen von O`CONNEL \& BUDIANSKY (1974) überein (vgl. WeISS et al., 2002). 


\subsubsection{Zugfestigkeitsanalyse mittels Brazil-Test}

Die Zugfestigkeit eines Naturwerksteins ist ein Maß für die Kohäsion seines Korngefüges. Dabei weisen kristalline Marmore selbst im unverwitterten Zustand, im Vergleich zu anderen kristallinen Gesteinen, relativ geringe Zugfestigkeiten auf (vgl. FLEISCHER, 2002 und StROHMEYER \& SiEgESMUND, 2002). Neben dem allgemeinen Einfluss der mineralogischen Zusammensetzung ist die Festigkeit bei Zugbelastung aber auch vom Gefügeinventar abhängig (BROSCH et al., 2000). Dies ist darauf zurückzuführen, dass ein erzwungener Zugbruch bevorzugt entlang von Schwächezonen verläuft. Dabei schwächen insbesondere vorhandene offene Mikrorisse den Gesteinskörper, da an ihnen Spannungsspitzen auftreten und sich der Bruch ausbreitet (PECK et al., 1985). Für kristalline Marmore sind weitere Gefügeelemente wie die Korngröße, die Kornverzahnung und die Textur wesentliche Einflussparameter, die das mechanische Verhalten bei Zugbeanspruchung beeinflussen. Da die Gefügeparameter häufig Anisotropien ausbilden, besitzen Marmore zumeist eine deutliche Richtungsabhängigkeit der Zugfestigkeiten.

Um die gefügeabhängige Spaltzugfestigkeit und ihre Anisotropie zu ermitteln, wurden in der vorliegenden Arbeit exemplarisch zwei gering verwitterte Marmorproben der Korngefügetypen I (Carrara) und II (Sterzinger) untersucht (vgl. Kap. 4.2.1). Die Analysen wurden an liegenden Probezylindern (Zylinderachse parallel der X-Richtung) durchgeführt. Die Auswahl der Probenrichtung wurde auf die Lage der Kornformregelung und der Textur ausgerichtet. Die Ermittlung der Spaltzugfestigkeitsanisotropie erfolgte durch Messungen in 13 unabhängigen Richtungen, wobei die Belastungsebene um die Zylinderachse in $15^{\circ}$ Schritten gedreht wurde (vgl. Abb. 4.24). Die $90^{\circ}$-Richtung zeichnet hierbei das Streichen der Foliation nach.
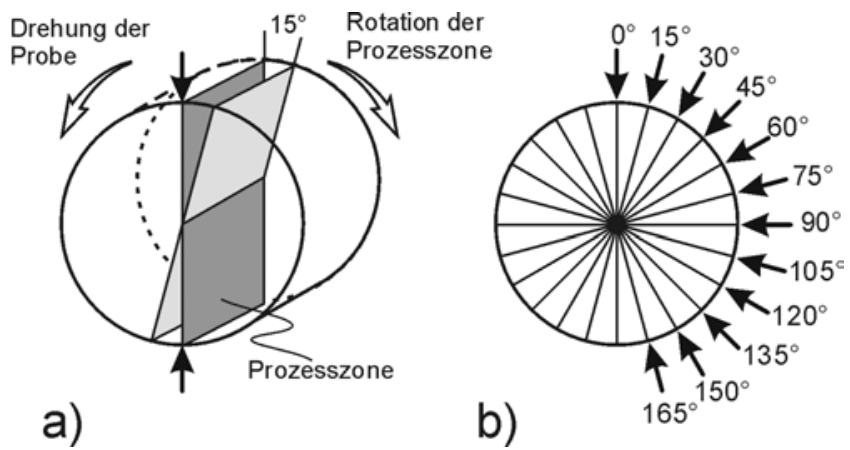

Abb. 4.24: Ermittlung der Richtungsabhängigkeit der Spaltzugfestigkeiten: a) Rotationsprinzip der Probenkörper und damit verbundene Wanderung der Prozesszone sowie b) gemessene Winkel welche das Streichen der Prozesszone angeben.

Die mit dem Brazil-Test ermittelten Spaltzugfestigkeiten sind in Abb. 4.25a,e dargestellt. Die aufgetragene Richtung gibt dabei das Streichen der Belastungsebene also die Lage der Prozesszone an, wobei die Zugspannungsrichtung senkrecht zu dieser liegt. Das Minimum der Spaltzugfestigkeit des untersuchten Carrara Marmors befindet sich bei $3.4 \mathrm{MPa}$, wohingegen das Maximum bei $5.6 \mathrm{MPa}$ liegt (s. Abb. 4.25a). Die mittels $A=\left(\sigma_{Z \max }-\right.$ $\left.\sigma_{Z \min }\right) / \sigma_{\mathrm{Zmax}} * 100[\%]$ ermittelte Anisotropie erreicht dabei einen Wert von $\mathrm{A}=39 \%$. Für den verwitterten Sterzinger Marmor wurden höhere Werte zwischen 4.8 $\mathrm{MPa}$ und $7.9 \mathrm{MPa}$ 
ermittelt. Die Anisotropie ist mit $\mathrm{A}=38 \%$ vergleichbar mit der des Carrara Marmors (s. Abb. 4.25e).
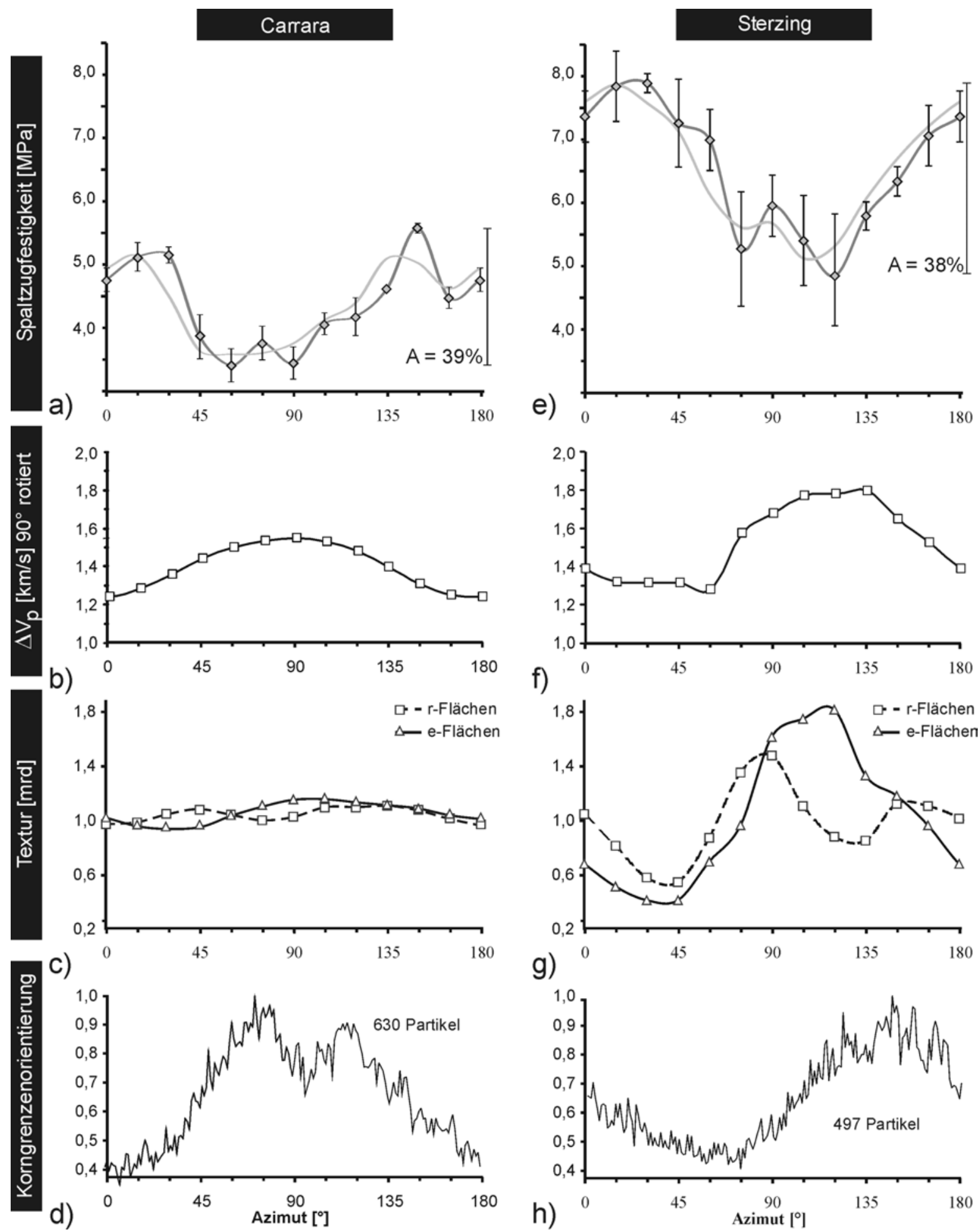

Abb. 4.25: Richtungsabhängigkeit der Spaltzugfestigkeiten und Korrelation mit Gefüge- und Ultraschalldaten für den Carrara und Sterzinger Marmor: a,e) Spaltzugfestigkeiten, $b, f) \Delta \mathrm{V}_{\mathrm{p}^{-}}$ Werte ermittelt aus Kugelmessungen, c,g) Textur der $\mathrm{r}\{104\}$ und e $\{018\}$ Kristallflächen sowie d,h) bevorzugte Korngrenzen Orientierung ( 1 = häufigste Belegung). Hinweis: Für die einzelnen Parameter ist das Streichen der Belastungszone (a,e) bzw. der Flächen (c,d,g,h) angegeben und für b,f die Richtung der Durchschallung um $90^{\circ}$ gedreht. 
Um zu ermitteln, welche Schwächezonen der Marmore durch den Spaltzugversuch aktiviert wurden, erfolgte an Dünnschliffen ausgewählter Bruchflächen die Untersuchung des Bruchbildes. Die Ergebnisse sind in Abb. 4.26 dargestellt und zeigen, dass der erzwungene Zugbruch eine deutliche Abhängigkeit vom Korngefüge des jeweiligen Gefügetyps aufweist. Für den feinkörnigen Carrara Marmor mit polygonalen Kornformen und vorwiegend geraden Korngrenzen ist festzustellen, dass der erzeugte Zugriss bevorzugt an Korngrenzen und nur selten intrakristallin verläuft (Abb. 4.26a). Im zweidimensionalen Anschnitt ist der Bruch auf ca. $83 \%$ der Strecke entlang von Korngrenzen entwickelt, wo hingegen nur auf ungefähr $17 \%$ intrakristalline Flächen aktiviert wurden. Der Sterzinger Marmor mit ungleichkörnigem interlobatem Korngefüge weist ein entgegengesetztes Bild auf. Der in diesem Marmor erzeugte Zugbruch verläuft zumeist entlang intrakristalliner Flächen (ca. 81\%) und ist seltener an Korngrenzen gebunden (ungefähr $18 \%$; s. Abb. 4.26b).

Um zu überprüfen, auf welche Gefügemerkmale die festzustellenden Anisotropien der Spaltzugfestigkeiten ursächlich zurückzuführen sind, ist in Abb. 4.25, die bevorzugte Orientierung der Mikrorisse in Form von Ultraschallgeschwindigkeitsdifferenzen, die Textur und die Korngrenzenorientierung zu den Spaltzugfestigkeiten aufgetragen. Die $\Delta \mathrm{V}_{\mathrm{p}}$-Werte wurden aus der Differenz zwischen trockenem und wassergesättigtem Zustand (Kugelmessungen) in der entsprechenden Gefügerichtung ermittelt. Aus Gründen der Übersichtlichkeit wurden die $\Delta \mathrm{V}_{\mathrm{p}}$-Daten um $90^{\circ}$ gedreht. Damit geben die Ultraschallgeschwindigkeitsdifferenzen nicht mehr ihre Richtung wieder in der sie gemessen wurden, sondern das Streichen der Risse, die durch sie charakterisiert werden. Die bevorzugte Orientierung der Korngrenzen und die Textur (r- $\{104\}$ und e-Fläche $\{018\}$ ) sind ebenfalls im Streichen angegeben.

Generell besitzen offene Mikrorisse in Gesteinen den größten Einfluss auf die Spaltzugfestigkeit (vgl. PECK et al., 1985; BROSCH et al., 2000). Vorhandene wie auch durch den Spaltzugversuch erzeugte Mikrorisse wachsen im Allgemeinen entlang von Schwächezonen des Gesteins und bilden sich damit an Korngrenzen und an intragranularen Flächen aus. Daher soll der Einfluss von präexistierenden offenen Mikrorissen auf die Spaltzugfestigkeit im Folgenden zusammen mit den weiteren Gefügemerkmalen der einzelnen Proben diskutiert werden.

Für die deutliche Richtungsabhängigkeit der Spaltzugfestigkeit des Carrara Marmors sollte aufgrund der festgestellten bevorzugten Orientierung des Bruches an Korngrenzen der Einfluss der Kornformorientierung dominieren. Beim Vergleich von Abb. 4.25a mit Abb. 4.25c ist erkennbar, dass das bevorzugte Streichen der Korngrenzen mit geringen Festigkeitsdaten für den Zugbruch zwischen $45^{\circ}$ und $135^{\circ}$ weitgehend übereinstimmt. Aufgrund der äußerst schwachen Regelung der r- und e-Flächen kann hier ein fehlender oder nur sehr untergeordneter Einfluss der Textur angenommen werden (vgl. Abb. 4.25b). Aufgrund der Mikrobereichsanalysen, nach denen der Carrara Marmor auch verwitterungsbedingt fast ausschließlich an Korngrenzen entfestigt, ist davon auszugehen, dass die bevorzugte Orientierung von existierenden Mikrorissen die Kornform nachzeichnet. Damit sollte es sich bei der Richtungsabhängigkeit der Spaltzugfestigkeit um einen überlagernden Effekt aus Aktivierung bereits vorhandener nach, der Kornformorientierung 

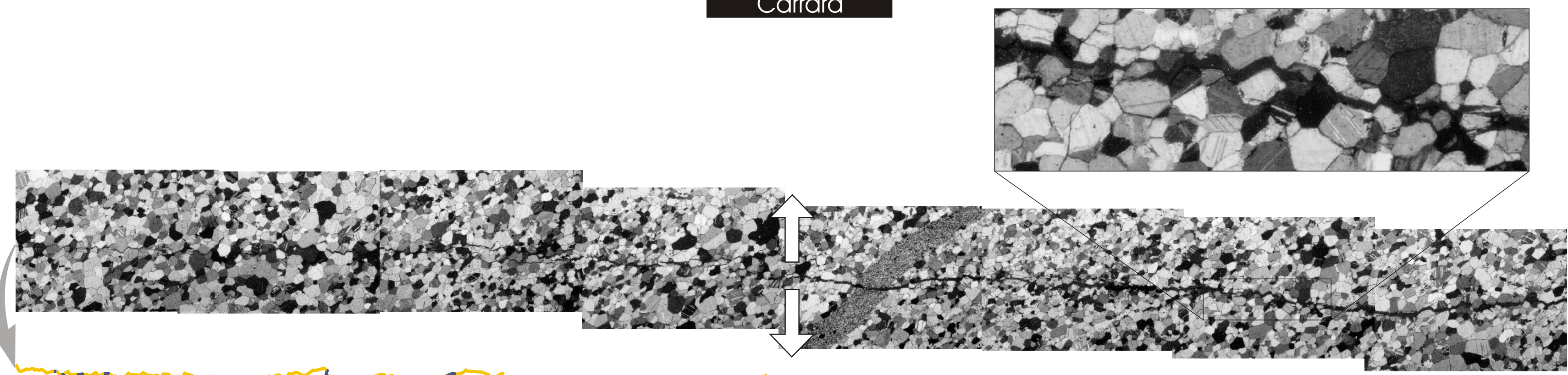

- intrakristallin ca. $17 \%$

— intergranular ca. 83\%

a)

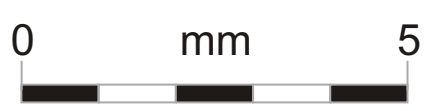

Sterzing

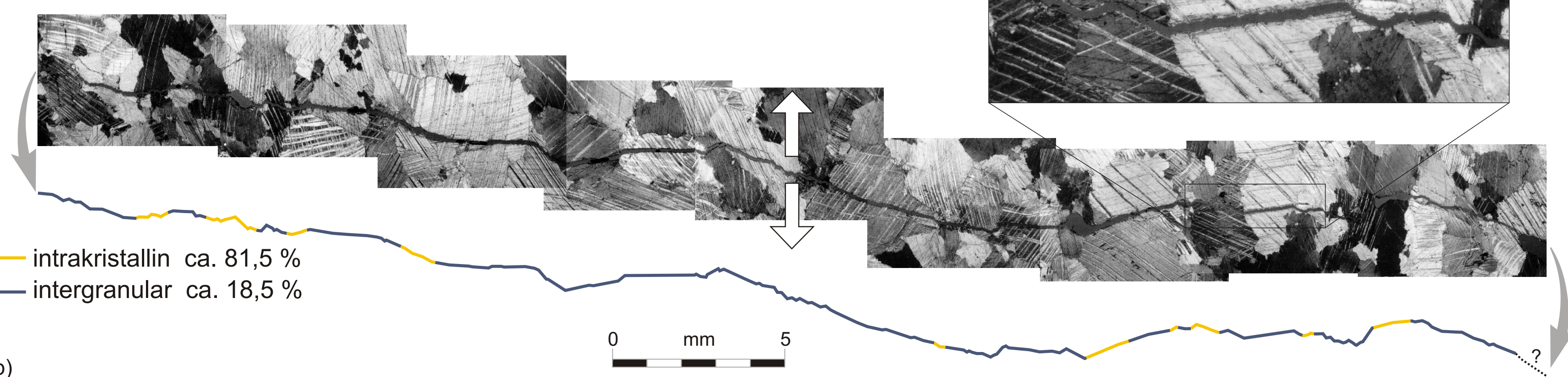

Abb. 4.26: Durch den Spaltzugversuch erzeugte Brüche ausgewählter Proben des a) Carrara und b) Sterzinger Marmors. 
ausgerichteter Mikrorisse und neugebildeter, ebenfalls der Kornformorientierung folgender Risse handeln.

Im Gegensatz zum Carrara Marmor sollte der Sterzinger Marmor einen deutlicheren Einfluss der bevorzugten Orientierung der Kristallflächen bezüglich der Spaltzugfestigkeit aufweisen, da zumeist intrakristalline Risse durch den Zugbruchversuch geöffnet wurden. Das bevorzugte Streichen der r- und e-Flächen stimmt hierbei mit einer Abnahme der Spaltzugfestigkeit überein. Beide Kristallflächen zeigen ein Maximum des bevorzugten Streichens zwischen $90^{\circ}$ und $120^{\circ}$ (s. Abb. 4.25f). In diesem Bereich weisen auch die erzwungenen Zugbrüche die geringsten Festigkeiten auf. Die bevorzugte Orientierung der Korngrenzen liegt ebenfalls im Bereich der niedrigsten Festigkeiten. Ihr Einfluss sollte jedoch aufgrund einer starken Verzahnung der Korngrenzen nur untergeordnet für die Anisotropie der Festigkeit von Bedeutung sein. Nach Mikrobereichsanalysen sind die präexistierenden Risse des Sterzinger Marmors zumeist entlang von intragranularen Flächen entwickelt. Daher ist auch beim Spaltzugversuch des Sterzinger Marmors davon auszugehen, dass es sich weitgehend um eine Überlagerung von verwitterungsbedingten intrakristallinen Rissen und durch die Beanspruchung neu gebildeter intrakristalliner Risse handelt.

Die Untersuchungen zeigen, dass das Gefügeinventar einen großen Einfluss auf die Spaltzugfestigkeiten hat. Insbesondere die durch die Kornformregelung erzeugte starke Festigkeitsanisotropie des Carrara Marmors zeigt, dass bezüglich von Untersuchungen zur Herabsetzung der Festigkeit durch Verwitterungsprozesse oder auch zur festigenden Wirkung von Konservierungsmitteln von Marmoren das Gefügeinventar und seine Richtungsabhängigkeit Berücksichtigung finden muss. 


\section{Gefügeabhängigkeit des thermischen Verwitterungsverhaltens von Marmoren}

Als entscheidender Prozess, der die Gefügeentfestigung in kristallinen Marmoren einleitet, wird die Mikrorissbildung durch thermische Beanspruchung angesehen. KESSLER (1919) beobachtete, dass wiederholtes Aufheizen von Marmor zu einer Restdehnung führt, d.h. dass sich das Gestein nach thermischer Beanspruchung nicht auf seine Ausgangslänge zurückzieht. Dabei kann schon während des ersten Beanspruchungszyklus eine erhebliche permanente Längenänderung auftreten (ZEZZA et al., 1985; SAGE, 1988; TsCHEGG et al., 1999; SiEgESMund et al., 2000b). BATTAGLiA et al. (1993) ermittelte selbst für relativ geringe Temperaturänderungen von $20^{\circ} \mathrm{C}$ auf $50^{\circ} \mathrm{C}$ ausgeprägte Restdehnungen bei Marmoren.

Bezüglich der thermischen Gefügeentfestigung von Calcit-Marmoren kommt den extrem anisotropen Eigenschaften des Calcit-Einkristalls eine entscheidende Bedeutung zu (EvANS, 1978; FREDRICH \& WONG, 1986). So variiert der Koeffizient der thermischen Ausdehnung $(\alpha)$ des Einkristalls von positiven Werten parallel zur kristallographischen c-Achse bis zu negativen Werten parallel zu den a-Achsen (vgl. Abb. 5.1 und Kap. 2.1). Das bedeutet, dass sich Calcitkristalle bei Erwärmung parallel zur c-Achse dehnen und gleichzeitig parallel zu den a-Achsen eine Kontraktion stattfindet.

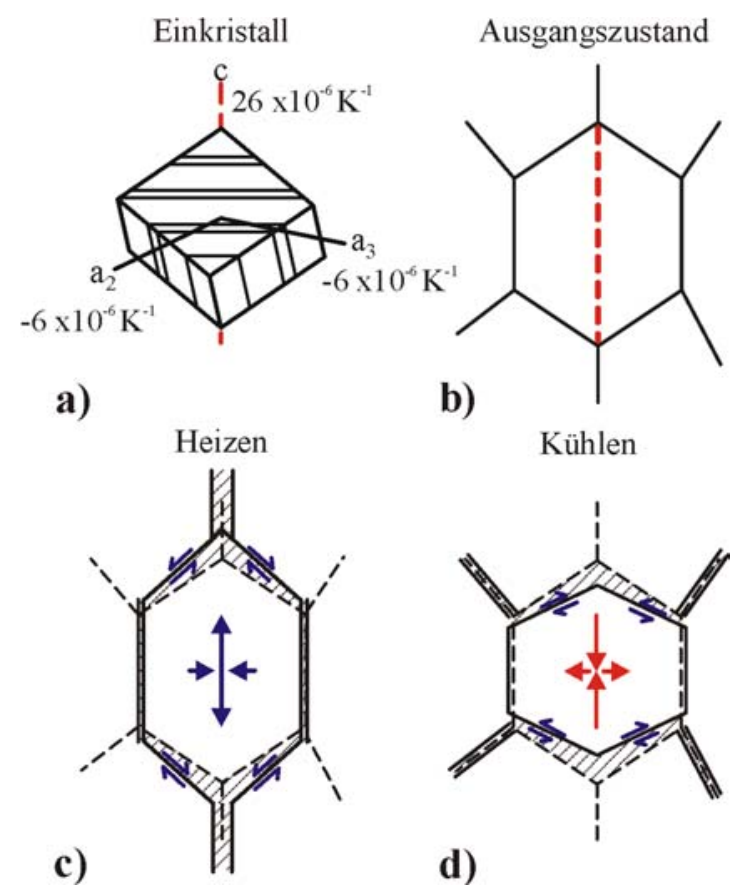

Abb. 5.1: Thermische Dilatation von Calcit: a) Thermische Ausdehnungskoeffizienten (KLEBER, 1959) des Calcit-Einkristalls in Richtung verschiedener kristallographischer Achsen, b) schematische Darstellung eines Calcit-Einkristalls bei Ausgangsbedingungen, c) Volumenänderung bei Erwärmung und d) bei Abkühlung. 


\subsection{Thermisches Längenänderungsverhalten}

Die in Marmoren zumeist ausgeprägten Texturen bedingen in Verbindung mit den anisotropen Einkristalleigenschaften eine Richtungsabhängigkeit der thermischen Längenänderung (SIEGESMUND et al., 2000c; RUEDRICH et al., 2001a). Daneben sind die Kristalleigenschaften für einen Spannungsaufbau bei Temperaturveränderung im Gestein maßgeblich, was zu einer Mikrorissbildung führen kann und in der permanenten Längenänderung nach einem thermischen Beanspruchungszyklus resultiert (SAGE, 1988; Battaglia, et al., 1993). Die Rissneubildung und eine Pufferung existierender Risse führt zu einem relativ hohen und mit Temperaturerhöhung stark ansteigenden Ausdehnungskoeffizienten des Gesamtgesteins.

\subsubsection{Anisotropie der thermischen Längenänderung von Marmor}

Bei Messungen der thermischen Dilatation ( $\varepsilon$ ) von Marmoren ist eine zumeist ausgeprägte Richtungsabhängigkeit der Längenänderung festzustellen (WIDHALM et al., 1996, TSCHEGG et al., 1999, SiEgeSmund et al., 2000c, RUEDRICH et al., 2001a). Dieses anisotrope Materialverhalten wird durch die Textur des jeweiligen Marmors bedingt. Aufgrund der bevorzugten Regelung der Kristalle können sich die anisotropen Dehnungseigenschaften des Calcit auf das Verhalten des Gesamtgesteins durchpausen. Dabei befindet sich die größte Dehnung parallel der bevorzugten Orientierung der c-Achsen und die geringste parallel der Regelung der a-Achsen. Je stärker die Regelung der Kristalle, also die Intensität der Textur ist, um so ausgeprägter ist die Anisotropie der Dehnung. Daneben können weitere Parameter die Richtungsabhängigkeit des Dehnungsverhaltens beeinflussen. Dies sind vor allem vorhandene sowie während der Beanspruchung wachsende Mikrorisse (vgl. Kap. 5.1.3).

Bei Idealtexturen folgt das Muster der anisotropen Dehnung bei Temperaturänderung den beiden Endgliedern der kristallographischen Vorzugsregelung, dem c- und dem aAchsenfasertyp (vgl. Kap. 2.2). Dabei ist für einen reinen c-Achsenfasertyp $\varepsilon_{1}>\varepsilon_{2}=\varepsilon_{3} \mathrm{zu}$ erwarten, wohingegen der a-Achsenfasertyp $\varepsilon_{1}=\varepsilon_{2}>\varepsilon_{3}$ zeigen sollte. Da Realtexturen zumeist eine Modifizierung des Verteilungsmusters durch Submaxima und -minima aufweisen, ist in der Regel $\varepsilon_{1}>\varepsilon_{2}>\varepsilon_{3}$ zu ermitteln (s. Abb. 5.2; vgl. LeISS \& UlLEMEYER, 1999).
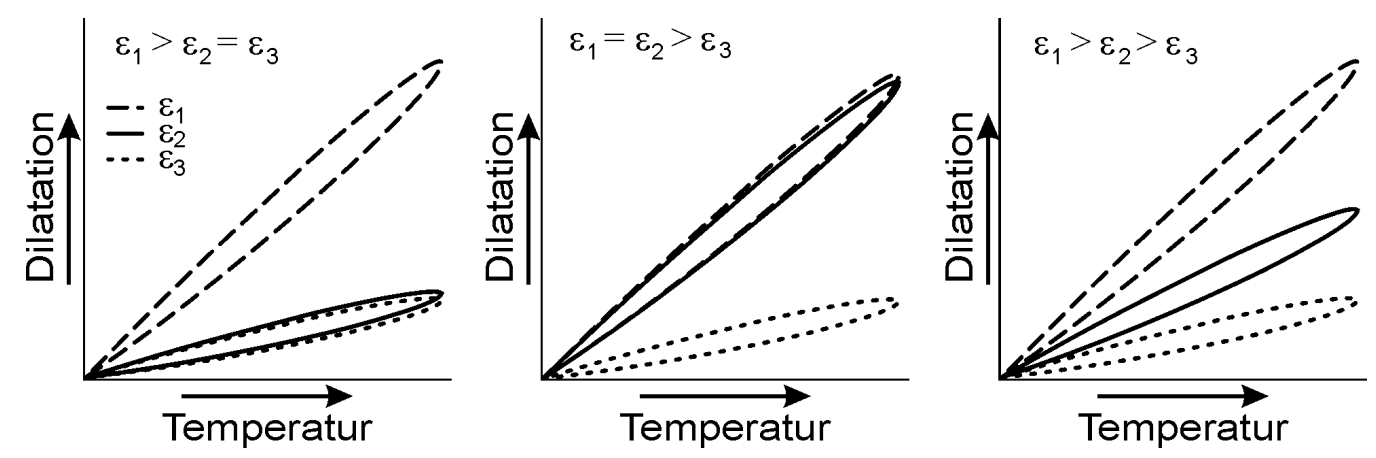

Abb. 5.2: Schematische Darstellung der Längenänderungen in unterschiedliche Richtungen von Marmoren hervorgerufenen durch verschiedene Texturmuster (Erläuterungen s. Text). 
Für die untersuchten Gesteine ist die Abhängigkeit des thermischen Dehnungsverhaltens von der Textur in Abb. 5.3 exemplarisch für den Sterzinger und Lasa Marmor bei Erwärmung auf $90{ }^{\circ} \mathrm{C}$ dargestellt. Dabei repräsentiert die Probe aus Sterzinger Marmor einen durch Submaxima modifizierten c-Achsenfasertyp, d.h. die c-Achsen weisen ein Punktmaximum auf und die a-Achsen sind durch eine unregelmäßige Gürtelverteilung gekennzeichnet (s. Abb. 5.3b). Die größte Dehnung ist hier parallel zum c-Achsenmaximum in Z-Richtung festzustellen, wobei in die X- und Y-Richtung und damit senkrecht zur c-Achsenfaser $\varepsilon$ deutlich geringer ist (Abb. 5.3a). Das Dehnungsverhalten der $\mathrm{X}$ - und Y-Richtungen ist aufgrund des modifizierten Texturmusters ungleich ausgebildet $\left(\varepsilon_{2}>\varepsilon_{3}\right)$.

Der Lasa Marmor repräsentiert hingegen einen a-Achsen Fasertyp, d.h. die c-Achsen weisen eine Gürtelverteilung auf und die Achsen formen ein Punktmaximum (Abb. 5.3d). Dies drückt sich im Dehnungsverhalten dadurch aus, dass parallel zur Z- und Y-Richtung ein weitgehend identisches Dehnungsverhalten festzustellen ist $\left(\varepsilon_{1}=\varepsilon_{2}\right)$. Die X-Richtung parallel zur a-Achsenfaser weist die geringste Dehnung auf (Abb. 5.3c).
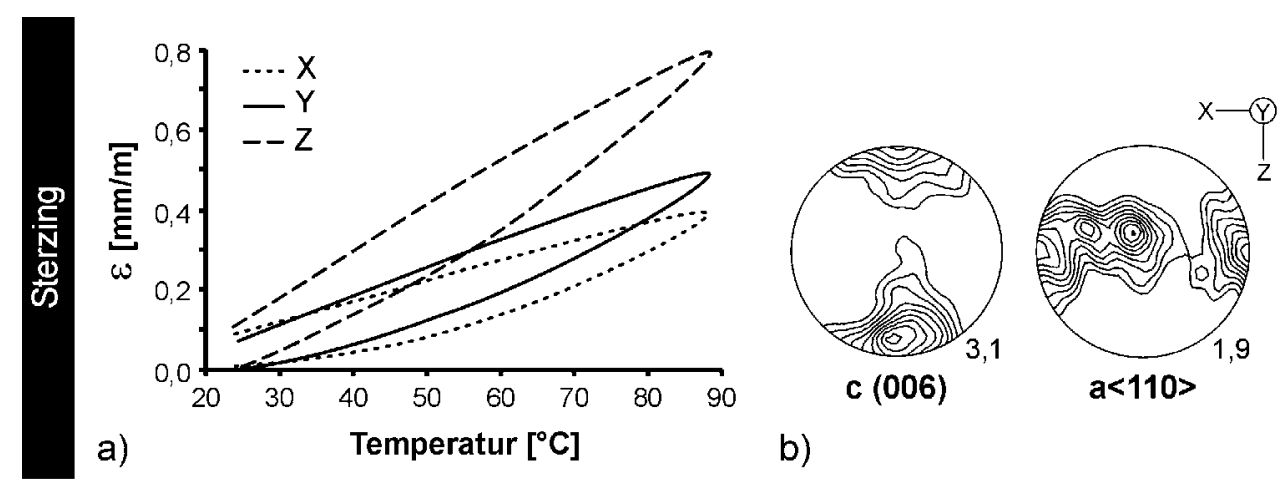

b)
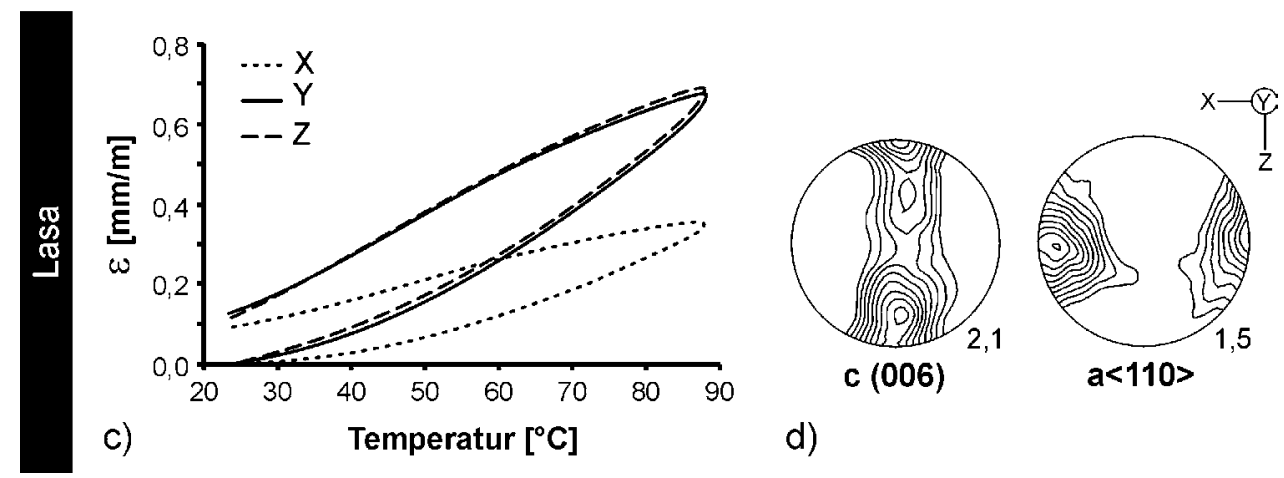

Abb. 5.3: Richtungsabhängigkeit der thermischen Dilatation $(\varepsilon)$, bedingt durch das Muster und die Intensität der Textur, für den Sterzinger und den Lasa Marmor: a,c) Dehnung als Funktion der Temperatur und b,d) Darstellung der Textur für die c- und a-Achsenverteilung (Intensität in $\mathrm{VGV}=$ Vielfaches der Gleichverteilung).

Die experimentell ermittelten richtungsabhängigen Längenänderungen für die Aufheizphasen von $65^{\circ} \mathrm{C}$ und $90{ }^{\circ} \mathrm{C}$ ausgewählter Marmorproben sind in Abb. 5.4a in Form von Ausdehnungskoeffizienten $(\alpha)$ dargestellt. Dabei variieren die $\alpha$-Werte zwischen $-1 \cdot 10^{-6} \mathrm{~K}^{-1}$ und $22 \cdot 10^{-6} \mathrm{~K}^{-1}$. Damit ist zum einen eine große Variabilität der Ausdehnungskoeffizienten zu ermitteln. Zum anderen kann festgestellt werden, dass Marmore bei Erwärmung auch in bestimmte Gefügerichtungen kontrahieren können (vgl. SIEGESMUND et al., 1999 ). Die in Abb. 5.4a dargestellten Balken werden von den minimalen und maximalen $\alpha$-Werten gebildet 
und repräsentieren damit die Anisotropie der Ausdehnungskoeffizienten des jeweiligen Marmors. Deutlich sind dabei die Unterschiede zwischen schwach und stark texturierten Marmoren, wie z.B. Carrara und Prieborner Marmor erkennbar. Weiterhin auffallend sind die generell deutlich erhöhten Ausdehnungskoeffizienten des $90{ }^{\circ} \mathrm{C}$ Heizzyklus gegenüber dem auf $65^{\circ} \mathrm{C}$. Die Zunahme der $\alpha$-Werte ist auf eine verstärkte Rissbildung in diesem Temperaturbereich zurückzuführen.

Um die Ausdehnungskoeffizienten zu veranschaulichen und in eine greifbare Messgröße zu überführen sind in Abb. 5.4b, auf der Basis der Calcit-Einkristalldaten nach Kleber (1959), berechnete Dehnungen eines realen Marmorkörpers mit $1 \mathrm{~m}$ Länge für spezifische Temperaturdifferenzen eingetragen. Sind die Ausdehnungskoeffizienten eines CalcitMarmors bekannt, kann die resultierende Dehnung für verschiedene thermische Beanspruchungen direkt aus dem Diagramm abgelesen werden. Dies ist anhand eines Säulenabschnittes für die experimentell ermittelten Dehnungsdaten des Prieborner Marmors für eine Temperaturdifferenz von $\Delta \mathrm{T}=40^{\circ} \mathrm{C}$ in Abb. $5.4 \mathrm{c}$ exemplarisch dargestellt.
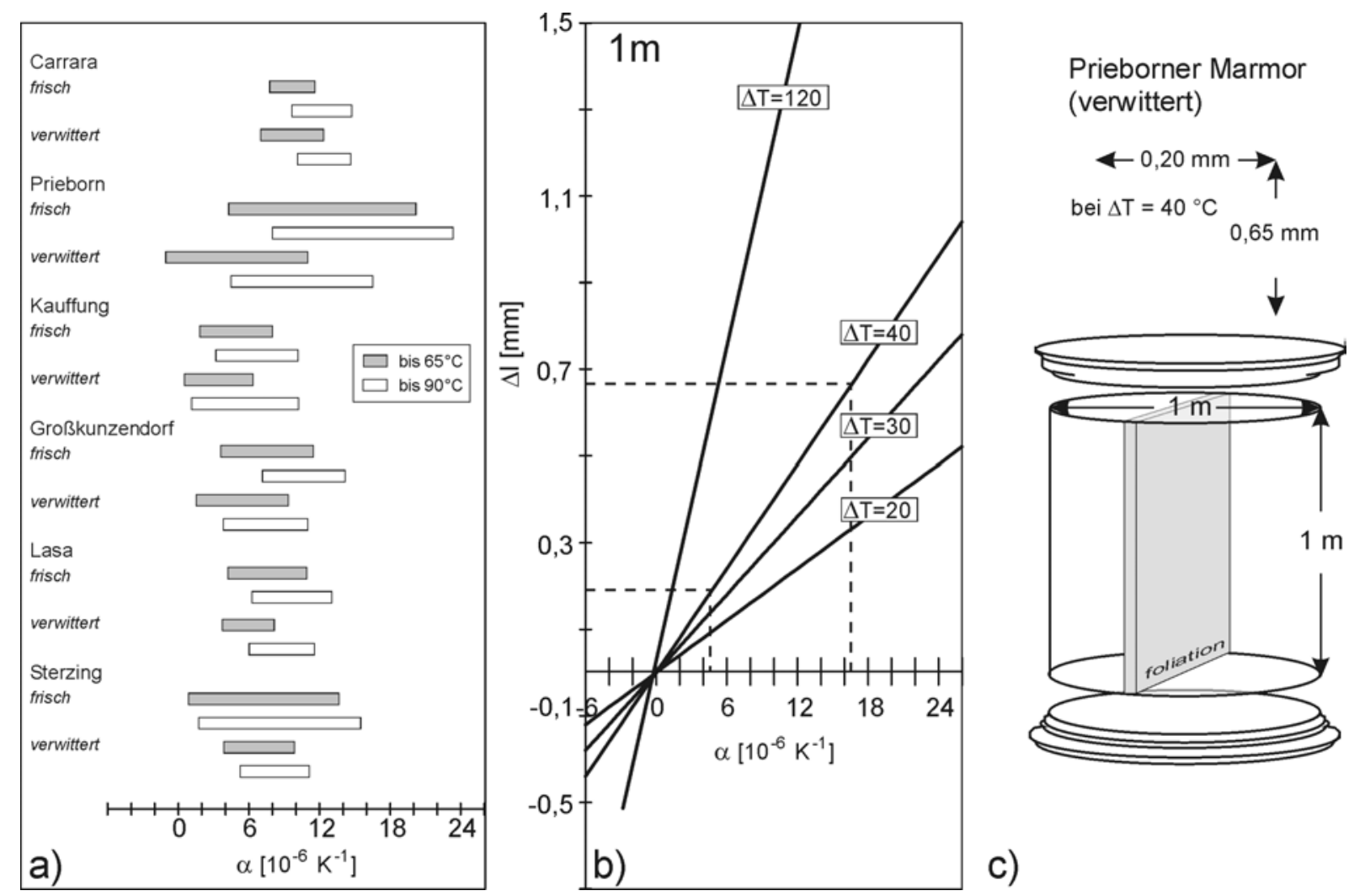

Abb. 5.4: Thermische Dehnung von Marmor: a) Experimentell ermittelte Ausdehnungskoeffizienten $\alpha$ der untersuchten Marmore. b) Auf der Basis der Calcit-Einkristalldaten von Kleber (1959) berechnete Dehnung einer Marmorplatte mit einer Länge von $1 \mathrm{~m}$. Wenn $\alpha$ eines bestimmten Marmors bekannt ist, kann die resultierende Dehnung für bestimmte Temperaturstufen direkt in b) abgelesen werden, was als Beispiel in c) für einen Säulenausschnitt für den verwitterten Prieborner Marmor dargestellt ist. Die jeweiligen Ausdehnungskoeffizienten wurden a) entnommen und sind in b) als gestrichelte Linien eingezeichnet.

Zur Veranschaulichung wurden die experimentell ermittelten Ausdehnungskoeffizienten des Heizzyklus auf $90^{\circ} \mathrm{C}$ gewählt, welche minimal $4,5 \cdot 10^{-6} \mathrm{~K}^{-1}$ und maximal $16,5 \cdot 10^{-6} \mathrm{~K}^{-1}$ 
betragen. Bei Überführung der Daten, wie in Abb. 5.4b durch eine gestrichelte Linie dargestellt, liegt die Dehnung senkrecht zur Foliation und damit parallel zu den c-Achsen bei $0,6 \mathrm{~mm} / \mathrm{m}$, womit diese das Dreifache dessen beträgt, was senkrecht zur Foliation, also parallel zum a-Achsenmaximum, zu ermitteln ist. Der deutliche Dehnungskontrast für die unterschiedlichen Richtungen zeigt, dass die Kenntnis der Dehnungseigenschaften insbesondere bei stark anisotropen Gesteinen auch bautechnische Relevanz besitzen kann, z.B. für materialangepasste Ankersysteme oder die Orientierung von Fierungen.

Die ausgeprägte Richtungsabhängigkeit der thermischen Längenänderung von Marmoren ist ebenfalls für die Abkühlung des Materials festzustellen. Dies ist in Abb. 5.5 exemplarisch für die verwitterte Probe aus Prieborner Marmor dargestellt. Auffällig ist die deutliche Dehnung des Materials parallel zur X-Richtung bei Absenkung der Temperatur. Hierbei paust sich die bevorzugte Orientierung der a-Achsen in Verbindung mit den anisotropen CalcitEigenschaften sehr deutlich auf das Gesamtgesteinsverhalten durch. Die stark texturierte Prieborner Probe zeigt, dass sich Marmore bei Abkühlung auch in spezifische Gefügerichtungen dehnen können.

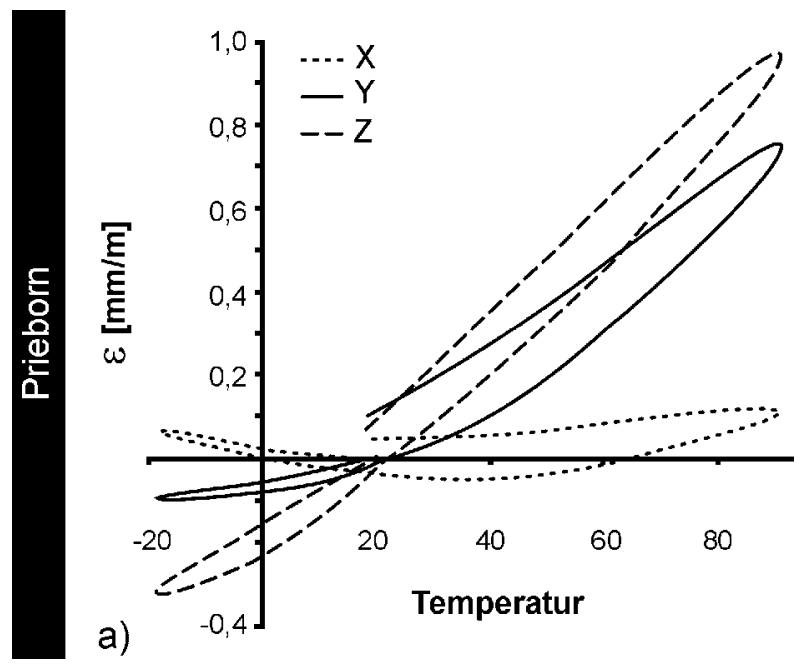

Abb. 5.5: Experimentell ermitteltes Dehnungsverhalten der verwitterten Probe aus Prieborner Marmor bei thermischen Beanspruchungszyklen auf $-20^{\circ} \mathrm{C}$ und folgend auf $90{ }^{\circ} \mathrm{C}$ (Ausgangs- und Haltetemperatur $20^{\circ} \mathrm{C}$ ).

\subsubsection{Restdehnung (Thermische Mikrorissbildung)}

Marmore, die einer thermischen Beanspruchung ausgesetzt werden, zeigen bei Erreichen der Ausgangstemperatur zumeist eine bleibende Längenänderung. Diese Restdehnung wird durch das Aufreißen neuer und durch die Weitung vorhandener offener Mikrorisse hervorgerufen (vgl. SAGE, 1988). Bei Temperaturwechseln können sich aufgrund des anisotropen Dehnungsverhaltens des Calcit-Einkristalls im Korngefüge Zug-, Druck- oder Scherspannungen aufbauen, die zum Aufreißen von inter- und intrakristallinen Flächen führen (s. Abb. 5.6a; Battaglia et al., 1993). Das Risswachstum findet dabei im Aufheizstadium statt, was anhand der stark ansteigenden Dehnung und der Zunahme akustischer Emissionen in dieser Phase ermittelt werden kann (s. Abb. 5.6b; vgl. WidHALM et al., 1999). Die Längenänderung, welche nach Beanspruchung eines Marmors auf eine bestimmte Temperatur 
zurückbleibt, kann als Grad der Schädigung und somit auch als Maß seiner thermischen Sensibilität gewertet werden.

Die permanente Längenänderung kann sowohl für den jeweiligen Marmor als auch bezüglich seiner Richtungsabhängigkeit sehr unterschiedlich sein. Die in Tab. 5.1 angegebenen Werte der Restdehnung $(\Delta \varepsilon)$ für ausgewählte unverwitterte und verwitterte Marmorproben geben einen Überblick über die experimentell ermittelten Daten.

Für geringe thermische Beanspruchung auf $42{ }^{\circ} \mathrm{C}$ sind keine oder nur geringe Restdehnungsbeträge für die untersuchten Marmore festzustellen. Hingegen sind sehr hohe permanente Längenänderungsbeträge für die Heizzyklen auf $65^{\circ} \mathrm{C}$ und $90{ }^{\circ} \mathrm{C} \mathrm{zu}$ ermitteln. Hierbei liegen die höchsten $\Delta \varepsilon$-Werte um $0,150 \mathrm{~mm} / \mathrm{m}$. Die größten Restdehnungen treten dabei vorwiegend bei unverwitterten Marmoren auf. Eine Ausnahme stellt dabei der verwitterte Prieborner Marmor dar. Er zeigt mit $0,134 \mathrm{~mm} / \mathrm{m}$ bei Temperaturerhöhung auf $90{ }^{\circ} \mathrm{C}$ deutlich höhere $\Delta \varepsilon$-Werte sowohl gegenüber den weiteren verwitterten Proben als auch gegenüber einigen frischen Marmoren. Auffallend ist weiterhin das thermische Verhalten der stark verwitterten Probe aus Carrara Marmor. Parallel zur Z-Richtung ist für alle Temperaturstufen eine ausgeprägte Kontraktion $\mathrm{zu}$ ermitteln. Hier ist von einem „Gefügekollaps“ der, möglicherweise zusätzlich durch die Präparation, stark aufgelockerten Probe auszugehen (vgl. RUEDRICH et al., 2002).
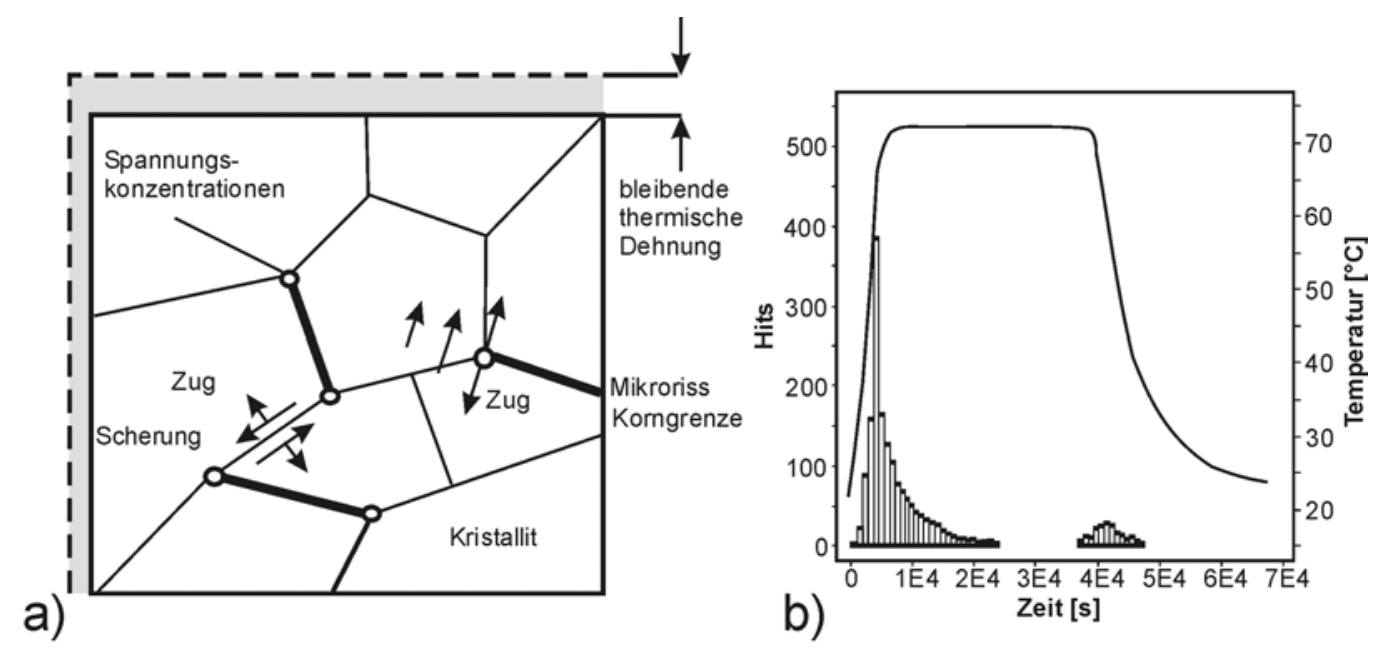

Abb. 5.6: a) Schematische Darstellung der Entstehung von Rissen in Marmoren bei thermischer Beanspruchung (aus SAGE, 1988) und b) Anzahl der akustischen Emissionen einer Carrara Marmorprobe bei thermischer Beanspruchung auf ca. $70^{\circ} \mathrm{C}$.

Für den zweiten Heizzyklus auf $90{ }^{\circ} \mathrm{C}$ sind für die Proben im Allgemeinen keine weiteren Restdehnungsbeträge zu ermitteln. Eine Ausnahme stellt dabei die frische Probe des Prieborner Marmors dar. Bezüglich des Verwitterungszustandes der untersuchten Marmore sind Unterschiede des Risswachstumsmechanismus anzunehmen. Hierbei ist davon auszugehen, dass bezüglich der Restdehnungen der frischen Marmore vorwiegend eine Rissneubildung während der thermischen Beanspruchung stattfindet. Hingegen zeigen die verwitterten Marmore nach Mikrobereichsanalysen ein gesteinsdurchdringendes Risssystem schon vor der Beanspruchung. Hier sollte vor allem eine Weitung vorhandener Risse bei Wärmebehandlung erfolgen. 


\section{- Anisotropie der Restdehnung und ihre Abhängigkeit vom Gefüge}

In den experimentellen Dehnungsmessungen kann festgestellt werden, dass neben der absoluten Dehnung auch die Restdehnung nach einem thermischen Beanspruchungszyklus eine Richtungsabhängigkeit aufweist (vgl. Tab. 5.1).

Tab. 5.1: Experimentell ermittelte Restdehnungen $(\Delta \varepsilon)$ in die Hauptgefügerichtungen für die Heizzyklen $42{ }^{\circ} \mathrm{C}, 65^{\circ} \mathrm{C}, 90{ }^{\circ} \mathrm{C}$ und wiederholtem Heizzyklus auf $90{ }^{\circ} \mathrm{C}$ frischer und verwitterter Marmore. Werte unter $0,020 \mathrm{~mm} / \mathrm{m}$ wurden gleich null gesetzt (unterhalb der gerätetechnischen Auflösung) und Werte über $0,050 \mathrm{~mm} / \mathrm{m}$ sind fett gedruckt (ausgeprägte Restdehnung; n.d. = nicht detektierbar).

\begin{tabular}{|c|c|c|c|c|c|c|c|}
\hline Marmortyp & Zustand & Richtung & $\begin{array}{c}1 \text { Zyklus } \\
42{ }^{\circ} \mathrm{C} \\
\Delta \varepsilon \\
{[\mathrm{mm} / \mathrm{m}]}\end{array}$ & $\begin{array}{c}2 \text { Zyklus } \\
65^{\circ} \mathrm{C} \\
\Delta \varepsilon \\
{[\mathrm{mm} / \mathrm{m}]}\end{array}$ & $\begin{array}{c}3 \text { Zyklus } \\
90^{\circ} \mathrm{C} \\
\Delta \varepsilon \\
{[\mathrm{mm} / \mathrm{m}]}\end{array}$ & $\begin{array}{c}4 \text { Zyklus } \\
90^{\circ} \mathrm{C} \\
\Delta \varepsilon \\
{[\mathrm{mm} / \mathrm{m}]}\end{array}$ & $\begin{array}{c}\text { Gesamt } \\
\Delta \varepsilon \\
{[\mathrm{mm} / \mathrm{m}]}\end{array}$ \\
\hline \multirow{9}{*}{ Carrara } & \multirow{3}{*}{ frisch } & $\mathrm{X}$ & 0,025 & 0,046 & 0,065 & 0,000 & 0,136 \\
\hline & & Y & 0,040 & 0,066 & 0,110 & 0,000 & 0,216 \\
\hline & & Z & 0,041 & 0,060 & 0,120 & 0,023 & 0,244 \\
\hline & \multirow{3}{*}{ verwittert } & $\mathrm{X}$ & 0,029 & 0,039 & 0,112 & 0,000 & 0,180 \\
\hline & & Y & 0,000 & 0,042 & 0,109 & 0,022 & 0,173 \\
\hline & & Z & 0,000 & 0,000 & 0,054 & 0,000 & 0,054 \\
\hline & \multirow{3}{*}{$\begin{array}{c}\text { stark } \\
\text { verwittert }\end{array}$} & $\mathrm{X}$ & 0,000 & 0,000 & 0,000 & 0,000 & 0,000 \\
\hline & & Y & 0,000 & 0,000 & 0,000 & 0,000 & 0,000 \\
\hline & & Z & $-0,071$ & $-0,047$ & $-0,055$ & $-0,032$ & $-0,205$ \\
\hline \multirow{6}{*}{ Prieborn } & \multirow{3}{*}{ frisch } & $\mathrm{X}$ & 0,000 & 0,029 & 0,148 & 0,035 & 0,212 \\
\hline & & Y & 0,026 & 0,026 & 0,148 & 0,034 & 0,234 \\
\hline & & Z & 0,035 & 0,040 & 0,154 & 0,030 & 0,259 \\
\hline & \multirow{3}{*}{ verwittert } & $\mathrm{X}$ & 0,000 & $\mathbf{0 , 0 5 4}$ & 0,099 & 0,000 & 0,153 \\
\hline & & Y & 0,027 & 0,086 & 0,134 & 0,000 & 0,247 \\
\hline & & Z & 0,042 & 0,066 & 0,094 & 0,000 & 0,202 \\
\hline \multirow{6}{*}{ Kauffung } & \multirow{3}{*}{ frisch } & $\mathrm{X}$ & 0,000 & 0,016 & 0,014 & 0,000 & 0,030 \\
\hline & & Y & 0,000 & 0,016 & 0,014 & 0,000 & 0,030 \\
\hline & & Z & 0,024 & 0,030 & 0,027 & 0,000 & 0,081 \\
\hline & \multirow{3}{*}{ verwittert } & $\mathrm{X}$ & 0,000 & 0,000 & 0,000 & 0,000 & 0,000 \\
\hline & & Y & 0,000 & 0,000 & 0,000 & 0,000 & 0,000 \\
\hline & & Z & 0,000 & 0,000 & 0,000 & 0,000 & 0,000 \\
\hline \multirow{6}{*}{ Grosskunzendorf } & \multirow{3}{*}{ frisch } & $\mathrm{X}$ & 0,021 & 0,054 & 0,071 & 0,000 & 0,146 \\
\hline & & Y & 0,041 & 0,073 & 0,086 & 0,000 & 0,200 \\
\hline & & $\mathrm{Z}$ & 0,026 & 0,047 & 0,065 & 0,000 & 0,138 \\
\hline & \multirow{3}{*}{ verwittert } & $\mathrm{X}$ & 0,000 & 0,020 & 0,000 & 0,000 & 0,020 \\
\hline & & $\mathrm{Y}$ & 0,000 & 0,000 & 0,000 & 0,000 & 0,000 \\
\hline & & Z & n.d. & n.d. & n.d. & n.d. & n.d. \\
\hline \multirow{6}{*}{ Lasa } & \multirow{3}{*}{ frisch } & $\mathrm{X}$ & 0,024 & 0,061 & 0,101 & 0,000 & 0,186 \\
\hline & & Y & 0,000 & 0,052 & 0,094 & 0,000 & 0,146 \\
\hline & & Z & 0,022 & 0,069 & 0,120 & 0,000 & 0,211 \\
\hline & \multirow{3}{*}{ verwittert } & $\mathrm{X}$ & 0,000 & 0,033 & 0,074 & 0,000 & 0,107 \\
\hline & & Y & 0,000 & 0,023 & 0,060 & 0,000 & 0,083 \\
\hline & & Z & 0,000 & 0,033 & 0,064 & 0,000 & 0,097 \\
\hline \multirow{6}{*}{ Sterzing } & \multirow{3}{*}{ frisch } & $\mathrm{X}$ & 0,000 & 0,000 & 0,047 & 0,000 & 0,047 \\
\hline & & Y & 0,000 & 0,000 & 0,074 & 0,000 & 0,074 \\
\hline & & $\mathrm{Z}$ & 0,071 & 0,033 & 0,127 & 0,000 & 0,231 \\
\hline & \multirow{3}{*}{ verwittert } & $\mathrm{X}$ & 0,000 & 0,000 & 0,049 & 0,000 & 0,049 \\
\hline & & Y & 0,000 & 0,026 & 0,056 & 0,000 & 0,082 \\
\hline & & Z & 0,035 & 0,026 & 0,054 & 0,000 & 0,115 \\
\hline
\end{tabular}


Für die untersuchten Proben ist in der Mehrzahl der Fälle festzustellen, dass die größte permanente Längenänderung der thermisch belasteten Probe in der jeweiligen Richtung auftritt, in welcher auch die stärkste Dehnung zu beobachten ist. Entsprechend ist das Verhalten in den weiteren Gefügerichtungen (s. Abb. 5.3). Daraus resultiert, dass Risse senkrecht zur bevorzugten Orientierung der c-Achsen am häufigsten gebildet und/oder am stärksten geweitet werden. In einigen Fällen ist jedoch auch ein abweichendes Verhalten der permanenten Längenänderung zu ermitteln. Dies ist z.B. für den Prieborner Marmor der Fall.

Die größte Restdehnung tritt hier parallel zur Y-Richtung auf, welche von der maximalen Ausdehnung eine intermediäre Richtung darstellt (Abb. 5.7). Der Prieborner Marmor weist eine bevorzugte Orientierung der Korngrenzen auf, welche sich parallel zur XZ-Ebene und damit senkrecht zur Y-Richtung befindet (Abb. 5.7c). Aufgrund der reinen Korngrenzenentfestigung dieses Marmors ist davon auszugehen, dass die bevorzugte Orientierung der Korngrenzen zu einer höheren Rissdichte senkrecht zur Y-Richtung führt und somit für die größere Restdehnung verantwortlich ist.
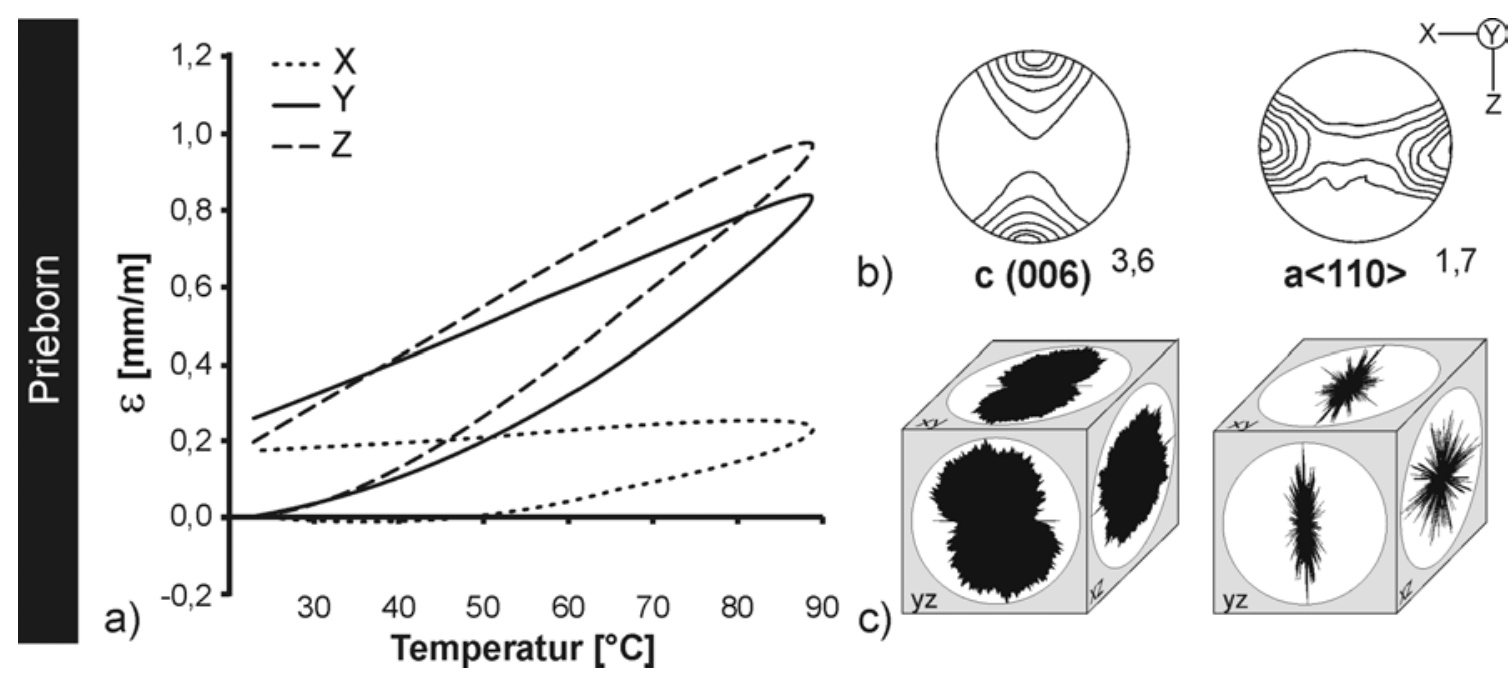

c)
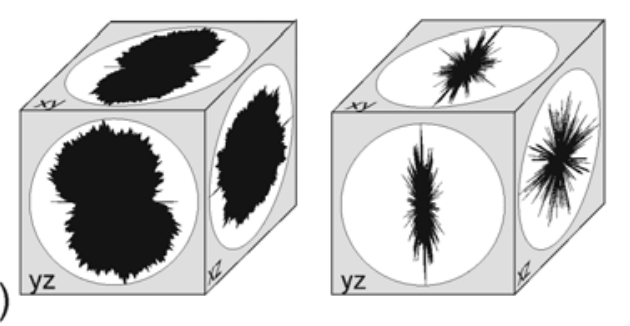

Abb. 5.7: Abhängigkeit der anisotropen Restdehnung von Textur und Kornformregelung für den Prieborner Marmor: a) thermische Dehnung, b) Textur (Intensität in VGV = Vielfaches der Gleichverteilung) und c) Korngrenzenorientierung (links) sowie Kornlangachsenregelung (rechts).

Generell ergibt sich die permanente Längenänderung aus der Rissdichte, also der Häufigkeit von Rissen sowie aus der jeweiligen Rissweite, d.h. dem Grad der Öffnung der Mikrobrüche. Die Richtungsabhängigkeit der Restdehnung ist somit auf anisotrope Verteilungsmuster von Rissdichte und/oder Rissweiten zurückzuführen. Je nachdem wie sich Rissdichte und Rissweiten überlagern, können isotrope und anisotrope Restdehnungen resultieren (s. Abb. 5.8). Eine anisotrope Rissdichte kann durch Aktivierung von gerichteten Schwachstellen im Gestein hervorgerufen werden, z.B. eine bevorzugte Orientierung von Korngrenzen. Hingegen ist eine anisotrope Rissweitung im Wesentlichen auf Unterschiede in den Dehnungsraten der verschiedenen Richtungen zurückzuführen (z.B. hohe Ausdehnung parallel zur c-Achsen Regelung). 

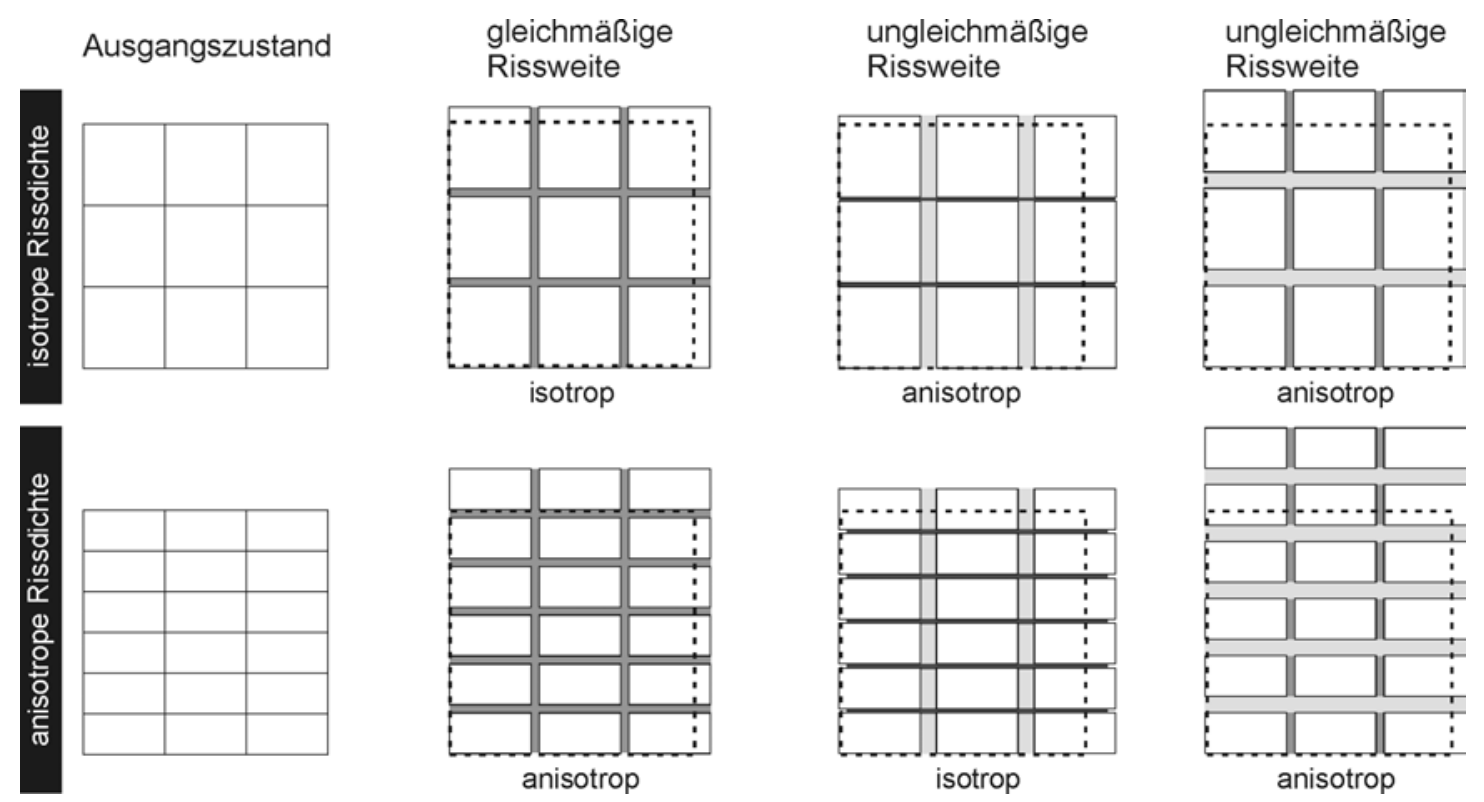

Abb. 5.8: Schematische Darstellung der Überlagerung isotroper und anisotroper Verteilungen von Rissdichten und Rissweiten.

Ein Einfluss der bevorzugten Orientierung von Korngrenzen auf das Restdehnungsverhalten, kann jedoch nur dann eine entscheidende Bedeutung besitzen, wenn der wesentliche Mechanismus die Korngrenzenentfestigung ist. Dies wird für equigranulare polygonale Marmore des Korngefügetyps I von Belang sein. Für grobkörnige Marmore mit verzahnten Korngrenzen (Korngefügetyp II), die eine umfangreiche Rissbildung an intrakristallinen Flächen aufweisen, sollte die Korngrenzenregelung eine geringere Rolle bezüglich der anisotropen Restdehnung spielen. Vielmehr können hier andere Gesteinsdiskontinuitäten, wie z.B. die bevorzugte Orientierung von Zwillingslamellen, welche wiederum Textur kontrolliert ist, dominierenden Einfluss besitzen.

\section{- Intensität der Restdehnung und ihre Gefügeabhängigkeit}

Die Intensität der Restdehnung eines Marmors ist charakteristisch für seine Sensibilität gegenüber thermischer Beanspruchung. Dabei ist eine entscheidende Frage, auch hinsichtlich der praktischen Anwendung, ob für eine besondere Anfälligkeit der thermischen Rissbildung einzelne Gefügemerkmale verantwortlich sind. Der Vergleich der Restdehnung mit einzelnen Mikrogesteinsmerkmalen zeigt jedoch, dass die Intensität der Restdehnung nicht auf ein einzelnes Gefügeelement wie z.B. die Korngröße zurückgeführt werden kann. Es handelt sich vielmehr um ein komplexes Zusammenspiel verschiedener Gefügeparameter (vgl. ZEISIG et al., 2002).

In Abb. 5.9 ist die Abhängigkeit der Restdehnung in Bezug auf die verschiedenen Korngefügetypen exemplarisch für die verwitterten Proben des Carrara, des Sterzinger und des Kauffunger Marmors dargestellt. Der Carrara (Typ I) und auch der Sterzinger Marmor (Typ II) weisen bei thermischer Beanspruchung auf $90{ }^{\circ} \mathrm{C}$ eine ausgeprägte Restdehnung auf, obwohl sie sich anhand des Korngefüges deutlich unterscheiden. 

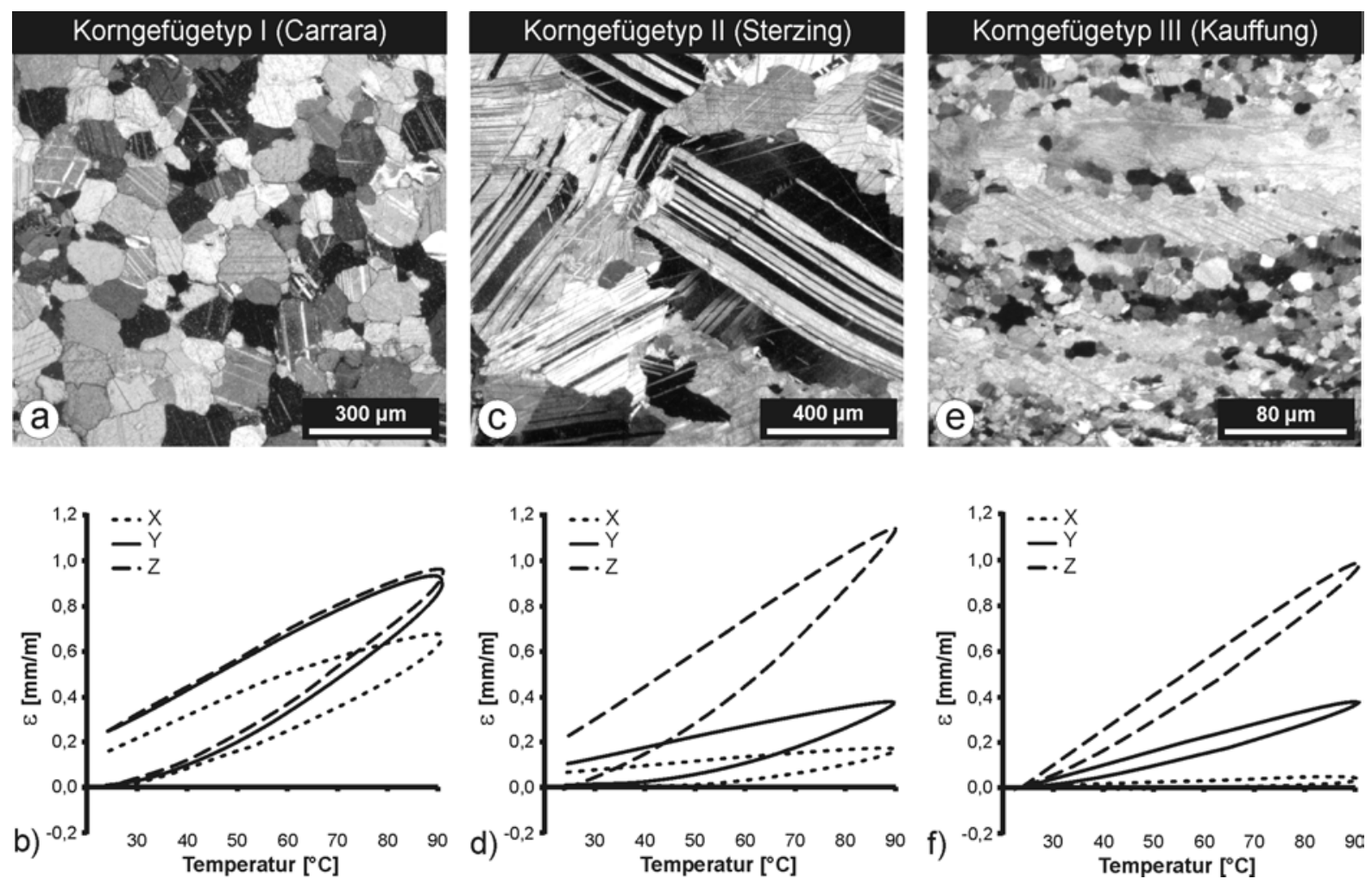

Abb. 5.9: Mikrobereichsaufnahmen (POL) und die zugehörige experimentell bestimmte thermische Dilatation als Funktion der Temperatur bis $90^{\circ} \mathrm{C}:(\mathrm{a}, \mathrm{b})$ Carrara Marmortyp mit equilibrierten Korngrenzen und (c, d) Großkunzendorfer Marmor mit interlobaten Korngrenzen sowie (e, f) Kauffunger Marmor. Auffallend ist die erhebliche Restdehnung der Carrara Probe im Gegensatz zur Kauffunger Marmor Probe welche praktisch keine Schädigung bei thermischer Beanspruchung aufweist.

Die in Tab. 5.1 angegebenen permanenten Längenänderungen belegen zwar zumeist leicht erhöhte $\Delta \varepsilon$-Werte für die Marmore des Korngefügetyps I gegenüber dem des Typs II. Die Unterschiede sind jedoch $\mathrm{zu}$ gering um einen eindeutigen Zusammenhang zwischen Korngrenzengeometrie bzw. Kornform und der Intensität der Restdehnung nachzuweisen. Der Kauffunger Marmor (Typ III) zeigt hingegen bei gleicher thermischer Beanspruchung keine Restdehnung, obwohl insbesondere die feinkörnig rekristallisierten Bereiche häufig polygonale Kornformen aufweisen, welche eine Korngrenzenentfestigung begünstigen.

Ein Vergleich der Restdehnungsbeträge mit der Korngröße der untersuchten Marmore ist in Abb. 5.10a dargestellt. Danach weisen Marmore, die durch mittlere Korngrößen oberhalb von $150 \mu \mathrm{m}$ gekennzeichnet sind, relativ hohe Restdehnungsbeträge auf. Für die grobkörnigeren Marmore ist lediglich eine schwache Tendenz zu niedrigeren permanenten Längenänderungen zu beobachten. Auffällig ist, dass die beiden Proben des Kauffunger Marmors mit einer mittleren Korngröße von unterhalb $50 \mu \mathrm{m}$ nur geringe bzw. fehlende permanente Längenänderungen anzeigen. Die schwachen Restdehnungsbeträge des Kauffunger Marmors sind jedoch nicht zwangsläufig nur auf die Korngröße zurückzuführen, da das Gefüge sich in vieler Hinsicht von dem der anderen Marmore unterscheidet. Weiterhin ist ebenso keine Abhängigkeit der absoluten Restdehnungsbeträge von der Textur zu ermitteln (vgl. Abb. 5.10b). Sowohl für die stark als auch für die schwach texturierten Marmore sind hohe wie auch niedrige Restdehnungsbeträge festzustellen. 


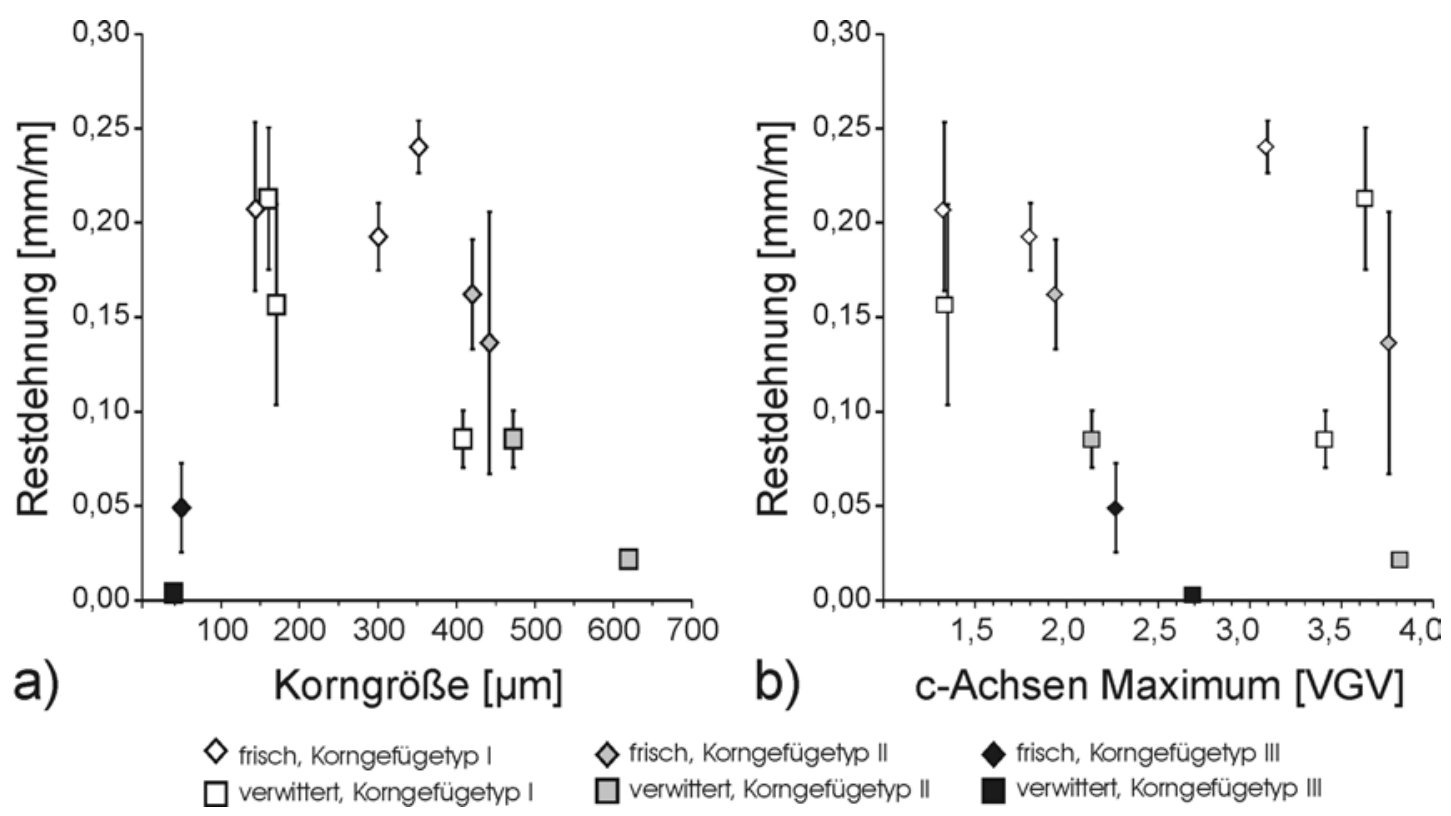

Abb. 5.10: Restdehnung verschiedener Marmore nach thermischer Beanspruchung auf $90{ }^{\circ} \mathrm{C}$ in Bezug zu a) der Korngröße und b) der Intensität der Textur (VGV $=$ Vielfaches der Gleichverteilung). Die Probenzustände (frisch, verwittert) und der jeweilige Korngefügetyp sind mittels verschiedener Symbole angegeben. Die vertikalen Linien repräsentieren die Anisotropie.

\subsubsection{Anstieg des Ausdehnungskoeffizienten}

Die ermittelten Längenänderungsdaten zeigen, dass bei zunehmender thermischer Beanspruchung, der Ausdehnungskoeffizient der Marmore ansteigt. Für unverwitterte Marmore ist vielfach ein sprunghafter Anstieg des Ausdehnungskoeffizienten zu ermitteln. Dieser ist auf das Wachstum von Mikrorissen zurückzuführen. Ein Anstieg des Ausdehnungskoeffizienten ist aber auch für vorgeschädigte Marmore festzustellen. Hierbei handelt es sich um einen Pufferungseffekt durch vorhandene Mikrorisse. Die Ursache hierfür ist eine verminderte Übertragung der Einkristalleigenschaften auf das Gesamtgestein aufgrund der Gefügeentfestigung.

In Dilatationsexperimenten kann beobachtet werden, dass der Anstieg der Dehnungskurven ab einer bestimmten Temperatur deutlich zunimmt (vgl. WIDHALM et al., 1996). Dieses Phänomen ist in Abb.5.11 für einen unverwitterten zyklisch beanspruchten Grosskunzendorfer Marmor dargestellt. Der Ausdehnungskoeffizient ist dabei im Temperaturbereich der Vorschädigung wesentlich geringer, als in Bereichen die nicht thermisch vorgeschädigt wurden. Besonders der dritte Heizzyklus auf $90^{\circ} \mathrm{C}$ zeigt, dass oberhalb der Temperatur des vorhergehenden Schädigungszyklus, der Kurvenverlauf und damit auch der Ausdehnungskoeffizient stark ansteigt. Das weist daraufhin, dass thermisch induzierte Rissbildung ab einer bestimmten kritischen Rissinitiierungstemperatur erfolgt („critical crack initiation temperature“: CCIT; vgl. RUEDRICH et al., 2001a), welche durch die entsprechende Vorschädigung oder auch durch die Sensibilität des jeweiligen Marmors gegenüber thermischer Beanspruchung bedingt sein sollte (vgl. auch WEISS et al., 2002). 


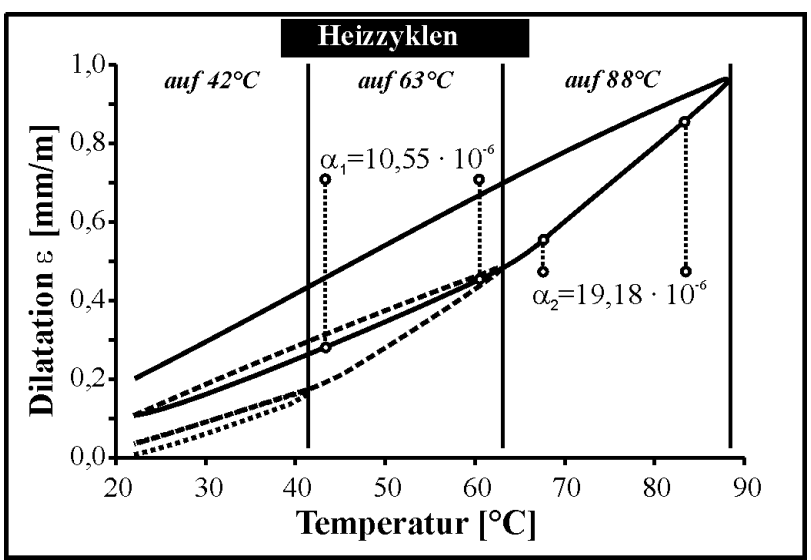

Abb. 5.11: Hysteresis eines zyklisch erhitzten Grosskunzendorfer Marmors: Oberhalb der vorhergehenden thermischen Schädigung ist der Ausdehnungskoeffizient wesentlich höher. Im dritten Heizzyklus bis $90{ }^{\circ} \mathrm{C}$ erreicht er einen doppelt so hohen Wert.

Generell kann auch für thermisch vorgeschädigte Marmore ein Anstieg des Ausdehnungskoeffizienten bei zunehmender Erwärmung beobachtet werden, der jedoch nicht auf eine Rissneubildung zurückgeführt werden kann. In Abb. 5.12a sind die experimentell ermittelten Ausdehnungskoeffizienten einer thermisch zuvor beanspruchten Probe aus Prieborner Marmor dargestellt. Zusätzlich sind in Abb. 5.12b nach den theoretischen Grundlagen von VoIGT (1928) berechnete Ausdehnungskoeffizienten in die X- und ZRichtung des Prieborner Marmors vergleichbar zu den experimentellen Daten aufgeführt. Die Berechnung erfolgte aus der Textur mittels der jeweils von KLEBER (1959) und von FEI (1995) angegebenen Ausdehnungskoeffizienten (s. Kap. 2.1). Im Vergleich der berechneten mit den experimentellen Daten ist $\mathrm{zu}$ erkennen, dass beide angenommenen Ausdehnungskoeffizienten hier keine Übereinstimmung liefern. Auffallend ist, das die experimentell ermittelten Ausdehnungskoeffizienten einen wesentlich steileren Anstieg aufzeigen, als selbst der nicht lineare stark ansteigende Koeffizient des Calcit-Einkristalls nach FEI (1995).
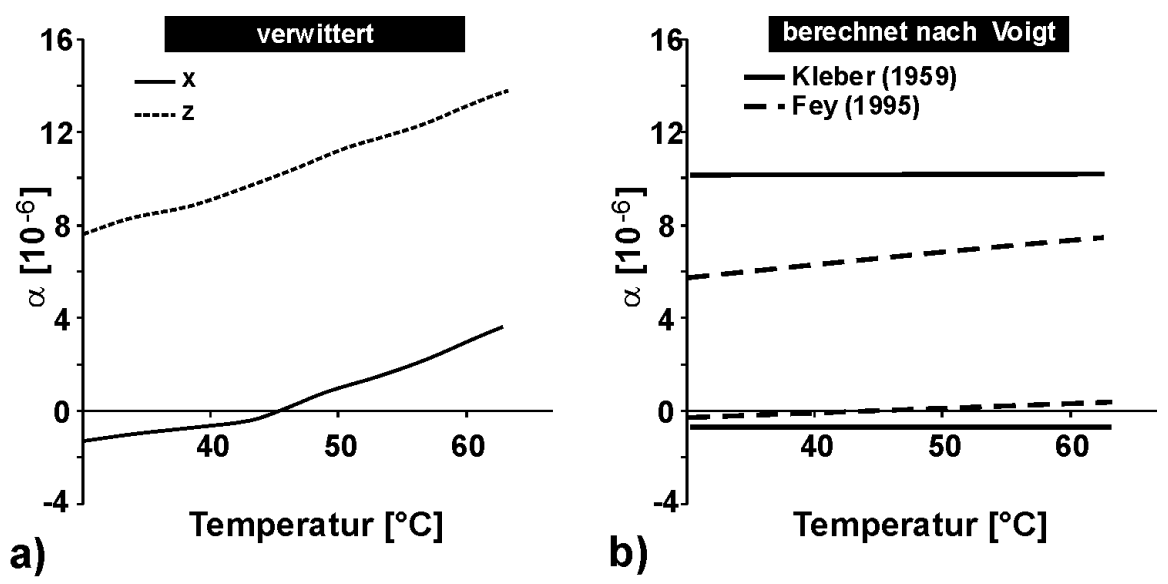

Abb. 5.12: Entwicklung der Ausdehnungskoeffizienten bei thermischer Beanspruchung für eine auf $65{ }^{\circ} \mathrm{C}$ vorgeschädigte Probe des verwitterten Prieborner Marmors: a) experimentell bei zunehmender Temperatur ermittelte und b) aus der Textur nach Voigt (1928) berechnete Ausdehnungskoeffizienten für die unterschiedlichen $\alpha$-Werte des Calcit-Einkristalls von KLEBER (1959) und von FeI (1995). 
Es ist anzunehmen, dass bei geringen Temperaturerhöhungen, ausgehend vom Ausgangzustand und beim Vorhandensein von Risssystemen, einige Kristalle die Möglichkeit haben sich in die vorhandenen Hohlräume hineinzudehnen bzw. im Fall parallel der a-Achsen die Rissporen zu weiten (s. Abb. 5.13; vgl. LeISS \& WeISS, 2000). Das bedeutet, falls senkrecht zur a-Achse eines Kristalls ein Riss existiert kann die Kontraktion nicht mehr auf das Gesamtgestein übertragen werden. Für höhere Temperaturbereiche ist davon auszugehen, dass die Dehnung zunehmend durch die starke Ausdehnung der c-Achsen getragen wird, da die Kontraktion der Kristalle parallel zu den a-Achsen nicht auf das Gestein übertragen wird. Dies erklärt auch den stark zunehmenden Ausdehnungskoeffizienten des Gesamtgesteins.

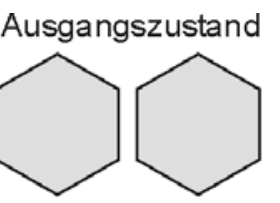

c-Achsen Kontakt
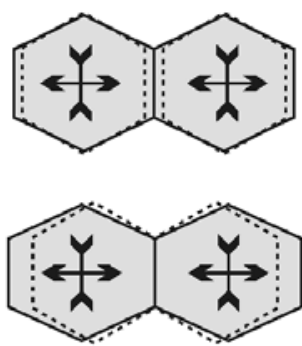

a) folgender Dehnung

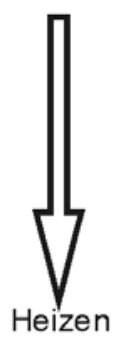

b)
a-Achsen Kontakt
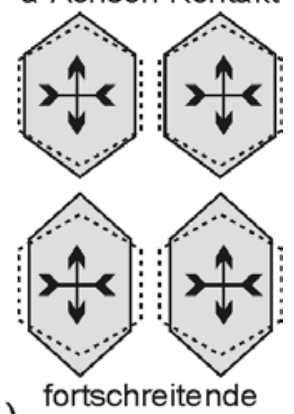

Rissweitung

Abb. 5.13 Schematische Darstellung von Dehnungsszenarien für Korn zu Kornbeziehungen bei thermischer Erwärmung wenn a) c-Achsen und b) a-Achsen zweier Körner aufeinander stehen und durch einen Mikroriss getrennt sind.

Abschließend kann festgehalten werden, dass Mikrorisse das Dehnungsverhalten der Marmore sehr stark beeinflussen. Dies kann zum einen durch eine Rissneubildung erfolgen, zum anderen vor allem durch einen Pufferungseffekt, der zu einer deutlich erhöhten Dehnung des Gesamtgesteins führt als aus den Einkristalldaten zu erwarten ist.

\section{2 Änderung verwitterungssensibler petrophysikalischer Parameter}

Die thermische Verwitterung in Verbindung mit Risswachstum führt zur Veränderung der petrophysikalischen Eigenschaften der Marmore. Um die Art und den Umfang dieser Änderungen des Materialverhaltens zu charakterisieren, erfolgte ein Vergleich von verwitterungssensiblen petrophysikalischen Parametern, welche vor und nach einer thermischen Beanspruchung ermittelt wurden. Dabei sind die, durch relativ geringe Temperaturbehandlungen von bis zu $90{ }^{\circ} \mathrm{C}$ hervorgerufenen Schädigungen, eindeutig mittels Ultraschalldiagnostik erfassbar. Die Veränderung des Porenraums, der kapillaren Wasseraufnahme und der Spaltzugfestigkeit liegen hier jedoch unterhalb der messtechnischen Auflösung. Daher wurden hier thermische Schädigungszyklen auf $200{ }^{\circ} \mathrm{C}$ gewählt. Im folgenden sind die Ergebnisse anhand von Beispielen der verschiedenen Korngefügetypen dargestellt. 


\section{- Zunahme der Porosität und Porengrößenverteilung}

Für alle untersuchten Marmore ist bei Wärmebehandlung von $200{ }^{\circ} \mathrm{C}$ eine deutliche Zunahme der Porosität und eine Verschiebung der Porengrößenklassen zu höheren Werten zu ermitteln. Exemplarisch sind die mittels Quecksilberporosimetrie ermittelten Daten für die verwitterten Proben des Prieborner (Korngefügetyp I) und des Sterzinger Marmors (Korngefügetyp II) in Abb. 5.14 dargestellt.

Die beiden Proben weisen im Ausgangszustand deutliche Unterschiede auf. Der Prieborner Marmor besitzt eine Porosität von $0,96 \%$, wobei seine häufigst besetzte Porenradienklasse zwischen $0,158 \mu \mathrm{m}$ und 0,2511 $\mu \mathrm{m}$ liegt. Obwohl die Probe des Sterzinger Marmors eine auffallend niedrigere Porosität von $0,50 \%$ aufweist, befindet sich die häufigst besetzte Porenradienklasse in einem vergleichbaren Abschnitt zwischen 0,2511 $\mu \mathrm{m}$ und 0,3981 $\mu \mathrm{m}$. Da die Porosität bei Rissporen durch das Verhältnis zwischen Rissdichte und Rissöffnung bestimmt wird, ist damit für den Sterzinger Marmor im Vergleich zum Prieborner Marmor eine deutlich geringere Häufigkeit der Risse festzustellen.
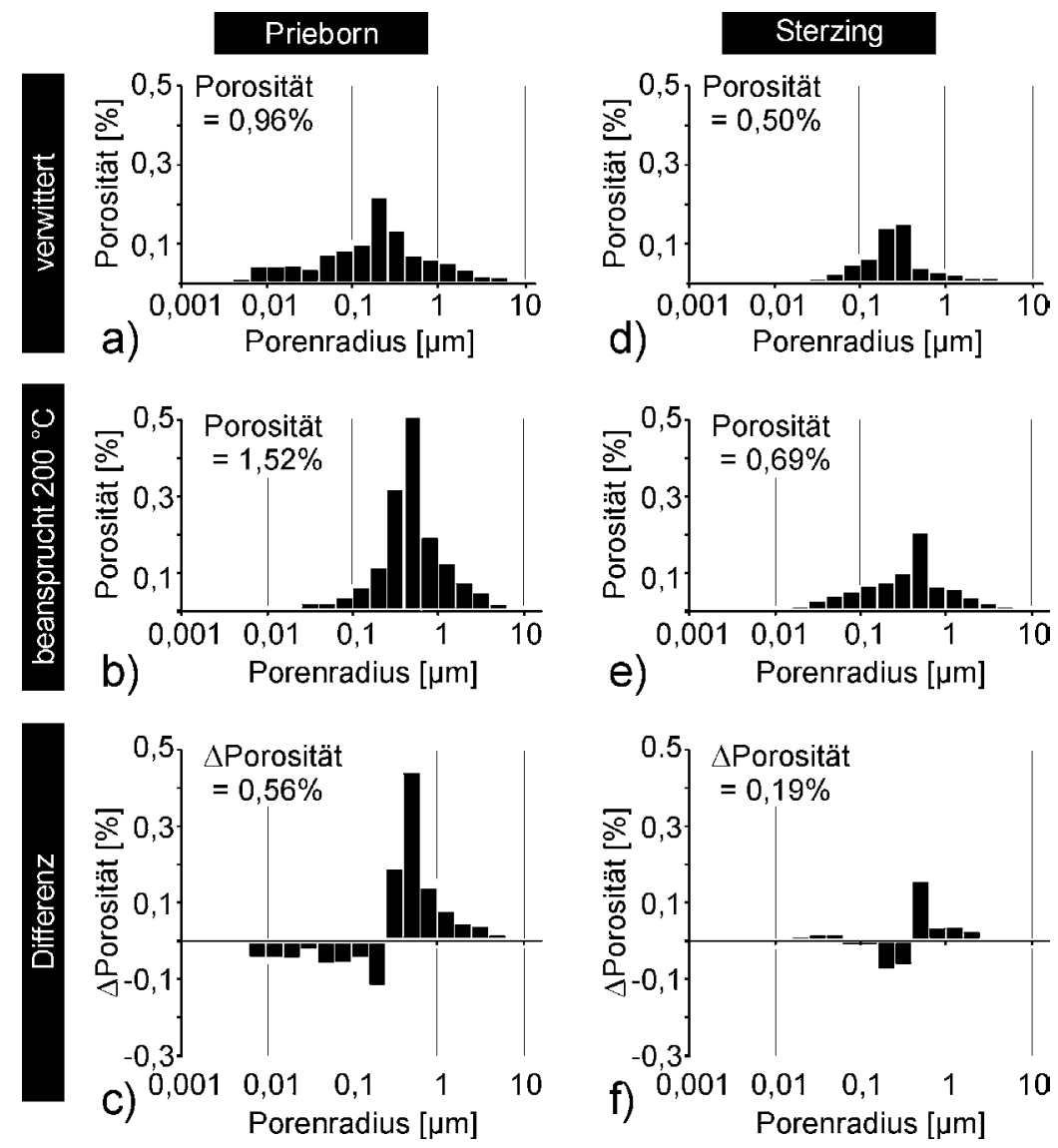

Abb. 5.14: Porosität und Porenradienklassenverteilung des Prieborner und des Sterzinger Marmors im a,d) verwitterten und im b,e) thermisch auf $200^{\circ} \mathrm{C}$ beanspruchten Zustand. In c,f) sind die $\Delta$-Porenradienverteilungen der beiden Probenzustände dargestellt.

Der Prieborner Marmor weist bei thermischer Beanspruchung auf $200{ }^{\circ} \mathrm{C}$ eine Erhöhung der Porosität von $0,96 \%$ auf 1,52 \% (Abb. 5.14a,b). Für den Sterzinger Marmor ist hingegen eine deutlich geringere Zunahme mit einem Anstieg der Porosität um 0,19\% auf 0,69\% festzustellen (Abb. 5.14d,c). Bezüglich der Veränderung der Porengrößenverteilung ist für 
beide Proben eine Erhöhung des Porenradienmaximum um ein bis zwei Stufen zu höheren Porenklassen $\mathrm{zu}$ ermitteln. Die Prieborner Probe weist zusätzlich eine ausgeprägte Verstärkung des Porenradienmaximums auf, d.h. bestimmte Porenradien werden besonders häufig herausgebildet.

Zusätzlich wurden $\Delta$-Porenklassendiagramme aus der Differenz der Verteilungen der verwitterten und der thermisch belasteten Probe errechnet (Abb. 5.14c,f). Sie zeigen, dass bei beiden Proben kleine Porengrößen abgebaut werden und größere deutlich zunehmen. Für den Sterzinger Marmor ist zusätzlich ein geringfügiger Zuwachs der Porenklassen zwischen $0,0158 \mu \mathrm{m}$ und $0,0631 \mu \mathrm{m} z u$ detektieren. Zwar ist die Zunahme in diesem unteren Bereich nur sehr gering und die entstandenen Radienklassen mit bis zu 0,0631 $\mu \mathrm{m}$ relativ groß, jedoch ist es ein Hinweis darauf, dass hier neue Risse gebildet wurden. Im Gegensatz dazu zeigt der Prieborner Marmor eine reine Abnahme der Porenklassen im unteren Radienspektrum, was eine vollständige Weitung vorhandener Risse aufzeigt.

\section{- Zunahme der kapillaren Wasseraufnahme}

Mit Zunahme der Porosität und der Weitung von Mikrorissen ist auch eine ausgeprägte Verstärkung der kapillaren Wasseraufnahme wärmebehandelter Marmore verbunden. Daneben kann sich auch die Differenz der aufgenommenen Wassermenge in die verschiedenen Gefügerichtungen des Gesteins verstärken. Als Beispiel für die Erhöhung der Wasseraufnahme bei thermischer Beanspruchung auf $200^{\circ} \mathrm{C}$ sollen die verwitterten Proben des Carrara (Korngefügetyp I) und des Grosskunzendorfer Marmors (Korngefügetyp II) dienen (Abb. 5.15). Die Messungen wurden an Würfelproben (65 mm Kantenlänge) in den drei orthogonalen Gefügerichtungen durchgeführt.

Für den Carrara Marmor ist eine Verdopplung der kapillar aufgenommen Wassermenge durch thermische Schädigung von ca. 0,1 Vol.-\% auf 0,2 Vol.-\% am Gesamtgestein zu ermitteln. Der damit verbundene Anteil der kapillar erreichbaren effektiven Porosität steigt dabei nur leicht von $19 \%$ auf $25 \%$. Weiterhin ist festzustellen, dass der Carrara Marmor sowohl im Ausgangs- als auch im thermisch beanspruchten Zustand, ein relativ isotropes Wasseraufnahmeverhalten aufweist. Dieses ist charakteristisch für die equigranular polygonalen Marmore des Korngefügetyp I und weist auf eine ausgeprägte 3-dimensionale Vernetzung der Korngrenzenrisse hin.

Der Grosskunzendorfer Marmor zeigt hingegen ein deutlich richtungsabhängiges Wasseraufnahmeverhalten. Die Anisotropie ist nach Mikrobereichsanalysen auf parallel verlaufende, sehr stark geweitete transgranulare Risse parallel zur XY-Ebene zurückzuführen. Bei thermischer Beanspruchung wird die Richtungsabhängigkeit noch verstärkt. Während die Wasseraufnahme parallel zur Z-Richtung annähernd gleich bleibt sind für die X- und auch für die Y-Richtung deutlich erhöhte Werte festzustellen. Parallel zur X-Richtung kann dabei die Würfelprobe eine erhöhte Menge Wasser von 0,34 Vol.-\% gegenüber 0,23 Vol.-\% im Ausgangszustand aufnehmen. Der Anteil der erreichbaren Porosität in diese Richtung verändert sich kaum und steigt von $31 \%$ auf $35 \%$. 

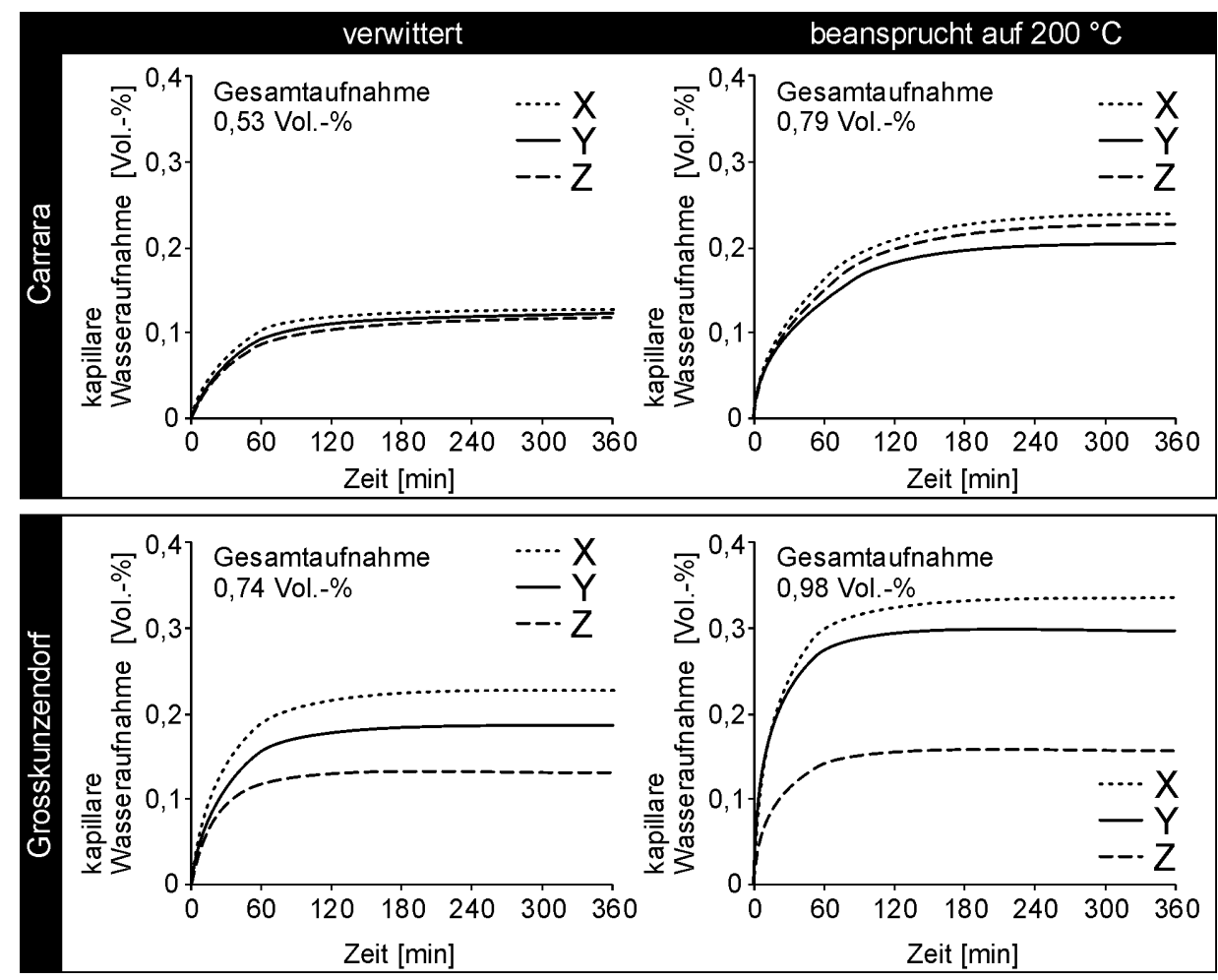

Abb. 5.15: Veränderung der kapillaren Wasseraufnahme nach thermischer Beanspruchung auf $200{ }^{\circ} \mathrm{C}$ für verwitterte Würfelproben (65 mm Kantenlänge) des Carrara und des Grosskunzendorfer Marmors.

\section{- Abnahme der Ultraschallgeschwindigkeiten}

Für alle Marmore ist eine Abnahme der Ultraschallgeschwindigkeiten nach thermischer Beanspruchung festzustellen. Dabei können Unterschiede in der Intensität und in der Richtungsabhängigkeit der Geschwindigkeitsherabsetzung ermittelt werden. Die Abnahme der P-Wellengeschwindigkeit infolge thermischer Beanspruchung auf $65{ }^{\circ} \mathrm{C}$ und $90{ }^{\circ} \mathrm{C}$ von Kugelproben ist exemplarisch in Abb. 5.16 für den Prieborner und in Abb. 5.17 für den Sterzinger Marmor dargestellt.

Für beide Proben ist eine deutliche Herabsetzung der Kompressionswellengeschwindigkeiten festzustellen. Im direkten Vergleich der beiden Marmore ist auffallend, dass Unterschiede der Herabsetzung für die jeweiligen Beanspruchungszyklen existieren. Für den Heizzyklus bis $65^{\circ} \mathrm{C}$ zeigt der Prieborner Marmor eine nur geringe mittlere $\mathrm{V}_{\mathrm{p}}$-Geschwindigkeitsreduktion von ca. $0,2 \mathrm{~km} / \mathrm{s}$ (vgl. Abb. 5.16d), wohingegen zum Heizzyklus auf $90{ }^{\circ} \mathrm{C}$ eine sehr deutliche Herabsetzung von ca. $0,7 \mathrm{~km} / \mathrm{s}$ zu beobachten ist. Im Gegensatz dazu zeigt der Sterzinger Marmor eine starke Herabsetzung von $0,5 \mathrm{~km} / \mathrm{s}$ beim Heizen auf $65^{\circ} \mathrm{C}$ und für den Heizzyklus bis auf $90{ }^{\circ} \mathrm{C}$ im Mittel nur eine geringe Veränderung der $\mathrm{V}_{\mathrm{p}}$-Geschwindigkeiten von ca. 0,2 km/s. Das gegensätzliche $\mathrm{V}_{\mathrm{p}}$-Verhalten der beiden Marmore ist hier auf eine kritische Rissinitiierungstemperatur zurückzuführen, die auf einer unterschiedlichen Vorschädigung der Proben oder einer gesteinsspezifischen Sensibilität gegenüber thermischer Beanspruchung beruht. 


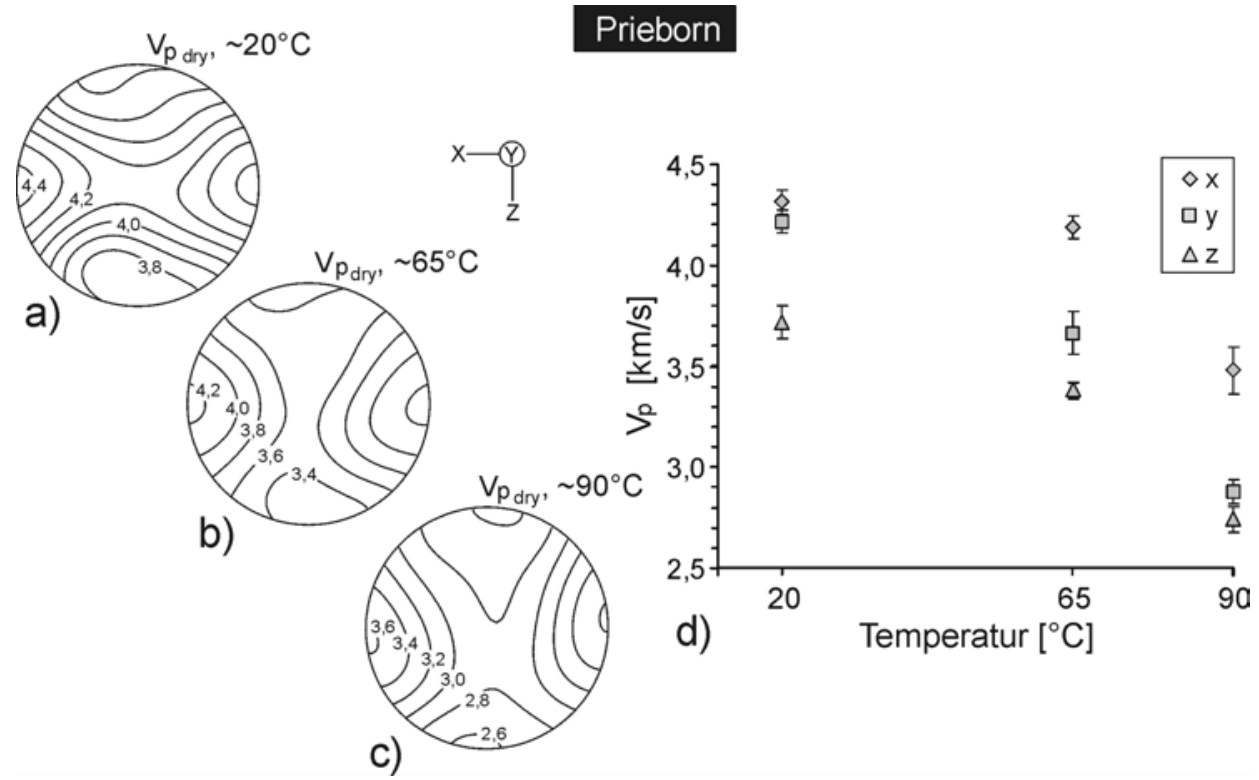

Abb. 5.16: Herabsetzung von $\mathrm{V}_{\mathrm{p}}$ (trockener Probenzustand) durch thermische Beanspruchung auf $65^{\circ} \mathrm{C}$ und $90^{\circ} \mathrm{C}$ einer Probe aus Prieborner Marmor: a-c) Ultraschallgeschwindigkeitsverteilung (Schmidt'sches Netz, untere Halbkugel) und d) entnommene Werte in den drei orthogonalen Gefügerichtungen.

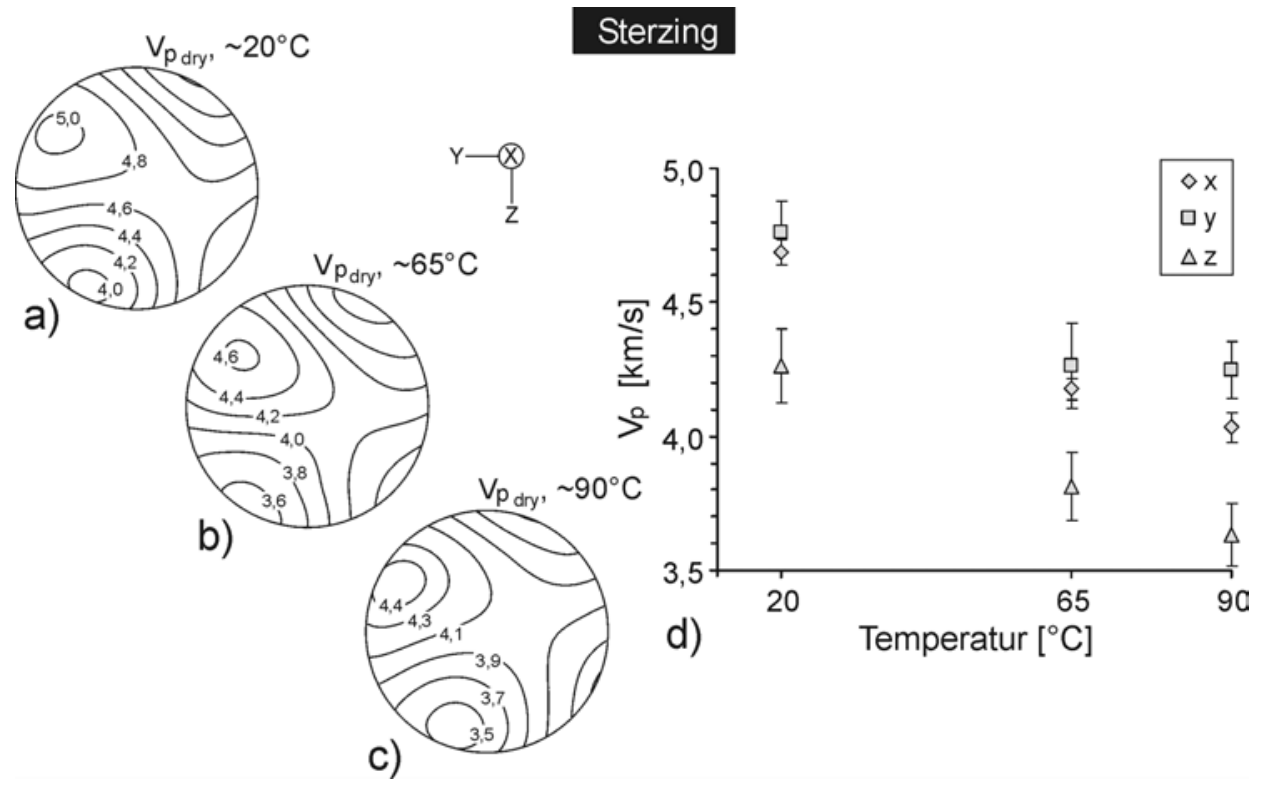

Abb. 5.17: Herabsetzung von $\mathrm{V}_{\mathrm{p}}$ (trockener Probenzustand) durch thermische Beanspruchung auf $65^{\circ} \mathrm{C}$ und $90^{\circ} \mathrm{C}$ einer Probe aus Sterzinger Marmor: a-c) Ultraschallgeschwindigkeitsverteilung (Schmidt'sches Netz, untere Halbkugel) und d) entnommene Werte in den drei orthogonalen Gefügerichtungen.

Die Richtungsabhängigkeit der P-Wellengeschwindigkeiten der Marmore wird bei beiden Heizzyklen nur im geringen Maße verstärkt. Der Sterzinger Marmor weist ein annähernd gleichbleibendes Muster der Geschwindigkeitsverteilungen auf (s. Abb. 5.17a-c). Für die Gesamtanisotropie der Prieborner Probe ist dies ebenfalls gegeben, jedoch kann eine signifikante Änderung parallel zur Y-Richtung beobachtet werden (s. Abb. 5.16d). In diese Richtung wird die Geschwindigkeit bei beiden Heizzyklen deutlich stärker herabgesetzt 
womit auch eine ausgeprägte Veränderung des $V_{\mathrm{p}}$-Verteilungsmusters einhergeht (s. Abb. 5.16a-c). Dieses Phänomen kann ebenfalls in den Restdehnungsintensitäten aus den Dilatationsmessungen ermittelt werden und ist auf ein bevorzugtes Risswachstum entlang einer Kornformregelung zurückzuführen (s. Tab. 5.2; vgl. Kap. 5.1.2).

Tab. 5.2: Geschwindigkeitsreduktionen und Restdehnungsbeträge (aus Messungen der thermischen Dilatation) des Prieborner und Sterzinger Marmors für Temperaturbeanspruchungszyklen auf $65^{\circ} \mathrm{C}$ und $90^{\circ} \mathrm{C}$.

\begin{tabular}{|c|c|c|c|c|c|c|c|c|}
\hline & Richtung & $\mathrm{V}_{\mathrm{p}} 20[\mathrm{~km} / \mathrm{s}]$ & $\mathrm{V}_{\mathrm{p}} 65[\mathrm{~km} / \mathrm{s}]$ & $\begin{array}{c}\Delta V_{p}= \\
V_{p} 20-V_{p} 65 \\
{[\mathrm{~km} / \mathrm{s}]} \\
\end{array}$ & $\begin{array}{l}\Delta \varepsilon 20-65 \\
{[\mathrm{~mm} / \mathrm{m}]}\end{array}$ & $\mathrm{V}_{\mathrm{p}} 90[\mathrm{~km} / \mathrm{s}]$ & $\begin{array}{c}\Delta V_{p}= \\
V_{p} 65-V_{p} 90 \\
{[\mathrm{~km} / \mathrm{s}]}\end{array}$ & $\begin{array}{l}\Delta \varepsilon 65-90 \\
{[\mathrm{~mm} / \mathrm{m}]}\end{array}$ \\
\hline \multirow{3}{*}{ 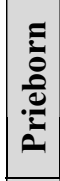 } & $\mathrm{x}$ & 4,31 & 4,19 & 0,12 & 0,062 & 3,48 & 0,71 & 0,161 \\
\hline & $\mathrm{y}$ & 4,21 & 3,67 & 0,55 & 0,117 & 2,88 & 0,79 & 0,247 \\
\hline & $\mathrm{z}$ & 3,72 & 3,38 & 0,34 & 0,113 & 2,74 & 0,64 & 0,206 \\
\hline \multirow{3}{*}{ 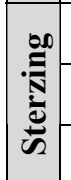 } & $\mathrm{x}$ & 4,69 & 4,18 & 0,51 & 0,028 & 4,03 & 0,14 & 0,083 \\
\hline & $\mathrm{y}$ & 4,76 & 4,26 & 0,50 & 0,037 & 4,25 & 0,01 & 0,099 \\
\hline & $\mathrm{z}$ & 4,26 & 3,81 & 0,45 & 0,060 & 3,63 & 0,18 & 0,121 \\
\hline
\end{tabular}

Eine Abnahme der Ultraschallgeschwindigkeiten kann in einem geringen Maße auch für die Herabkühlung von Marmoren festgestellt werden. Dies ist in Abb. 5.18 für eine Kugelprobe aus Lasa Marmor und einen Abkühlungszyklus von $20^{\circ} \mathrm{C}$ auf $-20^{\circ} \mathrm{C}$ dargestellt. Für die Xund Y-Richtung ist eine Herabsetzung der $\mathrm{V}_{\mathrm{p}}$-Geschwindigkeiten von ca. $0,3 \mathrm{~km} / \mathrm{s}$ festzustellen. Parallel zur Z-Richtung tritt lediglich eine Herabsetzung von ca. $0,15 \mathrm{~km} / \mathrm{s}$ auf.

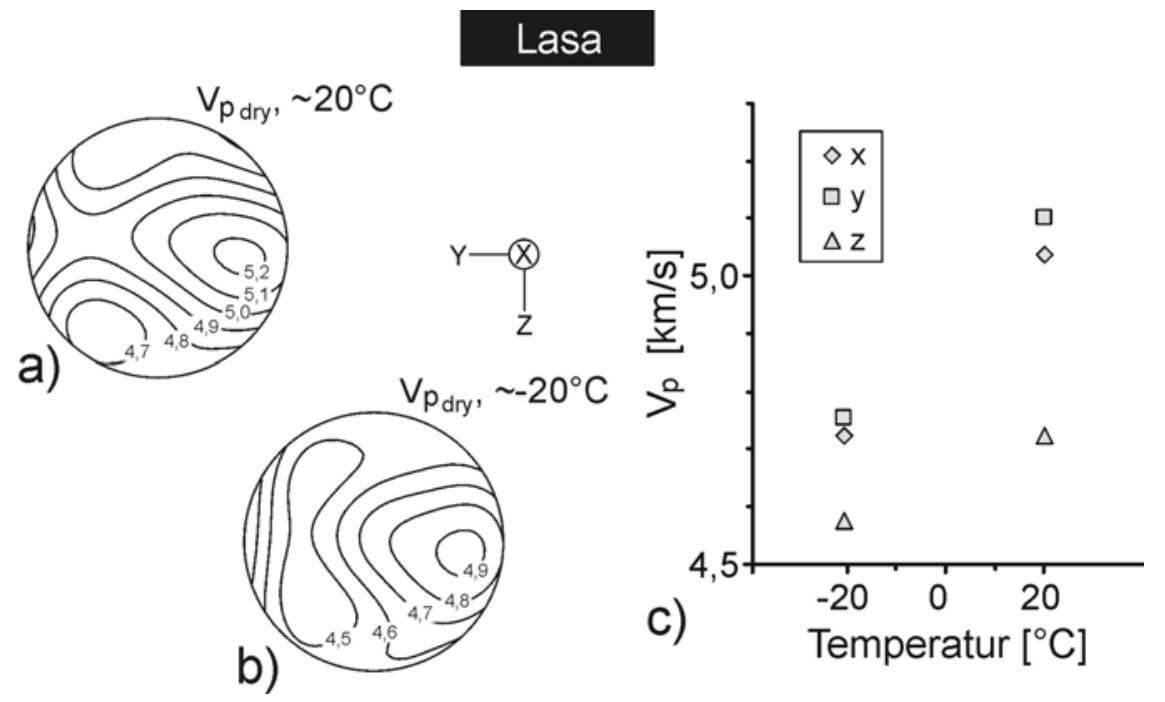

Abb. 5.18: Herabsetzung von $\mathrm{V}_{\mathrm{p}}$ (trockener Probenzustand) bei Abkühlung auf $-20{ }^{\circ} \mathrm{C}$ einer Probe aus Lasa Marmor: a,b) Ultraschallgeschwindigkeitsverteilung (Schmidt'sches Netz, untere Halbkugel) und c) entnommene Werte in den drei orthogonalen Gefügerichtungen.

In den Dilatationsmessungen sind hingegen auch für die untersuchte Probe aus Lasa Marmor keine Restdehnungen bei Abkühlung messbar. Damit kann festgestellt werden, dass die anisotropen Einkristalleigenschaften des Calcit sowohl beim Erwärmen als auch bei Abkühlung zu einer Schädigung eines Marmors führen können. Des weiteren repräsentieren 
Ultraschallmessungen auch im Vergleich $\mathrm{zu}$ den Längenänderungsmessungen eine hochauflösende Analytik mit der auch sehr geringe Probenveränderungen erfassbar sind.

\section{- Abnahme der Spaltzugfestigkeit}

Die thermische Beanspruchung von Marmoren führt zu einer Herabsetzung der Zugfestigkeiten. Um zu überprüfen in welcher Art und in welchem Umfang die Zugfestigkeiten der Marmore bei thermischer Beanspruchung herabgesetzt werden, wurden Probenscheiben ebenfalls bei $200{ }^{\circ} \mathrm{C}$ thermisch geschädigt. Die Untersuchungen erfolgten in den Richtungen maximaler und minimaler Zugfestigkeiten, an den zuvor richtungsabhängig charakterisierten Proben aus Carrara und Sterzinger Marmor (vgl. Kap. 4.3.4). Die Ergebnisse zeigen deutliche Unterschiede bezüglich der Sensibilität gegenüber thermischer Beanspruchung für die ausgewählten Marmortypen an. Dies betrifft sowohl die Intensität der Festigkeitsherabsetzung als auch ihre Richtungsabhängigkeit.

Für die thermische Beanspruchung der Probe aus Carrara Marmor ist lediglich eine Abnahme von $0,20 \mathrm{MPa}$ in der maximalen und $0,28 \mathrm{MPa}$ in der minimalen Spaltzugfestigkeitsrichtung festzustellen (Abb. 5.19a). Die ermittelten Zugfestigkeitsabnahmen liegen damit unterhalb der messtechnischen Auflösung dieser Methode. Der Sterzinger Marmor zeigt im Gegensatz dazu eine höhere Abnahme der Spaltzugfestigkeiten (Abb. 5.19c). Für die thermische Beanspruchung auf $200^{\circ} \mathrm{C}$ ist eine annähernde Halbierung der Spaltzugfestigkeit in maximaler Richtung um 3,54 MPa zu ermitteln. Für die Richtung minimaler Zugfestigkeit ist
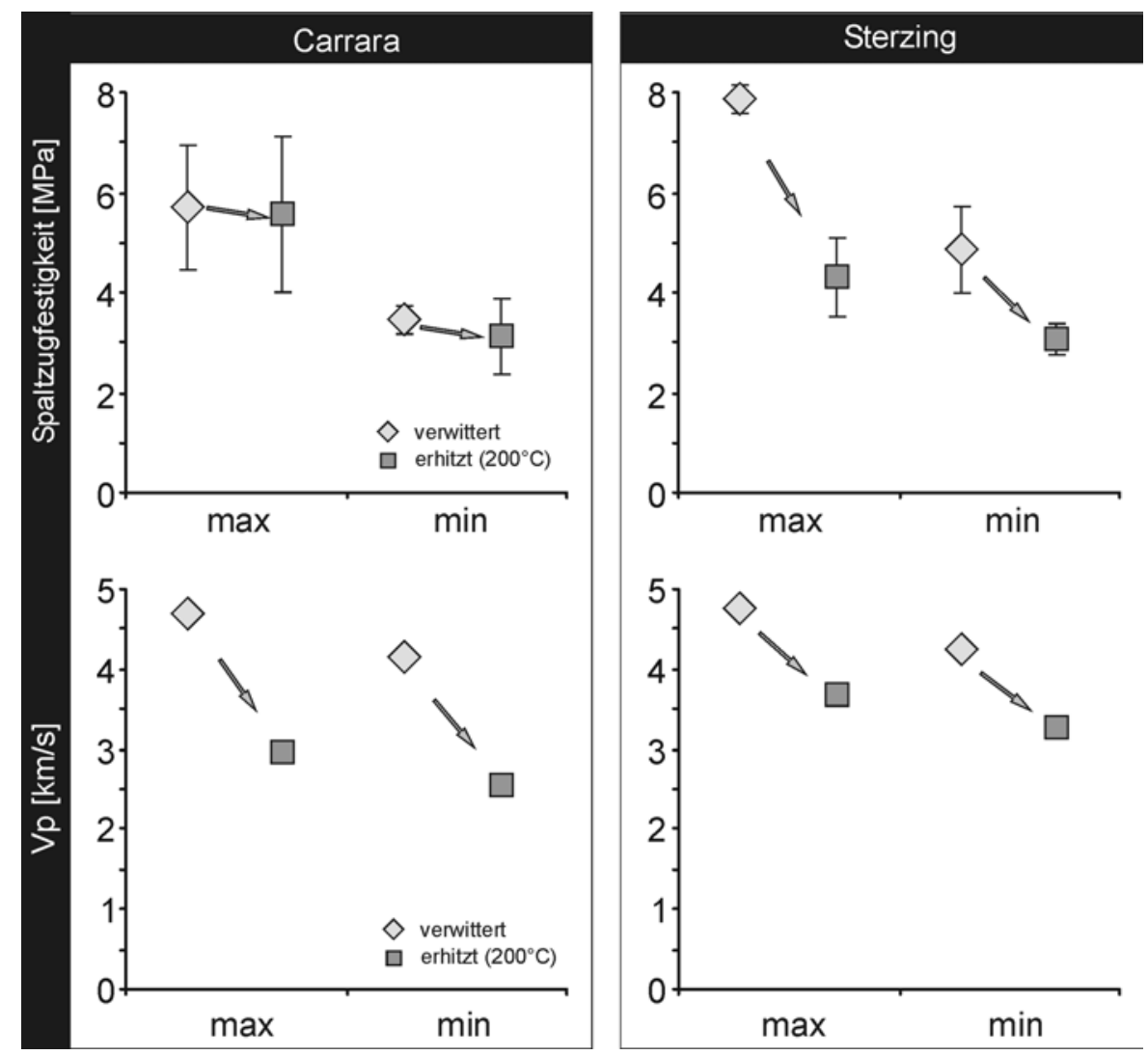

Abb. 5.19: Reduktion der Spaltzugfestigkeit und der Kompressionswellengeschwindigkeiten durch thermische Beanspruchung auf $200^{\circ} \mathrm{C}$ für den verwitterten Sterzinger und Carrara Marmor. 
eine etwas geringere Reduktion von 1,75 MPa festzustellen. Da die Richtung maximaler Spaltzugfestigkeit eine ausgeprägtere Abnahme aufweist, ist somit auch eine Herabsetzung der Anisotropie von $\mathrm{A}=38 \%$ auf rund $\mathrm{A}=9 \%$ verbunden.

Zum Vergleich sind in Abb. 5.19b,d die Abnahme der Ultraschallgeschwindigkeiten der entsprechenden Marmorproben bei vergleichbarer thermischer Beanspruchung dargestellt. Die Daten zeigen ein auffällig geändertes Bild verglichen mit den Festigkeitsdaten. Während für den Carrara Marmor eine kaum auflösbare Herabsetzung der Festigkeiten festgestellt werden kann, ist die Reduktion von $\mathrm{V}_{\mathrm{p}}$ sehr stark ausgeprägt. Hingegen zeigt der Sterzinger Marmor eine geringere Abnahme der Ultraschallgeschwindigkeiten aber eine starke Abnahme der Spaltzugfestigkeiten.

Die Ergebnisse zeigen, das die Abnahme der Zugfestigkeit nicht zwangsläufig mit dem Risswachstum korreliert. Dabei ist für eine Rissweitung davon auszugehen, dass keine erhebliche Gefügeschwächung und damit Abnahme der Kohäsion verbunden ist, da das Gefüge schon weitgehend entfestigt ist. Eine starke Schwächung ist nur für eine Rissneubildung anzunehmen. 


\section{Modellhafte Konservierung verwitterter Marmore}

Seit langem wird versucht, dem voranschreitenden Verfall von Gesteinen entgegenzuwirken und ihre Widerstandsfähigkeit gegenüber der Verwitterung zu erhöhen. Je nach Schadensphänomenen und Gesteinstyp stellen sich dabei entsprechende Anforderungen an das einzusetzende Verfahren (CLIFTON, 1980). Es wird unterschieden zwischen Maßnahmen die einer Verbesserung der Gefügekohäsion dienen, dies sind Steinfestigungen, und Maßnahmen welche gezielt präventiv Verwitterungsprozesse verlangsamen sollen, wie z.B. Hydrophobierungen. Eine Übersicht über historische Steinkonservierungsverfahren gibt WEBER (1985).

Für Marmore ist das typische Schadensszenario das fortschreitende Mikrorisswachstum mit einhergehender gesteinsdurchdringender Gefügeentfestigung, welches im Endstadium zu einem sandigen Materialverlust einzelner Partikel führt. Aus diesen Gründen werden für verwitterte Marmore vor allem Steinfestigungsmaßnahmen eingesetzt, bei der eine Substanz in den Porenraum eingebracht wird, welche die Fähigkeit besitzt eine Bindung der entfestigten Komponenten wiederherzustellen. Aus heutiger Sicht können dabei drei grundsätzliche Wirkprinzipien unterschieden werden (vgl. AURAS et al., 1997):

1.) Abscheidung eines Kunstharzfilms der die Körner überzieht und klebende Wirkung besitzt

2.) Abscheidung eines mineralischen Bindemittels, also einer porenraumfüllenden Substanz, welche zur Erhöhung der Festigkeiten beiträgt

3.) Volltränkung mit weitgehender Schließung der Porenräume, und Verklebung der einzelnen Komponenten, mit weitgehender Wiederherstellung der ursprünglichen Festigkeit

Das erste Wirkprinzip beruht auf der Einbringung eines elastischen Kunststoffes in den Porenraum, der die mineralischen Komponenten überzieht und in Form einer lokalen Verklebung zu einer Festigkeitserhöhung führt. Hierzu finden heute vor allem Reaktionsharze wie Polyurethan-, Epoxid- und Acrylharze Verwendung. Durch die Verringerung der Porosität soll auch die Aufnahme von flüssigem Wasser vermindert werden. Um das Trocknungsverhalten nicht negativ zu beeinflussen muss jedoch ein Wasserdampftransport erhalten bleiben (HoNSINGER \& SASSE, 1988). Die oben genannten Kunststoffe können eine große Spannbreite an Eigenschaften aufweisen, die von der chemischen Zusammensetzung der Monomere, den chemischen Bindungsarten, der Form der Verknüpfung und der Größe der Makromoleküle abhängen. Z.T. können in bestimmten Grenzen Festigkeit, Verformbarkeit, thermische Beständigkeit und die Stabilität gegenüber UV sowie chemischen Angriffen eingestellt werden. Jedoch ergeben sich für jedes Kunstharz eigene Problematiken, wie 
generelle hydrophobierende Eigenschaften von Polyurethan oder bei normaler Reaktionsgeschwindigkeit Vergilbung von Epoxidharz.

Im Gegensatz dazu steht das zweite Wirkprinzip, die Abscheidung von mineralischem Bindemittel, welches im Porenraum keinen Film erzeugt, der die Körner überzieht, sondern vielmehr lokal zur Ausfällung einer stützenden Substanz führt. Dies sind in erster Linie Kieselgel-abscheidende Mittel, wobei heute fast nur noch der Kieselsäureesther (KSE) von Bedeutung ist. Der KSE eignet sich nur für spezifische Schäden als Schutzmittel. Dies sind Oberflächenentfestigungen in Form des feinkörnigen Absandens oder bei tiefgründigen Entfestigungsarten. Für die Festigung von Abplatzungen, Schalen oder weit geöffneten Rissen ist er jedoch aufgrund fehlender Klebkraft ungeeignet.

Beide genannten Wirkprinzipien führen zu einer Veränderung der Porenraumeigenschaften. So werden die häufig durch die Verwitterung vergrößerten Porositäten verringert aber auch die Porengrößenverteilung modifiziert. Da die Wassertransportmechanismen im Naturstein insbesondere von diesen Parametern im wesentlichen gesteuert werden, können erhebliche Veränderungen im Wasserhaushalt der Gesteine auftreten.

Das dritte Wirkprinzip, die Volltränkung, verfolgt hingegen ein anderes Ziel. Dabei soll eine vollständige Imprägnierung den entfestigten Naturstein verkleben, die Festigkeit erhöhen und den Eintritt von Wasser verhindern. Letzteres ist nur bei Tränkung des gesamten Bauteils sichergestellt. Daher wird dieses Verfahren zur Zeit nicht vor Ort angewandt, sondern die Objekte müssen abgebaut und zur Tränkungsanlage transportiert werden. Daraus resultiert eine eingeschränkte Einsetzbarkeit in der Denkmalpflege, da viele Objekte nicht oder nur schwer aus Bauwerken entfernt werden können.

\subsection{Konservierungsmittel und Applikation}

Basierend auf den angeführten Wirkprinzipien wurden für die vorliegende Arbeit drei Konservierungsverfahren ausgewählt und eingesetzt. Ein Polymethylmetacrylat auf Lösungsmittelbasis $\left(\mathrm{PMMA}_{\text {sol }}\right.$ ) sowie ein Polykieselsäureester (PKSE) ebenfalls in gelöster Form. Als drittes Verfahren wurde die Acrylharzvolltränkung (PMMA poly) angewandt. Konserviert wurden die natürlich verwitterten Proben der ausgewählten Marmore, wobei zusätzlich eine thermische Vorkonditionierung erfolgte.

\section{- Polymethylmetacrylat auf Lösungsmittelbasis (PMMA sol)}

Das verwendete $\mathrm{PMMA}_{\text {sol }}$ ist ein speziell modifiziertes Polymethylmetacrylat mit einem geringen Anteil eines Polymethyl-butyl-copolymers und einigen sauren Gruppen zur besseren Haftung an den Calcit-Oberflächen (vgl. KoBLISCHEK, 1990). Das Acrylharz hat eine Molekülgröße von $24 \mathrm{~nm}$ und eine Dichte von $0,95 \mathrm{~g} / \mathrm{cm}^{3}$. Die Glasübergangstemperatur $\left(\mathrm{T}_{\mathrm{g}}\right)$ liegt bei ca. $60^{\circ} \mathrm{C}$. Für die Konservierung wurde als Lösungsmittel Xylol verwendet, wobei der Festigergehalt bei ca. 40 \% liegt. Der Festiger bekommt Effektivität nach der Verdunstung des Lösungsmittels und formt Filme unterschiedlicher Stärke. 


\section{- Polykieselsäureester (PKSE)}

Der PKSE ist ein Ethylsilikat welches aus 3 bis 5 Kieselsäureester-Molekülen besteht. Das Oligomer hat eine Molekülgröße zwischen 1,2 - $6 \mathrm{~nm}$ wobei die Fraktion um $3 \mathrm{~nm}$ dominiert (KoBlischeK, 1990). Der PKSE ist gelöst in Ethanol mit einem aktiven Wirkstoffgehalt von $40 \%$.

Kieselsäureester (KSE, Summenformel $\mathrm{Si}(\mathrm{OR})_{4}$ ) sind Flüssigkeiten die durch Umsetzung von Kieselsäure mit Alkohol hergestellt werden. Dabei ist - $\mathrm{R}$ die Alkylgruppe, die bei dem verwendeten PKSE durch den Ethylrest $-\mathrm{C}_{2} \mathrm{H}_{5}$ gebildet wird. Während der Festigung laufen verschiedene Reaktionen ab, wobei letztendlich der KSE aus der Reaktion mit Wasser und unter Abspaltung von Alkohol ein amorphes Kieselgel bildet. Die Hydrolyse durch Reaktion mit Wasser erfolgt nach folgender chemischer Reaktion:

$$
\mathrm{Si}(\mathrm{OR})_{4}+\mathrm{nH}_{2} \mathrm{O} \rightarrow \mathrm{SiO}_{2} \cdot \mathrm{aq}+\text { Alkohol }
$$

Bei vorkondensierten Produkten, wie dem verwendeten PKSE, ist die Hydrolyse teilweise vorweggenommen. Der neugebildete Alkohol und etwaige Lösungsmittel verdampfen und zurück bleibt das Kieselgel. Das in das Gel eingelagerte Wasser verdunstet im Laufe der Zeit, wodurch das Gel schrumpft. Die Folge sind die charakteristischen Schrumpfrisse im ansonsten glasähnlichen $\mathrm{SiO}_{2}-\mathrm{Gel}$ (vgl. auch KoBLISCHEK, 1997).

Die PMMA sol- $^{-}$und die PKSE-Festiger werden normalerweise für Konservierungen am Bauwerk verwendet. Sie werden gewöhnlich auf die Gesteinsoberfläche aufgetragen und durch Kapillarkräfte in das Innere des Gesteins aufgenommen. Um eine ausreichende Eindringtiefe $\mathrm{zu}$ erzielen, werden beide Verfahren vor Ort mit einem höheren Lösungsmittelanteil verwendet.

Um eine weitgehende und homogene Verteilung des Imprägnierungsmittels zu erreichen, wurden in dieser Arbeit die Konservierungsmittel unter Vakuum von 0.07 bar eingebracht und anschließend einem Überdruck von 6 bar ausgesetzt. Um die Vernetzung der Rissporen zu verbessern, wurden die Proben durch Heizen auf $200{ }^{\circ} \mathrm{C}$ vorkonditioniert. Diese Vorgehensweise war für die besonders stark verwitterte Probe des Carrara Marmors nicht notwendig.

\section{- Acrylharzvolltränkung (PMMA poly)}

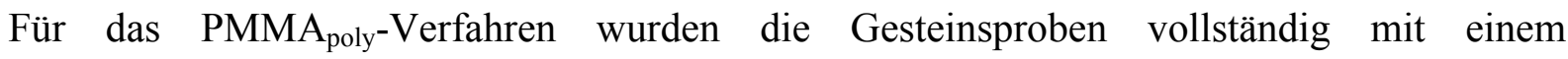
Methylmetacrylat imprägniert und im Gestein polymerisiert. Das so generierte $\mathrm{PMMA}_{\text {poly }}$ ist ein Plexiglas mit einem $\mathrm{T}_{\mathrm{g}}$ von ca. $85^{\circ} \mathrm{C}$ und einem thermischen Ausdehnungskoeffizienten $(\alpha)$ of $70 \cdot 10^{-6} \mathrm{~K}^{-1}$. Die Tränklösung beinhaltet ferner ein Acrylsilan und eine bestimmte Menge Acrylsäure (LORENZ \& IBACH, 1999). Die Volumenreduktion während der Polymerisation beträgt ungefähr 20 Vol.-\%.

Der Verfahrensablauf der Acrylharzvolltränkung beginnt mit der Konditionierung des Probenmaterials durch Trocknung bei ca. $70{ }^{\circ} \mathrm{C}$. Die Objekte werden in einen Tränkbehälter eingelegt und in einen Vakuum-Druckkessel eingebracht. Nach einer Vakuum-Phase von mehreren Stunden wird der Tränkbehälter bis zur vollständigen Bedeckung der zu tränkenden Objekte mit monomerem Methylmetacrylat geflutet. Nach mehreren Vakuum-/Druckphasen 
(0.75/25 bar) wird die Polymerisation durch Erhöhung der Temperatur auf ca. $80{ }^{\circ} \mathrm{C}$ eingeleitet. Eine detaillierte Beschreibung des Volltränkungsverfahrens befindet sich in WIHR (1995) und AURAS et al. (1997) sowie ergänzende Details in LORENZ \& IBACH (1999).

\subsection{Vorkommen und Verteilung der Konservierungsmittel im Porenraum}

Das Vorkommen und die Verteilung des jeweiligen Konservierungsmittels im Porenraum der Marmore beinhaltet wichtige Informationen über die Füllung der Poren sowie die Haftung (Adhäsion) des Imprägnierungsmittels an den Calcitoberflächen. Darüber hinaus können Einschätzungen über die Modifikation des Porenraums wie sekundäre Porosität und Vernetzung der Poren erfolgen. Für die verschiedenen Konservierungsmittel ist dabei ein individuelles Vorkommen im Porenraum der Marmore zu ermitteln (s. Abb. 6.1).

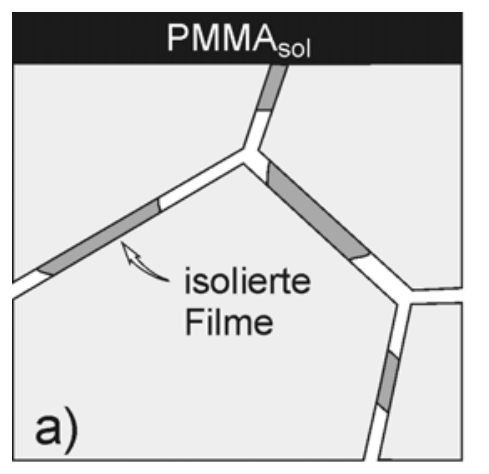

Porenraum

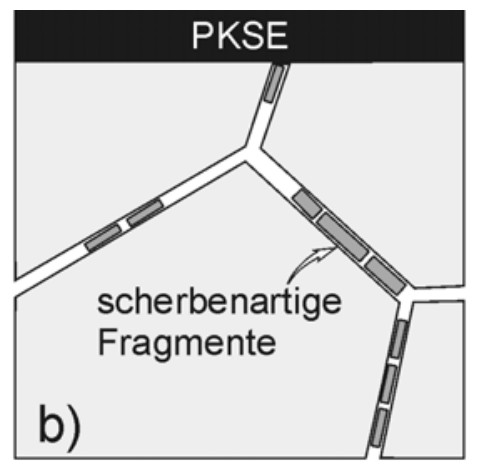

Kristalle

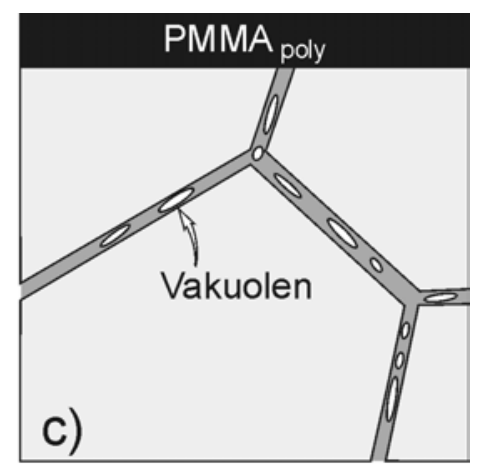

Festiger

Abb. 6.1: Schematische Darstellung des Vorkommens der verwendeten Konservierungsmittel im Porenraum der Marmore.

Das Vorkommen des $\underline{\text { PMMA }}_{\text {sol }}$ im Porenraum der Marmore ist nach REM-Untersuchungen (Fraktographie) zumeist durch lokale, isolierte Kunststofffilme auf Kristalloberflächen gekennzeichnet (s. Abb. 6.1a). Diese variieren in Form und Größe. Dabei ist die Ausbildung und die Häufigkeit der auftretenden Filme abhängig von den Porengrößen und damit vom Gesteinszustand. Im Porenraum des stark verwitterten Carrara Marmors mit relativ großen Porendurchmessern erscheinen die Filme als ausgedehnte Überzüge auf Korngrenzen (s. Abb. 6.2).

Diese Beobachtung gilt ebenfalls für den Prieborner und den Grosskunzendorfer Marmor. Hingegen weisen die Marmortypen mit reduzierten Porenräumen, wie der Laaser und der Sterzinger, nur sehr vereinzelt und nur sehr kleine, fleckenartig auftretende Filme auf. Häufig zeigen die, durch das Aufbrechen der Probe sichtbar gemachten Oberflächen, Kornabdrücke, die auf eine lokale Verklebung der Körner hinweisen. Weiterhin sind die PMMA sol-Filme gekennzeichnet durch oft löchrig erscheinende Partien und unregelmäßige Formen. Die Ränder des Festigers sind dabei gerundet und daher nicht als abgerissene Relikte der Probenpräparation anzusehen (s. Abb. 6.2). Vielmehr können sie als Schrumpfungserscheinungen, die sich während der Verdunstung des Lösungsmittels entwickelt haben, interpretiert werden. 

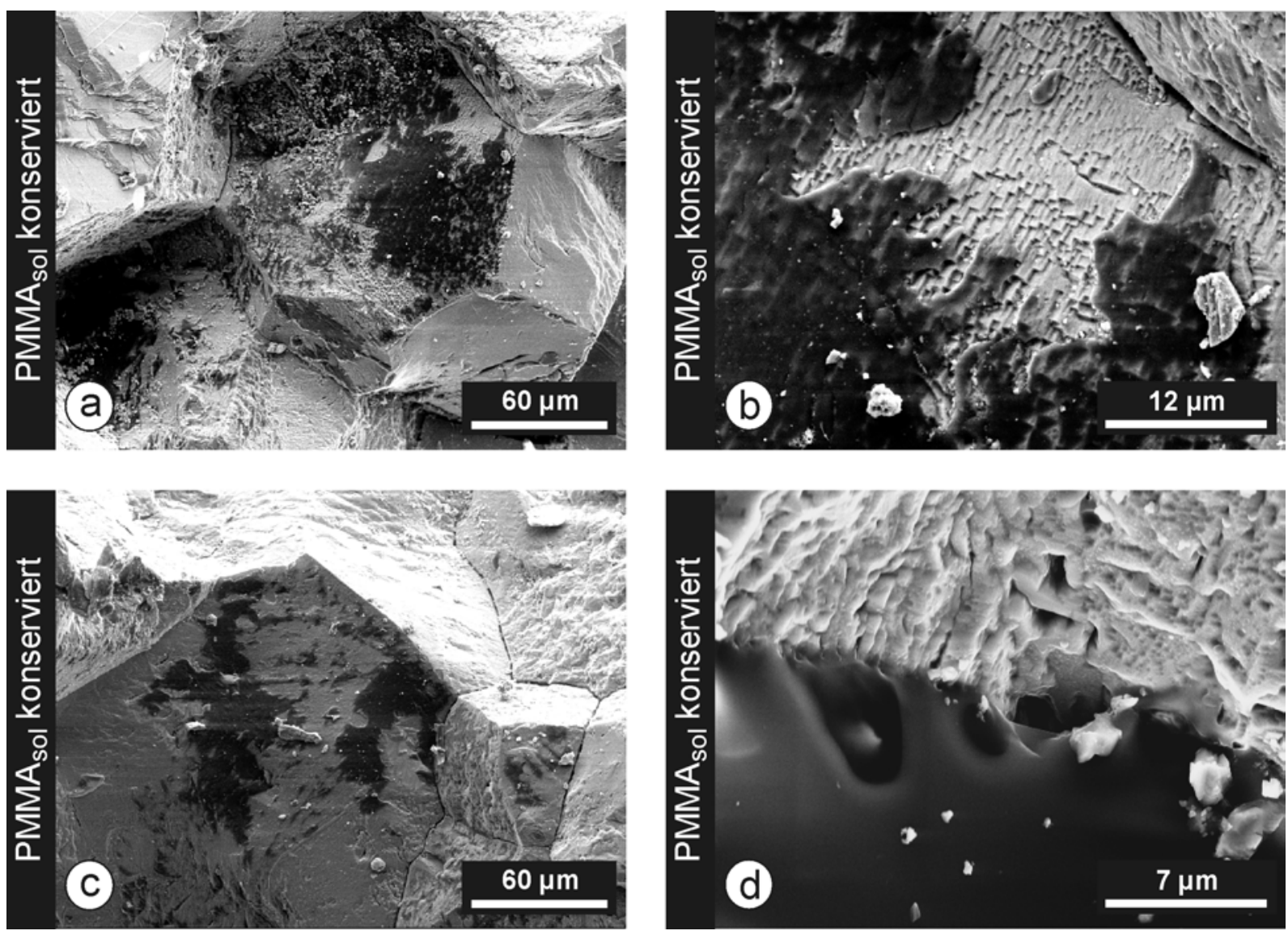

Abb. 6.2: REM-Aufnahmen von PMMA $\mathrm{A}_{\text {sol }}$ konservierten Marmorproben (Fraktographie): a-c) lokales Vorkommen der Kunststofffilme auf Korngrenzen des stark verwitterten Carrara Marmors und d) Anhaftung des Acrylharzes auf einer Kristalloberfläche.

Der $\underline{\text { PKSE }}$ erscheint im Porenraum der Marmore als unregelmäßige, scherbenartige Fragmente, die von Rissen getrennt sind (s. Abb. 6.1b). Zumeist weisen die Scherben plattenartige, polygonale Formen auf, womit die Gesamtstruktur häufig pflasterartig erscheint (s. Abb. 6.3a). Diese Erscheinungsform des PKSE kann auf die Schrumpfung während der Gelbildung zurückgeführt werden (vgl. KoBLISCHEK, 1990). Die Größe der einzelnen Fragmente variiert um $5 \mu \mathrm{m}$ bis $30 \mu \mathrm{m}$. Die Risse zwischen den einzelnen Fragmenten besitzen zumeist um $1 \mu \mathrm{m}$ Durchmesser. Die Tatsache, dass auf den Flächen des PKSE-Gels kein negativ Abdruck der Kornoberflächen zu beobachten ist, deutet auf nur geringe oder fehlende Kohäsion zwischen Gel und Calcit Oberfläche hin (s. Abb. 6.3).

Das $\underline{\text { PMMA }}_{\text {poly }}$ des Volltränkungverfahrens erscheint in REM-Aufnahmen als dunkler, durchhaltender Überzug auf den Kristalloberflächen (s. Abb. 6.4a,b). Die Negativabdrücke von Spaltflächen und Korngrenzenmorphologien auf abgerissenen Kunststoffschichten sind ein Indiz dafür, dass der Kunststoff die Risse weitgehend ausgefüllt hat (s. Abb. 6.4a). Ebenfalls ist damit von einer guten Haftung zwischen PMMA $_{\text {poly }}$ und der Kristalloberflächen auszugehen. In Schnitten senkrecht zu den gefüllten Rissen sind kleine, zumeist ovale Löcher mit einer Größe bis zu $1 \mu \mathrm{m}$ zu beobachten. Sie erscheinen in den zentralen Bereichen des Kunststoffs (s. Abb. 6.4c,d). Die Löcher sind als Vakuolen zu interpretieren, deren Entstehung auf die Volumenreduktion des $\mathrm{PMMA}_{\text {poly }}$ während der Polymerisation zurückzuführen ist (vgl. LORENZ \& IBACH, 1999). 

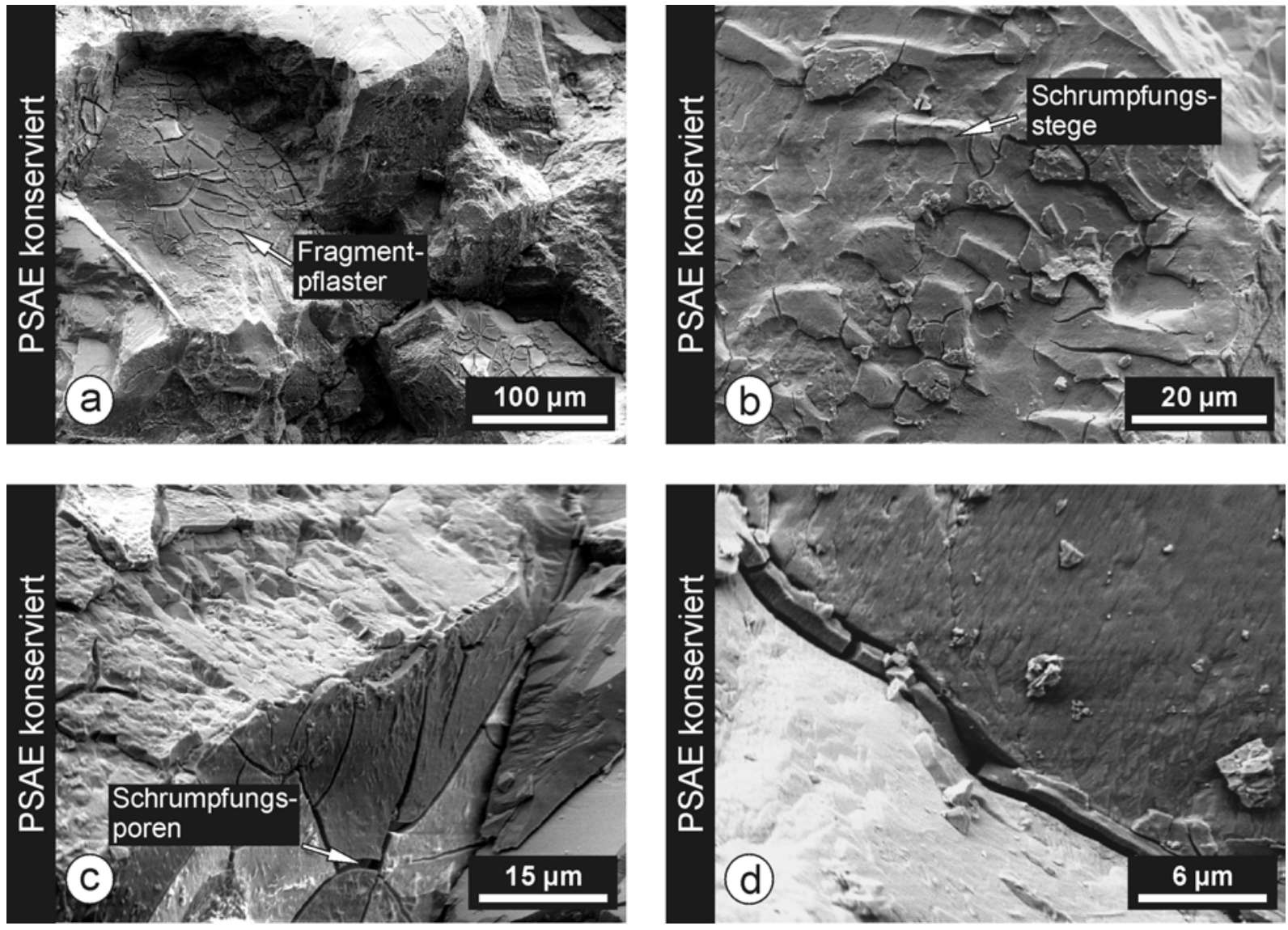

Abb. 6.3: REM-Aufnahmen von PKSE konservierten Marmorproben (Fraktographie): a) pflasterartiges Vorkommen von Gelfragmenten auf einer Korngrenzenfläche, b) durch Schrumpfung erzeugte Stege von Kieselgel, c) Gelfragmente welche durch Schrumpfungsrisse getrennt sind und d) Ausfüllung eines Korngrenzenrisses mit Gelfragmenten.

\subsection{Konservierungsbedingte Änderungen petrophysikalischer Parameter}

Die Konservierung von entfestigten Marmoren durch Steinfestiger führt zu einer mehr oder weniger deutlichen Änderung der petrophysikalischen Eigenschaften des Gesteins. Dazu gehören die Porenraumeigenschaften und die damit verbundenen Wassertransportmechanismen. Durch den Verbund der Körner ändern sich ebenso die elastischen Eigenschaften, was zu einem Anstieg der Ultraschallgeschwindigkeiten führt. Resultierend erhöht sich auch die Spaltzugfestigkeit.

\section{- Abnahme der Porosität und Veränderung der Porenradienverteilung}

Durch das Einbringen eines Festigers in den Porenraum eines Marmors nimmt zwangsläufig die Porosität $a b$ und das Porenradienspektrum verändert sich. Das Maß der Abnahme der effektiven Porosität durch eine Konservierung gibt dabei Informationen über die Art und Weise in welcher die Reduktion erfolgt. Dabei weisen z.B. sehr starke Abnahmen auf eine Verschließung der Zugänglichkeit von Poren hin, wie durch Verklebung von Porenkanälen mit der Entstehung einer Totporosität. Darüber hinaus gibt der Vergleich der Porengrößenverteilung vor und nach einer Konservierung Auskunft über die bevorzugte 
Besetzung bestimmter Porengrößen durch das jeweilige Imprägnierungsmittel. Dabei ist entscheidend in welchem Maße z.B. Kapillarporen reduziert werden können, um das Schadenspotential der chemischen Lösung durch einen Flüssigwassertransport zu minimieren.
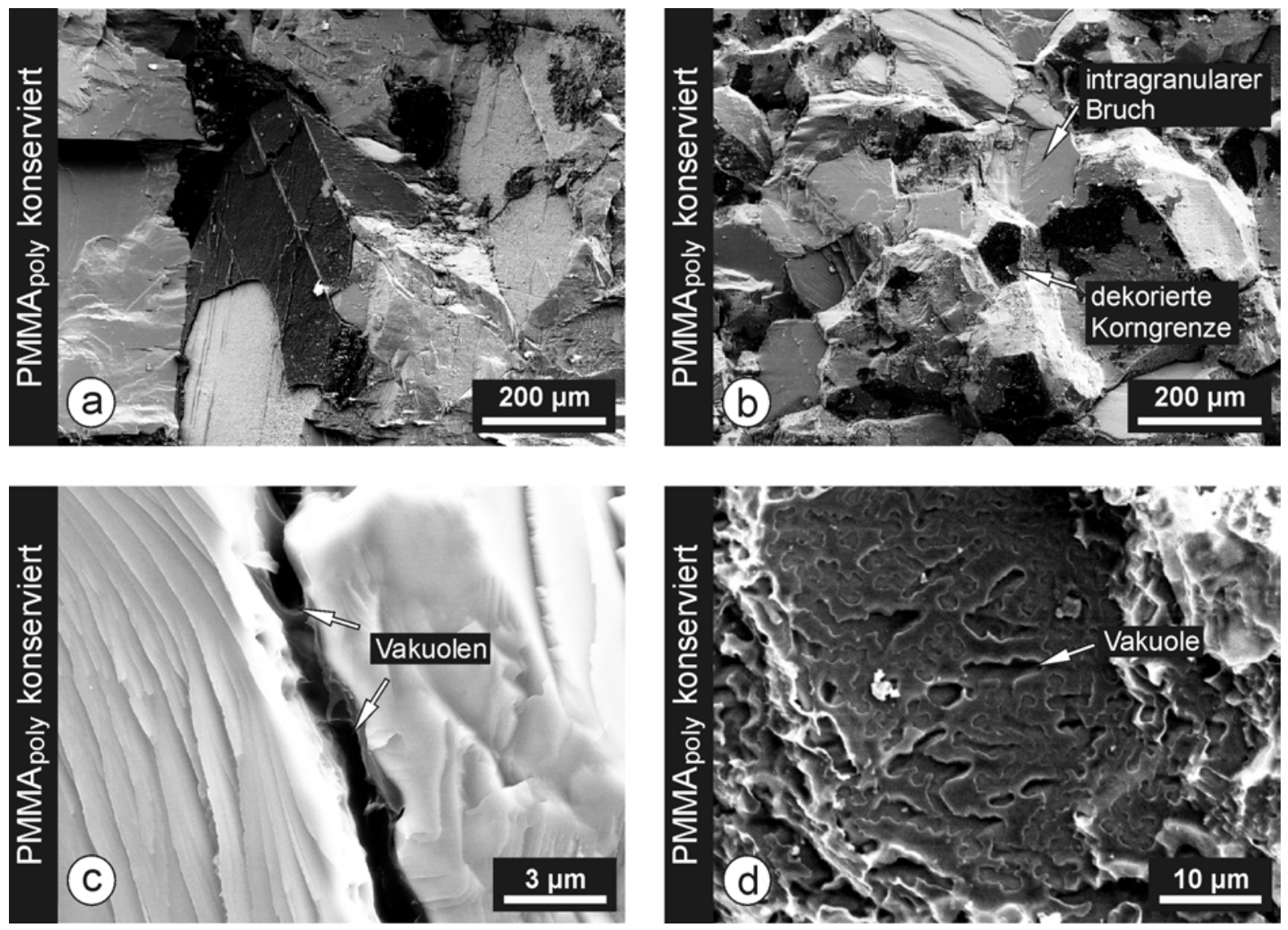

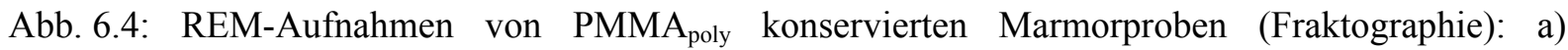
PMMA $_{\text {poly }}$-Filme mit deutlichem Relief des abgerissenen Korns, b) häufige Aktivierung neuer intragranularer Brüche eines Carrara Marmors, der nicht konserviert nur an Korngrenzen entfestigt, c) isolierte Vakuolen im Kunststofffilm und d) abgerissener Kunststofffilm mit freigelegten, amöboid ausgebildeten Vakuolen.

Die mittels $\underline{P M M A}_{s o l}$ konservierten verwitterten Marmorproben weisen eine deutliche Reduktion der Porosität auf (s. Abb. 6.5). Die Herabsetzung des erreichbaren Porenraums beträgt um $40 \%$ und befindet sich damit im Bereich des prozentualen Anteils der aktiven Inhaltsstoffe der Konservierungslösung. Ausnahme ist der Prieborner Marmor mit einer Reduktion der Porosität von ca. $60 \%$, wobei hier von einer Verschließung von Porenräumen auszugehen ist, welche nicht mehr vom Quecksilber erreicht werden können. Mit der Herabsetzung der Porosität ist auch eine deutliche Veränderung der Porengrößenverteilung verbunden (s. Abb. 6.5). Auffallend ist dabei die Verschiebung der am häufigsten vorkommenden Porengrößen um ein bis zwei Klassen zu niedrigeren Werten. Weiterhin erfolgt eine deutliche Reduzierung kleiner Poren. Die Mehrzahl der Poren befindet sich jedoch auch nach der Imprägnierung oberhalb von $0,1 \mu \mathrm{m}$ Porendurchmesser und damit noch deutlich im Bereich des kapillaren Wassertransports. 

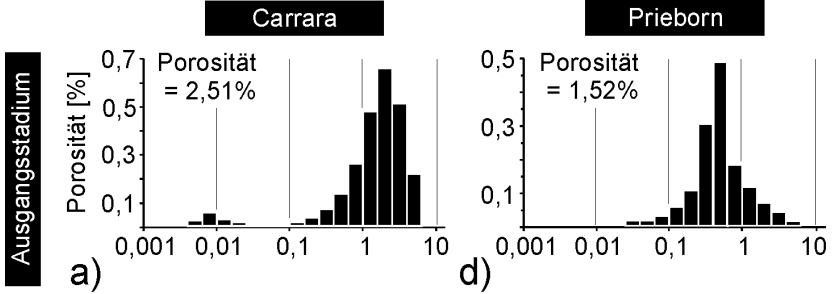

d)
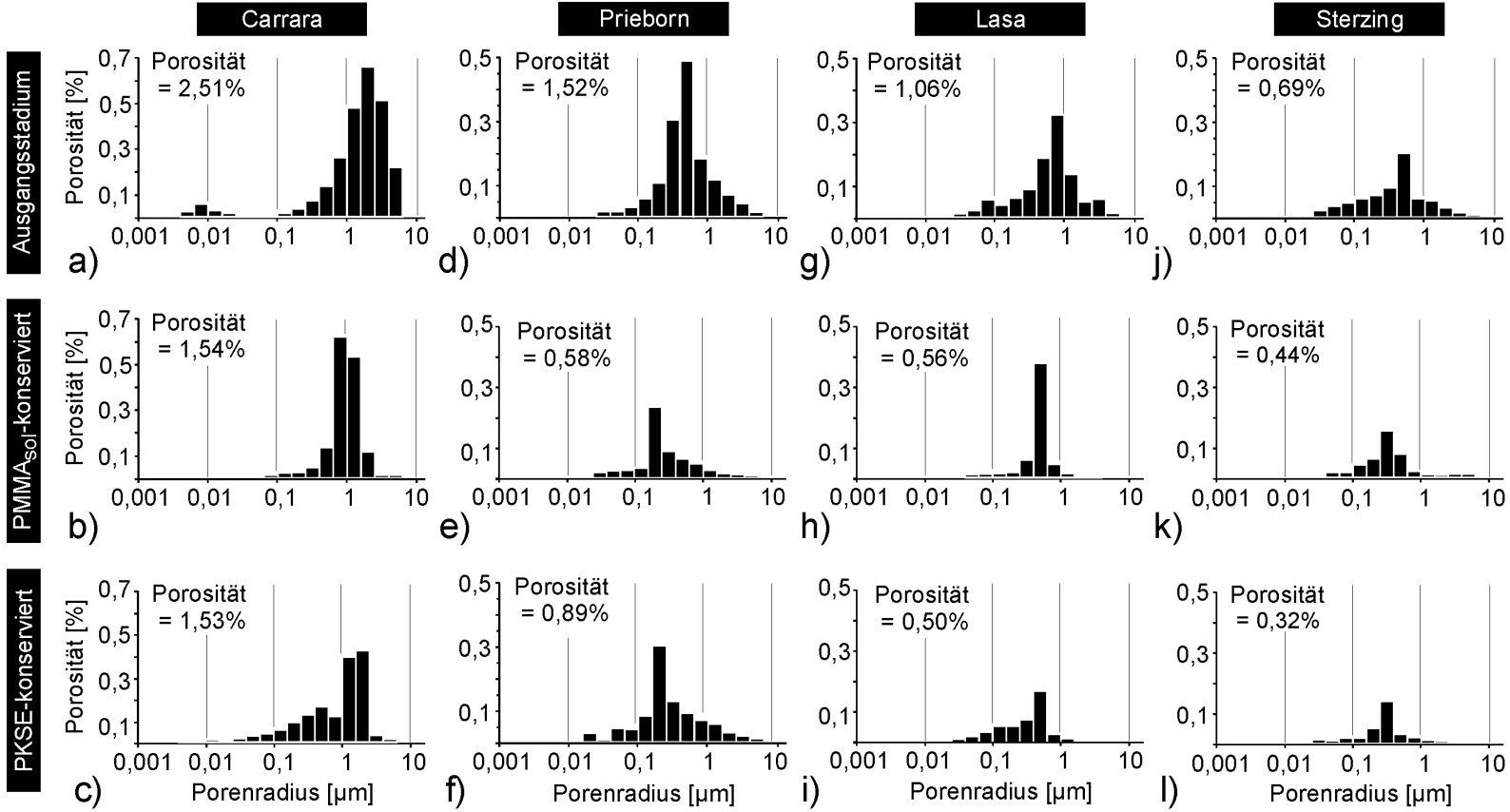

Abb. 6.5: Porositäten und Porengrößenverteilungen ausgewählter Marmortypen im a,d,g,j)

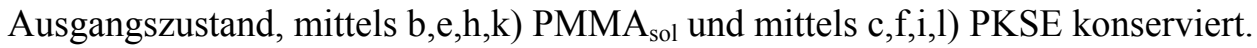

Für die Modifizierung des Porenraums durch die PKSE-Konservierung mit dem angewandten Verfahren ist eine vergleichbare Reduktion der Porosität und der Porengrößenverteilung wie

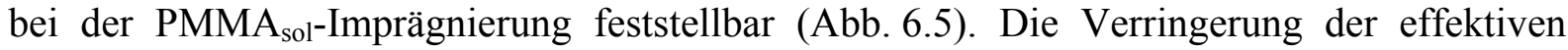
Porosität fällt hierbei etwas höher aus und beträgt für den Laaser und Sterzinger Marmor gut 50 \%. Die Veränderung des Porenradienspektrums ist durch eine Verschiebung der häufigst besetzten Porenradienklasse in eine niedrigere Klasse gekennzeichnet. Im Gegensatz zum

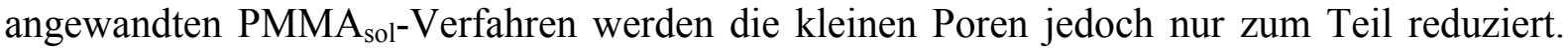
Die Mehrzahl der Poren befindet sich jedoch auch nach der PKSE-Konservierung oberhalb von $0,1 \mu \mathrm{m}$ Porendurchmesser, womit ebenfalls ein ausgeprägter kapillarer Wassertransport zu erwarten ist.

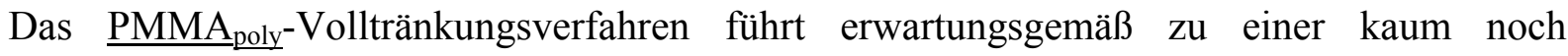
vorhandenen bzw. messbaren Porosität. In Abb. 6.6 ist dies exemplarisch für eine mittels $\mathrm{PMMA}_{\text {poly }}$ imprägnierte Prieborner Marmorprobe dargestellt. Die Porosität ist von 0,96\% der verwitterten Probe auf $0,11 \%$ im konservierten Zustand reduziert worden. Diese experimentell ermittelte Volumenreduktion der Porosität von ungefähr $89 \%$ ist jedoch größer als die verfahrensbedingte Volumenverminderung des Porenraums um ca. 80 \%. Nach REMUntersuchungen weisen die Vakuolen, welche den noch verbliebenen Porenraum repräsentieren, keine Vernetzung untereinander auf. Infolgedessen ist davon auszugehen, dass sie für die Quecksilberporosimetrie nicht erreichbar sind. Bei der gemessenen Porosität und Porengrößenverteilung handelt es sich daher weitgehend um Oberflächeneffekte (Präparationsspuren). Dies bestätigt auch die visuelle Begutachtung der Probe nach der Messung, wobei kein Quecksilber innerhalb der Probe beobachtet werden kann. 
Prieborn

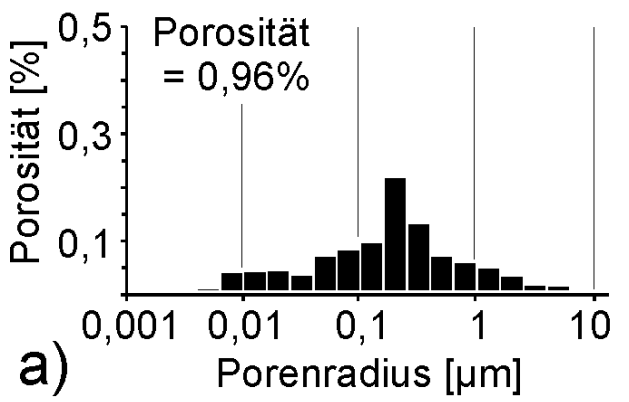

PMMApoly konserviert

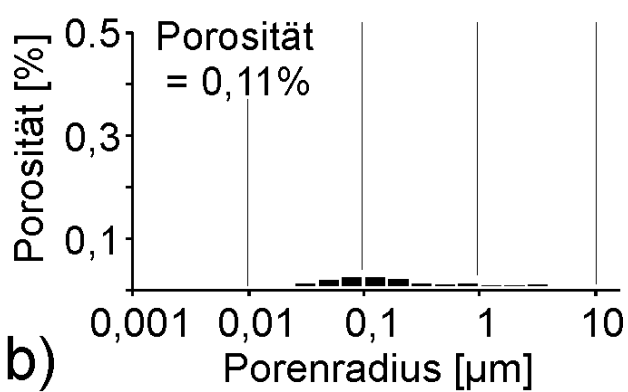

Abb. 6.6: Porositäten und Porengrößenverteilungen einer $\mathrm{PMMA}_{\text {poly }}$ imprägnierten Probe aus Prieborner Marmor: a) Ausgangszustand und b) nach der Konservierung.

\section{- Veränderung der kapillaren Wasseraufnahme}

Die konservierten Marmore zeigen eine deutliche Veränderung der kapillaren Wasseraufnahme. Diese bestätigen die oben aufgeführten Ergebnisse der Porenraumuntersuchungen. So ist für die Acrylharz vollgetränkten Marmore erwatungsgemäß keine kapillare Wasseraufnahme mehr festzustellen. Für die beiden weiteren Konsolidierungsverfahren ist jedoch ein kapillares Aufnahmeverhalten von Wasser noch vorhanden. Dabei ist eine deutliche Variation sowohl für die einzelnen Festiger als auch für die jeweiligen Marmortypen zu ermitteln. Dies ist in Abb. 6.7 exemplarisch für den Carrara und Grosskunzendorfer Marmor dargestellt.

Für die $\underline{\text { PMMA }}_{s o l}$ imprägnierten Proben ist eine ausgeprägte Reduzierung der aufgenommenen Wassermenge festzustellen(Abb. 6.7b,e). Eine starke Herabsetzung ist dabei für die Probe aus Carrara Marmor zu ermitteln (Abb. 6.7b), welche nur noch ungefähr $15 \%$ ihrer im verwitterten Zustand absorbierten Wassermenge aufnimmt. Das Maß der Reduktion ist dabei nicht allein auf die Veränderung des Porengrößenspektrums zurückzuführen (vgl. Abb. 6.5a,b). Möglicherweise handelt es sich hierbei zusätzlich um einen hydrophobierenden Effekt des verwendeten PMMAs.

Für die PKSE konservierten Marmorproben ist hingegen nur eine geringe Abnahme der kapillaren Wasseraufnahme zu beobachten (Abb.6.7c,f). Auffällig ist das Verhalten der Carrara Probe, welche nach der Konservierung eine deutliche Richtungsabhängigkeit aufweist, die im Ausgangszustand nicht vorhanden war. Hierbei bezieht sich die Anisotropie sowohl auf die aufgenommene Menge Wasser in der jeweiligen Richtung als auch auf die Geschwindigkeit der Wasseraufnahme. Dabei ist die kapillare Aufnahme des Wassers in die Z-Richtung schon nach gut 60 min abgeschlossen, wohingegen der imprägnierte Stein in die Y-Richtung bis zu $180 \mathrm{~min}$ benötigt bis dieses Stadium erreicht ist. Resultierend ist festzustellen, dass die Wasseraufnahme einer sich im Ausgangszustand isotrop verhaltenden Probe, nach der Imprägnierung eine Richtungsabhängigkeit aufweisen kann. 

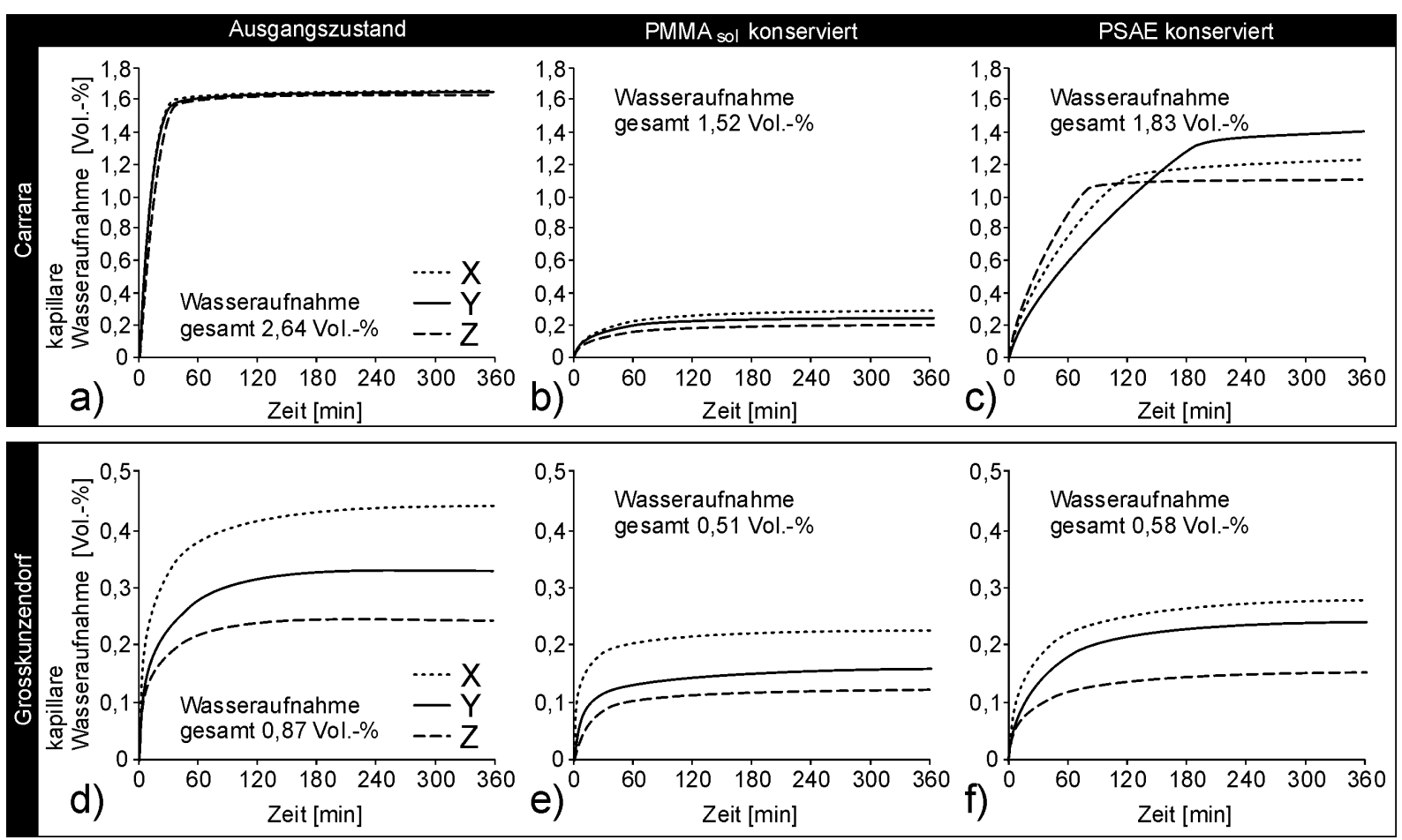

Abb. 6.7: Veränderung der kapillaren Wasseraufnahme konservierter Proben aus Carrara und Grosskunzendorfer Marmor: a,d) Ausgangszustand von Würfelproben (Kantenlänge $65 \mathrm{~mm}$ ), b,e) mittels PMMA sol $_{\text {und }}$ c,f) PKSE konservierte Marmore (b,c,e,f Außenflächen abgesägt, Kantenlänge $60 \mathrm{~mm}$ ).

\section{- Zunahme von Ultraschallgeschwindigkeiten}

Die Zunahme der Ultraschallgeschwindigkeiten nach einer Konservierung verwitterter Marmore erlaubt Aussagen über das Maß der Wiederherstellung bzw. Verbesserung der Kohäsion des Gefügeverbandes durch das jeweilige Verfahren (SNETHLAGE et al., 1999; RUEDRICH et al., 2002). Dabei ist für alle Konservierungsverfahren eine Zunahme der Kompressionswellengeschwindigkeiten festzustellen. Im Detail fallen die Geschwindigkeitsanhebungen jedoch sehr unterschiedlich aus, was zum einen mit der Effektivität des jeweiligen Konservierungsverfahrens zum anderen auf die unterschiedlichen Ausgangszustände vor der Imprägnierung zurückzuführen ist. Die jeweiligen Geschwindigkeitsänderungen für die verschiedenen Konservierungsverfahren, ermittelt durch Messungen an Würfelproben, sind in Tab. 6.1 in $\mathrm{V}_{\text {pmax }}-$ und $\mathrm{V}_{\text {pmin }}$-Richtung für ausgewählte Marmore aufgeführt.

Die mittels $\underline{\text { PMMA }}_{\underline{s o l}}$ gefestigten Proben weisen eine deutliche Anhebung der Ultraschallgeschwindigkeit auf. Diese liegt vielfach über $2 \mathrm{~km} / \mathrm{s}$, wobei maximal eine Erhöhung von 2,7 km/s zu ermitteln ist (vgl. Tab. 6.1). Dabei können Endgeschwindigkeiten von bis zu $5,6 \mathrm{~km} / \mathrm{s}$ erreicht werden. Generell ist die $\mathrm{V}_{\mathrm{p}}$-Erhöhung deutlicher ausgeprägt für die Richtung ursprünglich minimaler Geschwindigkeiten. Damit einhergehend ist auch eine Reduzierung der Richtungsabhängigkeit von $\mathrm{V}_{\mathrm{p}}$ für alle mit $\mathrm{PMMA}_{\mathrm{sol}}$ imprägnierten Proben festzustellen. 
Tab. 6.1: Aus Tischmessungen an Würfelproben gewonnene $\mathrm{V}_{\mathrm{p}}$-Daten für die Veränderung durch die verschiedene Konservierungsverfahren. Fettgedruckt sind Differenzen für Proben mit $\mathrm{V}_{\mathrm{p}^{-}}$ Unterschieden von $>2 \mathrm{~km} / \mathrm{s}$.

\begin{tabular}{|c|c|c|c|c|c|c|c|c|c|}
\hline \multirow{2}{*}{$\begin{array}{l}\text { Marmor- } \\
\text { typ }\end{array}$} & \multirow{2}{*}{$\begin{array}{c}\text { Konservierungs- } \\
\text { methode }\end{array}$} & \multicolumn{3}{|c|}{ NICHT KONSERVIERT } & \multicolumn{3}{|c|}{ KONSERVIERT } & \multicolumn{2}{|c|}{ DIFFERENZ } \\
\hline & & $\begin{array}{c}\mathrm{V}_{\mathrm{pmax}} \\
{[\mathrm{km} / \mathrm{s}]}\end{array}$ & $\begin{array}{c}\mathrm{V}_{\mathrm{pmin}} \\
{[\mathrm{km} / \mathrm{s}]}\end{array}$ & $\begin{array}{l}A_{V p} \\
{[\%]}\end{array}$ & $\begin{array}{l}\mathrm{V}_{\mathrm{pmax}} \\
{[\mathrm{km} / \mathrm{s}]}\end{array}$ & $\begin{array}{c}\mathrm{V}_{\mathrm{pmin}} \\
{[\mathrm{km} / \mathrm{s}]}\end{array}$ & $\begin{array}{l}A_{V p} \\
{[\%]}\end{array}$ & $\begin{array}{l}\mathrm{V}_{\mathrm{pmax}} \\
{[\mathrm{km} / \mathrm{s}]}\end{array}$ & $\begin{array}{c}\mathrm{V}_{\mathrm{pmin}} \\
{[\mathrm{km} / \mathrm{s}]}\end{array}$ \\
\hline \multirow{3}{*}{ Carrara } & $\mathrm{PMMA}_{\mathrm{sol}}$ & 2,4 & 1,9 & 17,4 & 4,8 & 4,1 & 15,4 & 2,5 & 2,1 \\
\hline & PKSE & 2,3 & 1,3 & 44,4 & 2,5 & 1,6 & 35,9 & 0,2 & 0,3 \\
\hline & PMMA $_{\text {poly }}$ & 2,5 & 1,6 & 35,4 & 6,0 & 5,8 & 2,5 & 3,5 & 4,2 \\
\hline \multirow{3}{*}{ Prieborn } & $\mathrm{PMMA}_{\mathrm{sol}}$ & 2,9 & 2,1 & 27,6 & 5,6 & 4,9 & 13,4 & 2,7 & 2,7 \\
\hline & PKSE & 3,0 & 2,2 & 26,6 & 4,5 & 4,0 & 11,4 & 1,4 & 1,7 \\
\hline & PMMA $_{\text {poly }}$ & 4,7 & 3,5 & 26,0 & 6,0 & 5,4 & 9,3 & 1,3 & 2,0 \\
\hline \multirow{3}{*}{ Lasa } & $\mathrm{PMMA}_{\text {sol }}$ & 3,1 & 2,5 & 21,0 & 5,5 & 4,9 & 10,3 & 2,4 & 2,4 \\
\hline & PKSE & 3,0 & 2,4 & 20,0 & 4,3 & 3,9 & 10,6 & 1,3 & 1,5 \\
\hline & PMMA $_{\text {poly }}$ & 4,5 & 3,8 & 15,8 & 5,9 & 5,5 & 6,8 & 1,4 & 1,7 \\
\hline \multirow{3}{*}{ Sterzing } & $\mathrm{PMMA}_{\mathrm{sol}}$ & 3,6 & 3,1 & 14,1 & 5,3 & 5,1 & 3,8 & 1,7 & 2,0 \\
\hline & PKSE & 3,5 & 3,0 & 14,1 & 4,5 & 4,2 & 7,3 & 1,0 & 1,2 \\
\hline & PMMA $_{\text {poly }}$ & 5,0 & 4,5 & 9,2 & 5,8 & 5,5 & 5,0 & 0,8 & 1,0 \\
\hline
\end{tabular}

Auch die PKSE konservierten Proben zeigen einen Anstieg von $V_{p}$, der jedoch gegenüber dem PMMA $_{\text {sol }}$-Verfahren deutlich geringer ausfällt. Dabei können zumeist $\mathrm{V}_{\mathrm{p}}$-Anstiege zwischen $1,0 \mathrm{~km} / \mathrm{s}$ und 1,7 km/s beobachtet werden (vgl. Tab. 6.1). In der Regel erreichen die PKSE konservierten Proben Geschwindigkeiten von bis $\mathrm{zu} 4,5 \mathrm{~km} / \mathrm{s}$. Ausnahme ist die geringe $\mathrm{V}_{\mathrm{p}}$ - Änderung der stark verwitterten Carrara Probe, bei der nur eine Zunahme der Geschwindigkeiten von $0,2 \mathrm{~km} / \mathrm{s}$ bzw. $0,3 \mathrm{~km} / \mathrm{s}$ auf insgesamt 2,5km/s bzw. $1,6 \mathrm{~km} / \mathrm{s}$ ermittelbar ist. Die z.T. geringen Geschwindigkeitsänderungen resultieren aus der eingeschränkten Anhaftung des Kieselsäureesters auf den Calcit-Oberflächen und der damit verbundenen schwachen elastischen Ankopplung.

Die mittels $\underline{P M M A}_{\text {poly }}$ vollgetränkten Proben erreichen erwartungsgemäß sehr hohe Ultraschallgeschwindigkeiten um $6,0 \mathrm{~km} / \mathrm{s}$ (s. Tab. 6.1). Dies sind Geschwindigkeiten, die auch für wassergesättigte Proben gemessen werden können, d.h. der Einfluss der Mikrorisse ist weitgehend minimiert. Die absolute Anhebung der Geschwindigkeiten fällt im Verhältnis $\mathrm{zu}$ den beiden anderen Konservierungsverfahren jedoch geringer aus. Dies ist auf den unterschiedlichen Ausgangszustand der Proben vor der Tränkung zurückzuführen. Nur die stark verwitterte Probe aus Carrara Marmor besitzt annähernd den gleichen Erhaltungszustand vor den modellhaften Konservierungen, weshalb hier ein direkter Vergleich möglich ist. Dabei zeigt das Volltränkungsverfahren mit Abstand die größte Erhöhung von 3,5 km/s bzw. 4,2 km/s (vgl. Tab. 6.1). Generell wird durch die Tränkung auch die Anisotropie herabgesetzt.

Zum Vergleich der Geschwindigkeitsverteilungen in Gesteinskugeln vor und nach einer Tränkung mittels $\mathrm{PMMA}_{\text {poly }}$ sowie im wassergesättigten und im trockenen Zustand sind die jeweiligen Daten für den Prieborner und den Kauffunger Marmor in Abb. 6.8 dargestellt. Dabei sollte der wassergesättigte, nicht imprägnierte Zustand annähernd die intrinsischen Verhältnisse sowohl der absoluten Geschwindigkeiten als auch der Verteilung von $V_{p}$ 
widerspiegeln (Abb. 6.8; vgl. Kap. 4.3.3.3). Abweichend davon ist die Geschwindigkeitsverteilung der nicht imprägnierten Proben im trockenen Zustand (Abb. 6.8b,g), was auf die entsprechende Modifikation durch Rissbildung zurückzuführen ist. Durch die Imprägnierung mittels PMMA $_{\text {poly }}$ werden die Geschwindigkeiten erhöht und erreichen diejenigen des wassergesättigten Zustands (Abb. 6.8). Ebenfalls gleicht das Muster der Geschwindigkeitsverteilung der des wassergesättigten und damit des intrinsischen Zustands. Daraus ergibt sich, dass die Volltränkung auf die Ultraschallgeschwindigkeitsverteilung eine vergleichbare Auswirkung wie die Wassersättigung besitzt. Nur für die imprägnierte Probe des Prieborner Marmors ist bei zusätzlicher Wassersättigung noch eine geringfügig erhöhte PWellengeschwindigkeit festzustellen (vgl. Abb. 6.8b).

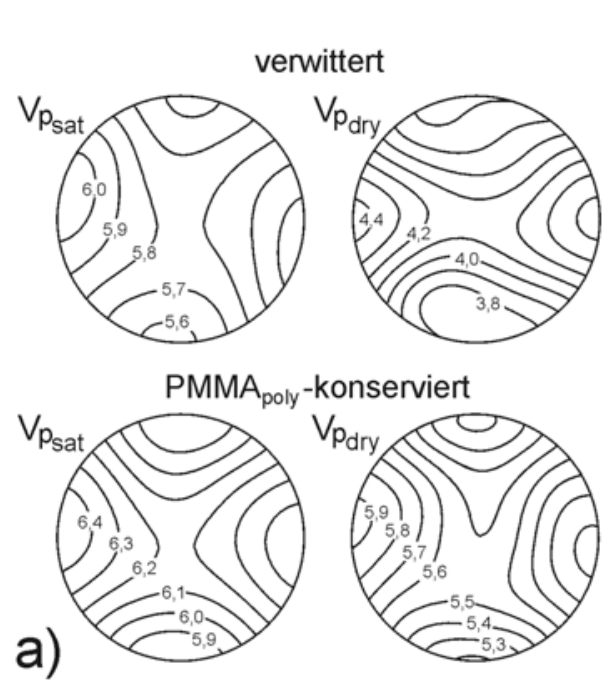

\section{Prieborn}

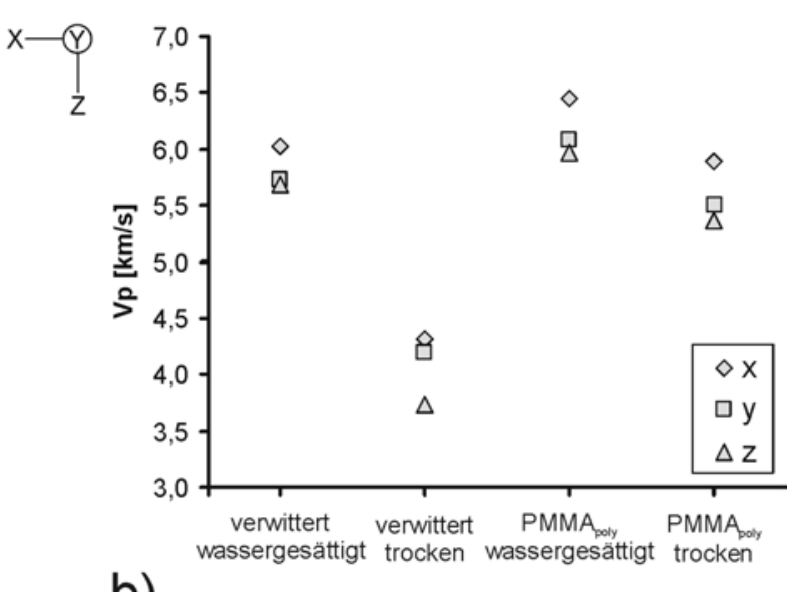

b)

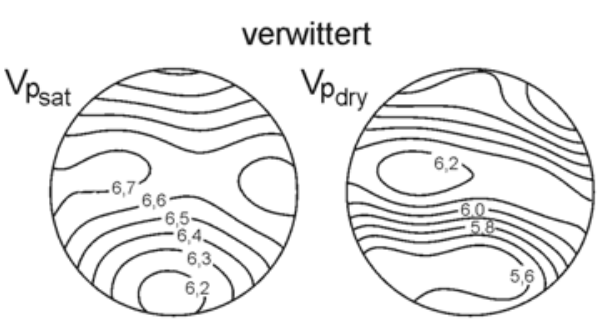

\section{Kauffung}

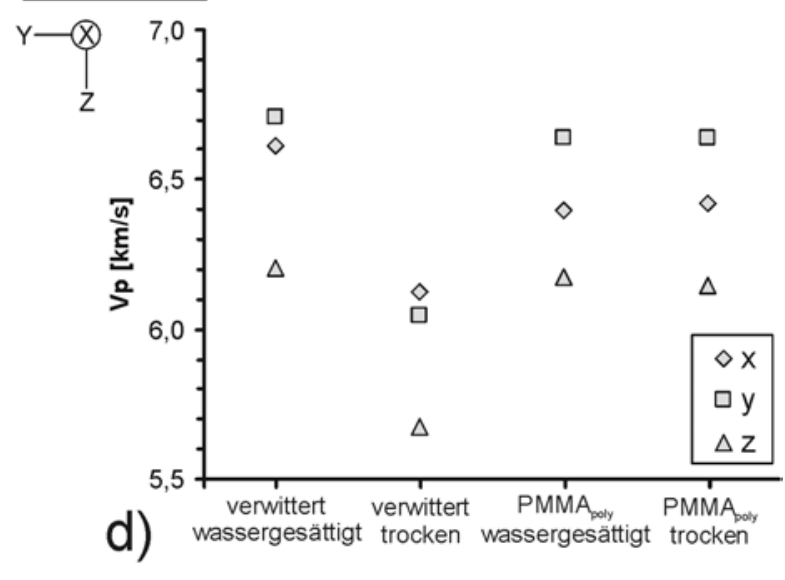

C)

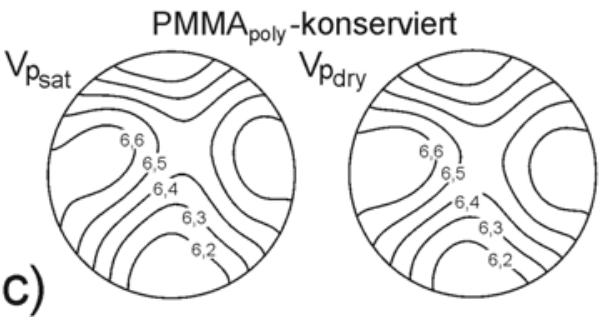

d)

Abb. 6.8: Änderung der P-Wellengeschwindigkeitsverteilung durch Konservierung mit $\mathrm{PMMA}_{\text {poly }}$ für Kugelproben des Prieborner und Kauffunger Marmor: a,c) Darstellung der Geschwindigkeitsverteilung der nicht-konservierten und konservierten Proben im trockenen $\left(\mathrm{V}_{\text {pdry }}\right)$ und wassergesättigten $\left(\mathrm{V}_{\mathrm{psat}}\right)$ Zustand (stereographische Projektion, untere Halbkugel), b,d) Diagramm der Geschwindigkeitsänderung für die Hauptgefügerichtungen. 


\section{- Zunahme der Spaltzugfestigkeit}

Durchzuführende Konservierungsmaßnahmen zielen in den meisten Fällen auf eine Festigung, d.h. die Wiederherstellung bzw. Erhöhung der Kohäsion des entfestigten Naturwerksteins ab. Mit der Verbesserung des Gefügezusammenhalts ist auch eine Zunahme der Zugfestigkeiten verbunden. Der Grad der Festigkeitserhöhung stellt dabei ein Maß für die Zunahme der Kohäsion für Naturwerksteine dar. Damit erlaubt der Vergleich von Spaltzugfestigkeiten vor und nach einer Konservierung eine Aussage über die festigende Wirkung der durchgeführten Maßnahme.

Um die Festigkeitserhöhung für die durchgeführten modellhaften Konservierungen zu charakterisieren, wurden Messungen der Spaltzugfestigkeiten an den zuvor richtungsabhängig charakterisierten Marmortypen aus Sterzing und Carrara nach einer Imprägnierung durchgeführt. Zum Vergleich des Ausgangszustandes wurden die Daten der auf $200{ }^{\circ} \mathrm{C}$ vorkonditionierten Marmorproben für die $\mathrm{PMMA}_{\text {sol }}$ und PKSE Imprägnierung und für die $\mathrm{PMMA}_{\text {poly }}$ Konservierung die Daten der verwitterten Marmorproben herangezogen. Dabei ist für alle angewandten Konservierungsverfahren eine Erhöhung der Festigkeitswerte gegenüber dem Ausgangszustand festzustellen.

Die mittels $\underline{\text { PMMA }}_{\underline{s o l}}$ konservierten Proben aus Carrara und Sterzinger Marmor zeigen einen ungefähr vergleichbaren und deutlichen Anstieg der Spaltzugfestigkeiten (Abb. 6.9). Hierbei ist für den Carrara Marmor eine Zunahme $\Delta \sigma_{z}=0,72 \mathrm{MPa}$ auf $\sigma_{z_{-} \max }=6,26 \mathrm{MPa}$ und von $\Delta \sigma_{z}=1,68 \mathrm{MPa}$ auf $\sigma_{z_{-} \min }=4,81 \mathrm{MPa} \mathrm{zu}$ ermitteln. Der Sterzinger Marmor zeigt einen Festigkeitsanstieg von $\Delta \sigma_{\mathrm{z}}=1,58 \mathrm{MPa}$ auf $\sigma_{z_{-} \max }=5,88 \mathrm{MPa}$ und $\Delta \sigma_{\mathrm{z}}=0,93 \mathrm{MPa}$ auf $\sigma_{z_{-} \min }=4,02 \mathrm{MPa}$. Unterschiedlich ist für beide Proben die Richtung der maximalen bzw. minimalen Erhöhung der Festigkeitswerte. So ist für den Sterzinger Marmor festzustellen, dass eine deutlichere Erhöhung in die Richtung maximaler Spaltzugfestigkeit erfolgt und damit die Richtungsabhängigkeit weiter verstärkt wird. Im Gegensatz dazu zeigt der Carrara Marmor eine stärkere Festigkeitszunahme, in die Richtung der ursprünglich minimalen Werte, womit eine Abnahme der Festigkeitsanisotropie einhergeht.

Die PKSE konservierten Proben weisen ebenfalls eine deutliche Erhöhung der Spaltzugfestigkeiten auf (vgl. ROHATSCH et al., 2000). Diese erhöht sich für den Carrara Marmor in der maximal Richtung mit $\Delta \sigma_{\mathrm{z}}=0,51 \mathrm{MPa}$ auf $\sigma_{z_{-} \max }=6,05 \mathrm{MPa}$ und in die Richtung minimaler Festigkeit um $\Delta \sigma_{z_{-} \min } 1,90 \mathrm{MPa}$ auf $\sigma_{z_{-} \min }=4,46 \mathrm{MPa}$ (Abb. 6.9a). Für den Sterzinger Marmor liegt die Erhöhung bei $\Delta \sigma_{z} 1,47 \mathrm{MPa}$ und erhöht sich auf $\sigma_{z_{-} \max }=5,77 \mathrm{MPa}$ und in die Richtung minimaler Festigkeit bei $\Delta \sigma_{z_{-} \min } 0,61 \mathrm{MPa}$ auf $\Delta \sigma_{z_{-} \min } 3,70 \mathrm{MPa}$ (Abb. 6.9b). Dabei ist ein entsprechendes Verhalten der Änderung der

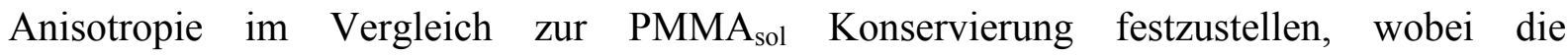
Richtungsabhängigkeit des Carrara Marmor abnimmt und sich die des Sterzinger Marmors durch die PKSE-Festigung erhöht.

Aufgrund der fehlenden oder nur geringen Oberflächenhaftung des PKSE auf Calcitkristallen ist eigentlich nicht mit einer so deutlichen Erhöhung der Zugfestigkeiten zu rechnen (vgl. GoINS et al., 1996). Eine mögliche Erklärung ist die partielle Porenfüllung und eine damit verbundene Stabilisierung des Korngerüsts. Diese könnte insbesondere im Bereich der 
Druckspannungen also, in der Nähe der Kraftübertragungsschwerter, einen Einfluss auf die Materialeigenschaften ausüben.
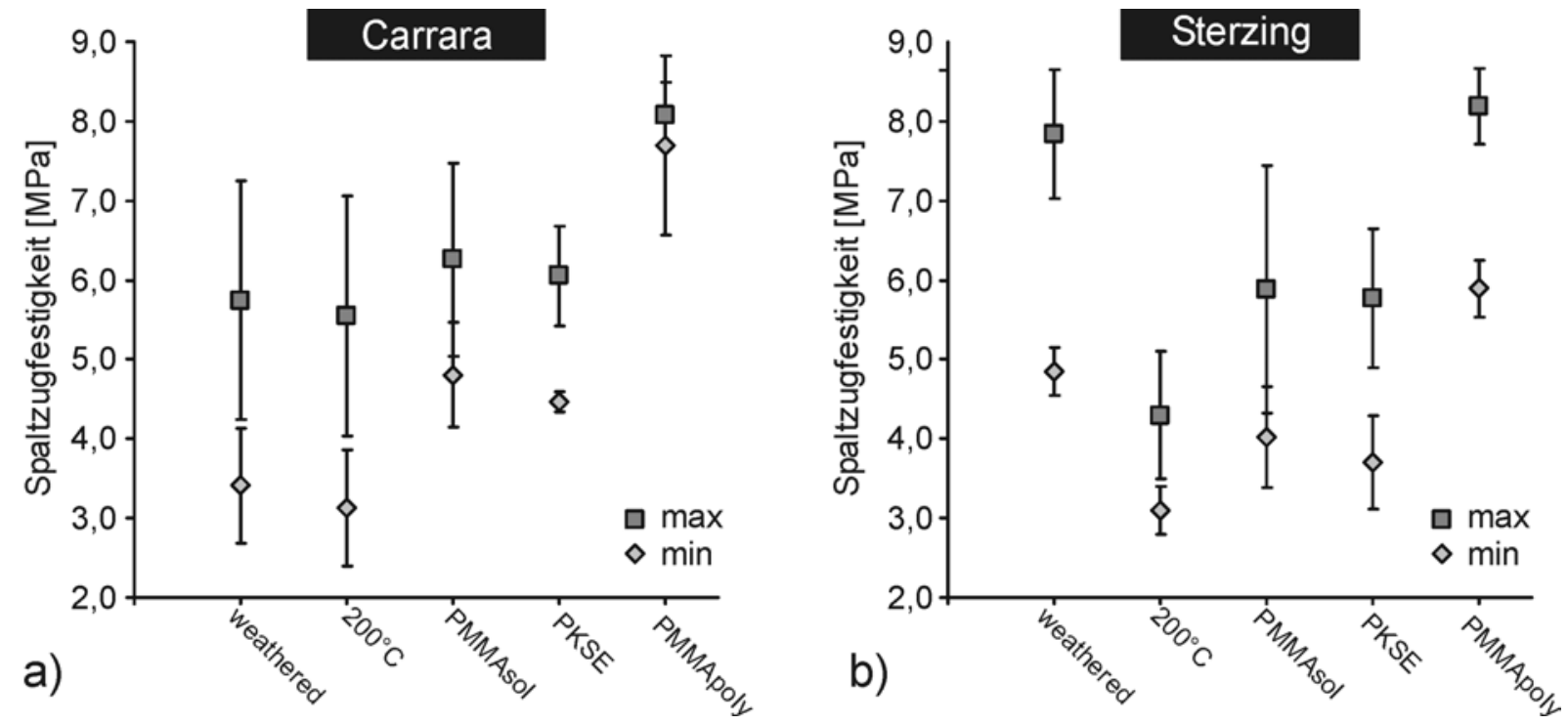

Abb. 6.9: Mittels Brazil-Test ermittelte Spaltzugfestigkeiten des a) Carrara und des b) Sterzinger Marmors in verschiedenen Probenzuständen (verwittert, beansprucht auf $200 \mathrm{C}$, konserviert mittels PMMA sol $_{\text {, PKSE und PMMA }}$ poly).

Die $\underline{\text { PMMA }}_{\text {poly }}$-Volltränkung führt zum stärksten Anstieg der Spaltzugfestigkeiten, die sich im Bereich unverwitterter Marmore befinden. Dabei erreicht der Carrara Marmor sowohl für die minimale als auch für die maximale Richtung Werte um 8,00 MPa (Abb. 6.9a). Dies bedeutet eine Zunahme der Spaltzugfestigkeit von $\Delta \sigma_{z_{-} \max } 4,29 \mathrm{MPa}$ und $\Delta \sigma_{z_{-} \min } 2,34 \mathrm{MPa}$. Für den Sterzinger Marmor ist eine deutlich geringere Festigkeitszunahme mit $\Delta \sigma_{z_{-} \max } 0,35 \mathrm{MPa}$ und $\Delta \sigma_{z_{-} \min } 1,05 \mathrm{MPa}$ zu ermitteln (Abb. 6.9b). Er erreicht jedoch ebenfalls recht hohe Festigkeiten von maximal gut 8,00 MPa und minimal knapp 6,00 MPa.

Auffallend für beide Marmore ist das unterschiedliche Verhalten der Anisotropie der konservierten Proben. Nach REM-Untersuchungen verläuft der Bruch im Carrara Marmor (Korngefügetyp I) bevorzugt entlang neugebildeter, intrakristalliner Flächen. Damit sollte die für die starke Richtungsabhängigkeit im Ausgangszustand verantwortlich gemachte Kornformregelung nach der Volltränkung eine geringere Bedeutung besitzen. Dies würde die nur sehr schwache Anisotropie der gefestigten Probe erklären. Hingegen zeigt der Sterzinger Marmor (Korngefügetyp II) nach wie vor die Aktivierung sowohl gefestigter als auch neugebildeter intragranularer Risse. Hier sollte weiterhin die Textur für die starke Richtungsabhängigkeit der gefestigten Probe verantwortlich sein. 


\section{Verhalten konservierter Marmore bei thermischer Beanspruchung}

Obwohl umfangreiche Informationen über das Materialverhalten von Naturwerksteinen als auch von gängigen Konservierungsmitteln existieren, wurden bislang nur wenige Untersuchungen, welche die Veränderung der Eigenschaften eines Gesteins nach einer Konservierungsmaßname beschreiben, durchgeführt. Bezüglich eines konservierten Marmors stehen dabei Fragen im Vordergrund wie z.B., ob ein Festiger überhaupt mit der mineralogischen Zusammensetzung, dem Gefüge und den petrophysikalischen Eigenschaften eines Gesteins kompatibel ist oder welchen Einfluss ein Konservierungsmittel auf die materialspezifischen Verwitterungsprozesse hat.

Um zu überprüfen, in welcher Art und in welchem Umfang die thermisch bedingte Gefügeentfestigung von Marmoren durch das jeweilige Konservierungsverfahren beeinflusst oder verändert wird, wurde das thermische Längenänderungsverhalten der zuvor imprägnierten Proben ermittelt. Die Überprüfung einer möglichen Gefügeentfestigung erfolgte mittels Ultraschallanalysen vor und nach einer bestimmten thermischen Beanspruchung.

\subsection{Thermische Längenänderung}

Um das thermische Verwitterungsverhalten von konservierten Marmoren zu charakterisieren, wurden die Dehnungseigenschaften der Gesteine vor und nach einer Imprägnierung miteinander verglichen. Dazu erfolgten Messungen der thermischen Längenänderung sowie der Ermittlung der entsprechenden Restdehnungen durch zyklisches Erwärmen auf Temperaturstufen von $42{ }^{\circ} \mathrm{C}, 65^{\circ} \mathrm{C}, 90^{\circ} \mathrm{C}$ sowie wiederholtes Heizen auf $90{ }^{\circ} \mathrm{C}$. Die Ergebnisse zeigen, dass die durchgeführten Konservierungen von Marmorproben in signifikanten, verfahrensspezifischen Veränderungen des thermischen Dehnungsverhaltens resultieren. Im folgenden Abschnitt ist das Längenänderungsverhalten für ausgewählte konservierte Marmore in Bezug zu den verschiedenen Heizrampen zusammengefasst. Entsprechende Dehnungskurven sind in Abb. 7.1 und Abb. 7.2 dargestellt, und Daten der permanenten Längenänderung befinden sich in Tab 7.1.

\section{- PMMA $A_{\text {sol }}$ konservierte Marmore}

Von den mittels PMMA $_{\text {sol }}$ konservierten Marmoren weisen die meisten Proben beim Erwärmen auf $42^{\circ} \mathrm{C}$ eine schwach gekrümmte Expansionkurve auf. Entsprechend ist beim Abkühlen auf den Ausgangszustand keine messbare Restdehnung zu verzeichnen (Abb. 7.1a). Ausnahmen sind die Z-Richtung des Carrara und die Y-Richtung des Prieborner Marmors, wobei hier nur eine geringe aber doch messbare Restdehnung nach entsprechender thermischer Beanspruchung auftritt (s. Tab. 7.1). 
Tab. 7.1: Experimentell ermittelte Restdehnungen $(\Delta \varepsilon)$ in die Hauptgefügerichtungen für die Heizzyklen $42{ }^{\circ} \mathrm{C}, 65^{\circ} \mathrm{C}, 90^{\circ} \mathrm{C}$ und wiederholtem Heizzyklus auf $90{ }^{\circ} \mathrm{C}$ ausgewählter verwitterter und konservierter Marmore. Werte unter $0,020 \mathrm{~mm} / \mathrm{m}$ wurden gleich null gesetzt (unterhalb der gerätetechnischen Auflösung) und Werte über $0,050 \mathrm{~mm} / \mathrm{m}$ sind fett gedruckt (ausgeprägte Restdehnung).

\begin{tabular}{|c|c|c|c|c|c|c|}
\hline $\begin{array}{l}\text { Marmor- } \\
\text { typ }\end{array}$ & Zustand & Richtung & $\begin{array}{c}\text { Zyklus } 42{ }^{\circ} \mathrm{C} \\
\Delta \varepsilon[\mathrm{mm} / \mathrm{m}]\end{array}$ & $\begin{array}{r}\text { Zyklus } 65{ }^{\circ} \mathrm{C} \\
\Delta \varepsilon[\mathrm{mm} / \mathrm{m}]\end{array}$ & $\begin{array}{c}\text { Zyklus } 90^{\circ} \mathrm{C} \\
\Delta \varepsilon[\mathrm{mm} / \mathrm{m}]\end{array}$ & $\begin{array}{c}\text { Zyklus } 90^{\circ} \mathrm{C} \\
\Delta \varepsilon[\mathrm{mm} / \mathrm{m}]\end{array}$ \\
\hline \multirow{12}{*}{ Carrara } & \multirow{3}{*}{ weathered } & $\mathrm{X}$ & 0,000 & 0,000 & 0,000 & 0,000 \\
\hline & & $\mathrm{Y}$ & 0,000 & 0,000 & 0,000 & 0,000 \\
\hline & & $\mathrm{Z}$ & $-0,071$ & $-0,047$ & $-0,055$ & $-0,032$ \\
\hline & \multirow{3}{*}{$\mathrm{PMMA}_{\text {sol }}$} & $\mathrm{X}$ & 0,000 & 0,063 & 0,111 & 0,000 \\
\hline & & Y & 0,000 & 0,108 & $\mathbf{0 , 0 5 7}$ & 0,000 \\
\hline & & Z & 0,040 & 0,225 & 0,181 & 0,000 \\
\hline & \multirow{3}{*}{ PKSE } & $\mathrm{X}$ & 0,021 & 0,027 & 0,049 & 0,000 \\
\hline & & $\mathrm{Y}$ & 0,000 & 0,039 & 0,056 & 0,000 \\
\hline & & Z & 0,000 & 0,039 & 0,059 & 0,000 \\
\hline & \multirow{3}{*}{ PMMA $_{\text {poly }}$} & $\mathrm{X}$ & 0,000 & 0,000 & 0,150 & 0,043 \\
\hline & & $\mathrm{Y}$ & 0,000 & 0,000 & 0,159 & 0,034 \\
\hline & & $\mathrm{Z}$ & 0,000 & 0,000 & 0,102 & 0,000 \\
\hline \multirow{12}{*}{ Prieborn } & \multirow{3}{*}{ weathered } & $\mathrm{X}$ & 0,000 & 0,054 & 0,099 & 0,000 \\
\hline & & $\mathrm{Y}$ & 0,027 & 0,086 & 0,134 & 0,000 \\
\hline & & Z & 0,042 & 0,066 & 0,094 & 0,000 \\
\hline & \multirow{3}{*}{$\mathrm{PMMA}_{\text {sol }}$} & $\mathrm{X}$ & 0,000 & 0,116 & 0,085 & 0,000 \\
\hline & & Y & 0,032 & 0,141 & 0,095 & 0,000 \\
\hline & & Z & 0,000 & 0,074 & 0,030 & 0,000 \\
\hline & \multirow{3}{*}{ PKSE } & $\mathrm{X}$ & 0,000 & 0,027 & 0,047 & 0,000 \\
\hline & & $\mathrm{Y}$ & 0,000 & 0,052 & 0,055 & $-0,026$ \\
\hline & & Z & 0,035 & 0,045 & 0,042 & 0,000 \\
\hline & \multirow{3}{*}{$\mathrm{PMMA}_{\text {poly }}$} & $\mathrm{X}$ & 0,000 & 0,000 & 0,141 & 0,031 \\
\hline & & $\mathrm{Y}$ & 0,000 & 0,000 & 0,185 & 0,026 \\
\hline & & Z & 0,000 & 0,000 & 0,128 & 0,000 \\
\hline
\end{tabular}

Für den Heizzyklus auf $65{ }^{\circ} \mathrm{C}$ ist für die PMMA ${ }_{\text {sol }}$-Proben oberhalb einer Temperatur von ca. $50{ }^{\circ} \mathrm{C}$ ein deutlicher Anstieg der Dehnungskurve und folglich auch des thermischen Ausdehnungskoeffizienten zu ermitteln (Abb. 7.2). Resultierend weisen insbesondere die Proben des Carrara und Prieborner Marmors eine starke Restdehnung nach thermischer Beanspruchung in allen drei Gefügerichtungen auf. Diese befinden sich dabei deutlich über den permanenten Längenänderungen des nicht konservierten Zustandes (s. Tab. 7.1). Dabei ist der starke Dehnungsanstieg zweifelsfrei auf die Erweichung des Acrylharzes beim Überschreiten seiner Glasübergangstemperatur $\left(\mathrm{T}_{\mathrm{g}}=\right.$ ungefähr $\left.60^{\circ} \mathrm{C}\right)$ zurückzuführen. Für die etwas niedrigeren Temperaturen von $50^{\circ} \mathrm{C}$ ist vermutlich eine noch nicht vollständig abgeschlossene Aushärtung des Konservierungsmittels verantwortlich. Als Ursache für die permanenten Restdehnungen ist somit weniger eine Bildung neuer Risse als vielmehr das plastische Verhalten des PMMA in dem entsprechenden Temperaturbereich anzunehmen.

Für den Lasa und den Sterzinger Marmor ist der gleiche Effekt zu beobachten, welcher jedoch, aufgrund schwächerer Probenvorschädigung und somit auch weniger Konservierungsmittel im Porenraum, geringere Intensität aufweist (Abb. 7.2k,o). 

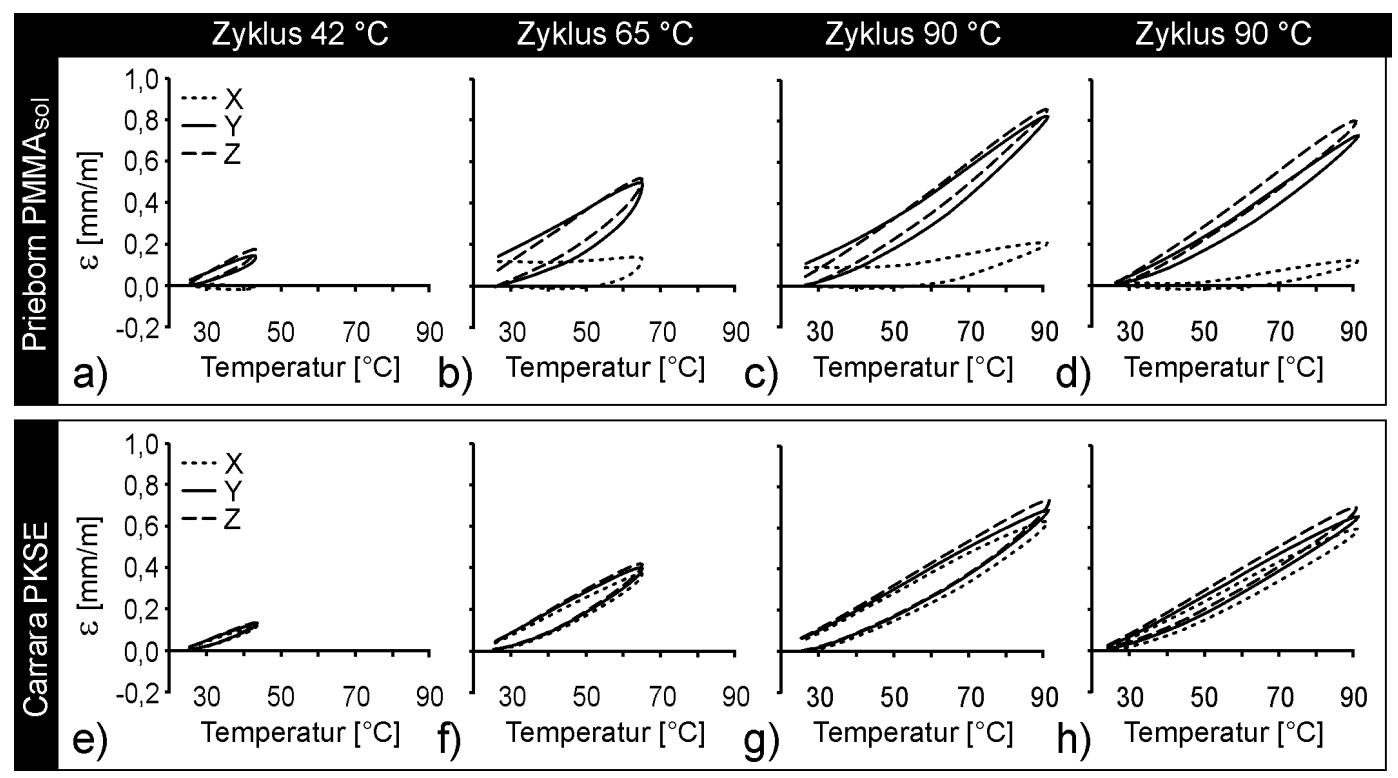

Abb. 7.1: Längenänderungsverhalten von zyklisch auf $42{ }^{\circ} \mathrm{C}, 65^{\circ} \mathrm{C}, 90^{\circ} \mathrm{C}$ und wiederholt auf $90^{\circ} \mathrm{C}$ thermisch beanspruchte Proben a-d) des mit PMMA $\mathrm{A}_{\text {sol }}$ konservierten Prieborner Marmors und e-h) des mit PKSE konservierten Carrara Marmors.

Das thermische Verhalten der Proben beim Erwärmen auf $90{ }^{\circ} \mathrm{C}$ ist gekennzeichnet durch einen leichten Anstieg der Hysteresiskurve oberhalb $65^{\circ} \mathrm{C}$ (vgl. Abb. 7.1c). Eine deutliche Restdehnung ist dabei nur für den Carrara und Prieborner Marmor festzustellen, die jedoch geringer ausfällt als beim Heizzyklus auf $65^{\circ} \mathrm{C}$. Die geringeren permanenten Längenänderungen sind hier ein weiteres Indiz dafür, dass für die starke Dehnungsänderung um $50{ }^{\circ} \mathrm{C}$ die Glasübergangstemperatur ausschlaggebend ist. Für den zweiten Heizzyklus auf $90{ }^{\circ} \mathrm{C}$ ist die Krümmung der Dehnungskurven deutlich geringer ausgeprägt, wobei keine weiteren Restdehnungen zu ermitteln sind (Abb. 7.1d; Tab. 7.1). Somit ist auch für den konservierten Zustand mit $\mathrm{PMMA}_{\text {sol }}$ festzustellen, dass nach Beanspruchung auf eine vormalig erreichte Temperaturstufe, rein thermisch keine weitere Veränderung der Proben auftritt.

Für alle Temperaturstufen ist bezüglich der Richtungsabhängigkeit der Restdehnungen festzustellen, dass Richtungen größter permanenter Längenänderungen mit denen der nichtkonservierten Proben übereinstimmen. Dies bedeutet, dass die Richtungen stärkster Schädigung im Ausgangszustand auch nach einer Festigung wieder bevorzugt aktiviert werden. Ein besonderes Beispiel hierfür ist die sehr stark verwitterte Probe des Carrara Marmors. Im Ausgangszustand weist sie parallel zur Z-Richtung sogar eine ausgeprägte Kontraktion auf, die Ausdruck einer starken Entfestigung ist. Im konservierten Zustand ist dies für alle Heizzyklen auch die Richtung höchster Restdehnungen.

\section{- PKSE konservierte Marmore}

Die PKSE konservierten Proben weisen nur geringe Änderungen des thermischen Längenänderungsverhaltens im Vergleich $\mathrm{zu}$ den nicht konservierten Marmorproben auf (Abb. 7.2). Für den Heizzyklus auf $42{ }^{\circ} \mathrm{C}$ ist die thermische Dehnung mehr oder weniger linear (Abb. 7.1e). Ebenso können keine signifikanten Restdehnungen beobachtet werden. 
Geringe permanente Längenänderungen sind lediglich für die X-Richtung des Carrara und die Z-Richtung des Prieborner Marmors festzustellen (vgl. Tab. 7.1).
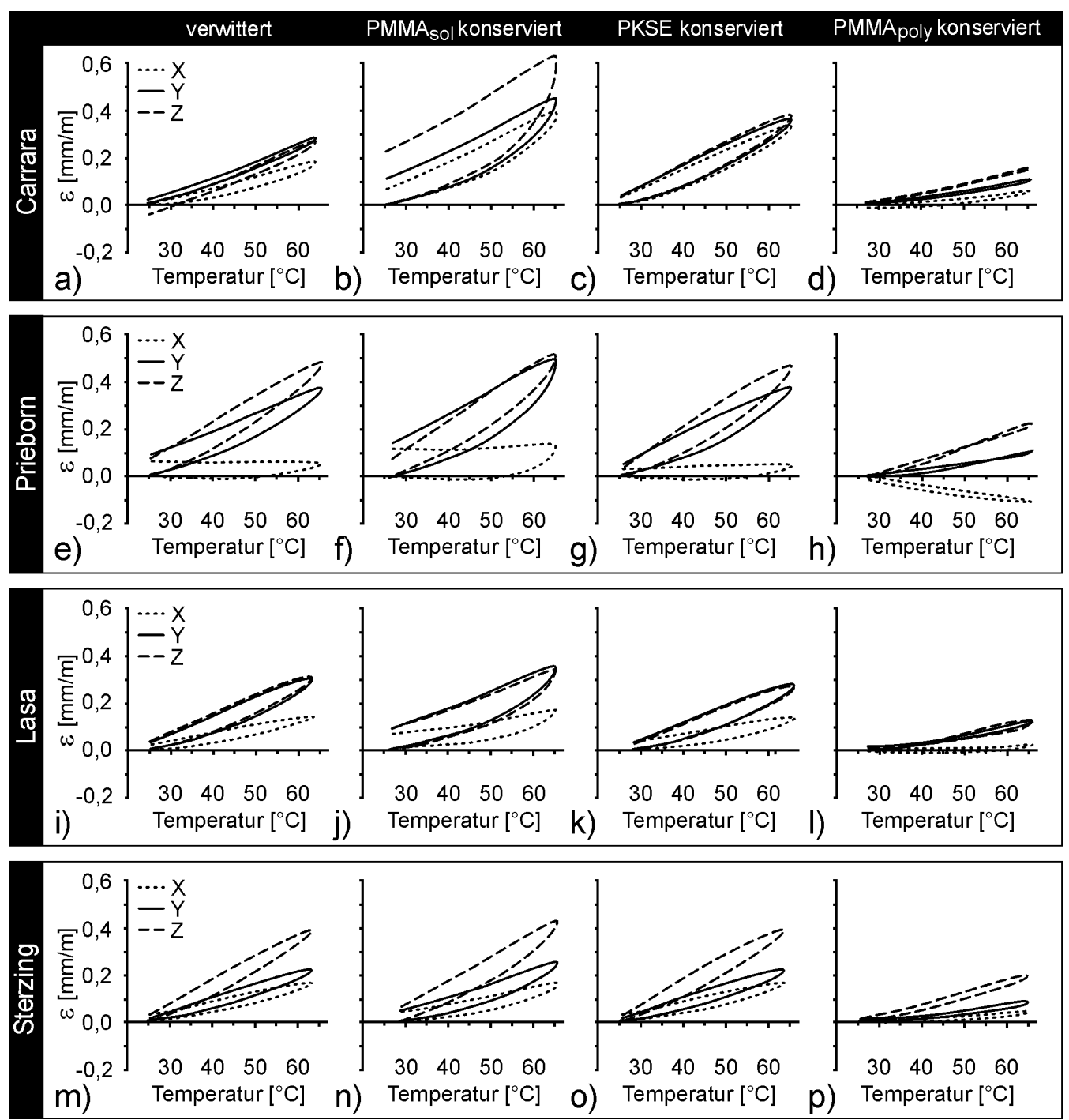

Abb. 7.2: Längenänderungsverhalten von nicht konservierten und konservierten Marmoren bei thermischer Beanspruchung auf $65^{\circ} \mathrm{C}$.

Ebenfalls weicht das Längenänderungsszenario für den Heizzyklus auf $65^{\circ} \mathrm{C}$ und $90{ }^{\circ} \mathrm{C}$ nur geringfügig von dem des nicht-konservierten Zustands ab. Für beide Heizzyklen sind ausgeprägte Hysteresis mit schwachem Dehnungsanstieg sowie geringe bis mittlere Restdehnungsbeträge zu ermitteln (Tab. 7.1). Auffallend ist das Dehnungsverhalten der Carrara Probe, welche im verwitterten Zustand einen Gefügekollaps mit permanenter Kontraktion nach thermischer Beanspruchung aufweist. Dieses Verhalten ist auf die extreme Gefügeentfestigung dieser Marmorprobe zurückzuführen. Hingegen ist für die PKSE imprägnierte Probe ein ,normales“ Dehnungsverhalten mit geringen Restdehnungen zu ermitteln. Diese Beobachtung zeigt, dass das Kieselsäureestergel in der Lage ist, das Korngefüge stark verwitterter Marmore zu stabilisieren. Beim wiederholten Heizen auf $90{ }^{\circ} \mathrm{C}$ verhält sich die Dehnungskurve vergleichbar, wobei keine weiteren permanenten Längenänderungen detektiert werden können. 


\section{- PMMA $_{\text {poly }}$ konservierte Marmore}

Für die Acrylharz-vollgetränkten Marmorproben ist für die Heizzyklen auf $42{ }^{\circ} \mathrm{C}$ und $65^{\circ} \mathrm{C}$ ein sehr ähnliches Dehnungsverhalten zu beobachten, welches jedoch sehr stark von den nicht-konservierten Proben abweicht (s. Abb. 7.2d,h,1,p). Die deutliche Veränderung ist durch eine ausgeprägte Herabsetzung der thermischen Längenänderung gekennzeichnet. Für den Prieborner Marmor ist parallel zur X-Richtung sogar eine deutliche Kontraktion des Gesteins zu ermitteln. Die Kontraktionsrichtung liegt dabei parallel zur bevorzugten Orientierung der kristallographischen a-Achsen. Dieses Kontraktionsverhalten kann auf eine gute Haftung des $\mathrm{PMMA}_{\text {poly }}$ an den Kristalloberflächen zurückgeführt werden, die zu einer besseren Übertragung der Einkristalleigenschaften auf das Gesamtgesteinsverhalten führt (für weitere Erläuterungen s.u.). Weiterhin ist für alle konservierten Proben keine Restdehnung für die Heizzyklen auf $42{ }^{\circ} \mathrm{C}$ und $65^{\circ} \mathrm{C}$ festzustellen (Tab. 7.1).

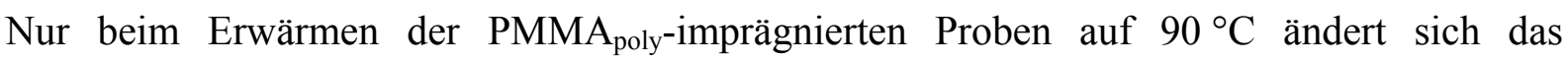
thermische Dehnungsverhalten signifikant. Dieses ist durch einen starken Anstieg der thermischen Dehnung bei über ca. $80^{\circ} \mathrm{C}$ gekennzeichnet (s. Abb. 7.3). Nach dem Abkühlen auf die Ausgangstemperatur verbleibt eine deutliche Restdehnung. Diese experimentell bestimmte Temperatur einer deutlichen Änderung des Dehnungsverhaltens fällt dabei mit der Glasübergangstemperatur $\left(\mathrm{T}_{\mathrm{g}}\right)$ des verwendeten Acrylharzes von ca. $85^{\circ} \mathrm{C}$ zusammen. Damit

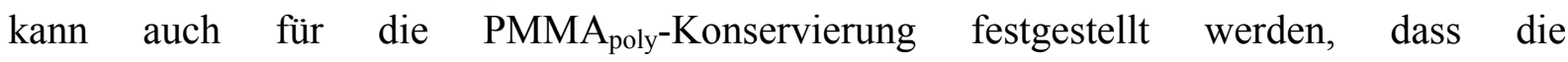
Glasübergangstemperatur einen ausgeprägten Einfluss auf das Dehnungsverhalten eines Marmors beim Erreichen der entsprechenden Temperatur hat.

Für den zweiten Heizzyklus auf $90^{\circ} \mathrm{C}$ ist der Übergangspunkt nur noch sehr schwach ausgebildet (Abb. 7.3). Trotzdem kann eine messbare Restdehnung für die meisten Proben festgestellt werden (s. Tab. 7.1).

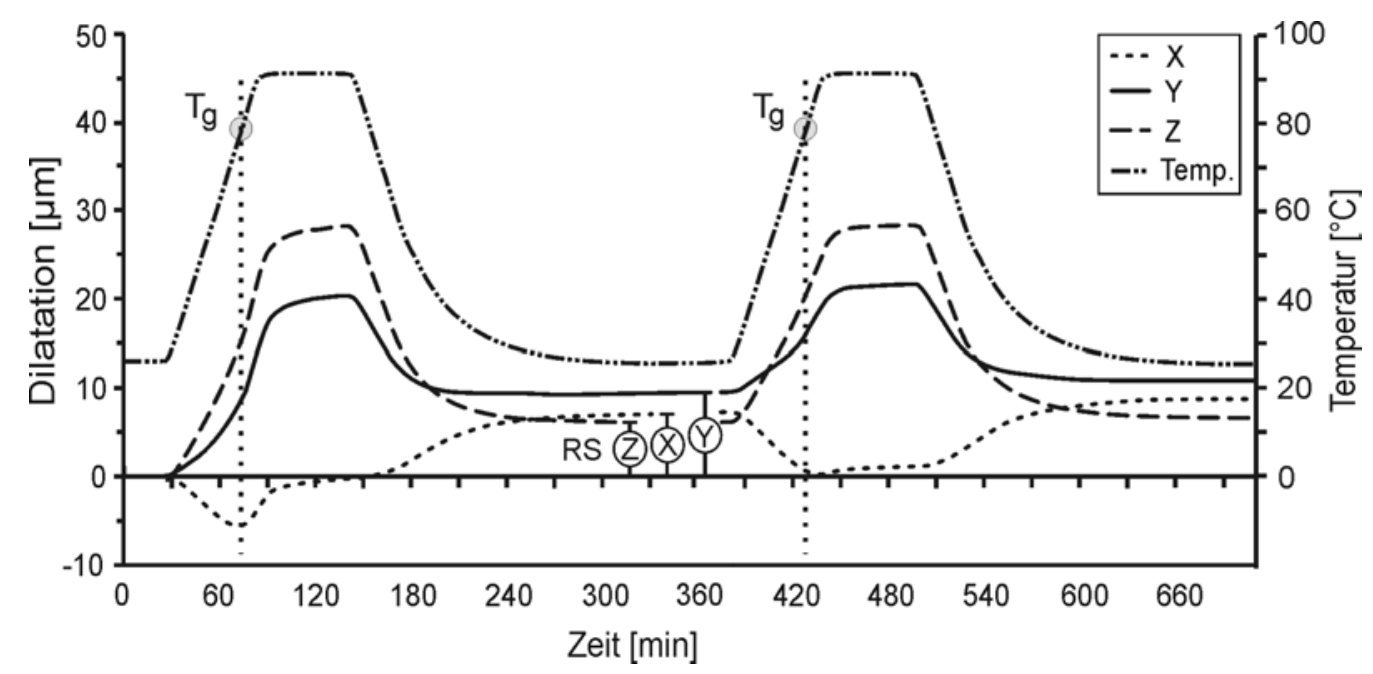

Abb. 7.3: Längenänderungskurven für zwei Heizzyklen auf $90{ }^{\circ} \mathrm{C}$ einer mittels $\mathrm{PMMA}_{\text {poly }}$ konservierten Probe aus Prieborner Marmor. Die jeweilige Restdehnung (RS) ist für alle Richtungen markiert. 


\section{- Änderung des Ausdehnungskoeffizienten für PMMA poly konservierte Marmore}

Die oben festgestellte geringere Ausdehnung nach der Acrylharzvolltränkung drückt sich auch in einer deutlichen Herabsetzung der Ausdehnungskoeffizienten aus. In Abb. 7.4a,b ist zum Vergleich die Entwicklung der Ausdehnungskoeffizienten einer nicht imprägnierten und einer mittels $\mathrm{PMMA}_{\text {poly }}$ konservierten Probe aus Prieborner Marmor bei Beanspruchung bis $65^{\circ} \mathrm{C}$ dargestellt. Die nicht imprägnierte Probe weist einen starken Anstieg der Ausdehnungskoeffizienten auf. In Richtung maximaler Dehnung steigt der Ausdehnungskoeffizient von $\alpha=8 * 10^{-6} \mathrm{~K}^{-1}$ auf ca. $\alpha=13 * 10^{-6} \mathrm{~K}^{-1}$. In Richtung minimaler Längenänderung steigt er von ca. $\alpha=-1,5 * 10^{-6} \mathrm{~K}^{-1}$ auf $\alpha=4 * 10^{-6} \mathrm{~K}^{-1}$. Die imprägnierte Probe zeigt hingegen einen in Richtung maximaler Dehnung annähernd gleich bleibenden Ausdehnungskoeffizienten von ca. $\alpha=5 * 10^{-6} \mathrm{~K}^{-1}$ und in Richtung minimaler Längenänderung einen leicht zunehmend negativen $\alpha$ von $-2,5$ auf $-3 * 10^{-6} \mathrm{~K}^{-1}$.

Dieses Verhalten erscheint zunächst ungewöhnlich, da der Ausdehnungskoeffizient des Acrylharzes gegenüber dem des Calcits deutlich höher liegt. Es wäre zu erwarten, dass sich beide Ausdehnungskoeffizienten addieren, woraus eine geringe Zunahme resultieren würde. Dies kann anhand eines Beispiels nachgerechnet werden. Dazu soll hier der stark verwitterte Carrara Marmor dienen, der vollständige Entfestigung an Korngrenzen aufweist. Er zeigt eine mittlere Korngröße von $170 \mu \mathrm{m}$ und einen mittleren Porengrößendurchmesser von ca. $5 \mu \mathrm{m}$. Bei Annahme einer weit reichenden Ausfüllung der Risse mit Acrylharz sollten statistisch gesehen bei Betrachtung des Materials in einer Richtung der Ausdehnungskoeffizient von 2,9\% Harz und 97,1\% Calcit bedingt sein. Mit Einberechnung eines angenommenen mittleren Ausdehnungskoeffizienten von Calcit bei z.B. $\alpha=13,0^{*} 10^{-6} \mathrm{~K}^{-1}$ (KLEBER, 1959) und dem bekannten des Acrylharz von $\alpha=70,0^{*} 10^{-6} \mathrm{~K}^{-1}$ ergibt sich ein Ausdehnungskoeffizient von $\alpha=14,7^{*} 10^{-6} \mathrm{~K}^{-1}$, der nur wenig vom Ursprungsmaterial abweicht.
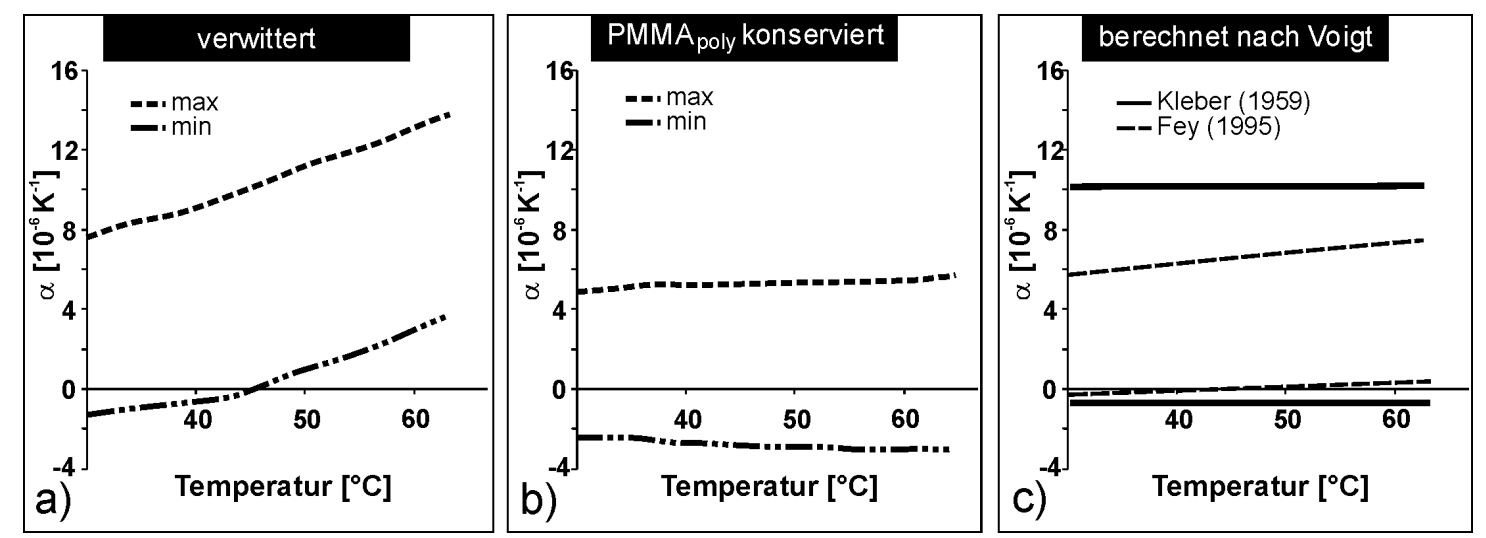

Abb. 7.4: Änderung der Ausdehnungskoeffizienten bei thermischer Beanspruchung auf $65^{\circ} \mathrm{C}$ des Prieborner Marmors: a) verwitterter und b) mittels PMMA poly $_{\text {konservierter Zustand sowie c) }}$ aus der Textur errechnet nach VOIGT (1928) für Ausdehnungskoeffizienten von KLEBER (1959) und FEI (1995).

Da die Ausdehnungskoeffizienten aber deutlich herabgesetzt sind, scheint der hohe $\alpha$-Wert des PMMAs keinen direkten Einfluss auf die Ausdehnung zu besitzen. Aufgrund der häufigen Mikrorisse innerhalb eines verwitterten Marmors ist davon auszugehen, dass eine bestimmte 
Pufferung auftritt (vgl. Kap. 5.1.3), die zum einen die Wirksamkeit der Kontraktion des Calcit-Einkristalls parallel zu den a-Achsen mindert und zum anderen in einem erhöhten Einfluss der starken Dehnung parallel zur c-Achse auf das Gesamtgestein resultiert. Die Volltränkung eines geschädigten Marmors führt zu einer verbesserten Verklebung der Körner (hohe Kohäsion), womit die Pufferung der Mikrorisse reduziert wird (s. Abb. 7.5). Dies resultiert in einer verbesserten Übertragung der Einkristalleigenschaften auf das Verhalten des Gesamtgesteins.

Zusätzlich zu den experimentell ermittelten Ausdehnungskoeffizienten sind die nach den Theorien von VoIGT (1928) aus der Textur berechneten in Abb. 7.4c dargestellt. Hierbei ist eine gute Übereinstimmung der experimentellen Daten der PMMA poly konservierten Probe in der Richtung maximaler Dehnung, mit den errechneten Ausdehnungskoeffizienten der von FEI (1995) für den Einkristall angegebenen, festzustellen. Unklar ist jedoch, weshalb der Marmor parallel zur Richtung minimaler Längenänderung gegenüber den errechneten Werten deutlich stärker kontrahiert. Es ist davon auszugehen, dass die Dehnung des Acrylharzvollgetränkten Marmors annähernd dem eines rissfreien entspricht. Jedoch sollten hier Effekte wie Elastizität oder von der Art und der Geschwindigkeit der Beanspruchung abhängige Festigkeiten (Zug-, Druckfestigkeit) einen deutlichen Einfluss ausüben und je nach experimenteller Durchführung (z.B. Heizrate) zu variierenden Ergebnissen führen.

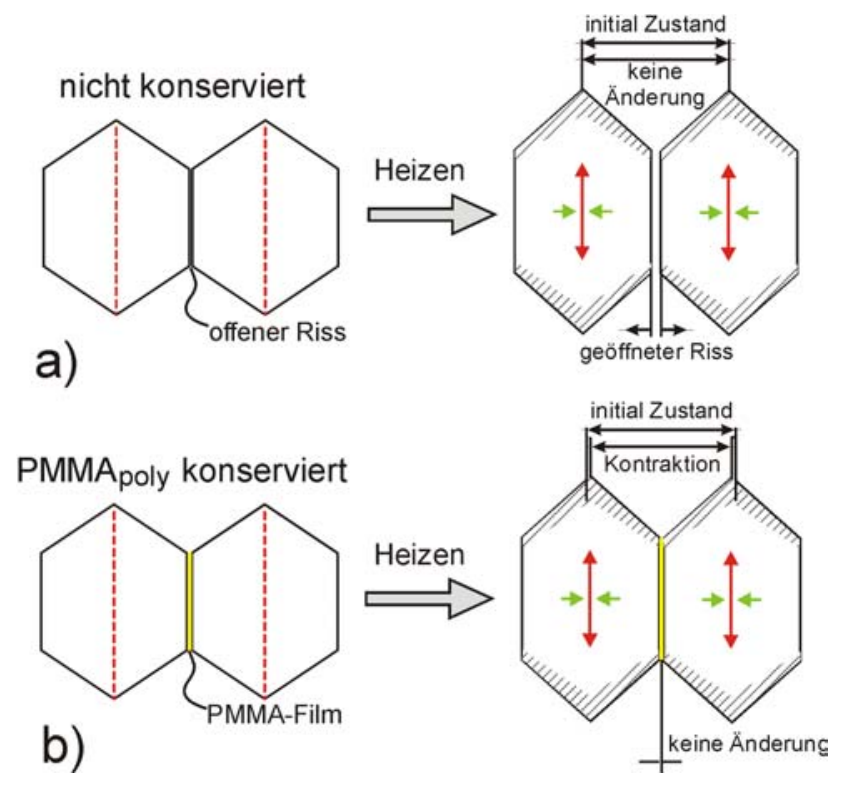

Abb. 7.5: Schematische Darstellung der Aufhebung des Pufferungseffekts der Mikrorisse für die Dilatation von Marmoren durch die Acrylharzvolltränkung: a) nicht konservierter Zustand und b) mit PMMA poly konservierter Marmor.

\section{2 Änderung der Ultraschallgeschwindigkeiten}

Um die Änderung der Ultraschallcharakteristik für thermische Beanspruchung konservierter Marmore zu ermitteln, wurden imprägnierte Gesteinskugeln Schädigungszyklen von $65^{\circ} \mathrm{C}$ und $95{ }^{\circ} \mathrm{C}$ ausgesetzt. Dabei sind für beide Acrylharz-Konservierungen nur geringe Reduktion der Geschwindigkeiten festzustellen. Hingegen zeigen die mit Kieselsäureester imprägnierten 
Proben eine deutliche Absenkung der Ultraschallgeschwindigkeiten. Die gewonnen Daten sind in Abb. 7.6 exemplarisch für den Prieborner Marmor dargestellt.

Für die $\underline{\text { PMMA }}_{\text {sol }}$ konservierte Probe aus Prieborner Marmor ist während des ersten Heizzyklus auf $65^{\circ} \mathrm{C}$ parallel zur X-Richtung (maximale Richtung) eine $\mathrm{V}_{\mathrm{p}}$-Änderung von $\mathrm{V}_{\mathrm{p}_{\_} 20}=4,54 \mathrm{~km} / \mathrm{s}$ auf $\mathrm{V}_{\mathrm{p}_{-} 65}=4,11 \mathrm{~km} / \mathrm{s}$ zu ermitteln, womit $\Delta \mathrm{V}_{\mathrm{p}_{-} 20-65}=0,43 \mathrm{~km} / \mathrm{s}$ beträgt. Für den zweiten Heizzyklus auf $90{ }^{\circ} \mathrm{C}$ ist eine Herabsetzung in der gleichen Richtung auf $\mathrm{V}_{\mathrm{p} 90}=3,97 \mathrm{~km} / \mathrm{s}$ auszumachen. Damit ist die Differenz nur noch bei Werten $\Delta \mathrm{V}_{\mathrm{p}_{-} 65 \text { - }}$ ${ }_{90}=0,14 \mathrm{~km} / \mathrm{s}$. Die weiteren Gefügerichtungen verhalten sich dabei vergleichbar (Abb. 7.6a,b).

\section{$\mathrm{PMMA}_{\text {sol }}$ konserviert}

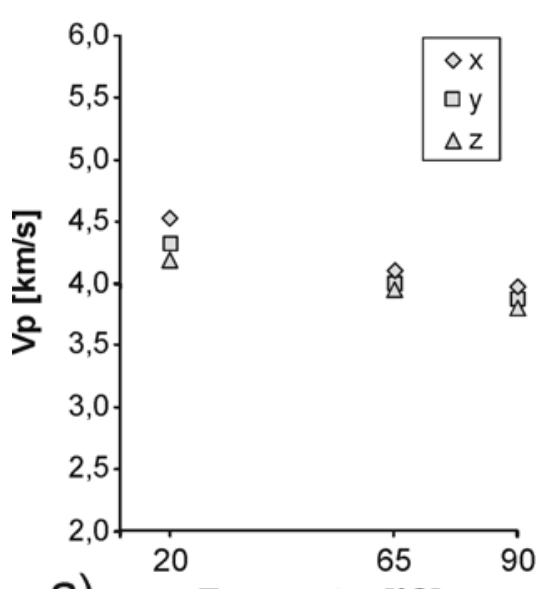

a)
PKSE konserviert

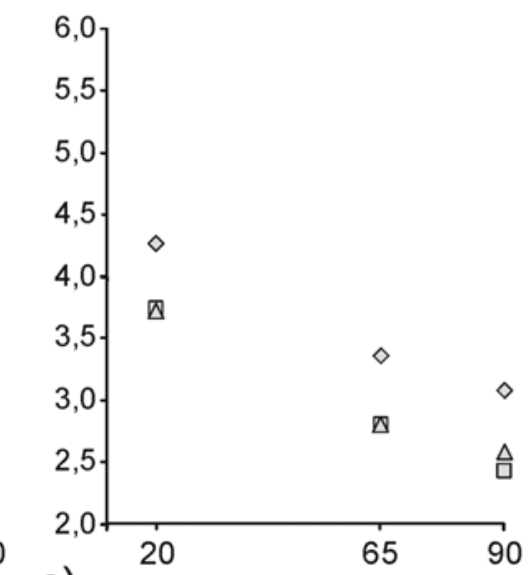

C) Temperatur $\left[{ }^{\circ} \mathrm{C}\right]$

\section{PMMA poly konserviert}

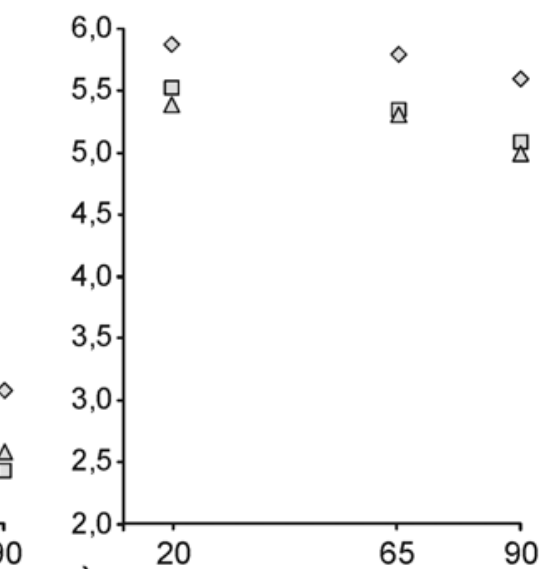

e) Temperatur $\left[{ }^{\circ} \mathrm{C}\right]$

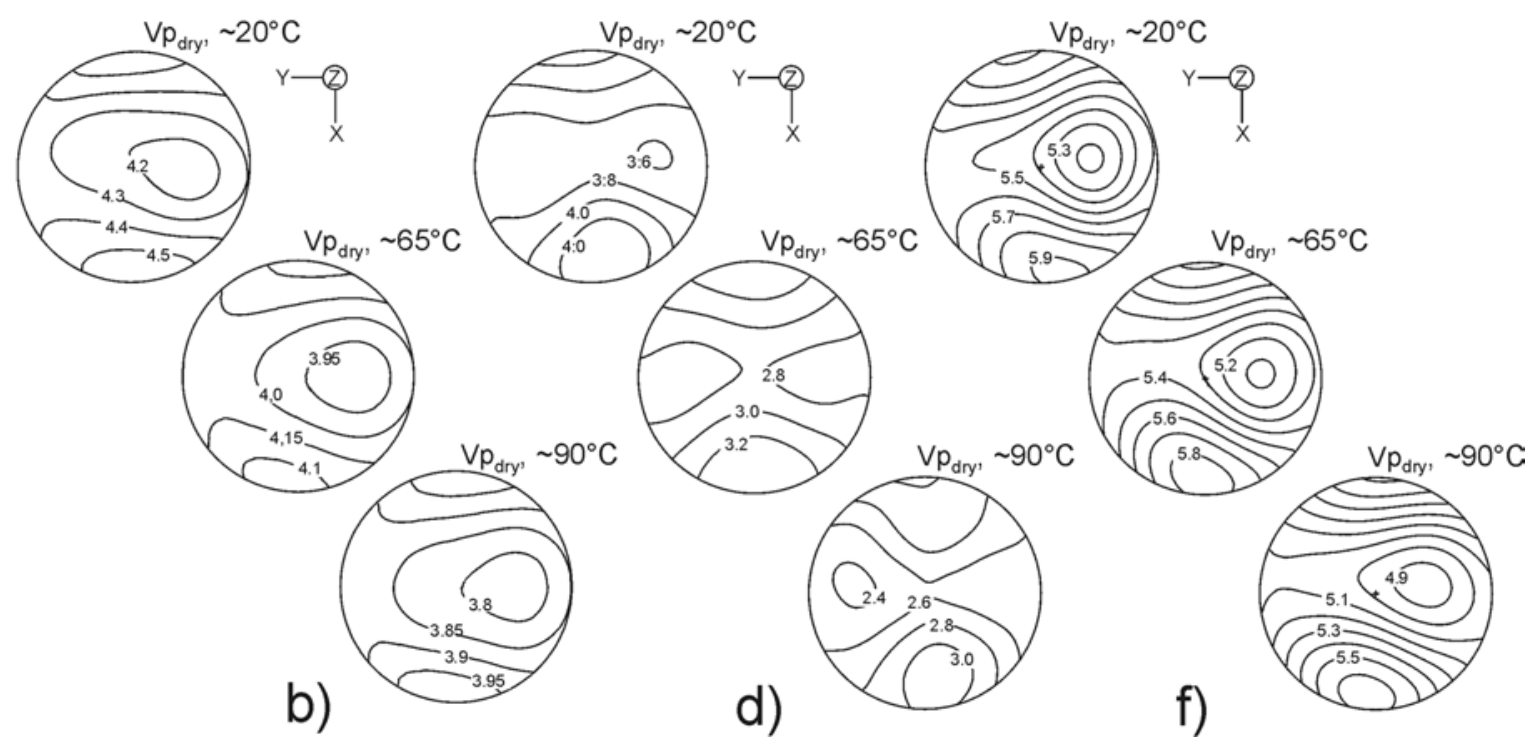

Abb. 7.6: Herabsetzung der Kompressionswellengeschwindigkeiten konservierter Marmore bei thermischer Beanspruchung auf $65{ }^{\circ} \mathrm{C}$ und $90{ }^{\circ} \mathrm{C}\left(20^{\circ} \mathrm{C}=\right.$ Ausgangszustand): a,c,e): $\mathrm{V}_{\mathrm{p}^{-}}$ Herabsetzung gegen die Beanspruchungstemperatur und $b, d, f) V_{p}$-Verteilungen für die entsprechenden Temperaturen (Schmidt'sches Netz, untere Halbkugel).

Damit ist eine deutliche Herabsetzung der Ultraschallgeschwindigkeiten bei Erwärmung oberhalb der Glasübergangstemperatur des PMMA ${ }_{\text {sol }}\left(\mathrm{T}_{\mathrm{g}}\right.$ bei ca. $\left.60^{\circ} \mathrm{C}\right)$ festzustellen und eine kaum messbare Reduktion bei höheren Temperaturen. Damit stimmen die $\mathrm{V}_{\mathrm{p}}$ - $\mathrm{A}$ nderungen 
auch mit den experimentellen Dilatometerdaten der thermischen Längenänderungen überein. So ist eine erhebliche Restdehnung für den Übergang der $\mathrm{T}_{\mathrm{g}}$ bei Erwärmung der Probe auf $65^{\circ} \mathrm{C}$ festzustellen, wohingegen der Heizzyklus auf $90{ }^{\circ} \mathrm{C}$ nur zu geringen permanenten Längenänderungen führt (vgl. Tab. 7.1). Die Gesamtabnahme ist mit $\Delta \mathrm{V}_{\mathrm{p}_{-} \text {kons-90 }}=0,57 \mathrm{~km} / \mathrm{s}$ nur sehr gering und wird vom $\mathrm{T}_{\mathrm{g}}$ bedingt. Damit kann die Herabsetzung weitgehend mit einer Erweichung des Acrylharzes und weniger mit einer Rissneubildung begründet werden.

Die PKSE imprägnierte Probe aus Prieborner Marmor zeigt für beide thermische Beanspruchungszyklen eine deutliche Absenkung der Ultraschallgeschwindigkeiten. Während des ersten Heizzyklus wird $V_{p}$ parallel zur X-Richtung von $V_{p_{-} 20}=4,26 \mathrm{~km} / \mathrm{s}$ auf $\mathrm{V}_{\mathrm{p}_{-} 65}=3,36 \mathrm{~km} / \mathrm{s}$ stark reduziert. Der zweite Heizzyklus von $65{ }^{\circ} \mathrm{C}$ auf $90{ }^{\circ} \mathrm{C}$ bewirkt eine Reduktion auf $\mathrm{V}_{\mathrm{p}_{-} 90}=3,07 \mathrm{~km} / \mathrm{s}$. Damit liegt die Differenz bei $\Delta \mathrm{V}_{\mathrm{p}_{-} 65-90}=0,29 \mathrm{~km} / \mathrm{s}$ (s. Abb. 7.6c,d).

Die Gesamtherabsetzung von $\Delta \mathrm{V}_{\mathrm{p}_{-} \text {kons- } 90}=1,19 \mathrm{~km} / \mathrm{s}$ fällt folglich im Vergleich zu den anderen Konservierungsmethoden wesentlich höher aus. Dieses Verhalten ist auf die nur geringe Anhaftung des PKSE auf Kristalloberflächen zurückzuführen. Da der Kieselsäuereester die einzelnen Kristalle nicht miteinander verklebt, können die anisotropen Dehnungseigenschaften bei thermischer Erwärmung wieder frei wirken und die Risse weiten.

Die mittels $\underline{P M M A}_{\text {poly }}$ imprägnierte Probe aus Prieborner Marmor zeigt beim Heizen auf $65^{\circ} \mathrm{C}$ für Maximum $\mathrm{V}_{\mathrm{p}}$ parallel zur X-Richtung eine Herabsetzung von $\mathrm{V}_{\mathrm{p}_{\_} \text {kons }}=5,87 \mathrm{~km} / \mathrm{s}$ auf $\mathrm{V}_{\mathrm{p}_{-} 45}=5,79 \mathrm{~km} / \mathrm{s}$ und parallel zur Z-Richtung (Minimum $\mathrm{V}_{\mathrm{p}}$ ) eine Reduktion von $\mathrm{V}_{\mathrm{p}_{-} \text {kons }}=5,37 \mathrm{~km} / \mathrm{s}$ auf $\mathrm{V}_{\mathrm{p}_{-} 45}=5,29 \mathrm{~km} / \mathrm{s}$. Damit liegen in beiden Richtungen die Geschwindigkeitsherabsetzungen für den Heizzyklus auf $65^{\circ} \mathrm{C}$ unter $0,1 \mathrm{~km} / \mathrm{s}$ und damit unter der Auflösungsgrenze der Messmethode. Für den zweiten Heizzyklus auf $90{ }^{\circ} \mathrm{C}$ ist eine deutlichere Abnahme festzustellen. Hierbei wird die X-Richtung von $\mathrm{V}_{\mathrm{p}_{-} 45}=5,79 \mathrm{~km} / \mathrm{s}$ auf $\mathrm{V}_{\mathrm{p}_{-} 90}=5,59 \mathrm{~km} / \mathrm{s}$ und die Z-Richtung von $\mathrm{V}_{\mathrm{p}_{-} 45}=5,29 \mathrm{~km} / \mathrm{s}$ auf $\mathrm{V}_{\mathrm{p}_{-} 90}=4,97 \mathrm{~km} / \mathrm{s}$ reduziert (s. Abb. 7.6e,f).

Wie auch für die $\mathrm{PMMA}_{\text {sol }}$ konservierten Proben, weisen die $\mathrm{PMMA}_{\text {poly }}$ imprägnierten damit eine deutlichere Herabsetzung der Ultraschallgeschwindigkeiten im Bereich des Durchschreitens der Glasübergangstemperatur des Kunststoffes auf ( $\mathrm{T}_{\mathrm{g}}$ bei ca. $\left.85^{\circ} \mathrm{C}\right)$. Daher kann auch hier festgestellt werden, dass es sich um eine weitgehende Erweichung des Acrylharzes handelt und weniger um eine neue Rissbildung. 


\section{8 Überführung der Laborerkenntnisse ans Bauwerk}

Aus wissenschaftlicher Sicht stellt sich die Frage, welche Bedeutung die im Labor gewonnenen Erkenntnisse des Materialverhaltens von Marmoren direkt für die vor Ort Problematik besitzen. Um dies $\mathrm{zu}$ überprüfen sollen im Folgenden, anhand einzelner Fallbeispiele, ausgewählte Fragestellungen erörtert werden. Als Modellfall für die Verwitterungsproblematik von Marmoren dient das Marmorpalais im Neuen Garten in Potsdam. Das Gebäude ist aufgrund der Verwendung unterschiedlicher Marmortypen im Außenbereich und seiner langen und wechselhaften Geschichte besonders geeignet.

Im Folgenden sollen zunächst einige Hintergrundinformationen über das Gebäude und die bauhistorische Entwicklung gegeben werden. Hierbei stehen Informationen, über die Verwendung der Materialien, die durchgeführte Restaurierungsmaßnahmen sowie die klimatischen Bedingungen im Vordergrund. Ein wesentlicher Punkt ist die Frage, inwieweit die makroskopisch erkennbaren Schadensphänomene auf den inneren Aufbau des Marmors zurückzuführen sind und ob eine materialspezifische Verwitterung stattgefunden hat.

Eine wesentliche Fragestellung für die Entwicklung von Sanierungsstrategien geschädigter Marmore, ist die diagnostische Erfassung, vor allem auch des Erhaltungszustandes des unzugänglichen Inneren, von Marmorbauteilen. Der Einfluss des Gefüges und der daraus resultierenden Richtungsabhängigkeit von Ultraschallgeschwindigkeiten soll anhand von Ultraschall-tomographischen Untersuchungen diskutiert werden. Dazu wurden detaillierte Gefügekartierungen in Verbindung mit Ultraschalluntersuchungen exemplarisch an Säulen aus Kauffunger und Prieborner Marmor durchgeführt.

In einem weiteren Fallbeispiel sollen die Möglichkeiten einer Konservierung von Marmoren betrachtet werden. Da viele Objekte nicht oder nur unter großem Aufwand aus Gebäuden entnommen werden können, stellt sich die Frage, ob eine Volltränkung auch vor Ort durchgeführt werden kann. Dies soll anhand einer im Labor nachgestellten Volltränkung eines Gesteinzylinders unter Berücksichtigung der vor Ort Situation überprüft werden.

\subsection{Das Marmorpalais im Neuen Garten Potsdam}

Das Marmorpalais gehört zum Ensemble der preußischen Schlösser und Gärten, die von der gleichnamigen Stiftung Berlin Brandenburg verwaltet wird. Das Gebäude befindet sich im Neuen Garten von Potsdam am westlichen Ufer des Heiligen Sees. Das Marmorpalais (s. Abb. 8.1) gehört zu den bedeutsamen Kulturgütern, die 1990 von der UNESCO zum Weltkulturerbe erklärt wurden.

Das Gebäude wurde nach Plänen von Karl v. Gontard für Friedrich Wilhelm II. zwischen 1787 - 1791 errichtet. Die Architektur des würfelförmigen, zweigeschossigen Hauptbaus mit Belvedere orientiert sich an palladinischen Vorbildern. Der für Säulen, Friese und Gesimse sowie zum Innenausbau verwendete Marmor wird in Akten und Schreiben aus der Erbauungszeit als schlesischer Marmor angesprochen und stammt aus den polnischen Ortschaften Kauffungen (Wojcieszów), Grosskunzendorf (Slawniowice) und Prieborn 
(Przeworno). Zusätzlich wurde aber auch Carrara Marmor (Italien) verbaut, so dass ursprünglich vier verschiedene Vertreter dieses Natursteins verwendet wurden.
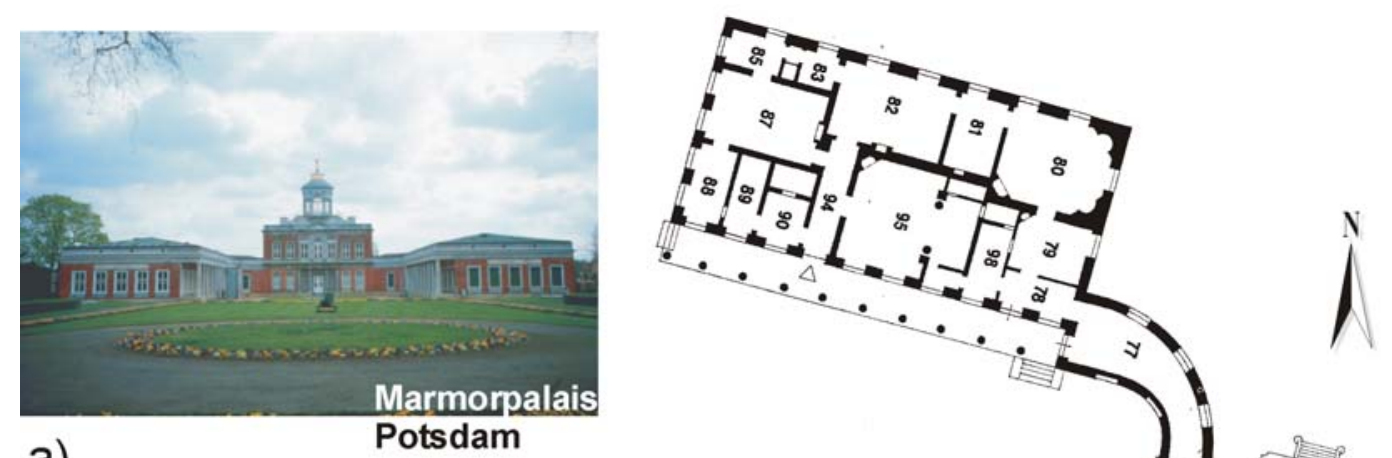

a)

Potsdam
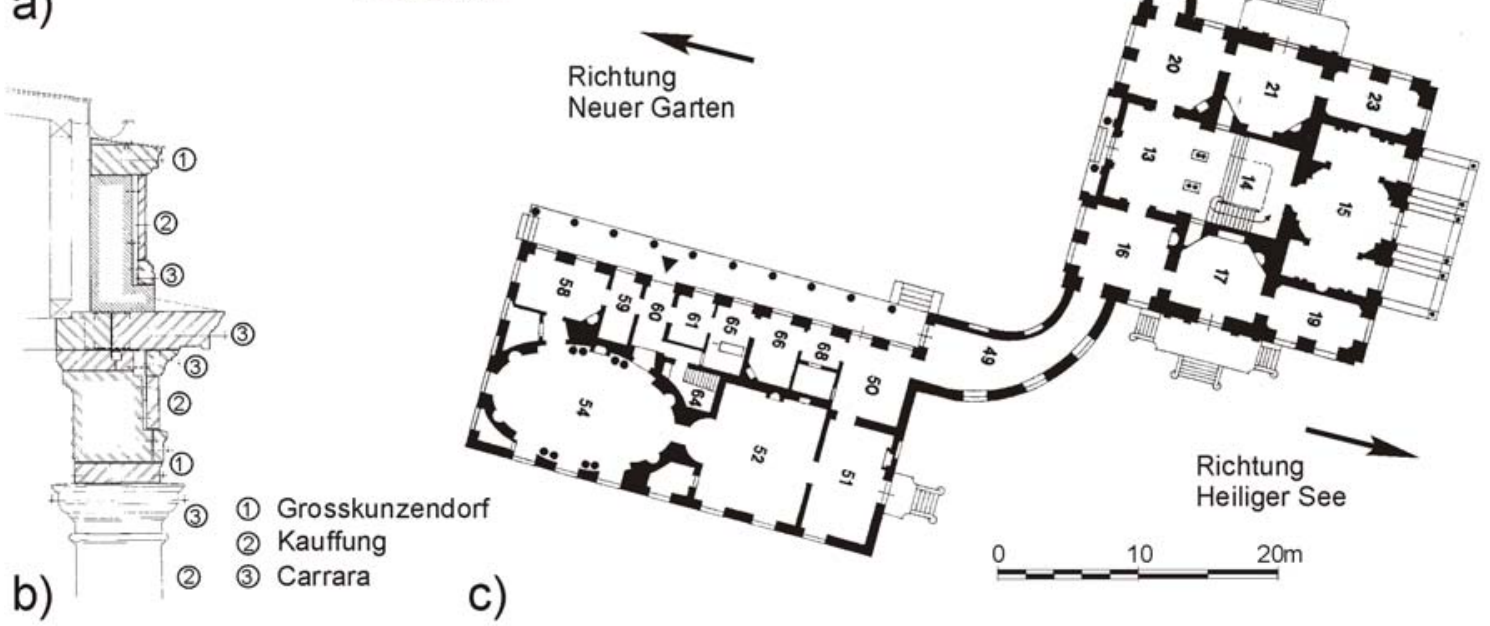

Abb. 8.1: Marmorpalais im Neuen Garten Potsdam: a) Frontansicht in Richtung Heiliger See, b) Architrav und Attika der Hofseite im Anschnitt und c) Grundriss mit eingetragenen Räumlichkeiten des Erdgeschosses.

Als schon wenige Jahre nach Errichtung des Hauptbaus die Räumlichkeiten zu eng geworden waren, wurde das ursprüngliche Gebäude um zwei eingeschossige Seitenflügel erweitert. Diese wurden im Jahre 1797 nach Entwürfen von Carl Gotthard Langhans begonnen und erst 1843 - 1845 von Ludwig Persius und Ludwig Ferdinand Hesse vollendet.

Da zu Beginn der Bauzeit insbesondere der Kauffunger Marmor nicht ausreichend schnell besorgt werden konnte, kam es zu Engpässen des Materialnachschubs. Als Lösung erwies sich die Verwendung von Spolien der ehemaligen Rehgarten Kolonnade im Park Sanssouci, die ebenfalls aus Kauffunger Marmor aufgebaut war. Die von Georg Wenzeslaus von Knobelsdorff entworfene Rehgartenkolonnade, wies zwar nach wenigen Jahrzehnten Standzeit statische Probleme auf, eignete sich jedoch zur Weiterverwendung. Das Material wurde für alle Säulen, für Architrav, Attika und Hauptgesims im Innenhof der Seitenflügel umgearbeitet. Dazu wurden z.B. für die Friese die Spolien in ca. $10 \mathrm{~cm}$ breite Platten aufgesägt.

Das als Sommerschloss konzipierte Bauwerk diente nur selten als Wohnresidenz. Ab 1932 wurde das Gebäude als Schlossmuseum genutzt. Im zweiten Weltkrieg erlitt das Gebäude mehrere Brandschäden, wobei Ziegelmauerwerk und Dachabdeckung besonders betroffen 
waren. Nach 1945 richtete die Rote Armee im Marmorpalais ein Offizierscasino ein. In der ehemaligen DDR diente das Gebäude bis in die 80er Jahre als Militärmuseum.

Der Grossteil des Gebäudes und damit auch der verwendeten Marmore ist seit über 200 Jahren der Bewitterung ausgesetzt. Die in ca. $30 \mathrm{~m}$ über NN liegende Stadt Potsdam in Brandenburg gilt als klimatisch gemäßigt humid. Die Jahresniederschlagsmenge von im Mittel ca. $600 \mathrm{~mm}$ ist auf rund 180 Regentage verteilt. Die in Abb. 8.2 dargestellte Temperatur- und Luftfeuchtigkeitsentwicklung ist für den Jahresverlauf aus Messungen zwischen 1893 bis 2002 von der Säkulärstation in Potsdam (Telegrafenberg) aufgenommen worden.
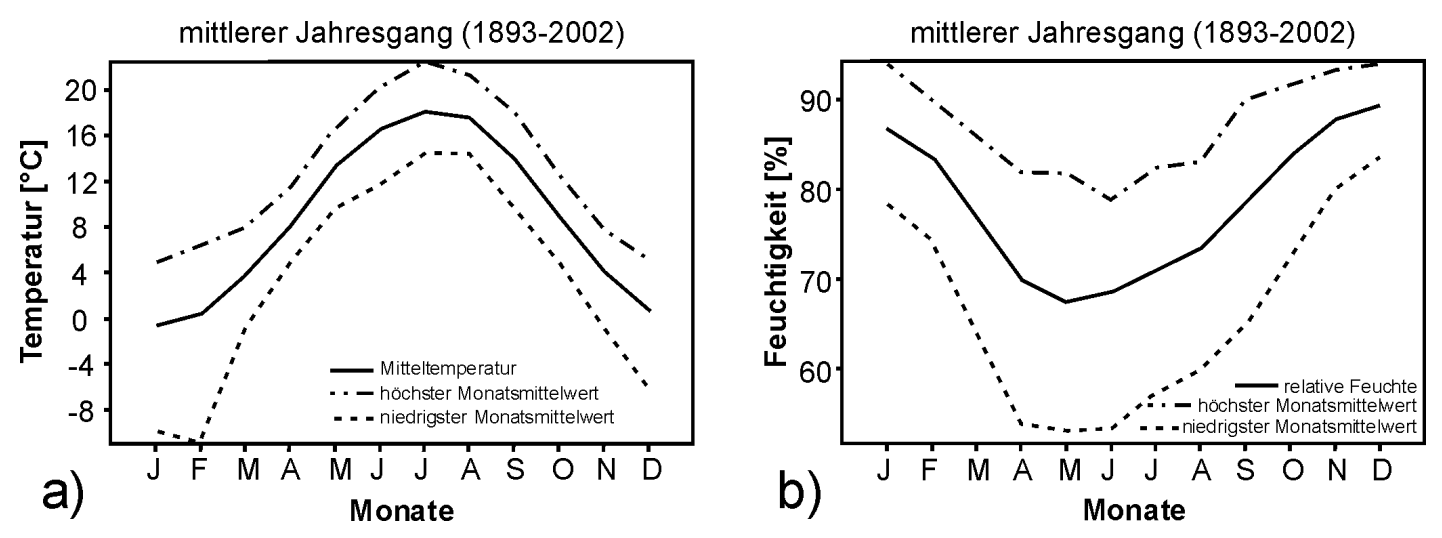

Abb. 8.2: Mittlere Werte a) der Temperatur und b) der relativen Luftfeuchtigkeit im Jahresverlauf in Potsdam (Daten von der Säkulärstation Potsdam, Telegrafenberg).

Die ersten grundlegenden Restaurierungsmaßnahmen begannen schon vor der politischen Wende in der DDR. Dabei kam es zum Austausch von Marmoren, wobei aus finanziellen Gründen sowie der begrenzten Verfügbarkeit der schlesischen Marmore auf den Ersatz mit Originalmaterial verzichtet werden musste. Der Carrara Marmor wurde durch äußerlich ähnlich erscheinenden dolomitischen Sivec Marmor ausgetauscht. Als Ersatz des grobkörnigen Grosskunzendorfer Marmors für Attika und Architrav diente die kubanische Sorte Grissibonay und der Kauffunger Marmor wurde durch den Miokazi Marmor ersetzt. Nach der Wiedervereinigung wurden die Restaurierungsarbeiten intensiviert, womit es auch wieder zum Einsatz von vergleichbaren Originalwerksteinen kam. Eine Ausnahme stellt der nicht mehr zur Verfügung stehende Prieborner Marmor dar, welcher durch den tschechischen Lindewiese Marmor ersetzt wurde. Die Restaurierungsarbeiten dauern bislang noch an. Hierbei steht insbesondere die Sanierung von Marmorsäulen der Seitenflügel und des zum Heiligen See zugewandten Balkons zur Zeit noch aus. Die Säulen bestehen aus Kauffunger und Prieborner Marmor.

\subsubsection{Gefügebedingte Schadensphänomene}

Die noch vorhandenen originalen Marmorbauteile des Marmorpalais zeigen teilweise erhebliche Schäden. Hierbei handelt es sich zumeist um charakteristische Entfestigungsphänomene, welche signifikante Abhängigkeiten vom Gesteinsgefüge aufweisen. Die wesentlichen Schädigungsmerkmale können den jeweiligen in Kap 4.2.1 angeführten Korngefügetypen zugeordnet werden. 
Für den Carrara und den Prieborner Marmor (Korngefügetyp I) ist das Hauptschadensbild eine ausgeprägte Verwitterung der Gesteinsoberflächen. Diese ist auf eine Entfestigung an Korngrenzen zurückzuführen, was sich im fortgeschrittenen Verwitterungszustand durch sandiges Abzuckern einzelner Partikel äußert. Resultierend weisen diese Marmore einen relativ starken Materialverlust auf und bilden ein raues Oberflächenrelief aus. Die ursächlichen Verwitterungsprozesse sind sicherlich die initiale thermische Gefügeauflockerung sowie eine starke Einwirkung chemischer Lösungsprozesse in oberflächennahen Bereichen. Das Phänomen des Herauslösens einzelner Körner ist hier auf eine gesteinsdurchdringende Rissentfestigung an Korngrenzen zurückzuführen.

Bezüglich der Oberflächenrückwitterung weisen beide Marmore in lokalen Bereichen Unterschiede in der Intensität auf, was durch die variierende mineralogische Zusammensetzung hervorgerufen wird. Dabei sind die dunklen Lagen des Prieborner Marmors gegenüber den hellen deutlich verwitterungssensibler. Für den Carrara Marmor sind zumeist erhebliche Unterschiede in der Verwitterungsrate zwischen den schlierenartigen dunklen Bändern und der hellen Matrix des Gesteins zu beobachten. Die dunklen Lagen, welche von feinstkörnigen Verwachsungen von Dolomit und Calcit gebildet werden, sind dabei durch ein positives Relief gekennzeichnet und somit verwitterungsresistenter.

Für den Prieborner Marmor können weiterhin materialcharakteristische Makrorisse als häufig zu beobachtendes Phänomen festgestellt werden. Hierbei können sowohl herausgewitterte (positives Relief) als auch tiefgründig verwitterte offene Risse (negatives Relief) beobachtet werden (s. Abb. 8.3a,b). Die Makrorisse folgen dabei gesteinsinternen Vorzeichnungen in Form von mineralisierten bzw. versiegelten Risssystemen. Das unterschiedliche Verwitterungsverhalten ist vorwiegend auf Variationen in der mineralogischen Zusammensetzung zurückzuführen. Die hervorstehenden Risse sind dabei mit Quarz ausgefüllt.

Das charakteristische Verwitterungsmerkmal des Grosskunzendorfer Marmors (Korngefügetyp II) sind parallel verlaufende offene Risssysteme (Abb. 8.4a). Diese haben eine relativ deutliche Öffnungsweite im mm-Bereich. Im Gegensatz zu den Makrorissen des Prieborner Marmors sind hier keine Gefügevorzeichnungen makroskopisch sichtbar. Der Verlauf der Risse lässt jedoch vermuten, dass sie auf Vorzeichnungen im mikroskopischen Maßstab zurückzuführen sind. Ein weiteres Verwitterungsmerkmal des Grosskunzendorfer Marmors ist die Rückwitterung der Oberfläche, die im Wesentlichen auf chemische Lösungsprozesse zurückgeführt werden kann. Hierbei ist eine deutliche Reliefbildung entlang von Korngrenzen und im Gegensatz zum Prieborner Marmor auch an kristallinternen Diskontinuitäten feststellbar. Nur an einzelnen, zumeist besonders exponierten Bauteilen (z.B. Balustern), kann auch ein sandiges Abzuckern der Oberflächen und damit eine weit vorangeschrittene Gefügeentfestigung festgestellt werden (Abb. 8.4b). Dieses Verwitterungsphänomen ist vor allem an Bauteilkanten $\mathrm{zu}$ beobachten und kann vermutlich auf eine frühe besonders intensive Gefügebeanspruchung durch die Steinmetzbearbeitung zurückgeführt werden.

Der Kauffunger Marmor (Korngefügetyp III) unterscheidet sich bezüglich seiner Verwitterungsphänomene deutlich von den anderen am Marmorpalais verbauten Marmore. Die Rückwitterung der Gesteinsoberfläche erscheint im Vergleich geringer. Ebenfalls kann 
kein sandiges Ablösen der Oberfläche beobachtet werden, was darauf schließen lässt, dass die Entfestigung des Kornverbandes wesentlich geringer als bei den anderen Marmoren vorangeschritten ist. Die eigentliche Verwitterung erfolgt vielmehr an präexistierenden mineralisierten bzw. versiegelten Risssystemen. Hierbei scheinen einige der Risse geöffnet zu sein, wo hingegen an anderen lediglich eine deutliche Rückwitterung zu erkennen ist (Abb. 8.3c,d). Insbesondere zwischen den geöffneten Makrorissen können gehäuft Materialausbrüche beobachtet werden.
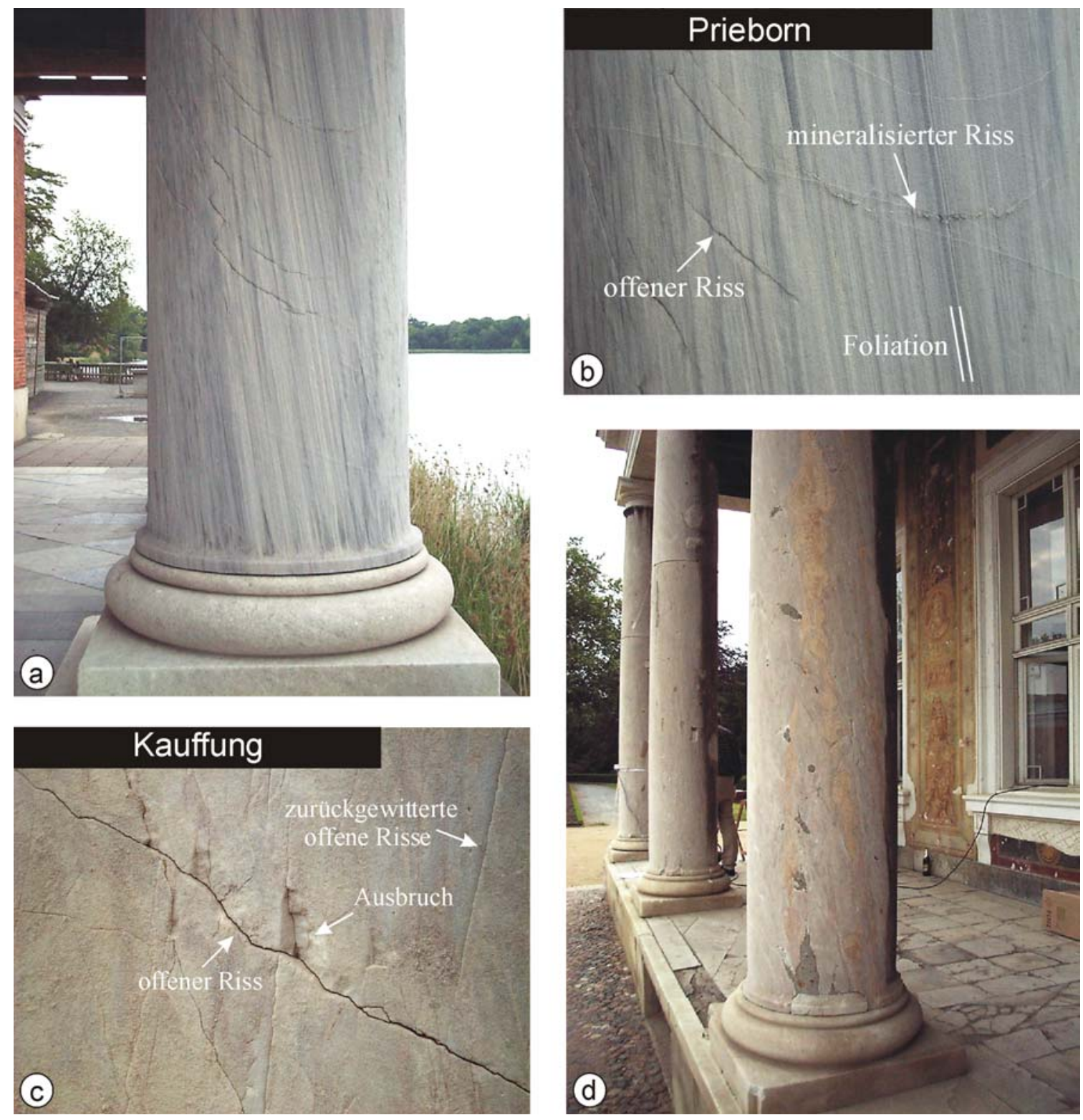

Abb. 8.3: a) Unterer Teil einer Säule aus Prieborner Marmor mit unterlagerndem Säulenprofil aus Grosskunzendorfer Marmor als statische Bauelemente des zum See ausgerichteten Balkons (Säulendurchmesser $55 \mathrm{~cm}$ ). b) Detailansicht der Prieborner Marmorsäule zeigt eine deutliche Reliefbildung durch heterogene Rückwitterung des Lagenbaus und verschiedene offene und hervortretende mineralisierte Risse. c) Detailansicht einer Kauffunger Marmorsäule mit verschiedenen Rissen und Ausbrüchen. d) Kauffunger Marmorsäule des nördlichen Seitenflügels mit gelblichen, das Bauteil senkrecht durchziehenden Dolomitadern und zahlreiche mittels Mörtel verschlossene Ausbrüche (Säulendurchmesser $55 \mathrm{~cm}$ ). 

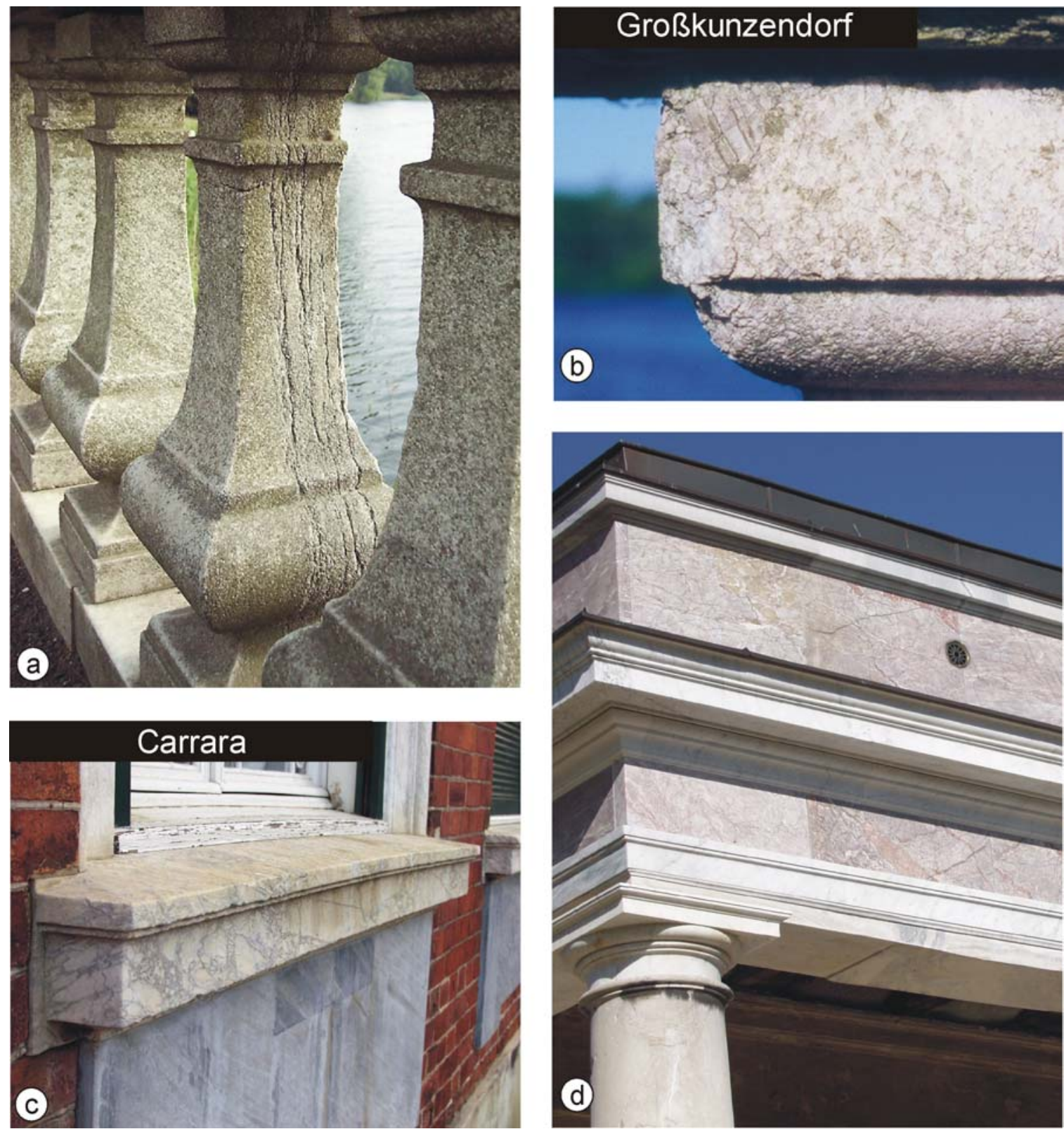

Abb. 8.4: a) Balustrade aus Grosskunzendorfer Marmor (Seeseite) mit zum Teil extremer Verwitterung durch parallel verlaufende Risssysteme. b) Detailansicht eines Baluster mit Substanzverlust an den Bauteilkanten durch Ausbrechen von Bruchstücken. c) Fensterbank aus Carrara Marmor mit starker Reliefbildung aufgrund deutlicher Rückwitterung der hellen Gesteinsbereiche. d) Architrav mit darüber liegender Attika, gegliedert durch Verwendung verschiedener Marmorsorten (vgl. Abb. 8.1b).

\subsection{Fallstudie: Ultraschalltomographie an Marmorsäulen}

Die Ultraschallanalytik zur Ermittlung des Erhaltungszustandes von Naturwerksteinen hat in den letzten Jahren, vor allem in der Denkmalpflege aufgrund der zerstörungsfreien Durchführbarkeit, zunehmend an Bedeutung gewonnen. Dabei ermöglicht die tomographische Anwendung der Ultraschallanalytik eine ortsauflösende Zustandscharakterisierung des unzugänglichen Inneren eines Objektes. Die Ultraschalltomographie kann helfen unterschiedlich entfestigte Natursteinbereiche zu detektieren, um Sanierungskonzepte optimal auf das Objekt anzupassen. 
Zur Rückkopplung der im Labor gewonnen Erkenntnisse der Ultraschallcharakteristik bestimmter Marmore mit den vor Ort Verhältnissen wurden an zwei ausgewählten Säulen des Marmorpalais in Potsdam aus Prieborner und Kauffunger Marmor ultraschalltomographische Untersuchungen durchgeführt. Um zu überprüfen inwieweit die makroskopischen Schäden mit den Ultraschalldaten übereinstimmen, erfolgte zusätzlich die Aufnahme des makroskopischen Gefügeinventars sowie der Schadensphänomene mittels detaillierter Säulenkartierungen. Die Ultraschalluntersuchungen am Gebäude wurden in Zusammenarbeit mit der Abteilung Geophysik der Technischen Universität in Freiberg durchgeführt.

\subsubsection{Ultraschalltomographische Untersuchungen}

Der Begriff Tomographie kommt aus dem griechischen und bedeutet soviel wie Schnittbild. Er umfasst die Rekonstruktion eines Schnittes durch einen Körper durch Messungen außerhalb des Objektes. Die mathematische Basis für die Tomographie wurde von RADON (1917) eingeführt. Er zeigte, dass ein Objekt durch ein komplettes Set seiner Projektionen exakt rekonstruiert werden kann. Mit der Weiterentwicklung der digitalen Datenverarbeitung fand die Methode, zuerst in der Astronomie, folgend mit großem Erfolg in der Medizin und mehr aktuell in der Geophysik (z.B. NOLET, 1987), praktische Anwendung. Bezüglich der Durchführung im Bereich der Denkmalpflege befindet sich eine ausführliche Beschreibung des Verfahrens z.B. in LiNDNER et al. (1999) und zur Anwendung an Bauteilen aus Marmor in SIMON (2001).

Für die Ultraschalluntersuchungen der Säulen des Marmorpalais wurden sowohl Kompressionswellen (46 kHz, magnetostriktiver Geber) als auch Scherwellen Messungen (500 kHz, piezoelektrischer Geber) durchgeführt. Bezüglich der Strahlengeometrie wurde eine Senderposition mit sieben Empfängerpositionen auf einer Ebene um die Säule kombiniert, woraus die Erfassung eines Sektors von $67^{\circ}$ während jedes Messzyklus resultiert (s. Abb. 8.5a). Durch achtfaches Drehen dieser Anordnung konnte eine weitgehend vollständige Abdeckung der Säulenquerschnitte erreicht werden (Abb. 8.5b).
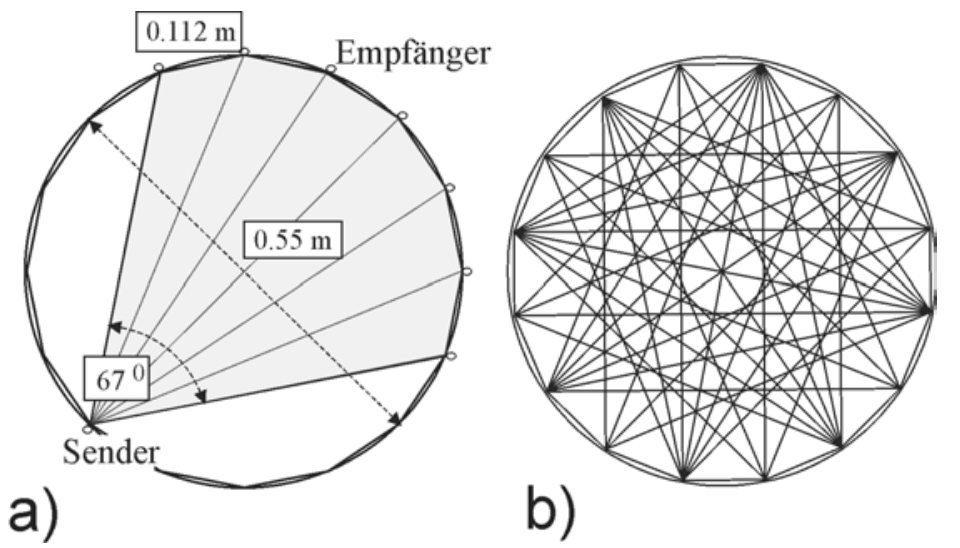

Abb. 8.5: Schematische Darstellung der für die Tomographie verwendeten Sender-Empfängeranordnung. Der Durchmesser der Säulen beträgt 0,55 m. a) Einfache Anordnung mit einem Sender und sieben Empfängern und b) vollständige Abdeckung welche durch Drehen der Sender-Empfängeranordnung erreicht wird. 
Die Bestimmung der 2 D-Querschnittsverteilung erfolgte durch eine tomographische Rekonstruktion mittels des Software Programms Migratom (Wellenfront-Migration; s. JACKSON \& TwEETON, 1994). Die Berechnung wurde sowohl mit geraden als auch gekrümmten Strahlenverläufen durchgeführt. Jedoch sind in der vorliegenden Arbeit nur Tomogramme dargestellt deren Rekonstruktion mittels gerader Strahlen erfolgte. Diese Vorgehensweise wurde gewählt, da zum einen keine signifikanten Unterschiede zwischen den Berechnungsverfahren festzustellen sind, zum anderen liegen auch die gemessenen Geschwindigkeitskontraste unterhalb $40 \%$ womit nach TwEETON et al. (1992) die Berechnung mit geraden Strahlenverläufen zu akzeptablen Ergebnissen führen sollte.

\subsubsection{Prieborner Marmor}

\subsubsection{Schadens- und Gefügekartierung}

Die untersuchte Säule aus Prieborner Marmor befindet sich auf der seezugewandten Seite des Marmorpalais und ist in eine Balustrade integriert. Sie trägt mit weiteren fünf Säulen einen Balkon und stellt damit ein konstruktives, d.h. tragendes Bauelement dar. Makroskopisch weist die Säule eine deutliche Schädigung auf, die sich in einer starken Rückwitterung der Oberfläche äußert und deutlich durch eine Reliefbildung der Foliationslagen (dunkel- und hellgraue Bänderung) geprägt ist. Hierbei ist eine höhere Verwitterungssensibilität für die dunklen Lagen des Gesteins festzustellen. Die makroskopischen Gefügeelemente sind in Abb. 8.6 schematisch dargestellt. Die Foliation streicht N-S und zeigt ein steiles Einfallen nach W. Weiteres wesentliches Gefügeelement sind zwei Rissscharen wobei Rissgeneration C1 steil nach E einfällt und Rissgeneration C2 ein mittleres Einfallen nach NW zeigt (vgl. Abb. 8.6).

Gefügekundlich betrachtet wird das Risssystem C1 im Wesentlichen von offenen Rissen dominiert, wobei nicht eindeutig geklärt werden kann wie weit sich diese Öffnung ins Gesteinsinnere fortsetzt. Zweifelsfrei ist an den Rissflanken im Oberflächenbereich eine deutliche Rückwitterung zu erkennen, welche vor allem auf chemische Lösungsprozesse und ein „sandiges Abzuckern“ der Körner zurückgeführt werden kann. Im Gegensatz dazu treten bei der Rissgeneration C2 vorwiegend mineralisierte Risse auf, die aufgrund der Gesamtrückwitterung der Säulenoberfläche ein positives Relief formen (vgl. auch Makrorissschema Abb. 8.12). Es ist davon auszugehen, dass insbesondere die offenen Risse C1 einen hohen Einfluss auf das mechanische Verhalten und auf die Ultraschallgeschwindigkeiten ausüben, da sie Flächen geringer Kohäsion darstellen.

Bezüglich der makroskopischen Gefügeelemente (Ausbildung der Foliation, etc.) ist das Säulenmaterial mit der Laborprobe aus Prieborner Marmor identisch. Damit kann davon ausgegangen werden, dass die untersuchten Materialien auch im Mikrobereich weitgehend vergleichbar ausgebildet sind. 

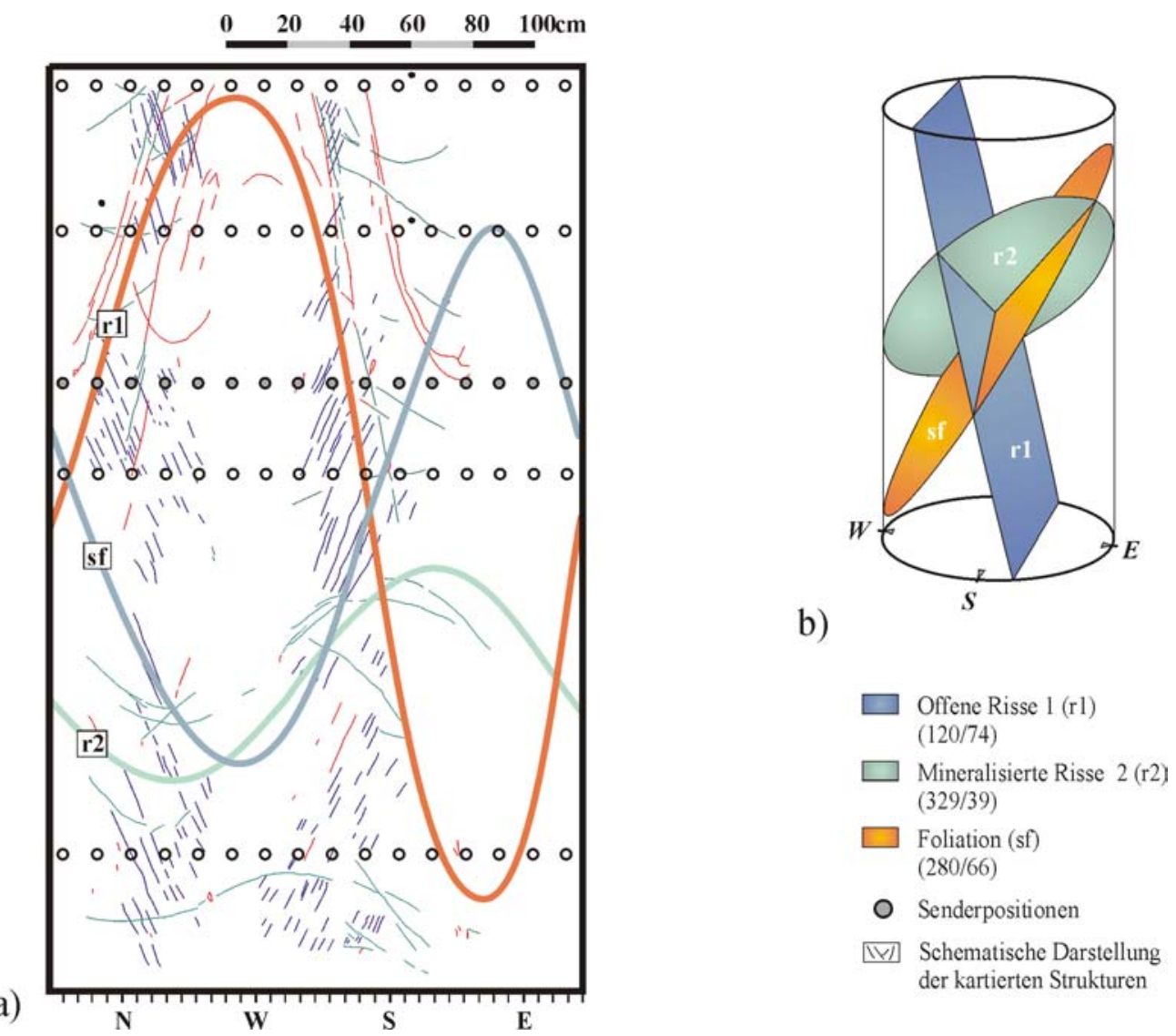

Abb. 8.6: Kartierung von Gefügeelementen der untersuchten Säule aus Prieborner Marmor. a) Darstellung der kartierten Strukturen und b) schematische Darstellung der Hauptgefügeelemente und ihre Orientierung innerhalb der Säule.

\subsubsection{Tomographische Inversion der Ultraschalldaten}

Für die tomographische Auswertung wurden Ultraschalluntersuchungen an fünf Ebenen der Prieborner Säule in den Höhen 0,58 m, 2,20 m, 3,23 m, 3,63 m und 3,88 m durchgeführt. Die für die Kompressionswellen in Abb. 8.7 dargestellten rekonstruierten Tomogramme zeigen untereinander eine relativ gleichmäßige Geschwindigkeitsverteilung. Danach befindet sich dezentriert nach Osten ein Geschwindigkeitsminimum, wohingegen im westlichen Teil der Säule ein lokales Geschwindigkeitsmaximum detektiert werden kann. Die Geschwindigkeiten variieren dabei zwischen $3,0 \mathrm{~km} / \mathrm{s}$ und 4,8 km/s. Die Säulenquerschnitte in den Höhen 0,58 m und $2,20 \mathrm{~m}$ erreichen lediglich maximal um $4.0 \mathrm{~km} / \mathrm{s}$. Eine Ausnahme bildet der Querschnitt in Höhe 3,63 m mit relativ geringen Geschwindigkeitskontrasten.

Der Niedriggeschwindigkeitsbereich könnte mit einer von GRUNERT (1988) diskutierten Kernauflockerung, bedingt durch die eventuell höhere Luftfeuchtigkeit auf der seezugewandten Seite erklärt werden. Beim Vergleich der ermittelten Gefügedaten der Säulenkartierung mit den Tomogrammen erscheint diese Erklärung nicht schlüssig. Hier wäre vielmehr eine $\mathrm{N}-\mathrm{S}$ streichende Niedriggeschwindigkeitszone $\mathrm{zu}$ erwarten, welche durch die makroskopisch nachweisbaren offenen Risse C1 erzeugt werden. 

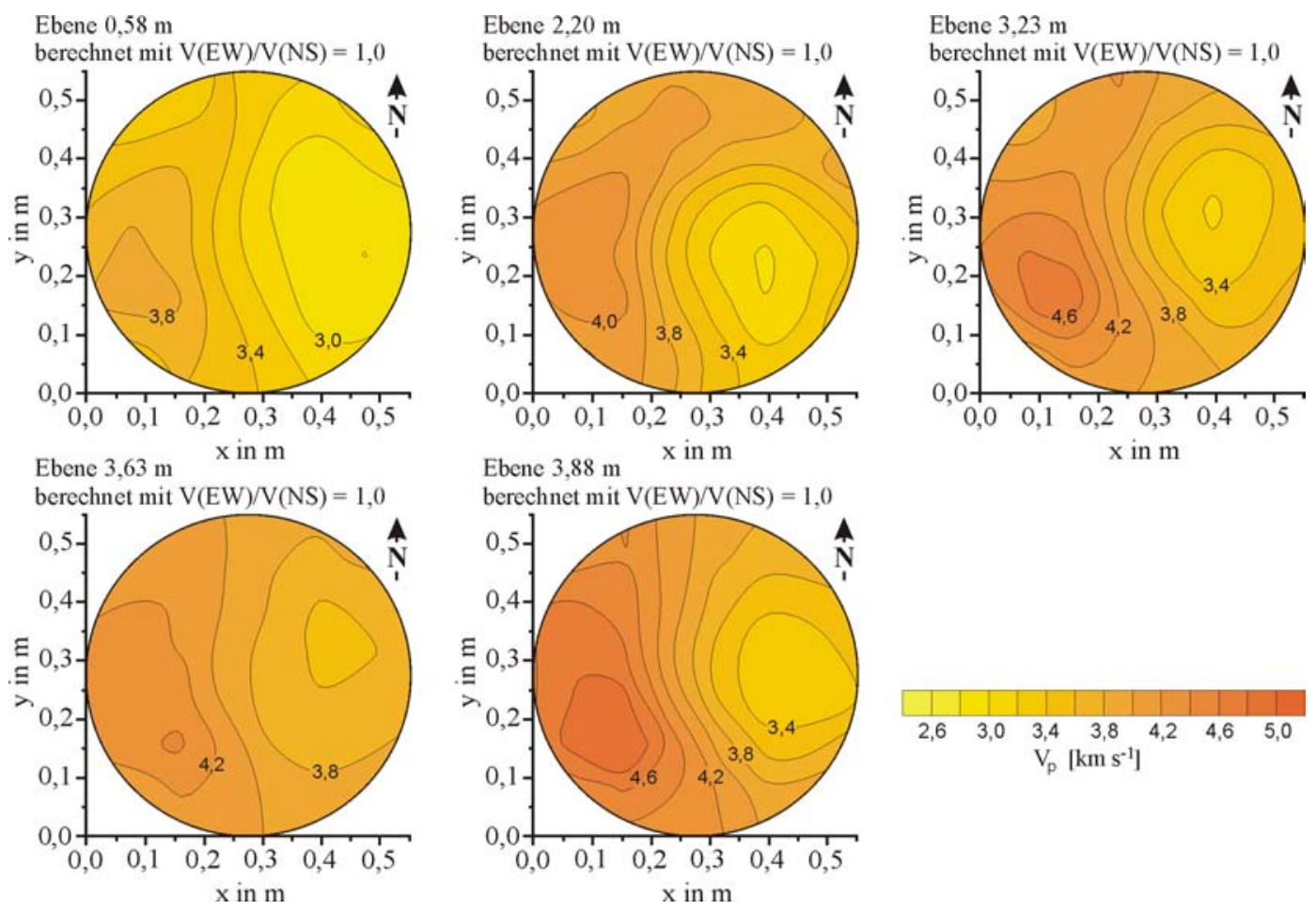

Ebene $3,88 \mathrm{~m}$
berechnet $\mathrm{mit} \mathrm{V}(\mathrm{EW}) / \mathrm{V}(\mathrm{NS})=1,0$
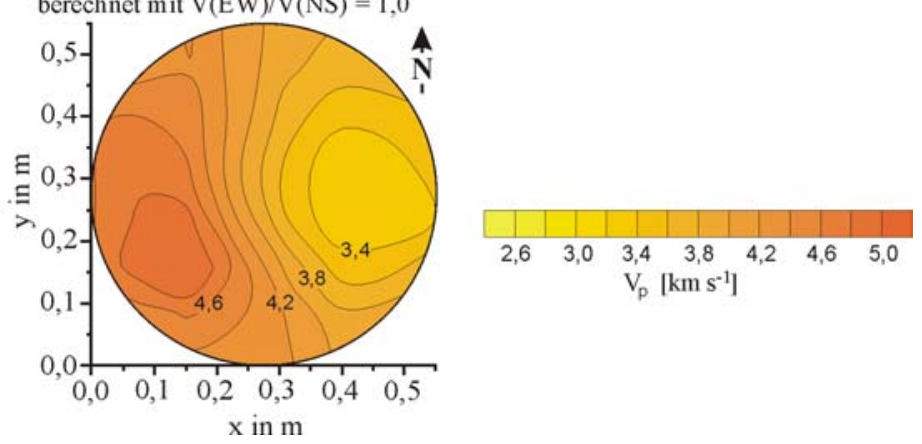

Abb. 8.7: Tomographische Inversionen der $\mathrm{V}_{\mathrm{p}}$-Ultraschalldaten in den verschiedenen Ebenen der Prieborner Marmorsäule.

\subsubsection{Labordaten vs. Felddaten}

Um zu überprüfen, wie die in Marmoren häufig vorkommenden Geschwindigkeitsanisotropien das Muster der Geschwindigkeitsverteilungen in den Tomogrammen beeinflussen bzw. verändern, wurden die vor Ort Daten den Labordaten gegenübergestellt. Die vollständige $\mathrm{V}_{\mathrm{p}}$-Bestimmung an Kugelproben (vgl. Kap. 4.3.3.3) hat gezeigt, dass eine deutliche intrinsische Richtungsabhängigkeit der Wellengeschwindigkeiten in Marmoren durch die Textur des Gesteins erzeugt wird. Diese Anisotropie wird i.d.R. aufgrund von Mikrorissbildung im Laufe der fortschreitenden Verwitterung verstärkt.

Die zum Vergleich herangezogene Probe aus Prieborner Marmor weist eine sehr starke Richtungsabhängigkeit der Ultraschallwellengeschwindigkeiten auf. In Abb. 8.8 ist dies für die wassergesättigte und trockene Probe dargestellt. Die $V_{p}$-Daten wurden aus Kugelmessungen gewonnen, wobei die Entnahme der Daten auf einer Schnittebene erfolgte, die eine vergleichbare Orientierung $\mathrm{zu}$ den Tomographieebenen vor Ort aufweist (ca. $70^{\circ}$ einfallende und geographisch gleich orientierte Foliation).

Das Diagramm zeigt, dass die $\mathrm{V}_{\mathrm{p}}$-Geschwindigkeiten zwischen den beiden Probenzuständen stark variieren. Die wassergesättigte Probe zeigt Werte zwischen $5,3 \mathrm{~km} / \mathrm{s}$ und $5,7 \mathrm{~km} / \mathrm{s}$ und die trockene Probe variiert von $2,2 \mathrm{~km} / \mathrm{s}$ bis $3,0 \mathrm{~km} / \mathrm{s}$. Für beide Probenzustände kann das Maximum parallel zur NS-Richtung und die minimalen $\mathrm{V}_{\mathrm{p}}$-Werte parallel zur EW-Richtung festgestellt werden. Die Verstärkung der Anisotropie von $5 \%$ im wassergesättigten Zustand $\mathrm{zu} 30 \%$ im trockenen Zustand bei gleicher Richtung zeigt, dass die Rissbildung die Richtungsabhängigkeit von $\mathrm{V}_{\mathrm{p}}$ verstärkt hat (vgl. Kap. 4.3.3.4). 


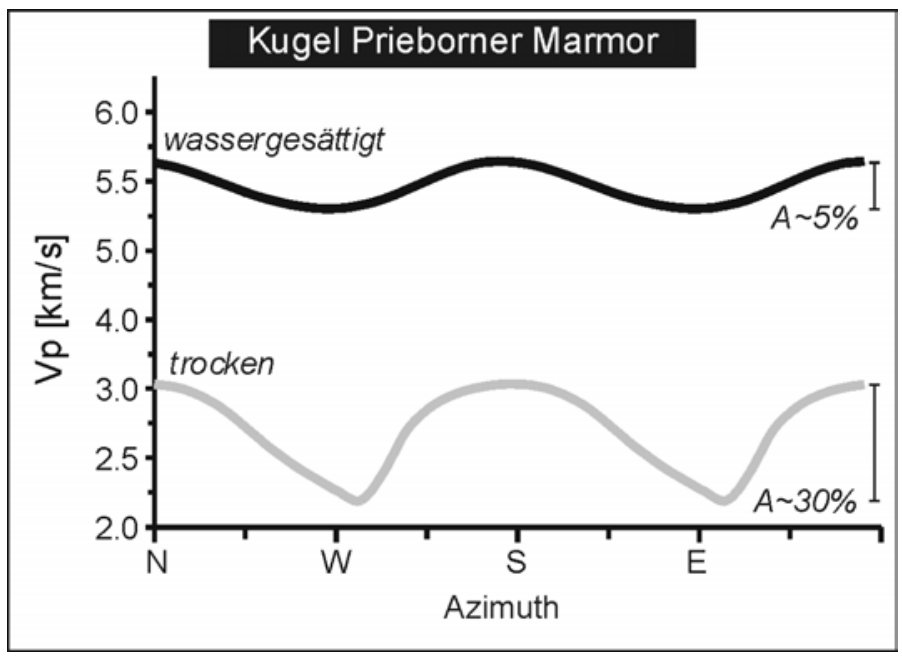

Abb. 8.8: Ultraschallgeschwindigkeitsanisotropie einer Kugelprobe des Prieborner Marmors im wassergesättigten (schwarze Linie) und im trockenen Probenzustand (graue Linie). Die jeweilige Geschwindigkeitsanisotropie ist in Prozent angegeben.

Um festzustellen ob diese ausgeprägte $V_{p}$-Anisotropie ein Tomogramm verändern kann, wurden die Kugeldaten aus der angeführten Ebene verwendet. Aus Ihnen selbst wurde ein Tomogramm erstellt. Hierzu wurden die Daten der Kugel im trockenen Probenzustand verwendet, da sie der Situation vor Ort am nächsten kommen. Die Geschwindigkeiten der Kugelprobe liegen zwar etwas niedriger als die der vor Ort gemessenen Säule. Dies kann jedoch auf i) einen abweichenden Verwitterungszustand sowie auf (ii) die Exposition am Bauwerk (konstruktives Bauelement) oder (iii) erhöhte Gesteinsfeuchte vor Ort zurückgeführt werden. Da die Strahlengänge der Kugelmessungen grundsätzlich durch den Mittelpunkt der Probe verlaufen, wurden sie für die tomographische Inversion umgerechnet. Dies erfolgte durch Abgreifen von Parallelstrahlen zu denen der Messanordnung der vor Ort Tomographie (s. Abb. 8.9a).

Grundsätzliche Voraussetzung für diese Vorgehensweise ist die Annahme eines gleichbleibenden bzw. gleich geschädigten Probenkörpers. Die umgewandelten Daten wurden somit vergleichbar $\mathrm{zu}$ denen der Säulenquerschnittstomogramme ausgewertet. Das so ermittelte Tomogramm ist in Abb. 8.9c dargestellt und zeigt eine deutliche Anisotropie der Ultraschallgeschwindigkeitsverteilung. Die jeweiligen Maxima sind auf der N- und S-Seite, die Minima entsprechend im Westen und Osten des Diagramms lokalisiert. Im Zentrum befindet sich ein weiterer Kanal mittlerer Geschwindigkeiten. Das Muster des Tomogramms weist dabei eine ausgeprägte Übereinstimmung mit dem der Kugelmessung auf (vgl. Abb. 8.9b mit Abb. 8.9c). Die Untersuchungen zeigen, dass die anisotrope Geschwindigkeitsverteilung auf die tomographische Auswertung einen Einfluss haben muss.

Aufgrund dieser Erkenntnis wurde die tomographische Inversion der Ultraschalldaten zusätzlich mit einer Einberechnung der Richtungsabhängigkeit ausgewertet. Dies erfolgte durch ein Anisotropieverhältnis von $\mathrm{V}_{\mathrm{p}}$-Ost-West $\left(\mathrm{V}_{\mathrm{EW}}\right) \mathrm{zu} \mathrm{V}_{\mathrm{p}}$-Nord-Süd $\left(\mathrm{V}_{\mathrm{NS}}\right)$. Dieses wurde entsprechend der bekannten Richtungsabgängigkeit in Bezug zur Orientierung der Foliation eingerechnet. Die entsprechenden Ergebnisse zunehmender Anisotropie sind in Abb. 8.10 exemplarisch für die Ebene auf 3,23 m für $\mathrm{V}_{\mathrm{EW}} / \mathrm{V}_{\mathrm{NS}}=0,9,=0,8,=0,7$ und $=0,6$ dargestellt. Deutlich ist $\mathrm{zu}$ erkennen, dass sich mit zunehmendem Anisotropieverhältnis die 
Geschwindigkeitsverteilung in den Tomogrammen ändert. Hierbei entwickelt sich das lokale Geschwindigkeitsminimum zunehmend zu einem NS-streichenden Niedriggeschwindigkeitskanal. Das lokale Geschwindigkeitsmaximum verschwindet mit zunehmendem Anisotropieverhältnis.

a)
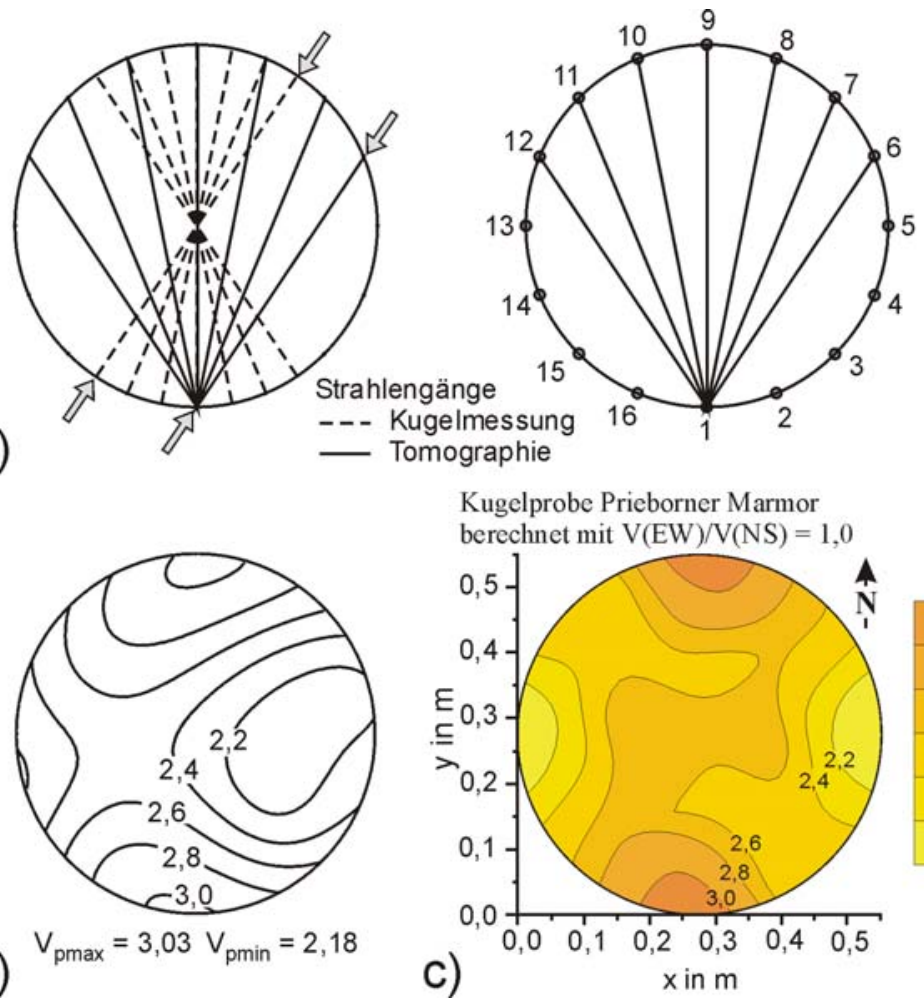

Kugelprobe Prieborner Marmor

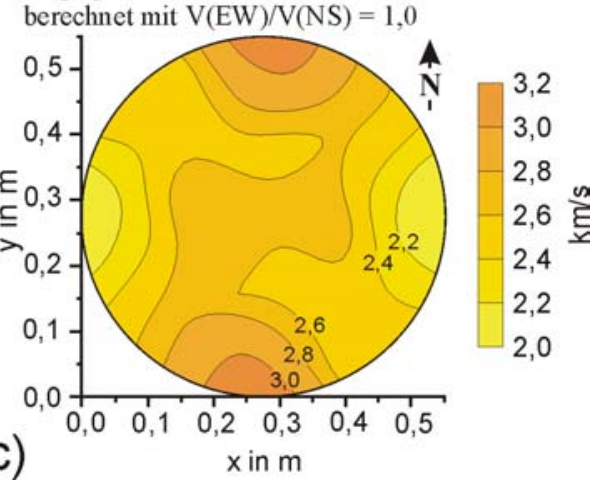

Abb. 8.9: Überführung der Ultraschallgeschwindigkeitsverteilung aus Kugelmessungen des Prieborner Marmors in eine tomographische Inversion: a) Durchschallungsstrahlen, die das Zentrum der Kugel durchstoßen und in die vor Ort Strahlengeometrie überführt wurden, b) Geschwindigkeitsverteilung in einer Kugelprobe aus Prieborner Marmor (Schmidt'sches Netz, untere Halbkugel) und c) entsprechende Geschwindigkeitsverteilung in der ultraschalltomographischen Darstellung.

Das Anisotropieverhältnis von $\mathrm{V}_{\mathrm{EW}} / \mathrm{V}_{\mathrm{NS}}=0,6$ entspricht einer Richtungsabhängigkeit von ca. $40 \%$ und erscheint aufgrund der Erkenntnisse aus den Kugelmessungen zu hoch. Jedoch ist auch in den Tomogrammen mit einem Anisotropieverhältnis von $\mathrm{V}_{\mathrm{EW}} / \mathrm{V}_{\mathrm{NS}}=0,8$ und $=0,7$ ein deutlicher Niedriggeschwindigkeitskanal mit NS-Streichen erkennbar. Parallel zu dieser Richtung verläuft ebenfalls das Streichen der durch die Gefügekartierung ermittelten Makrorisse. Die Vermutung liegt nahe, dass diese Diskontinuitäten im Gestein eine Reduktion der Geschwindigkeiten hervorrufen. Damit kann eine weitgehende Übereinstimmung zwischen Gefügeparametern und Geschwindigkeitsverteilung festgestellt werden.

Im Prinzip wurden bei der oben vorgestellten Vorgehensweise die intrinsischen und mikrorissgenerierten Geschwindigkeitsanisotropien des Marmors aus den ermittelten Geschwindigkeitsverteilungen herauskorrigiert. Der Grundgedanke ist dabei, dass intrinsiche Anisotropien aber auch gleichmäßig verteilte anisotrope Schäden (in diesem Fall eine anisotrope Mikrorissverteilung) weitere zumeist lokale Entfestigungsbereiche überdecken 
bzw. verzerren (maskieren). Da insbesondere die lokalen Schäden wie Makrorisse oder auch unterschiedliche Randentfestigungen (beregnete / geschützte Bauteilseite) mittels Ultraschalltomographie detektiert werden sollen, erscheint es notwendig bei deutlich anisotropen Gesteinen die Richtungsabhängigkeit von Ultraschallgeschwindigkeiten zu beachten, da es ansonsten zu Fehldiagnosen kommen kann.
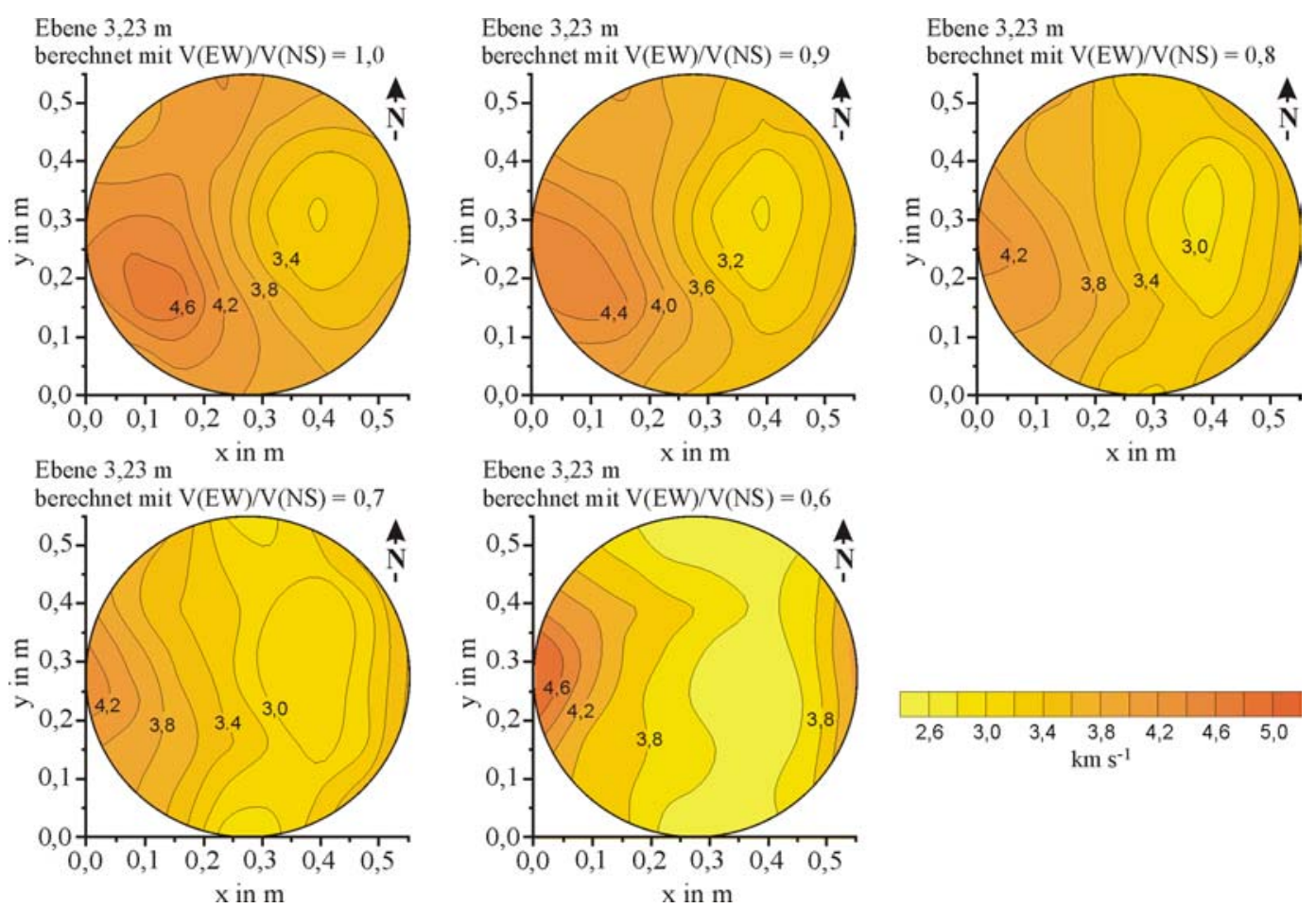

Abb. 8.10: Tomographische Inversion von Ultraschallgeschwindigkeiten des Prieborner Marmors (Ebene 3,23 m). Auf Laufzeitmessungen basierende Tomogramme der vor Ort ermittelten $V_{p}$ Daten mit Einrechnung zunehmender Anisotropie bis ungefähr $40 \%\left(\mathrm{~V}_{\mathrm{EW}} / \mathrm{V}_{\mathrm{NS}}=0,6\right)$. Die Geschwindigkeiten sind in $\mathrm{km} \mathrm{s}^{-1}$ angegeben. Die Nordrichtung ist gekennzeichnet.

\subsubsection{Kauffunger Marmorsäule}

\subsubsection{Schadenskartierung und Makro / Mikrogefüge}

Die untersuchte Kauffunger Marmorsäule ist Teil der Säulenkolonnade der Innenhofseite des nördlichen Seitenflügels des Marmorpalais. Es handelt sich ebenfalls um eine tragende Säule, die mit weiteren neun Säulen den Architrav stützt (s. auch Abb. 8.1b). Der rötliche sehr heterogen erscheinende Kauffunger Marmor besteht aus einer grauen feinkörnigen CalcitMatrix, welche von gelben bis gelbrötlichen feinkörnigen Dolomitadern durchzogen ist. An der untersuchten Säule ist eine, anhand eines deutlichen Farbkontrasts gut zu erkennende, Population von Dolomitadern parallel zur Foliation entwickelt. Die Größe der Adern variiert von $\mathrm{mm}$ bis $\mathrm{zu}$ einigen $\mathrm{cm}$ und weist sehr unregelmäßige Formen auf. Der Gehalt des Dolomits liegt bei ungefähr $10 \%$ des Gesamtvolumens. Aufgrund der höheren Verwitterungsresistenz, formt der Dolomit ein positives Relief auf der Oberfläche der verwitterten Säule. Die Foliation streicht N-S und weist ein Einfallen nach W auf (280/66, vgl. Abb. 8.11). 


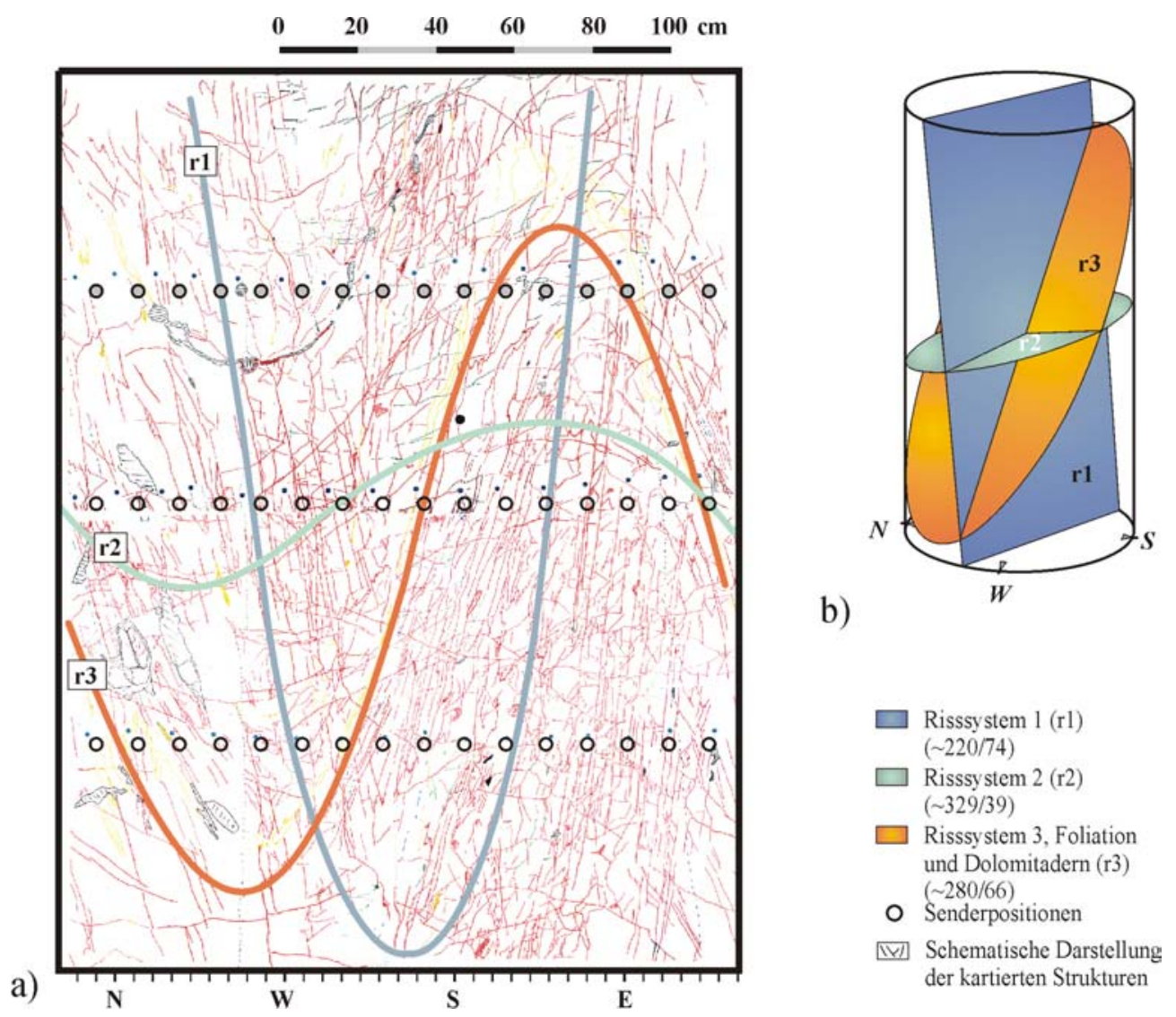

Abb. 8.11: Kartierung von Gefügeelementen der untersuchten Säule aus Kauffunger Marmor. a) Darstellung der kartierten Strukturen und b) schematische Darstellung der Hauptgefügeelemente und ihre Orientierung innerhalb der Säule.

Der Kauffunger Marmor zeigt schon makroskopisch ein vollständig anderes Verwitterungsverhalten als der Prieborner Marmor. Während letzterer ein feinkörniges Abzuckern der Oberflächen zeigt, was auf einen vollständigen und penetrativen Verlust der Gefügekohäsion hinweist, verwittert der Kauffunger Marmor über präexistierende Makround Mikrorisse. Daher sollen sich die weiteren gefügekundlichen Betrachtungen des Kauffunger Marmors auf seine Risssysteme in den verschiedenen Skalenbereichen beziehen.

\section{- Makrorisse}

Der Marmor wird makroskopischen durch verschiedene parallel angelegte Risssysteme geprägt, welche mittels Gefügekartierungen erfasst wurden und in Abb. 8.11 schematisch dargestellt sind. An der untersuchten Säule können sowohl offene Risse als auch mit verschiedenen Mineralphasen versiegelte Risse beobachtet werden. Letztere variieren von einigen $\mathrm{mm} \mathrm{zu} \mathrm{cm} \mathrm{Größe} \mathrm{und} \mathrm{sind} \mathrm{vorwiegend} \mathrm{mit} \mathrm{unterschiedlich} \mathrm{gekörntem} \mathrm{Calcit}$ mineralisiert. Untergeordnet ist auch Dolomit und ein feinverteiltes kräftig rotes Mineral beteiligt, bei dem es sich vermutlich um Hämatit handelt. Die meisten der versiegelten Risse sind rückgewittert, formen also ein negatives Relief (s. Abb. 8.12), was auf eine höhere Witterungssensitivität schließen lässt. Die Rückwitterungsrate beträgt hierbei einige $\mathrm{mm}$. Hingegen ist die Tiefe der offenen Risse in das Säuleninnere unklar. Zwischen den offenen Rissen können Materialausbrüche beobachtet werden. Drei Rissgenerationen sind voneinander abzugrenzen. Rissgeneration r1 fällt steil nach SW ein ( 220/74), wohingegen 
Rissgeneration r2 ein mittleres Einfallen nach NNW aufweist $(\sim 329 / 39)$. Die dritte Generation r3 verläuft parallel zur Foliation und fällt nach W ein ( 280/66). Für alle drei Risssysteme ist keine bevorzugte Ausbildung von offenen oder versiegelten Rissen feststellbar. Hinsichtlich der Ultraschalluntersuchungen kann davon ausgegangen werden, dass die offenen Risse den größten Einfluss auf die elastischen Eigenschaften haben, da sie wesentliche Diskontinuitäten des Gesteins darstellen.

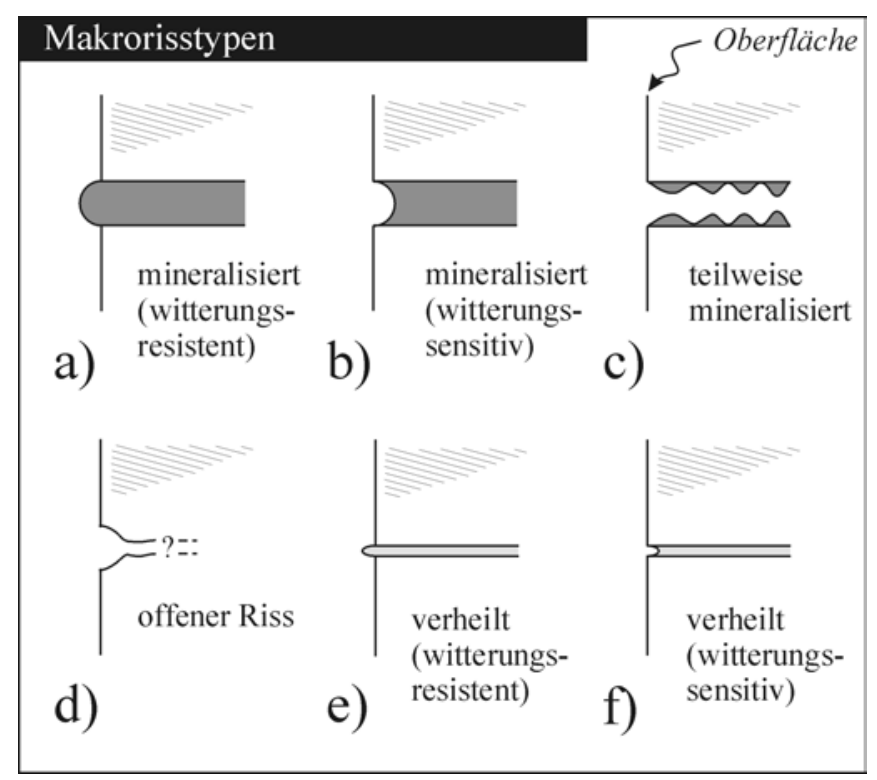

Abb. 8.12: Schematische Darstellung von Rissen des Kauffunger Marmors, welche makroskopisch zu beobachten sind und mikroskopischen Risstypen zugeordnet werden können.

\section{- Mikrorisse}

Das Mikrorissgefüge des Kauffunger Marmors ist komplex entwickelt. Neben verheilten und versiegelten bzw. mineralisierten präexistierenden Rissen können auch offene Risse detektiert werden. Das gesamte Rissinventar des Kauffunger Marmors wurde im Rahmen einer Diplomarbeit (RICHTER, 2000) an orientierten Bohrkernen mehrerer Säulen detailliert aufgenommen. Die wesentlichen gefügekundlichen Ergebnisse sind im Folgenden dargestellt.

\section{$\underline{\text { Versiegelte Mikrorisse }}$}

Die versiegelten Mikrorisse können nach mikroskopischen Untersuchungen in zwei Typen, einen feinkörnigen und einen grobkörnigen versiegelten Risstyp, untergliedert werden. Für den feinkörnigen Risstyp sind deutlich geringere Korngrößen der Mineralisate gegenüber dem Muttergestein festzustellen. Nur lokal können in den Zentren der Risse auch größere, zumeist ausgelängte, bis $100 \mu \mathrm{m}$ große Körner beobachtet werden. Das KL-Inventar weist auf nur sehr untergeordnete Beteiligung von Dolomit an der Versiegelung hin. Akzessorisch sind auch Eisenoxide vorhanden. Hinsichtlich ihrer Orientierung können zwei Rissscharen unterschieden werden, wobei eine parallel, die andere senkrecht zur Foliation verläuft.

Der grobkörnig mineralisierte Risstyp ist zumeist durch größere Korngrößen gekennzeichnet, wobei hier Dolomit einen höheren Anteil am Volumen mit ca. 20 \% hat. Sowohl die Calcitals auch die Dolomit-Kristalle weisen vorwiegend polygonale Kornformen und häufige 
Interstrukturierung in Form von Zwillingen und geöffneten Spaltflächen auf. Auffällig ist ein häufiges Aufreißen der Risse an der Grenze zwischen Muttergestein und versiegeltem Riss. Dies zeigt an, dass vor allem die versiegelten Risse selektiv während der Verwitterung reaktiviert wurden.

Die versiegelten Mikrorisse konnten aufgrund ihrer Weitständigkeit, von i.d.R. über $5 \mathrm{~cm}$, mikroskopisch nicht statistisch erfasst werden. Die Daten können jedoch aus den Schadenskartierungen der Säule entnommen werden (s.o.).

\section{$\underline{\text { Verheilte Mikrorisse }}$}

Die verheilten Risse sind durch ihr typisch syntaxiales Anwachsen am umgebenen Gestein gekennzeichnet und nur mittels KLM detektierbar. Sie sind durch ein komplexes und in der Häufigkeit stark variierendes Auftreten gekennzeichnet. Vielfach ist ein spindelförmiges Auffächern und folgendes Zusammenlaufen $\mathrm{zu}$ beobachten. Die Ergebnisse einer semiquantitativen Analyse der bevorzugten Orientierung dieser Risse mittels KLM an Dünnschliffen in drei Raumlagen ist in Abb. 8.13 dargestellt. Dabei sind drei orthogonale Risssysteme auszumachen, von denen eines der Foliation folgt. Zusätzlich können zwei zur Foliation diagonal verlaufende Risssysteme ermittelt werden.
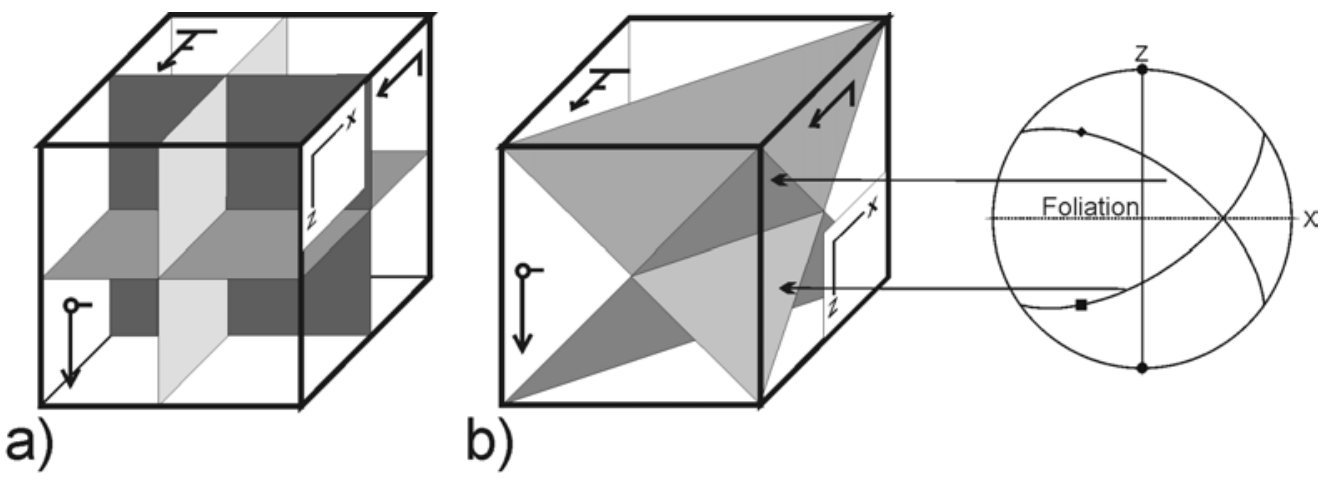

Abb. 8.13: Schematische Darstellung der mittels KLM analysierten bevorzugten Orientierung verheilter Risssysteme im Kauffunger Marmor; a) Risssysteme parallel und senkrecht und b) diagonal zur Foliation (Schmidt'sches Netz, untere Halbkugel).

Im Gegensatz zu den versiegelten Rissen kann keine Reaktivierung dieser Risse in Form einer Öffnung beobachtet werden. Es ist davon auszugehen, dass ihr Einfluss auf die Ultraschallgeschwindigkeiten nur von untergeordneter Bedeutung ist .

\section{Offene Mikrorisse}

Eine statistische Erfassung von offenen intragranularen Mikrorissen erfolgte mittels U-Tisch Mikroskopie an Dünnschliffen in drei Schnittlagen. Die intragranularen Risse kommen bevorzugt in großen Altkörnern vor. Die in Abb. 8.14 aufgeführten Daten repräsentieren 614 gemessene Risse aus 112 Kristallen in drei Schnittlagen und sind in Form einer flächentreuen Projektion dargestellt. Dabei können drei Rissscharen unterschieden werden, die in Abb. 8.14b anhand der makroskopischen Gefügeelemente der Foliation und Makrorisse schematisch in die Säule vor Ort rückorientiert wurden. Zwei dominierende Risssysteme fallen nach Westen ein und Verlaufen annähernd parallel zur Foliation. Ein untergeordnete System intragranularer Mikrorisse weist ein Einfallen nach SE auf. Um zu überprüfen, nach 
welcher Kristallfläche die Mikrorisse bevorzugt ausgebildet sind, wurde zusätzlich der Winkel zwischen gemessener Fläche und c-Achse des entsprechenden Kristalls ausgewertet (s. Abb. 8.14c; bezüglich des Verfahrens vgl. TURNER, 1954). Die Auswertung zeigt, dass die meisten Risse mit der e-Fläche $\{018\}$ übereinstimmen und nur untergeordnet die r- $\{012\}$ sowie die f-Flächen $\{104\}$ aktiviert wurden.

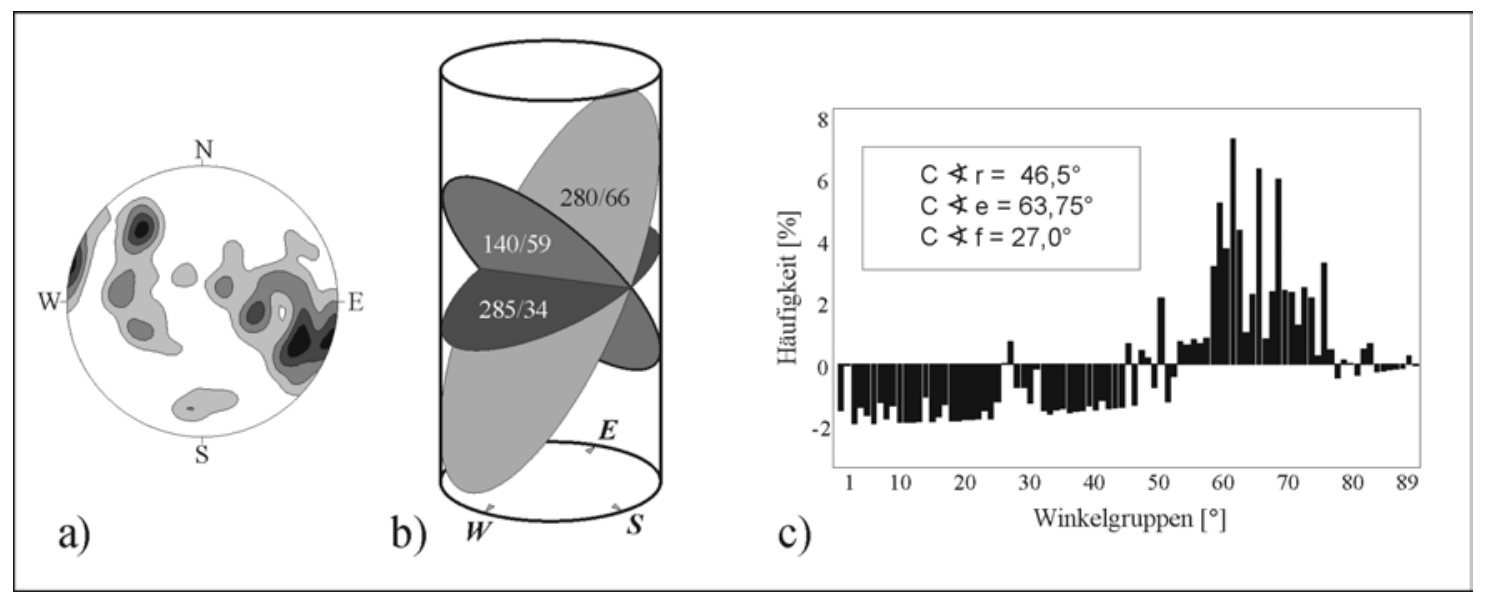

Abb. 8.14: Bevorzugte Orientierung von intragranularen Mikrorissen im Kauffunger Marmor ermittelt an Bohrkernen einer ausgewählten Säule: a) bezogen auf die geographischen Koordinaten (Belegungsdichtediagramm, Schmidt'sches Netz, untere Halbkugel), b) schematische Darstellung der gemessenen Risse innerhalb der untersuchten Säule und c) Diagramm der Häufigkeit von Winkeln zwischen den beobachteten intragranularen Mikrorissen und der cAchse des jeweiligen Kristalls.

\subsubsection{Tomographische Inversion der Ultraschalldaten}

Die Ultraschallanalysen an der Säule aus Kauffunger Marmor wurden an drei horizontalen Ebenen in den Höhen 0,92 m, 1,59 m und 2,12 m, ausgehend von der Oberfläche des Säulenunterlagernden Profils, durchgeführt. In Abb. 8.15 sind die Ergebnisse der tomographischen Inversion für die gemessenen Kompressions- und Scherwellenlaufzeiten dargestellt. Die Tomogramme zeigen ein heterogeneres Bild im Vergleich zu denen der Prieborner Marmorsäule. Für die beiden unteren Säulenebenen ist ein Minimum mit $V_{p}=3,4 \mathrm{~km} / \mathrm{s}$ und $\mathrm{V}_{\mathrm{s}}=2,1 \mathrm{~km} / \mathrm{s}$ im Zentrum des Querschnitts zu ermitteln. Die maximalen Geschwindigkeiten liegen im Bereich $\mathrm{V}_{\mathrm{p}}=5,2 \mathrm{~km} / \mathrm{s}$ und $\mathrm{V}_{\mathrm{s}}=3,1 \mathrm{~km} / \mathrm{s}$.

Die $\mathrm{V}_{\mathrm{p}}$-Geschwindigkeitsverteilung in der Ebene auf 2,12 $\mathrm{m}$ zeigt ein homogeneres Muster mit $\mathrm{V}_{\mathrm{p}}$-Werten zwischen $3,8 \mathrm{~km} / \mathrm{s}$ und $5,2 \mathrm{~km} / \mathrm{s}$. Generell kann eine Niedriggeschwindigkeitszone, welche von SW nach NE verläuft, beobachtet werden. Im NW und im SE befinden sich hingegen Zonen höherer Geschwindigkeit. Ein vergleichbares Verteilungsmuster ist für die tomographische Auswertung der $\mathrm{V}_{\mathrm{s}^{\mathrm{s}}}$-Werte festzustellen. Dabei befinden sich die $\mathrm{V}_{\mathrm{s}}$-Werte zwischen $2,5 \mathrm{~km} / \mathrm{s}$ und 3,3 km/s. 

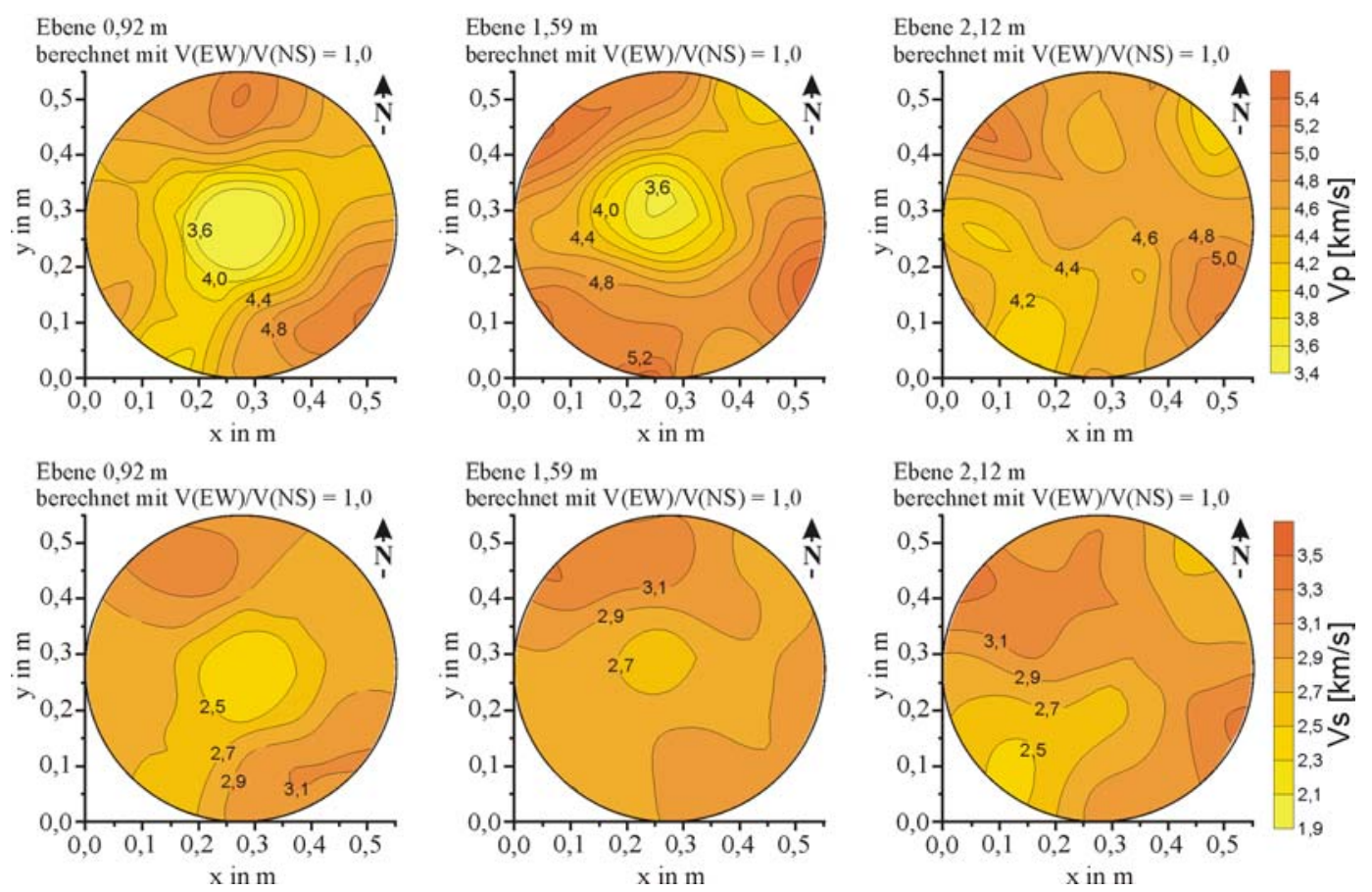

Abb. 8.15: Tomographische Inversionen der $\mathrm{V}_{\mathrm{p}^{-}}$(obere Reihe) und $\mathrm{V}_{\mathrm{s}}$-Ultraschalldaten (untere Reihe) in den verschiedenen Ebenen der Kauffunger Marmorsäule.

\subsubsection{Labordaten vs. Felddaten}

Der Kauffunger Marmor zeigt ein deutlich abweichendes Verwitterungsverhalten im Vergleich zum Prieborner Marmor. Für den Kauffunger Marmor ist dabei eine bevorzugte Entfestigung entlang präexistierender Risssysteme festzustellen und nur untergeordnet eine penetrative inter- und/oder intrakristalline Rissbildung. Dabei kann anhand der mikroskopischen Analysen davon ausgegangen werden, dass der größte Anteil der Ultraschallgeschwindigkeitsherabsetzung durch die Reaktivierung der versiegelten Risse hervorgerufen wird.

Um zu überprüfen, inwieweit die Beobachtungen aus den makroskopischen Rissen der Säulenkartierungen mit den Ergebnissen der Tomographie übereinstimmen, wurden die versiegelten/mineralisierten Risse quantitativ ausgewertet. Dazu wurden in einem ersten Schritt die makroskopisch erfassbaren Risse in ein Risshäufigkeitsdiagramm überführt. Das heißt, dass auf einer Traverse in einem Säulenabschnitt vergleichbar zur Ebene der Tomographie die Anzahl der Risse aufgenommen wurde. Das Risshäufigkeitsdiagramm in Abb. 8.16a zeigt die gemittelten Rissabstände und das Streichen im Säulenanschnitt für die drei Risssysteme C1, C2 und C3. Die so gewichteten Rissdaten wurden im Folgenden in ein 3 D-Häufigkeitsdiagramm überführt (Schmidt'sches Netz, untere Halbkugel). Dieses zeigt die Anzahl von Rissen für ein Linear (Durchschallungstrecke), welches das Zentrum der Kugel durchsticht (für Details dieser Methode s. VOLLBRECHT et al. 1994 oder RASOLOFOSAON et al. 2000). Das Maximum des Risshäufigkeitsdiagramms repräsentiert die Richtung der größten Risshäufigkeit. Das bedeutet für das Rissmaximum I, dass ein Strahl in diese Richtung auf 21 Risse trifft. 


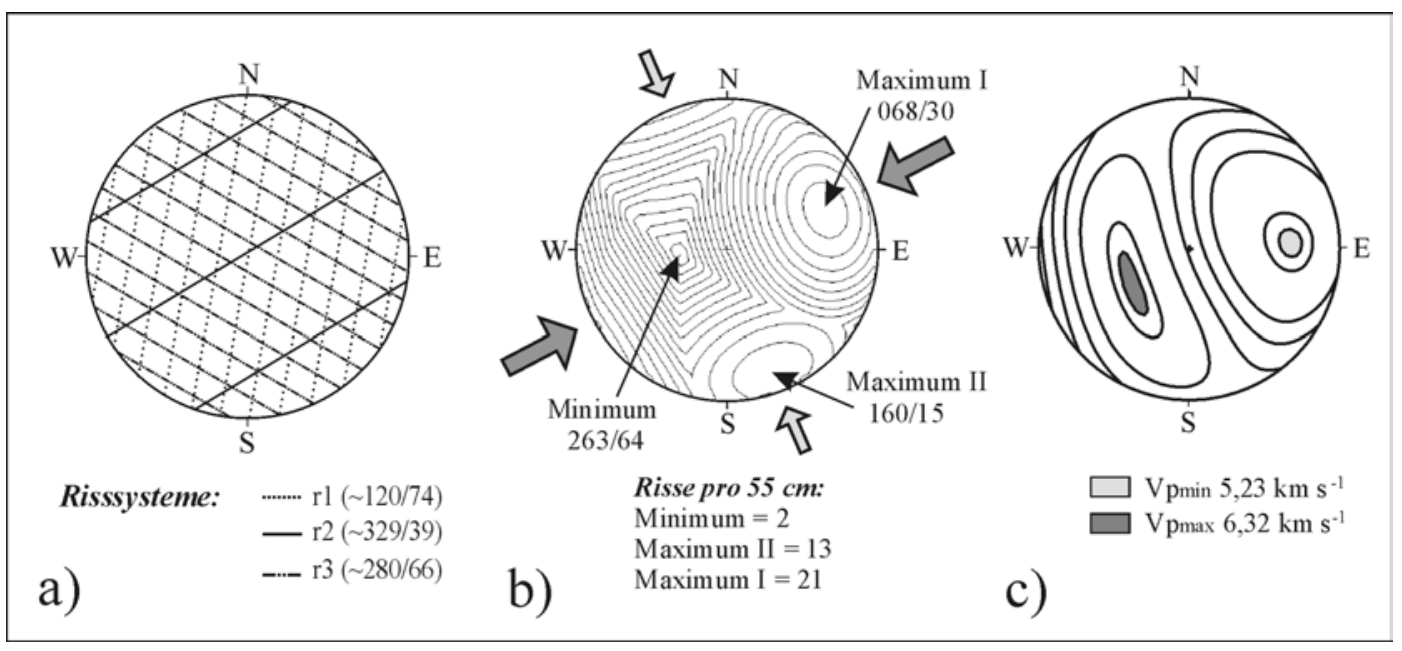

Abb. 8.16: a) Schematische Darstellung der aus den Kartierungen ermittelten Risssysteme als Schnittlinien mit der Horizontalen der untersuchten Säule, b) Rissdichtendiagramm zeigt die Anzahl von Mikrorissen auf einer Linie, die durch das Zentrum einer Kugel verläuft und c) experimentell ermittelte $\mathrm{V}_{\mathrm{p}}$-Geschwindigkeitsverteilung des Kauffunger Marmors an einer ausgewählten Kugel (Schmidt'sches Netz, untere Halbkugel).

Das Risshäufigkeitsdiagramm wird dabei durch zwei Maxima dominiert. Das Minimum befindet sich leicht abweichend vom Zentrum. Damit ist die geringste Häufigkeit der Risse mehr oder minder parallel zur Längsachse der Säule festzustellen. Im Vergleich ist in Abb. 8.16c die Geschwindigkeitsverteilung einer Kugelprobe aus Kauffunger Marmor im trockenen Probenzustand dargestellt, wobei die Lage der Foliation die gleiche Orientierung wie in der gemessenen Säule besitzt. Beim direkten Vergleich von Abb. 8.16b mit Abb. 8.16c ist festzustellen, dass die niedrigsten Geschwindigkeiten parallel zum Rissmaximum I und die höchsten Geschwindigkeiten parallel zum Rissminimum auftreten. Jedoch ist der Rissabstand der makroskopisch einmessbaren Risse mit im Mittel von $50 \mathrm{~mm}$ in etwa gleichgroß wie die gemessene Kugelprobe. Somit kann ein nur geringer Einfluss dieser Risse auf die Kugelmessungen angenommen werden. Dies bedeutet aber gleichzeitig, dass das makroskopisch ermittelbare Rissmuster durch Herabsetzung der Geschwindigkeiten das Muster der $\mathrm{V}_{\mathrm{p}}$-Kugelverteilung verstärken sollte.

Beim Vergleich der Tomogramme in der Säulenebene bei 2,12 m (Abb. 8.15) mit dem Risshäufigkeitsdiagramm (Abb. 8.16) ist festzustellen, dass die niedrigsten Geschwindigkeiten mit dem Rissmaximum I korrelieren. Damit kann eine weitgehende Übereinstimmung mit den makroskopischen und mikroskopischen Beobachtungen sowie den Labormessungen festgestellt werden. Der Anteil der Geschwindigkeitsreduktion durch das jeweilige Risssystem bleibt jedoch unklar.

\subsection{Fallstudie: Möglichkeiten des Einsatzes der Volltränkung vor Ort}

Die Acrylharz-Volltränkung wird in der Denkmalpflege häufig angewandt um stark geschädigte Objekte aus Naturwerkstein zu konservieren. Das Imprägnierungsverfahren wird hierbei nicht vor Ort durchgeführt, so dass die Bauteile abgebaut und zur Tränkungsanlage transportiert werden müssen. Für Objekte die in Bauwerke integriert sind, ist ein Ausbau i.d.R. jedoch sehr aufwendig und damit kostenintensiv, wie z.B. für die Säulen des 
Marmorpalais, die z.T. über Stahlverbindungen mit überlagernden Bauteilen verbunden sind. Daher stellt sich die Frage ob und inwieweit eine Volltränkung bestimmter Objekte direkt am Bauwerk durchgeführt werden kann. Um dies zu überprüfen wurde eine Volltränkung unter Berücksichtigung der vor Ort Begebenheiten im Labor durchgeführt. Die Untersuchung erfolgte an einer zuvor thermisch entfestigten Probe aus Carrara Marmor.

Für den Volltränkungsversuch wurde eine zylindrische Probe des Marmors unter geringem einaxialem Druck in eine Gesteinspresse eingespannt. Als Imprägnierungsmittel diente ein speziell modifiziertes monomeres Methylmetacrylat (MMA). Das Einbringen des Kunststoffs in den Porenraum erfolgte durch Anlegen eines Vakuums. Die Polymerisation des Kunststoffes wurde durch Temperaturerhöhung eingeleitet.

Die Überprüfung des Eindringverhaltens und der Aushärtung des Kunstharzes wurde durch begleitende Ultraschallmessungen während des Tränkungsversuches detektiert. Zur qualitativen Ermittlung der festigenden Wirkung und zum Nachweis des Kunststoffes im Porenraum erfolgten REM-Untersuchungen an Bruchproben. Zusätzlich wurden die Porenraumeigenschaften vor und nach der Volltränkung ermittelt.

\section{- Versuchsaufbau}

Der Volltränkungsversuch erfolgte an einer zylindrischen Marmorprobe welche einen Durchmesser von $100 \mathrm{~mm}$ und eine Höhe von $150 \mathrm{~mm}$ aufweist. Für den Versuchsaufbau wurde der Zylinder axial in eine Hochdruckpresse eingespannt und an den Druckstempeln abgedichtet. Zum Anlegen des Vakuums und für die Zufuhr des Kunstharzes wurden zwei Rohrstutzen (Packer) angebracht (Abb. 8.17). Zur verbesserten Verteilung bzw. Vergrößerung der Ansatzfläche für das Tränkungsmittel dienten engmaschige Drahtgitter zwischen Probe und Packeraufsatz. Die äußere Abdichtung der Probe erfolgte durch einen Hartgummischlauch, welcher mit Schellen befestigt wurde. Die Wärmebehandlung und damit die Einleitung der Polymerisation wurde durch ein Heizband erreicht, welches mehrfach um den Dichtungsschlauch geführt wurde.

Für die Messung der Ultraschallgeschwindigkeiten befinden sich Schallgeber und -empfänger in den Stempeln der Presse. Dies ermöglicht die Durchschallung parallel zur Längsachse der Probe. Ermittelt wurden sowohl die Laufzeiten von Kompressions- als auch von Schwerwellen (1 MHz).

\section{- Verwendetes Probenmaterial}

Für den Volltränkungsversuch wurde eine Probe aus Carrara Marmor (Handelstyp Bianco CD) ausgewählt. Der weiße Marmor ist durch eine typische Marmorierung von unregelmäßigen, schlierenartigen dunklen Bändern charakterisiert. Im Mikrobereich weist die Probe ein equigranular polygonales Gefüge auf, mit vorwiegend geraden bis leicht geschwungenen Korngrenzen. Der Marmor zählt mit einer mittleren Korngröße von $130 \mu \mathrm{m}$ zu einer feinkörnigen Carrara Sorte. Die Textur ist mit einem c-Achsen Maximum von 1,46 VGV schwach ausgebildet (vgl. ONDRASINA et al., 2002). Weiteres Gefügemerkmal ist eine ausgeprägte Modifizierung der Korngrenzenflächen durch Lösungsgefüge, die auf eine Fluid-generierte Alteration in der Lagerstätte zurückgeführt werden kann. 

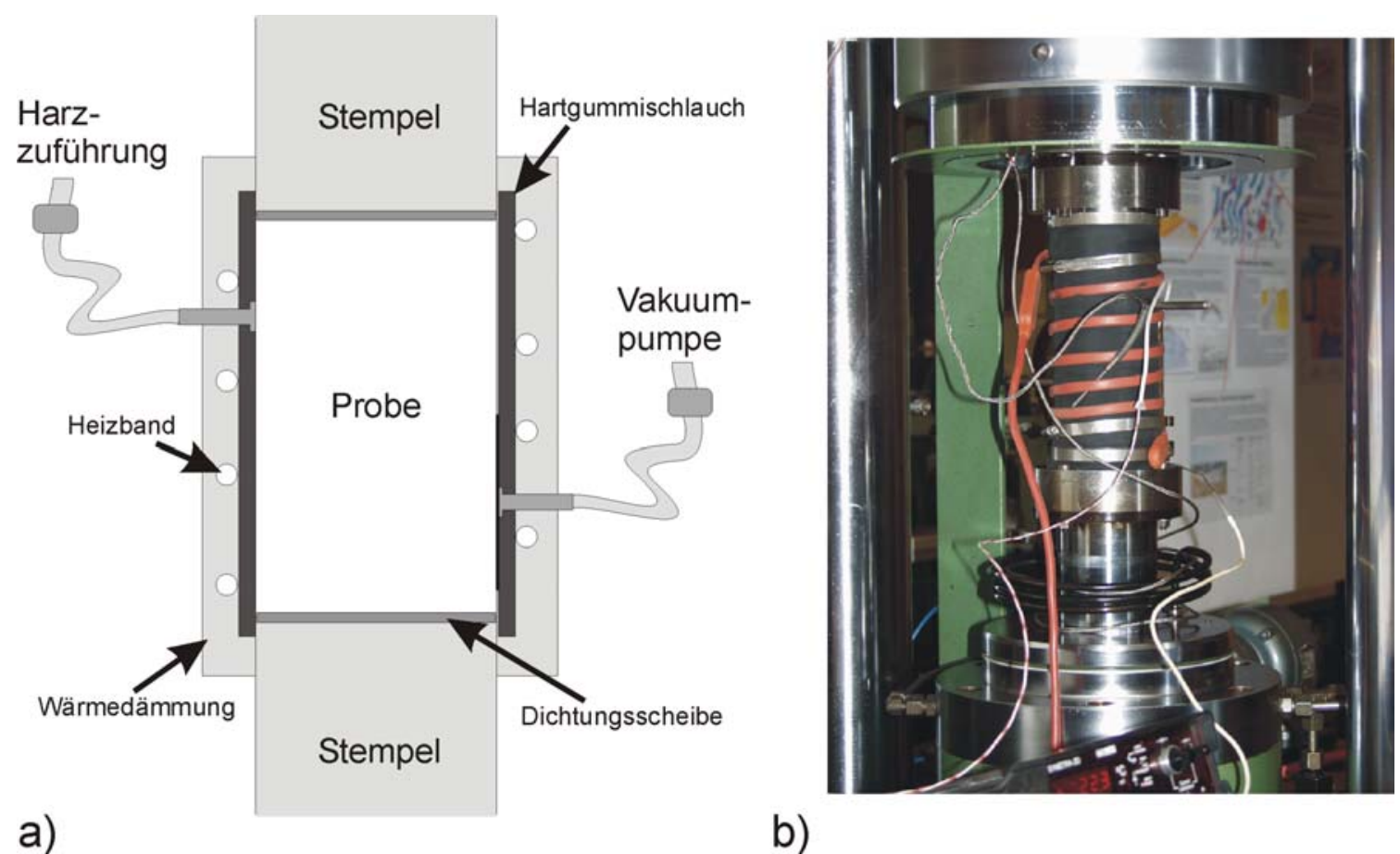

b)

Abb. 8.17: Versuchsaufbau der modellhaften in situ-Volltränkung: a) Schematische Darstellung des Versuchsaufbaus und b) zeigt die in die Presse eingespannte Probe mit Ummantelung und Heizband.

Die Probe wurde vor dem Volltränkungsversuch mechanisch durch eine Wärmebehandlung von $200{ }^{\circ} \mathrm{C}$ aufgelockert. Dadurch erfolgte eine Erhöhung der Porosität auf 0,74\%. Nach REM-Untersuchungen an Bruchproben führte die thermische Beanspruchung des Marmors zu einer gesteinsdurchdringenden Entfestigung an Korngrenzen.

\section{Verwendeter Kunstharz}

Als Kunstharz wurde ein monomeres Methylmetacrylat mit folgender Spezifikation verwendet:

- $92 \%$ MMA (Methylmetacrylat, monomer)

- 5 \% PMMA (Polymethylmetacrylat) mit einem Mol-gewicht von ca. 100000

- $3 \%$ BDAM (Butandiol-dimetacrylat)

- $2 \%$ Azeto-iso-buttersäurenitril (Härterkomponente)

- $2 \%$ Dibenzoylperoxid (Härterkomponente)

Durch Zugabe der beiden Härterkomponenten wurde die Polymerisationstemperatur von ursprünglich ca. $90^{\circ} \mathrm{C}$ auf ungefähr $60^{\circ} \mathrm{C}$ herabgesetzt. Diese relativ niedrige Temperatur wurde gewählt, um zum einen die thermische Beanspruchung des Materials möglichst gering zu halten und zum anderen würden höhere Temperaturen bei einer vor Ort Anwendung auch einen größeren technischen Aufwand erfordern. Eine weitere Herabsetzung der Polymerisationstemperatur ist zwar möglich erscheint jedoch nicht sinnvoll. Mit der weiteren Herabsetzung würde auch zwangsläufig die Glasübergangstemperatur, also der Übergang von 
mehr elastischem zu mehr plastischen Verhalten des Kunststoffs, weiter sinken und in den Schwankungsbereich der natürlichen Außentemperatur kommen.

\section{- Versuchsdurchführung}

Die Versuchdurchführung erfolgte in zwei Schritten. Im ersten Schritt wurden die intrinsischen Ultraschalleigenschaften der Marmorprobe charakterisiert. Die eigentliche Volltränkung erfolgte im zweiten Schritt. Dieser beginnt mit dem Anlegen eines Vakuums und anschließender Zufuhr der Tränkungslösung. Daraufhin wurde die imprägnierte Probe einer Wärmebehandlung unterzogen, um die Polymerisation einzuleiten. Der Tränkungsversuch wurde mittels Ultraschallmessungen diagnostisch begleitet.

Wie in der vorliegenden Arbeit ermittelt, sollten bei einer erfolgreichen Volltränkung die Mikrorisse des Marmors weitgehend geschlossen werden und die Ultraschallgeschwindigkeiten diejenigen eines rissfreien Gesteins erreichen. Um die Qualität der Volltränkung kontrollieren zu können, wurden daher zunächst die intrinsischen Ultraschallgeschwindigkeiten der Marmorprobe ermittelt. Eine Schließung der Mikrorisse wurde durch das Aufbringen eines Umschließungsdruckes bis $30 \mathrm{MPa}$ erreicht (s. Abb. 8.18). Die auf diese Weise ermittelten Kompressionswellengeschwindigkeiten zeigen ausgehend von 3,1 km/s einen Anstieg auf $6,1 \mathrm{~km} / \mathrm{s}$ bei einem Umschließungsdruck von $30 \mathrm{MPa}$. Für die Schwerwellengeschwindigkeiten ist eine Zunahme von $2,0 \mathrm{~km} / \mathrm{s}$ auf 3,3 km/s für die gleiche Belastung festzustellen.

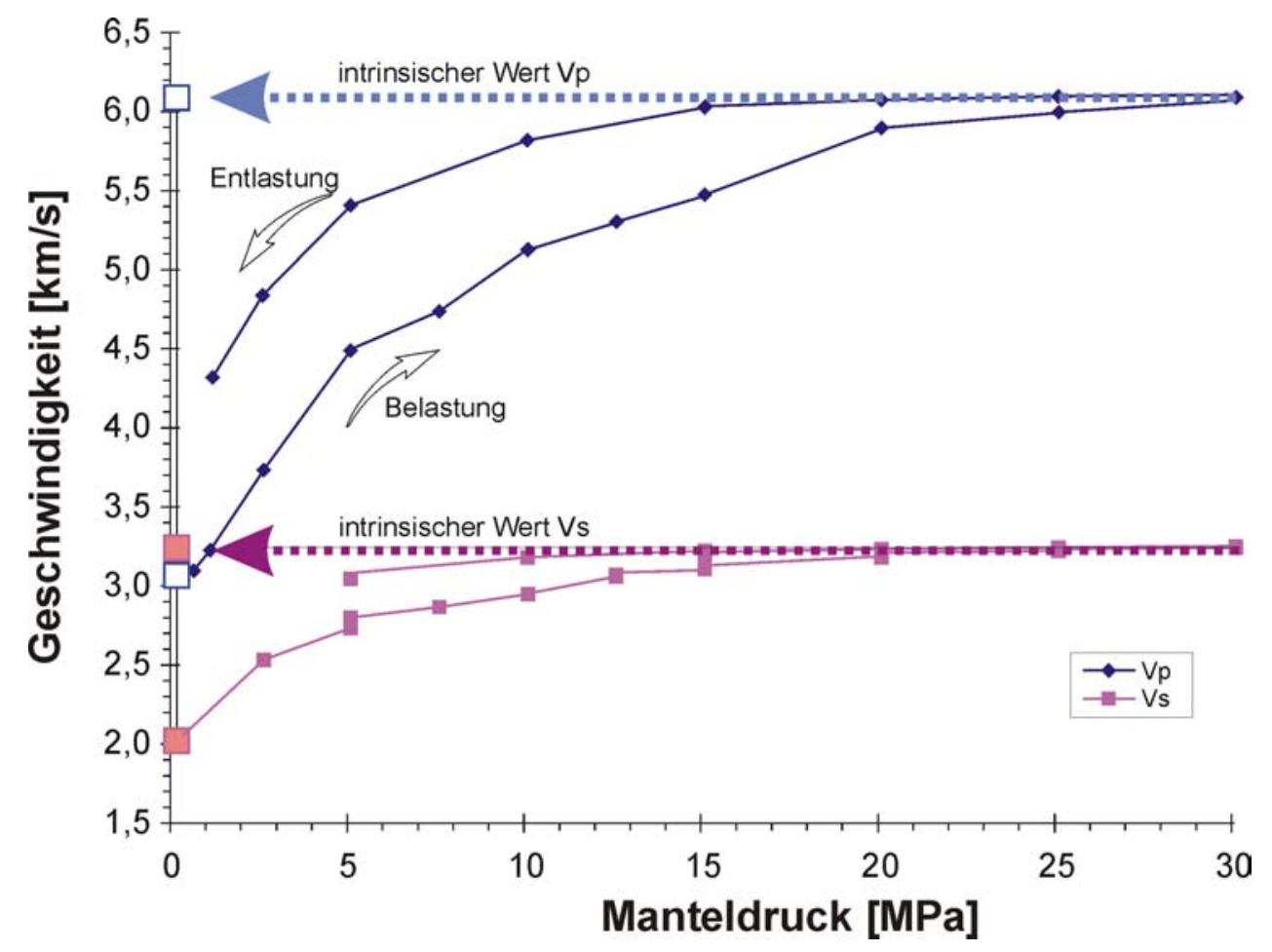

Abb. 8.18: Darstellung der Ultraschallgeschwindigkeiten als Funktion des Umschließungsdruckes bis $30 \mathrm{MPa}$ der thermisch geschädigten Probe aus Carrara Marmor.

Das Verhalten der Ultraschallgeschwindigkeiten während der Volltränkung ist in Abb. 8.19 dargestellt. Dabei liegen die Ausgangsgeschwindigkeiten bei $V_{p}=3,1 \mathrm{~km} / \mathrm{s}$ und 
$\mathrm{V}_{\mathrm{s}}=2,0 \mathrm{~km} / \mathrm{s}$. Das Anlegen des Vakuums erfolgte über einen Zeitraum von einer Stunde. Während dieser Versuchsphase sind keine Änderungen der Ausgangsgeschwindigkeiten festzustellen. Mit Beginn der Tränkung ist eine leichte Abnahme der Ultraschallgeschwindigkeiten $\mathrm{zu}$ ermitteln. Dieser Effekt wird bei der folgenden Temperaturerhöhung weiter verstärkt. Oberhalb einer Temperatur von ca. $45^{\circ} \mathrm{C}$ sind bei gleichbleibendem Stempeldruck in der Versuchsphase der Wärmebehandlung keine auswertbaren Ultraschallsignale empfangbar.

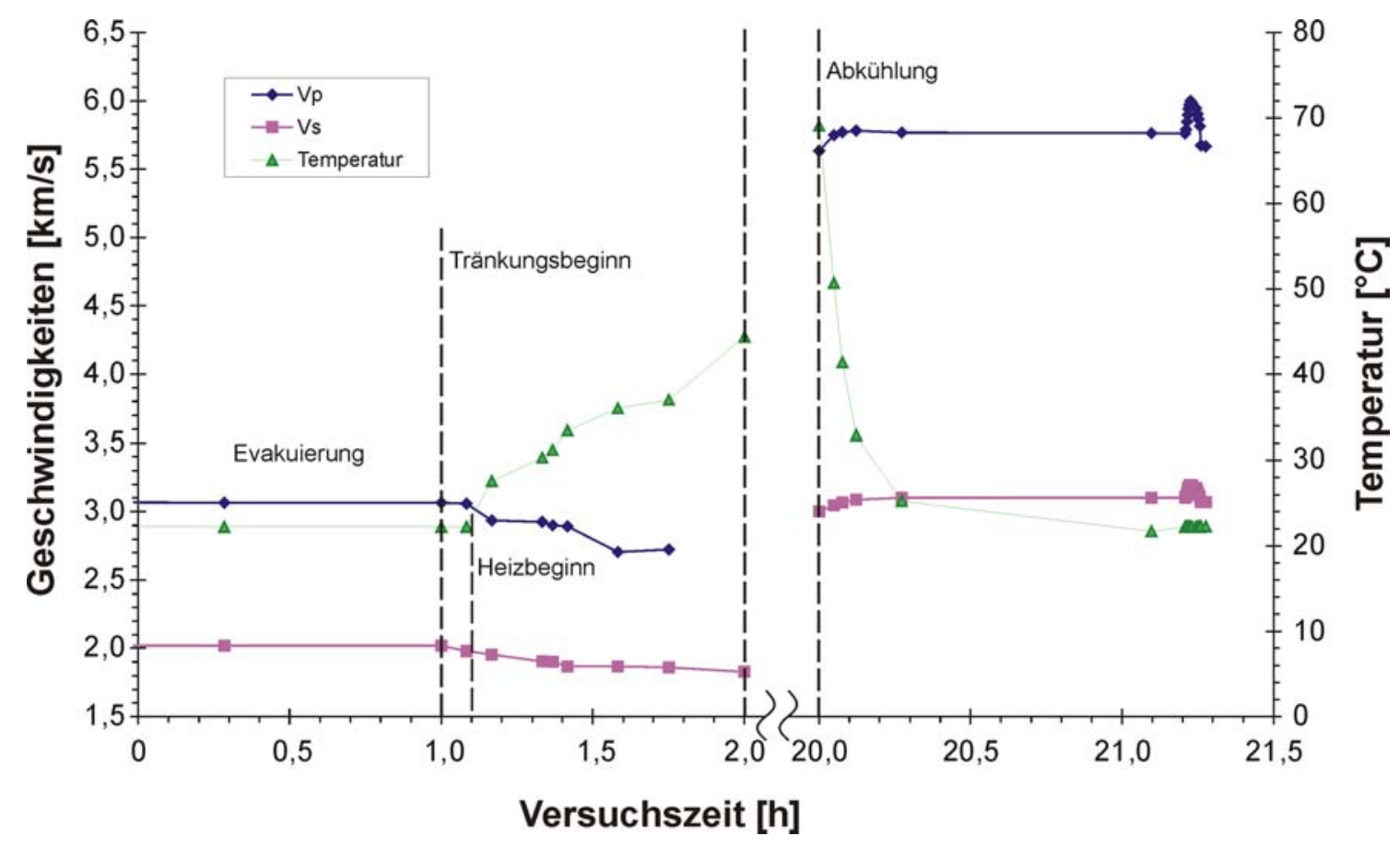

Abb. 8.19: Änderung der Ultraschallgeschwindigkeiten während der Versuchsdurchführung.

Beim vollständigen Erwärmen der imprägnierten Probe auf $70^{\circ} \mathrm{C}$ ist ein sprunghafter Anstieg der Ultraschallgeschwindigkeiten zu ermitteln. Dabei erreichen die Geschwindigkeiten mit $\mathrm{V}_{\mathrm{p}}=5,8 \mathrm{~km} / \mathrm{s}$ und $\mathrm{V}_{\mathrm{s}}=3,1 \mathrm{~km} / \mathrm{s}$ (s. Abb. 8.19) annähernd diejenigen des rissfreien Gesteins. Damit ist anhand der Ultraschalldaten festzustellen, dass die Mikrorisse weitgehend geschlossen und eine vollständige Imprägnierung der Probe mit dem vorgestellten Versuchsaufbau erreicht wurde. Bei Abkühlung der imprägnierten Probe ist keine Herabsetzung der Ultraschallgeschwindigkeiten zu beobachten.

\section{- Nachweis des Kunstharzes im Porenraum}

Nach REM-Untersuchungen an Bruchproben erscheint der Kunstharz als dünner, die Korngrenzen überziehender Film (Abb. 8.20). Dabei ist für die Mehrzahl der Korngrenzenflächen eine Dekorierung durch den Kunststofffilm festzustellen. Zum Teil fehlende PMMA-Überzüge sind auf das Abreißen von Filmen während der Bruchbildung, eine lokal unvollständige Tränkung oder nicht geöffnete Korngrenzen vor der Konservierungsmaßnahme zurückzuführen. 

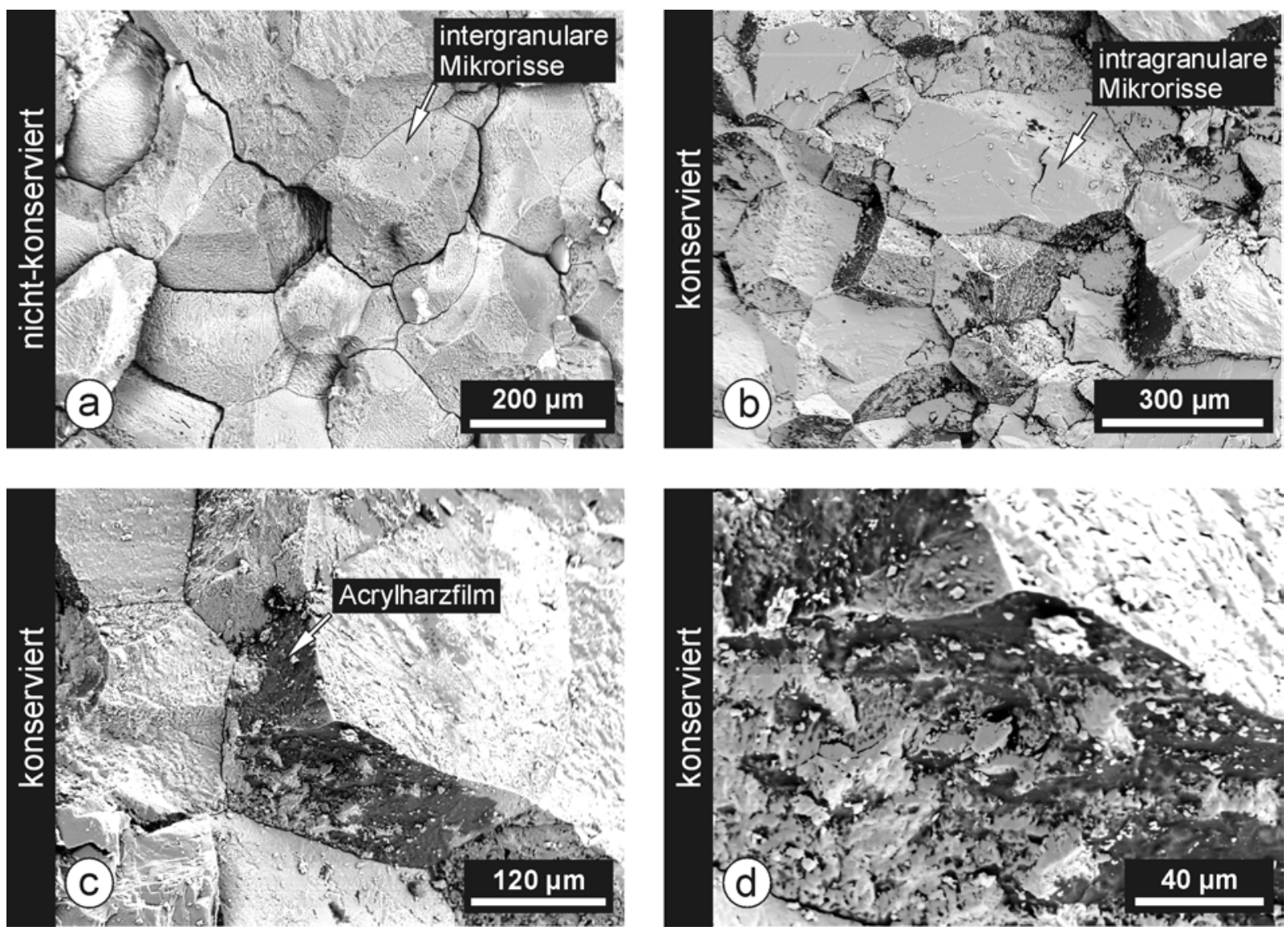

Abb. 8.20: REM-Aufnahmen (Überlagerung von Rückstreu- und Sekundärelektronenabbildung von Bruchproben) des untersuchten Carrara Marmors: a) vor der Konservierung mit vollständiger Entfestigung an Korngrenzen und b) nach der Konservierung mit häufiger Aktivierung von intragranularen Flächen als Ausdruck einer Gefügefestigung; und c,d) Detailaufnahmen des Acrylharzes auf Korngrenzenflächen.

Ein visueller Nachweis für eine ausgeprägte Festigung des Marmors, ist das Aufreißen unterschiedlicher Gesteinsdiskontinuitäten beim Brechen der Proben (vgl. Abb. 8.20a mit Abb. 8.20b). Hierbei weist die nicht-konservierte Probe eine reine Entfestigung an Korngrenzen auf. Hingegen zeigt die konservierte Marmorprobe eine häufige Aktivierung von intragranularen Flächen. Die Untersuchungen zeigen, dass der Kunststoff durch Verklebung zu einer Erhöhung der Kohäsion zwischen den Körnern führt und einem Aufbrechen an den Korngrenzen entgegenwirkt. Dies führt zum Entfestigen kristallinterner Diskontinuitäten, deren Aktivierung eine höhere Energie erfordert.

\section{- Änderung der Porenraumeigenschaften}

Die durchgeführte Volltränkung der Marmorprobe führt zu einer signifikanten Änderung der Porenraumeigenschaften. In Abb. 8.21a ist die Porosität und die Porengrößenverteilung des Ausgangszustandes der auf $200{ }^{\circ} \mathrm{C}$ vorkonditionierten Probe des Carrara Marmors dargestellt. Die mittels Quecksilberporosimetrie ermittelte Porosität beträgt hier $0,74 \%$. Die Porengrößenklassen weisen eine bimodale Verteilung auf, wobei sich das untere Maximum zwischen $0,0398 \mu \mathrm{m}$ und $0,0631 \mu \mathrm{m}$ und das obere zwischen $0,3981 \mu \mathrm{m}$ und $1,0000 \mu \mathrm{m}$ befindet. 
nicht-konserviert

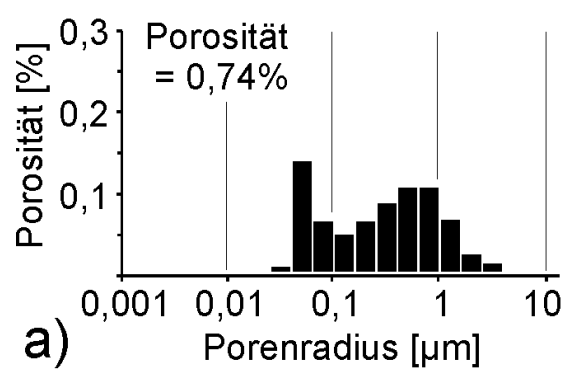

konserviert

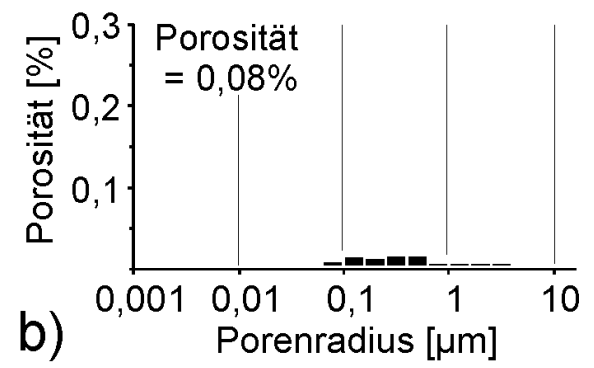

Abb. 8.21: Porenraumeigenschaften der untersuchten Probe aus Carrara Marmor a) vor und b) nach der modellhaften Volltränkung.

Für die konservierte Probe ist eine starke Herabsetzung der Porosität auf 0,08 \% zu ermitteln (s. Abb. 8.21). Damit wären ungefähr 90\% des ursprünglichen Porenraums verschlossen bzw. nicht mehr für das Quecksilber erreichbar. Die Porengrößenverteilung zeigt, dass alle Porenradienklassen fast vollständig reduziert sind. Damit zeigen auch die Analysen des Porenraums, dass die Poren weitgehend geschlossen wurden und somit eine vollständige Imprägnierung der Marmorprobe erfolgte. 


\section{Zusammenfassende Interpretation}

Ein wesentlicher Teil des kulturellen Erbes der Menschheit wird von Marmor gebildet. Insbesondere in den letzten Jahrzehnten wurde Marmor wieder zunehmend als Naturwerkstein, z.B. für großflächige Fassadenverkleidungen, eingesetzt. Mit dem verbreiteten Auftreten von starken Schädigungen hat die Verwitterungsproblematik von Marmor auch wirtschaftliche Bedeutung erlangt (GRIMM, 1999). Dabei erfahren der Witterung ausgesetzte Marmore durch physikalische, chemische und biologische Prozesse eine Gefügeauflockerung, die im Laufe der Zeit zur Zerstörung des Gesteins führt. Die Form und das Ausmaß der Verwitterung von Marmor ist neben der Exposition und den klimatischen Einflüssen auch wesentlich vom Gestein selbst abhängig. Die Hauptminerale eines kristallinen Marmors (Calcit und Dolomit) besitzen eine ausgeprägte Anisotropie der thermischen Ausdehnung. Dies kann bei entsprechenden klimatischen Verhältnissen ausschlaggebend für eine Dekohäsion der Körner und somit einer Gefügeauflockerung sein (GrimM, 1984; GRIMM \& SchwARZ, 1985). Dieser Prozess lässt sich mikroskopisch als Risswachstum an Korngrenzen und kristallinternen Flächen beschreiben. Mit der fortschreitenden Rissausbreitung erhöht sich die Porosität und bedingt dadurch eine verstärkte Einwirkung eindringender Niederschläge. Neben Frostsprengung treten chemische Auflösungserscheinungen und auch hygrische Dehnungsvorgänge auf (s. Diskussion in POSCHLOD, 1989; KÖHLER, 1991).

Die vorliegende Arbeit hatte zum Ziel, den Kenntnisstand über den bislang nur wenig untersuchten Einfluss des Gesteinsgefüges auf die thermomechanischen Entfestigungsprozesse von kristallinen Marmoren zu verbessern. Besondere Beachtung findet dabei die Frage, inwieweit die in Marmoren häufig entwickelten Gefügeanisotropien auch eine Richtungsabhängigkeit der Verwitterungsprozesse bedingen. Ein weiterer zentraler Punkt war die Charakterisierung des Einflusses von Konservierungen auf das Verwitterungsverhalten von Marmoren. Des Weiteren sollte geprüft werden, welchen Einfluss Gefügeregelungen auf die Beurteilung des Erhaltungszustandes mittels Ultraschall haben.

Das Grundkonzept der vorliegenden Arbeit basiert auf dem Vergleich von Marmoren in fünf verschiedenen Gesteinszuständen (bruchfrisch, natürlich verwittert, künstlich verwittert, modellhaft konserviert und konserviert/künstlich verwittert). Zur Charakterisierung der verschiedenen Materialzustände wurden verwitterungssensible petrophysikalische Parameter ermittelt (Porosität, Porengrößenverteilung, kapillare Wasseraufnahme, Ultraschallgeschwindigkeiten und Spaltzugfestigkeiten). Die künstliche Verwitterung erfolgte thermisch und wurde experimentell mittels Messungen der thermischen Dilatation beschrieben. Für die Konservierung wurden drei verschiedene Konservierungsverfahren angewandt: ein PMMA auf Lösungsmittelbasis $\left(\mathrm{PMMA}_{\mathrm{sol}}\right)$, ein vorkondensierter Polykieselsäureester (PKSE) und eine Acrylharzvolltränkung (PMMA poly). Für die Untersuchungen wurden Marmore aus Prieborn, Grosskunzendorf, Kauffung (Polen) sowie aus Carrara, Lasa, Sterzing (Italien) ausgewählt, welche sich durch eine große Variabilität von Gefügemerkmalen auszeichnen. 


\subsection{Gefügeabhängigkeit der thermomechanischen Entfestigungsprozesse von Marmoren}

Als entscheidender Prozess, der die Gefügeentfestigung in kristallinen Calcit-Marmoren einleitet, ist die thermisch gesteuerte Mikrorissbildung anzusehen. Dabei kommt den extrem anisotropen Einkristalleigenschaften von Calcit die entscheidende Bedeutung zu (EvANS, 1978; FREDRICH \& WONG, 1986). So variiert der Koeffizient der thermischen Dilatation $(\alpha)$ von Calcit nach KLEBER (1959) von positiven Werten $\left(\alpha_{11}=26 \cdot 10^{-6}{ }^{\circ} \mathrm{K}^{-1}\right)$ parallel zur kristallographischen c-Achse bis zu negativen Werten $\left(\alpha_{22}=\alpha_{33}=-6 \cdot 10^{-6}{ }^{\circ} \mathrm{K}^{-1}\right)$ parallel zu den a-Achsen. Durch diese Anisotropie der thermischen Dilatation können sich bei Temperaturwechseln Zug-, Druck- bzw. Scherspannungen im Gefüge aufbauen, die zum Aufreißen von Gesteinsdiskontinuitäten führen (BATTAGLIA et al., 1993; WIDHALM et al., 1996).

Neben der mineralogischen Zusammensetzung besitzt das Gefüge eine entscheidende Bedeutung für die Art und den Umfang der Gesteinsentfestigung bei thermomechanischer Beanspruchung. Dies beruht im Wesentlichen darauf, dass sich Mikrorisse generell entlang von Gefügeschwachstellen im Gestein entwickeln. Die in dieser Arbeit untersuchten sechs Marmorproben zeichnen sich durch eine große Vielfalt an Gefügeelementen aus (Textur, Kornformorientierung usw.). Die einzelnen Gefüge können zudem in ihrer Ausbildung stark variieren. Anhand der wesentlichen Gefügemerkmale können die Marmore in drei Korngefügetypen untergliedert werden. Typ I repräsentiert gleichkörnige Marmore mit polygonalen Kornformen (Carrara, Prieborn, Lasa). Das Korngefüge von Typ II ist durch eine ungleichmäßige (seriate) Korngrößenverteilung und durch eine deutliche Verzahnung der Körner gekennzeichnet (Grosskunzendorf, Sterzing). Typ III stellt einen Sonderfall dar, wobei es sich um ein heterogenes Korngefüge mit großen reliktischen Altkörnern, die von einer Matrix aus vorwiegend polygonalen Subkörnern und feinen rekristallisierten Körnern umgeben sind (Kauffung). Die bezüglich der Unterteilung herangezogenen Gefügemerkmale wie Korngrößenverteilung, Kornform und Korngrenzengeometrie haben signifikanten Einfluss auf die Entfestigung durch thermische Beanspruchung in der Art und Weise wie Mikrorisse aktiviert werden und somit auch auf die petophysikalischen Eigenschaften der unterschiedlichen Marmore.

\section{- Thermische Längenänderung}

Die anisotropen Dehnungseigenschaften des Calcit-Einkristalls führen in Verbindung mit den häufig in Marmoren vorkommenden Texturen zu einer Richtungsabhängigkeit der thermischen Dilatation. Je nach Intensität der Textur kann die Richtungsabhängigkeit schwach oder stark ausgebildet sein. Dabei können stark texturierte Marmore auch eine Kontraktion in bestimmte Gefügerichtungen aufweisen. Welche Richtung sich stärker als die andere dehnt wird durch das Muster der Textur bestimmt. Dabei können zwei Endglieder der Textur in kristallinen Marmoren definiert werden: der c-Achsen- und a-Achsenfasertyp.

Neben der Textur haben vorhandene und sich während der Beanspruchung neubildende Mikrorisse einen Einfluss auf die thermische Dehnung. Vorhandene offene Risse behindern die Übertragung der Einkristalleigenschaften auf das Gesamtgestein. So wird die Kontraktion 
parallel zur a-Achse des Calcit-Kristalls beim Vorhandensein eines entsprechend orientierten Mikrorisses nicht auf das Gestein übertragen, sondern der Riss wird sich weiter öffnen. Hingegen wird die starke Dehnung parallel zur c-Achse bei der Existenz eines penetrativen Risssystems sich deutlicher durchsetzen als in einem rissfreien Gestein. Die Neubildung von Mikrorissen bei einem Dehnungsereignis erhöht die Ausdehnung um den Betrag der Rissöffnung. Dabei kann sich der Beginn einer Rissneubildung bei sehr unterschiedlichen Temperaturen befinden und als kritischer Wert (,critical crack initiation temperature“: CCIT; vgl. RUEDRICH et al., 2001a) für die Sensibilität eines Marmors gegenüber thermischer Beanspruchung angesehen werden.

\section{- Thermisch bedingte Mikrorissbildung}

Die eigentliche Gefügeentfestigung von Marmoren erfolgt durch eine Mikrorissbildung, die zumindest initial weitgehend thermisch gesteuert ist und über Restdehnungen bei Dilatationsmessungen ermittelt werden kann. Die Ursache der thermischen Rissbildung sind die durch anisotrope Dehnungseigenschaften des Calcits hervorgerufenen Spannungen im Gestein. In welcher Art und in welchem Umfang eine Mikrorissbildung stattfindet, wird jedoch stark durch das Gesteinsgefüge des Marmors beeinflusst (vgl. SIEGESMUND et al., 2000c; ZEISIG et al., 2002). Generell werden bei Beanspruchung des Gesteins Gefügeschwachstellen aktiviert, wobei im Folgenden der Einfluss der wesentlichen Gefügemerkmale eines Marmors auf die verschiedenen Rissparameter (Rissart, Rissdichte, Rissregelung und Rissweite; s. Abb. 9.1) diskutiert werden soll.

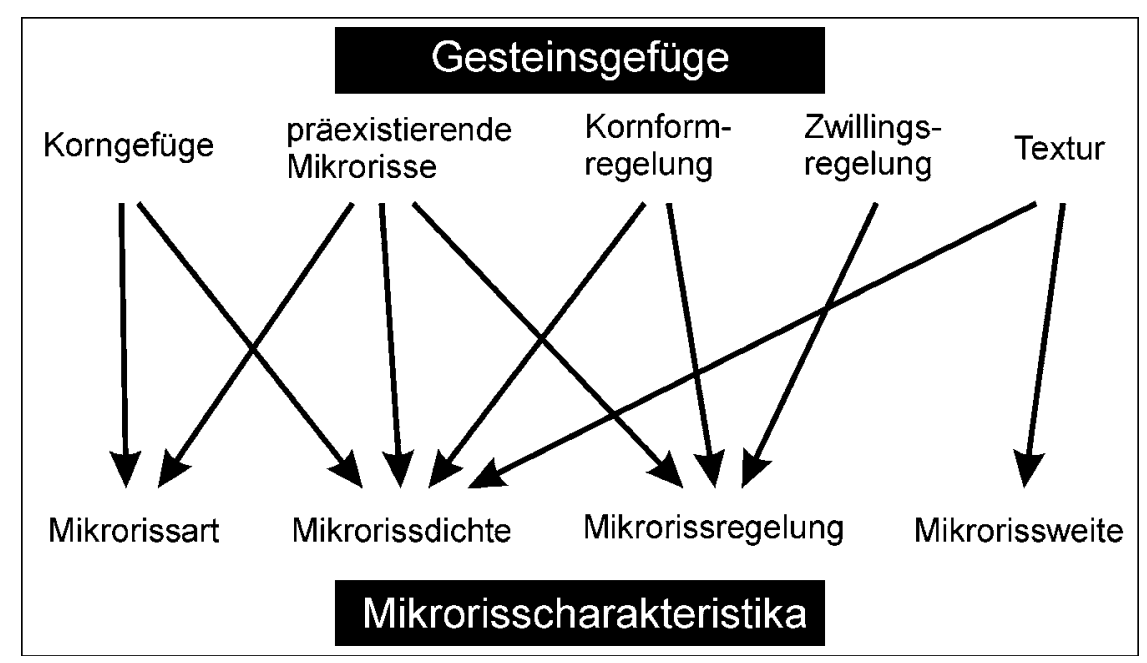

Abb. 9.1: Wesentliche Gesteinsgefüge von Calcit-Marmoren und ihr Einfluss auf verschiedene Mikrorisscharakteristika.

\section{Korngefüge}

Das Korngefüge wird durch die Korngrößenverteilung und die Korngrenzengeometrie bestimmt und besitzt entscheidenden Einfluss auf die Art der entstehenden Mikrorisse. Bei einem equigranularen polygonalen Korngefüge werden fast ausschließlich die Korngrenzen aktiviert. Hingegen zeigen seriate interlobate Korngefüge häufig eine Aktivierung von intragranularen Flächen wie Zwillings- und Spaltflächen. Für die Mikrorissdichte ist die 
Häufigkeit von Schwachstellen im Gestein verantwortlich. Für Marmore, die an Korngrenzen entfestigen, ist damit die Korngröße der entscheidende Parameter für die Mikrorissdichte.

\section{Präexistierende Mikrorisse}

Verheilte und mineralisierte präexistierende Mikrorisse repräsentieren weitere Schwachstellen des Gesteins, die bevorzugt durch mechanische Verwitterungsprozesse geöffnet werden. Dabei können Marmore, wie der in dieser Arbeit untersuchte Kauffunger Marmor, auch überwiegend entlang dieser Schwachstellen entfestigen. Hier hat die Häufigkeit und die bevorzugte Orientierung der präexistierenden Risssysteme den entscheidenden Einfluss auf die Rissdichte und die Rissregelung.

Kornformregelung

Die Kornformregelung bestimmt die bevorzugte Orientierung von Korngrenzen und ist somit bei Marmoren mit equigranularem polygonalem Korngefüge eine gerichtete Vorzeichnung von Schwachstellen. Diese können während der thermischen Beanspruchung aktiviert werden, womit sich ein gerichtetes Risssystem ausbildet.

\section{Zwillingsregelung}

Eine Zwillingsregelung hat eine ähnliche Bedeutung wie die Kornformregelung, jedoch vor allem für die ungleichkörnigen interlobaten Marmortypen. Bei diesen Marmoren kann durch eine ausgeprägte Textur lokal eine starke Regelung der Zwillinge auftreten und eine besondere Schwächung des Korngefüges darstellen. Hier kommt es häufig zu einer Ausbildung transgranularer Risse mit hohen Rissweiten, die offensichtlich besonders sensibel für spätere chemische Lösungsprozesse sind.

\section{$\underline{\text { Textur }}$}

Die Textur besitzt indirekt einen entscheidenden Einfluss auf die Rissweite, da die größten Rissweiten in die Richtung stärkster Dehnung beobachtet werden können. D.h., dass für die meisten Marmore die größten Rissweiten senkrecht zur bevorzugten Orientierung der cAchsen auftreten. Es ist davon auszugehen, dass in der Hauptdehnungsrichtung auch mehr Schwachstellen aktiviert werden und somit die Textur einen Einfluss auf die Mikrorissdichte besitzt.

\section{- Porenraum und Wasseraufnahmeverhalten}

Durch das Wachstum von Mikrorissen während thermischer Verwitterungsprozesse erhöht sich die Porosität und die Porengrößen ändern sich von kleinen zu großen. Im ,frischen“ Zustand weisen kristalline Marmore mit ca. 0,2\% eine sehr geringe Porosität auf. Mit zunehmender Verwitterung können Marmore auch höhere Porositäten erreichen. Mit bis zu $3 \%$ sind diese im Vergleich zu anderen Gesteinen aber immer noch sehr gering. Wesentliche Bedeutung für die fortschreitende Verwitterung von Marmoren besitzt vor allem die Veränderung der Rissweiten, welche hier als Porengrößen bezeichnet werden sollen und mittels quecksilberporosimetrischen Untersuchungen ermittelt wurden. Die Porengrößen frischer Marmore sind sehr gering und befinden sich weitgehend im Mikroporenbereich (unter $0,1 \mu \mathrm{m}$ ), womit der wesentliche Transportmechanismus des Wassers hier die Dampfdiffusion sein wird (SNeThlage, 1984). Mit zunehmender Verwitterung öffnen sich die Risse 
fortschreitend bis zu einigen $\mu \mathrm{m}$, womit sie zu Kapillarporen werden. Damit verbunden ist ein Übergang des Wassertransportmechanismus zu einem weitgehend kapillaren Transport von Wasser festzustellen, welcher wesentlich effizienter ist und zu einer starken Gefährdung von Marmoren hinsichtlich chemischer Lösungsprozesse führt.

Bei rein mechanischer Gefügeentfestigung ist von einem gleichbleibenden Verhältnis zwischen Porengrößenverteilung und Porosität für einen jeweiligen Marmor auszugehen, da ab einer bestimmten Entfestigung kaum neue Risse gebildet sondern vielmehr vorhandene Risse zunehmend geöffnet werden. Das Verhältnis ändert sich jedoch sobald andere Verwitterungsprozesse, wie vor allem die chemische Lösung einsetzen, die auf Korngrenzen und Spaltflächen zur Bildung selektiver Hohlräume führen. Dabei kann sich die Porosität deutlich erhöhen, wobei sich die Eintrittsgrößen für fluide Phasen nur bedingt ändern und somit die Porengrößen nur geringfügig zunehmen.

\section{- Ultraschallcharakteristik}

Aufgrund der Ausbildung hoher Mikrorissdichten in Verbindung mit einer starken Abnahme der Ultraschallgeschwindigkeiten während der Verwitterung von Marmoren, ist es möglich die Messung der Ultraschallgeschwindigkeiten als Beurteilungsverfahren bezüglich ihres Erhaltungszustandes einzusetzen. Für den Einsatz des Ultraschalls als diagnostisches Verfahren eignen sich vor allem die vor Ort leicht $\mathrm{zu}$ messenden Kompressionswellengeschwindigkeiten. Marmore besitzen im unverwitterten Zustand relativ hohe Kompressionswellengeschwindigkeiten von bis $\mathrm{zu} 7 \mathrm{~km} / \mathrm{s}$. Für einen weitgehend zerstörten Marmor, welcher eine vollständige Gefügeentfestigung aufweist und zumeist durch ein zuckerkörniges Absanden gekennzeichnet ist, kann hingegen die Geschwindigkeit bis auf $1 \mathrm{~km} / \mathrm{s}$ herabgesetzt sein. Die starke Reduktion beruht auf der hohen Effizienz von Mikrorissen bei der Herabsetzung der Ultraschallgeschwindigkeiten.

Für die Anwendung der Ultraschallgeschwindigkeitsmessungen als diagnostisches Verfahren ist es von ausschlaggebender Bedeutung, ob eine allgemein gültige Beziehung zwischen dem Verwitterungszustand und den Ultraschallgeschwindigkeiten von Marmoren besteht. KöHLER (1991) gründete auf umfangreichen $\mathrm{V}_{\mathrm{p}}$-Messungen an Carrara Marmoren eine Schadensklassifikation und definierte fünf Schadensklassen (s. Tab. 4.4). Die wesentlichen Einflussparameter für die Herabsetzung der Ultraschallgeschwindigkeiten sind die Rissart, die Rissdichten und die Rissweiten. Da diese Parameter in Marmoren sehr stark variieren können ist eine Korrelation nur bedingt möglich. Dabei zeigen die Marmore mit equigranularem polygonalem Korngefüge aus Carrara, Prieborn und Lasa eine gute Übereinstimmung der Schadensklassen mit den Ultraschalldaten. Dies ist darauf zurückzuführen, dass auch die Schadensklassifikation nach KÖHLER (1991) auf Ultraschalldaten gefügeähnlicher Marmore aus Carrara basiert. Die Gefügeähnlichkeit der equigranularen polygonalen Marmore besteht dabei hauptsächlich in der reinen, relativ gleichmäßigen und vollständigen Entfestigung entlang von Korngrenzen. Ungleichkörnige interlobate Marmore oder Sonderfälle wie der Kauffunger Marmor können häufig isolierte Risse und Risssysteme aufweisen, an denen das Gestein zerbricht. Aufgrund ausgeprägter Anisotropien dieser Risse sind sie jedoch nur in bestimmten Gefügerichtungen detektierbar oder besitzen nur geringe Auswirkung auf die Ultraschallgeschwindigkeiten. Eine universale Überführung der Schadensklassifikation auf 
alle Marmore erscheint daher nicht sinnvoll. Insbesondere bei Marmoren, die lokale und gerichtete Risssysteme ausbilden, ist die Übertragung nur bedingt, d.h. nur mit Erfassung ihrer Anisotropien möglich.

Von großer Bedeutung für die Ultraschalldiagnostik, insbesondere bezüglich von Untersuchungen direkt am Gebäude, ist der Einfluss des porenraumfüllenden Mediums auf die Kompressionswellengeschwindigkeiten. Die Füllung des Porenraums eines Marmors mit Wasser kann die Ultraschallgeschwindigkeiten deutlich erhöhen. Dabei weisen Marmore einer mittleren Verwitterung, welche im trockenen Zustand Kompressionswellengeschwindigkeiten um $4 \mathrm{~km} / \mathrm{s}$ besitzen, im wassergesättigten Zustand Werte eines frischen Marmors bis über $6 \mathrm{~km} / \mathrm{s}$ auf. Marmore die sehr stark verwittert sind und im trockenen Zustand nur um $2 \mathrm{~km} / \mathrm{s}$ zeigen, erreichen wassergesättigt nur Geschwindigkeiten zwischen 4 und $5 \mathrm{~km} / \mathrm{s}$. Da für Marmore, die im Außenbereich exponiert sind, generell von einer bestimmten Menge Wasser im Porenraum auszugehen ist, sollte dies bei der Anwendung vor Ort, Berücksichtigung finden. Eine eindeutige Charakterisierung und Einordnung in bestimmte Schadensklassen kann i.d.R. nur mit ergänzenden Laboruntersuchungen erfolgen.

\section{- Gefügeabhängigkeit der Zugfestigkeiten von Marmoren}

Kristalline Marmore weisen schon im unverwitterten Zustand, im Vergleich zu anderen kristallinen Gesteinen, relativ geringe Zugfestigkeiten auf (vgl. FLEISCHER, 2002 und STROHMEYER \& SiEgESMUND, 2002). Neben dem allgemeinen Einfluss der mineralogischen Zusammensetzung ist die Festigkeit bei Zugbeanspruchung aber vor allem vom Gefügeinventar abhängig (BROSCH et al., 2000). Dies ist darauf zurückzuführen, dass ein erzwungener Zugbruch bei mechanischer Beanspruchung bevorzugt entlang von Schwächezonen des Gefüges verläuft. Dabei schwächen insbesondere vorhandene offene Mikrorisse den Gesteinskörper, da an ihnen Spannungsspitzen auftreten und sich der Bruch ausbreitet (PECK et al., 1985). Für kristalline Marmore sind aber auch weitere Gefügeelemente wie die Korngröße, die Kornverzahnung und die Textur wesentliche Einflussparameter, die das mechanische Verhalten bei Zugbeanspruchung beeinflussen. Da die Gefügeparameter häufig Anisotropien ausbilden, besitzen Marmore zumeist auch eine deutliche Richtungsabhängigkeit der Zugfestigkeiten.

Der Zugbruch in Marmoren mit equigranularem polygonalem Korngefüge wie beim Carrara Marmor, entwickelt sich beim Spaltzugversuch fast ausschließlich an Korngrenzen. Für diese Marmortypen können starke Zugfestigkeitsanisotropien vor allem durch eine Kornformorientierung hervorgerufen werden. Für ungleichkörnige Marmore mit verzahnten Korngrenzen wie dem Sterzinger Marmor, ist hingegen der Zugbruch überwiegend entlang intragranularer Flächen entwickelt. Hier ist vor allem eine ausgeprägte Textur in Verbindung mit einer Regelung von Zwillings- und Spaltflächen für die starke Anisotropie der Zugfestigkeiten verantwortlich.

\subsection{Auswirkungen von Konservierungen auf die Marmoreigenschaften}

Seit langem wird versucht mittels Konservierungen die Festigkeit von Marmoren zu erhöhen und ihre Resistenz gegenüber der Verwitterung zu verbessern. Um zu überprüfen, welche 
Auswirkungen eine Konservierung auf das Materialverhalten von Marmoren besitzt, wurden in der vorliegenden Arbeit drei Konservierungsverfahren angewandt. Dazu wurde ein Polymethylmetacrylat auf Lösungsmittelbasis $\left(\mathrm{PMMA}_{\mathrm{sol}}\right)$, ein Polykieselsäureester in gelöster Form (PKSE) und eine Volltränkung mit Acrylharz $\left(\mathrm{PMMA}_{\text {poly }}\right)$ durchgeführt. Diese drei Verfahren repräsentieren dabei unterschiedliche Wirkprinzipien. Bei dem $\mathrm{PMMA}_{\mathrm{sol}}{ }^{-}$ Verfahren handelt es sich um die Abscheidung eines Kunstharzfilms, welcher lokal die Körner überzieht und klebende Wirkung besitzt. Im Gegensatz dazu repräsentiert das PKSEVerfahren die Abscheidung eines mineralischen Bindemittels, also die lokale Ausfällung einer stützenden Substanz. Das dritte Wirkprinzip der Volltränkung (PMMA poly) führt zu einer weitgehenden Ausfüllung der Poren und verhindert den Eintritt von flüssigen Phasen in das Gestein.

\section{- Änderung der Porenraumeigenschaften}

Das Einbringen von Konservierungsmitteln in den Porenraum eines Gesteins ändert zwangsläufig seine Porenraumeigenschaften. Hierbei führen die verschiedenen Konservierungsmethoden zu einer verfahrensspezifischen Veränderung des Porenraums. Das

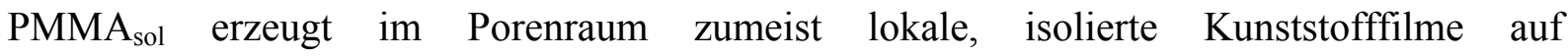
Kristalloberflächen. Hingegen ist das Vorkommen des PKSE im Porenraum durch ein lokales Auftreten scherbenartiger Fragmente gekennzeichnet. Sowohl das PMMA poly- als auch das PKSE-Verfahren bewirken, aufgrund relativ hoher Wirkstoffgehalte (ca. $40 \%$ ), eine Verminderung der Porosität um ca. 1/3. Die häufigst besetzte Porenradienklasse wird nach unten gesetzt, wobei kleinere Poren noch vorhanden sind. Dies bedeutet, dass bevorzugt größere Poren durch die Konservierungen ausgefüllt wurden. Für die konservierten Proben bleibt dabei ein kapillarer Wassertransport erhalten.

Das $\mathrm{PMMA}_{\text {poly }}$-Verfahren füllt hingegen die Porenräume weitgehend aus. Ein sekundärer Porenraum wird durch Vakuolen gebildet, welche durch die Volumenreduktion während der Polymerisation entstanden sind. Dieser Porenraum ist nicht mehr für flüssige Phasen zugänglich. Resultierend besitzen die vollimprägnierten Marmore praktisch keine effektive Porosität und keinen kapillaren Wassertransport.

\section{- Änderung petrophysikalischer Parameter durch die Konservierung}

Für alle Konservierungsverfahren ist eine Erhöhung der Kompressionswellengeschwindigkeiten festzustellen. Die zu erreichenden Geschwindigkeiten sind jedoch verfahrensspezifisch. So ist hier für die $\mathrm{PMMA}_{\text {sol }}$ konservierten Marmore eine deutliche und für die PKSE konservierten eine nur schwache Erhöhung der Ultraschallgeschwindigkeiten festzustellen. Letzterer Sachverhalt gilt besonders für stark verwitterte Proben. Die mittels Acrylharzvolltränkung konsolidierten Marmore erreichen hingegen sehr hohe Kompressionswellengeschwindigkeiten um 6 km/s. Die Geschwindigkeiten liegen im Bereich der intrinsisch zu erwartenden, was eine weitreichende Schließung der Risse anzeigt. Hierbei erreichen auch stark entfestigte Marmore, die zuvor Kompressionswellengeschwindigkeiten von $2 \mathrm{~km} / \mathrm{s}$ aufzeigten, im konservierten Zustand Geschwindigkeiten um $6 \mathrm{~km} / \mathrm{s}$. 
Die Spaltzugfestigkeiten werden durch alle Konservierungsverfahren erhöht, wobei der Anstieg jedoch sehr stark vom angewandten Wirkprinzip abhängig ist. Die Anwendung des $\mathrm{PMMA}_{\text {sol }}$ Verfahrens, welches zu einer lokalen Verklebung der Körner führt, weist eine mittlere Erhöhung der Zugfestigkeiten auf. Der Polykieselsäureester führt zu einer Stabilisierung des Korngerüsts, trägt jedoch, aufgrund seiner begrenzten Haftung an den Calcitoberflächen, nur in begrenztem Maße zur Erhöhung der Zugfestigkeiten bei. Hingegen erreichen die mittels PMMA poly-Verfahren konservierten Marmore sehr hohe Spaltzugfestigkeiten um $8 \mathrm{MPa}$. Auffällig ist jedoch für die Korngefügetypen I und II ein sehr unterschiedliches Verhalten der Richtungsabhängigkeit der Zugfestigkeiten. Für den Carrara Marmor, welcher durch eine reine Korngrenzenentfestigung gekennzeichnet ist, führt die Konservierung zu einer starken Reduktion der Zugfestigkeitsanisotropien. Der Sterzinger Marmor hingegen behält seine starke Richtungsabhängigkeit.

\section{- Dehnungsverhalten nach einer Konservierungsmaßnahme}

Die in der vorliegenden Arbeit durchgeführten Konservierungen ändern signifikant das Dehnungsverhalten der Marmore. Für die verwendeten Acrylharze ist dabei ein starker Einfluss der jeweiligen Glasübergangstemperaturen auf das Dehnungsverhalten festzustellen. Weiterhin ist die Bindung zwischen Konservierungsmittel und den Calcit-Kristallen von entscheidender Bedeutung. So ist für das PKSE-Verfahren, welches keine Verklebung erzeugt, nur eine Stabilisierung des Korngerüstes festzustellen. Die weitreichende Wiederherstellung der Kohäsion der Körner durch die Acrylharzvolltränkung führt hingegen zu einer starken Abnahme der absoluten Dehnung der Marmore.

$\underline{\text { Einfluss der Glasübergangstemperatur auf das Dehnungsverhalten }}$

Für die mittels Acrylharz konservierten Marmorproben kann ab einer bestimmten Temperatur eine sprunghafte Zunahme der Dehnung und damit verbunden eine ausgeprägte Restdehnung ermittelt werden. Dieser Effekt ist auf die Überschreitung der jeweiligen Glasübergangstemperatur zurückzuführen. Für die $\mathrm{PMMA}_{\text {sol }}$ konservierten Proben ist dies bei erreichen von ca. $55{ }^{\circ} \mathrm{C}$ gegeben, wohingegen die $\mathrm{PMMA}_{\text {poly }}$ konservierten Proben erst bei ca. $80{ }^{\circ} \mathrm{C}$ diesen Dehnungssprung aufweisen. Die Glasübergangstemperatur repräsentiert dabei den Temperaturbereich, bei dem der Kunststoff vom mehr elastischen in den mehr plastischen Zustand übergeht. Die Erweichung resultiert in einer ausgeprägten Restdehnung, wobei im Gegensatz zum normalen Dehnungsverhalten hier kaum Risswachstum stattfindet, also die Bildung neuer Risse bzw. das Abreißen von Kunststofffilmen. Die weitgehend durch die plastische Verformung des Kunststoffes hervorgerufenen Restdehnungen können durch eine nur sehr geringe Herabsetzung der Ultraschallgeschwindigkeiten nach thermischer Beanspruchung nachgewiesen werden.

\section{Stabilisierung des Korngefüges durch PKSE}

Die mittels PKSE konsolidierten Marmorproben weisen zumeist eine normales Dehnungsverhalten auf, welches vergleichbar zu einem nicht verwitterten Marmor ist. Dabei kann in der Mehrzahl der Fälle auch eine deutliche Restdehnung nach thermischer Beanspruchung festgestellt werden, welche auf eine fortschreitende Rissweitung 
zurückzuführen ist. Die fortschreitende Entfestigung geht dabei einher mit einer ausgeprägten Abnahme der Ultraschallgeschwindigkeiten.

Bei stark verwitterten Proben kann das Kieselgel zu einer deutlichen Stabilisierung des Korngerüstes führen. Für eine stark verwitterte Carrara Marmorproben kann beispielsweise ein Gefügekollaps, also eine fortschreitende Kontraktion bei thermischer Erwärmung festgestellt werden, die nicht auf die Kristalleigenschaften sondern auf die starke Auflockerung der Probe zurückgeführt werden kann. Nach der Konservierung mit PKSE ist diese Kontraktion nicht mehr zu beobachten und es kommt zu einer Dehnung, vergleichbar zu einer frischen Probe.

Herabsetzung der Dehnung durch eine Volltränkung

Die Volltränkung mittels $\mathrm{PMMA}_{\text {poly }}$ eines Marmors führt zu einer deutlichen Herabsetzung der absoluten Dehnung bei thermischer Beanspruchung. Dies erscheint aufgrund des höheren Ausdehnungskoeffizienten des PMMAs $\left(\alpha=70 \cdot 10^{-6} \mathrm{~K}^{-1}\right)$ gegenüber dem des CalcitEinkristalls (Volumenausdehnungskoeffizient von ca. $\alpha=13 \cdot 10^{-6} \mathrm{~K}^{-1}$ ) erst einmal widersprüchlich. Es wäre zu erwarten, dass beim Zusammenführen zweier Materialien der Gesamtausdehnungskoeffizient die Summe aus den Einzelkoeffizienten der jeweiligen Materialien ist. Der wesentliche Grund für die Herabsetzung der Dehnung ist die verbesserte Übertragung der Einkristalleigenschaften auf das Gesamtgestein. Ein verwitterter Marmor weist eine gesteinsdurchdringende Entfestigung an Mikrorissen auf, die das Dehnungsverhalten puffern. So kann vor allem die Kontraktion parallel zur a-Achse des Calcit-Kristalls bei einem penetrativ durch Mikrorisse geschädigten Marmor nicht mehr auf das Gesamtgestein übertragen werden. Durch die Volltränkung werden die einzelnen Körner verklebt, so dass auch die Kontraktion des Calcit-Kristalls parallel zur a-Achse wieder auf das Gestein übertragen werden kann. Die Veränderung des Dehnungsverhaltens eines Marmors nach einer Volltränkungsmaßnahme kann auch von praktischer Relevanz sein. Dies gilt besonders für stark texturierte Marmore, welche im vollimprägnierten Zustand auch eine deutliche Kontraktion in bestimmte Gefügerichtungen aufweisen können. Dabei können deutliche Dehnungskontraste zu nicht konservierten Bereichen des Gesteins aber auch zu anderen Materialien entstehen.

\subsection{Bedeutung der gefügeabhängigen Marmorverwitterung für die vor Ort Problematik}

Um die Bedeutung der gewonnenen Erkenntnisse über die Gefügeabhängigkeit der Marmorverwitterung sowie der Konservierung bezüglich der vor Ort Problematik zu überprüfen, erfolgten in der vorliegenden Arbeit vergleichende Untersuchungen in Form von Fallbeispielen. Als Modellfall wurde das Marmorpalais in Potsdam ausgewählt. Die hier verbauten Marmore weisen eine große Vielfalt an Gefügemerkmalen auf. In einem einfachen Fallbeispiel wurde die Gefügeabhängigkeit der Schadensformen von Marmoren geprüft. Anhand eines weiteren Fallbeispiels erfolgte eine Charakterisierung des Einflusses von Gefügeanisotropien auf die tomographische Auswertung von Ultraschalldaten. Weiterhin wurde experimentell die Möglichkeit der Durchführbarkeit einer Volltränkung vor Ort überprüft. 


\section{- Gefügeabhängige Schadensphänomene}

Die am Marmorpalais in Potsdam ursprünglich verbauten Marmore aus Carrara, Prieborn, Grosskunzendorf und Kauffung sind seit ca. 200 Jahren der Bewitterung ausgesetzt. Die untersuchten Gesteine weisen sehr vielfältige Schadensmerkmale auf. Obwohl die Marmorobjekte sehr unterschiedlich am Gebäude exponiert sind und lokal die klimatischen Bedingungen deutlich variieren, sind die Hauptschadensformen der untersuchten Marmore individueller Art und können auf bestimmte Gefügemerkmale zurückgeführt werden. Die jeweiligen Schadensphänomene korrelieren dabei mit den in dieser Arbeit untergliederten Korngefügetypen.

Die wesentliche Schadensform der Marmore mit equigranularem polygonalem Korngefüge aus Carrara und Prieborn ist die deutliche Aufrauung der Oberflächen, welche im fortgeschrittenen Verwitterungszustand zum sandigen Abzuckern der oberen Gesteinspartien führt. Dies ist auf die reine Korngrenzenentfestigung und die damit verbundene schwache Einbindung der polygonalen Körner in das Korngefüge dieser Marmore zurückzuführen.

Für den Grosskunzendorfer Marmor mit seriatem interlobatem Korngefüge ist ebenfalls eine deutliche Reliefbildung an der Oberfläche zu beobachten. Ein Absanden tritt jedoch seltener sowie in schwächerer Intensität auf und ist zumeist an Bearbeitungskanten gebunden. Dies ist sicherlich auf die stärkere Verzahnung der Körner zurückzuführen. Weiteres Schadensmerkmal dieses Marmors sind bevorzugt entlang von Spaltflächen und Zwillingsebenen verlaufende transgranulare Risse, welche aus der deutlichen Regelung dieser Flächen bedingt durch die ausgeprägte Texturierung des Marmors resultieren.

Der Kauffunger Marmor, der bezüglich seines Gefüges eine Sonderstellung einnimmt, entfestigt überwiegend an präexistierenden mineralisierten und verheilten Rissen. Die Gefügematrix weist hingegen kaum eine Entfestigung auf, womit auch die chemische Rückwitterung der Oberflächen aufgrund der begrenzten Angriffsfläche nur schwach ausfällt.

\section{- Einfluss von Gefügeanisotropien auf die Zustandsdiagnostik mittels Ultraschall}

Für Marmore, die eine gesteindurchdringende Entfestigung entlang von Mikrorissen aufweisen, ist die Kenntnis des Erhaltungszustandes des unzugänglichen Inneren von besonderer Bedeutung. In der Praxis wurden hierfür in den letzten Jahrzehnten zunehmend ultraschalltomographische Untersuchungen eingesetzt. Um zu überprüfen inwieweit die in Marmoren vorhandenen Gefügeanisotropien die tatsächliche Ultraschallgeschwindigkeitsverteilung überlagern, wurden in der vorliegenden Arbeit ultraschalltomographische Untersuchungen an zwei Säulen aus Prieborner und Kauffunger Marmor des Marmorpalais in Potsdam durchgeführt. Zusätzlich wurden alle relevanten Gefügeelemente der Säulen (Makrorisse, Foliation usw.) mittels Detailkartierungen erfasst.

Im Fall der Prieborner Marmorsäule weisen die Gefügekartierungen ein steil stehendes Risssystem aus, welches als wesentliche Schwächung der Säule anzunehmen ist. Diese makroskopisch ermittelbaren Risssysteme bilden sich in den in mehreren Ebenen ermittelten Ultraschalltomogrammen nicht $\mathrm{ab}$. Die Tomogramme zeigen vielmehr eine lokale leicht 
dezentrierte Kernentfestigung an und stehen somit nicht im Einklang mit den Detailkartierungen. Aus Laboruntersuchungen ist bekannt, dass der Prieborner Marmor eine starke Ultraschallgeschwindigkeitsanisotropie aufweist, welche durch eine ausgeprägte Textur und durch orientierte Mikrorisse hervorgerufen wird. Um zu überprüfen, inwieweit diese starke Anisotropie das Muster eines Tomogramms verändert, wurde aus den Labordaten ein synthetisches Tomogramm errechnet. Hierzu wurden Ultraschalldaten verwendet, die einer bestimmten Ebene aus Kugelmessungen, vergleichbar den Messungen an der Säule, entnommen wurden. Das Ergebnis zeigt, dass allein durch die Textur und die orientierten Mikrorisse im Gestein ein ausgeprägtes Verteilungsmuster in einem Tomogramm hervorrufen werden kann. Basierend auf dieser Kenntnis wurden verschiedene, entsprechend ausgerichtete, Anisotropieverhältnisse in die Säulentomogramme eingerechnet. Dabei wird aus der Kernentfestigung in den Tomogrammen, mit zunehmender Anisotropie, ein die Säule durchziehender Niedriggeschwindigkeitskanal. Dieser korreliert mit den makroskopisch ermittelbaren Risssystemen, womit die Tomogramme mit den Detailkartierungen im Einklang stehen. Die Ergebnisse zeigen somit, dass die in Marmoren häufig ausgeprägten Ultraschallanisotropien lokale Entfestigungen wie Makrorisse oder auch Randentfestigungen überprägen, die bei Nichtbeachtung zu Fehlinterpretationen führen können.

Im Fall des untersuchten Kauffunger Marmors fokussierten die ultraschalltomographischen Untersuchungen auf den Einfluss der Risssysteme in den verschiedenen Skalenbereichen. Dabei stellt der Kauffunger Marmor einen Sonderfall dar, der nur untergeordnet gesteinsdurchdringend an Mikrorissen verwittert sondern vor allem eine Entfestigung entlang von verheilten und mineralisierten/versiegelten makroskopisch erkennbaren Risssystemen aufweist. Aus den Säulenkartierungen konnten drei Makrorisssysteme ermittelt und quantifiziert werden. Eine bevorzugte Orientierung der Mikrorisssysteme, die auch in einer Herabsetzung der Ultraschallgeschwindigkeiten resultieren sollte, wird durch eine intrinsische Anisotropie von Kompressionswellengeschwindigkeiten, ermittelt aus Kugeldaten, verstärkt. Beim Vergleich der ermittelten Risse mit den Tomogrammen ergibt sich eine gute Übereinstimmung.

Abschließend kann festgestellt werden, dass Ultraschallanisotropien einen deutlichen Einfluss auf eine tomographische Inversion von Ultraschalldaten besitzen können. Detaillierte Gefügekartierungen können dabei nicht nur wesentliche Informationen über die strukturellen Schwachstellen des Gesteins liefern, sondern auch bei der Interpretation von Ultraschalldaten nützlich sein.

\section{- Möglichkeiten einer Volltränkung von Marmorobjekten vor Ort}

Die Acrylharzvolltränkung wird seit längerem für die Konservierung von Natursteinen eingesetzt. Da dieses Konservierungsverfahren nicht vor Ort angewandt wird, müssen die zu tränkenden Objekte ausgebaut und zur Tränkungsanlage transportiert werden. Für einige Objekte, welche nur schwer aus Gebäuden entfernt werden können, stellt sich daher die Frage ob eine entsprechende Volltränkung von Marmor auch direkt am Objekt durchgeführt werden kann. Um dies zu prüfen wurde eine Acrylharzvolltränkung im Labormaßstab durchgeführt. Die Volltränkung erfolgte an einer zylindrischen Marmorprobe, die in eine Gesteinspresse 
einaxial eingespannt wurde. Bei dem Gesteinszylinder handelt es sich um eine thermisch aufgelockerte Carrara Marmorprobe mit typisch equigranularem polygonalem Korngefüge.

Sowohl ein starker Anstieg der Ultraschallgeschwindigkeiten von $V_{p}=3 \mathrm{~km} / \mathrm{s}$ auf $\mathrm{V}_{\mathrm{p}}=6 \mathrm{~km} / \mathrm{s}$ als auch die fast vollständige Reduktion der erreichbaren Porosität belegen die erfolgreiche Volltränkung. Das nur lokale Auftreten des Kunstharzes im Porenraum sowie die häufige Aktivierung von intragranularen Kristallflächen von Bruchproben zeigen eine starke Verklebung der einzelnen Körner an. Da das monomere Acrylharz sehr geringe Molekülgrößen aufweist und verwitterte Marmore fast immer eine gesteinsdurchdringende gut vernetzte Rissporosität besitzen, kann auch von einer Durchführbarkeit der Volltränkung größerer Marmorobjekte vor Ort ausgegangen werden. Dies gilt insbesondere für Marmore mit equigranularem polygonalem Korngefüge, die eine weit reichende Korngrenzenentfestigung aufweisen.

\section{4 Überführung der Erkenntnisse in den Umgang mit Marmor in der Praxis}

Basierend auf den in dieser Arbeit gewonnenen Erkenntnissen und Erfahrungen kann ein Konzept zur materialspezifischen Charakterisierung von Marmorobjekten aufgestellt werden (Abb. 9.2). Diese vereinfachte Vorgehensweise konzentriert sich dabei auf wesentliche Fragen des Erhaltungszustandes und der gefügebedingten Entfestigungsmechanismen. Da im Einzelfall jedes Marmorobjekt seine eigenen Charakteristika aufweist (z.B. Salzbelastung, starke Besiedlung durch Organismen, etc.), muss je nach Situation das Konzept objekt- und materialspezifisch angepasst und oder ergänzt werden.

Für zahlreiche Fragestellungen ist dabei zu Anfang die Objektsituation aufzunehmen (Modul A). Dabei können trivial erscheinende Informationen wie Expositionsdauer und Ursprungszustand wichtige Daten zum Materialverlust liefern. Von entscheidender Bedeutung ist auch die Feststellung des klimatischen Umfeldes. Da Marmore gesteinsdurchdringend entfestigen, beinhaltet die Untersuchung des Objektes vor Ort, neben der Erfassung der Oberflächenschädigungen, vor allem auch die Charakterisierung des unzugänglichen Inneren mit vorzugsweise indirekten Methoden (Modul B). Für die detaillierte Erfassung eines Objektes aus Marmor ist vor allem eine genaue Aufnahme der Gesteinsstrukturen (Foliation, Makrorisse etc.), bevorzugt mittels maßstabsgetreuer Gefügekartierung sinnvoll. Dies ermöglicht eine genaue Korrelation mit den geschädigten Bereichen in einer Schadenskartierung. Bezüglich der Erfassung der Innenverwitterung eignen sich besonders indirekte Messungen mittels Ultraschall. Für die Ermittlung der Entfestigung in oberflächennahen Bereichen ist auch der Einsatz direkter Verfahren, wie der Messung des Festigkeitsprofils mittels Bohrwiderstand möglich. Zusätzlich können weitere Untersuchungen erforderlich sein, wie die Beschreibung des Wasseraufnahmeverhaltens hinsichtlich zu erwartender Konservierungsmaßnahmen oder Verformungsmessungen, z.B. an Fassadenplatten. 


\section{Modul A: Objektsituation}

Marmormaterialien

Aufnahme und Unterscheidung der verwendeten Materialien (auch Ergänzungen) im Einzelfall mittels Objektkartierung

\section{Ursprungszustand}

Feststellung der ursprünglichen Geometrie

Sanierungen, vorherige

Klärung vormaliger Schutz und Erhaltungsmaßnahmen (z.B.

Reinigungen, Konservierungen)

\section{Klimabedingungen und Exposition}

Ermittlung der allgemeinen und lokal am Objekt herschenden Klimabedingungen sowie weitere Informationen wie z.

\section{$\underline{\text { Randparameter }}$}

Reststellung von einflußnehmenden Randparametern wie z.B.

Überdachungsschäde
Modul B: Untersuchungen vor Ort

Schadenserfassung der Oberfläche

Qualitative und Quantitative Aufnahme der äußeren

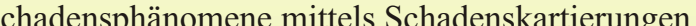

\section{Makrogefügeaufnahme}

detaillierte Gesteinsbeschreibung mit maßstabsgetreuer Aufnahme in Form von Gefügekartierungen

Schadensaufnahme des Inneren

Ermittlung des Erhaltungszustandes im unzugänglichen Inneren z.B. mittels Ultraschallanalyse (im Einzelfall auch Ultraschall-

tomographie)

Schadensprofil

Aufnahme des Festigkeitsprofils z.B. mittels Mikrobohrhärte

\section{Wasseraufnahme Verhalten}

Charakterisierung des Wasseraufnahmeverhaltens bei stark geschädigten Objekten (z.B. nach Karsten).

\section{Modul C: Materialcharakterisierung}

Mikrobereichsanalyse

Detaillierte Erfassung aller relevanten Gefügeparameter

(Korngrößenverteilung, Korngrenzengeometrie etc.)

\section{Porenraumeigenschaften}

Charakterisierung des Porenraums durch Messen der Porosität und der Porenradienverteilung

Entfestigungszustand

Ultraschallcharakterisierung an definierten Probenkörpern

Materialsensibilität, thermisch / hygrische

Erfassung der thermisch / hygrischen Witterungssensibilität mittels Längenänderungsmessung

Wassertransportmechanismen

Ermittlung der Kapillarwirkung und der Dampfdiffusion

\section{Festigkeitsanalysen}

Erfassung der Materialkohäsion mittels Messungen der Mikrobohrhärte oder der Zugfestigkeit

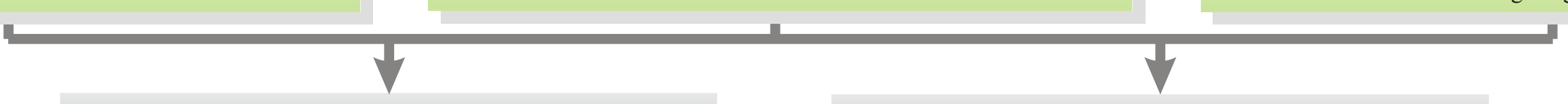

Charakterisierung des Erhaltungszustandes

Charakterisierung der Schadensprozesse

Objektbeobachtung

\section{Modul D: Monitoring}

Ultraschallmessungen

Erfassung der fortschreitenden Entfestigung mittels Messungen der Ultraschallgeschwindigkeiten

\section{Verformungsmessung}

Erfassung der fortschreitenden Öffnung von Risssystemen

\section{Modul E• Provenienz}

Gefügeanalysen

Erfassung aller relevanten makroskopischen und mikroskopischen Gefügemerkmale (Farbe,

Struktur, Korngröße etc.)

\section{Isotopenanalyse}

Einordnung in Regionale Entstehungsmilieus mittels der Isotopenverhätnisse von Sauerstoff und Kohlenstoff

Kathodolumineszenz-Mikroskopie

Ermittlung von kristallchemischen Variationen und gesteinsspezifischen Alterationsmerkmalen

\section{Konservierung}

Modul F: Voruntersuchungen

Verwitterungssimulation

Klimaschranksimulationen mit Überprüfung der Probekörper mittels Ultraschallmessungen

\section{Dehnungsverhalten, thermisch / hygrisch}

Charakterisierung der Anderung des thermischen

und hygrischen Dehnungsverhaltens

Porenraumanalysen

Charakterisierung der Veränderung des

Porenraums durch die Konservierung 
Eine detaillierte Materialcharakterisierung ist durch die Beschreibung der petrographischen, gefügekundklichen und petrophysikalischen Eigenschaften des Marmors mittels Laboruntersuchungen $\mathrm{zu}$ erreichen (Modul C). Herkömmliche Methoden, wie die Dünnschliffmikroskopie, liefern dabei wichtige Information zum Aufbau des Materials. Dabei ist für Marmore die Erfassung des Gefügeinventars von besonderer Bedeutung. Wesentliche Gefügemerkmale sind dabei die Korngröße, die Korngeometrie, die Kornformregelung und weitere besondere Vorzeichnungen wie Regelung von Zwillingen in den Kristallen und mineralisierte sowie verheilte Mikrorisse. Aber auch eine einfache qualitative Einschätzung der Texturierung ist mittels Polarisationsmikroskopie zu erreichen. Für eine weitergehende Charakterisierung des Marmors sind vielfach auch rasterelektronenmikroskopische Untersuchungen zweckmäßig. So kann an Bruchproben die Intensität chemischer Lösungsprozesse auf Korngrenzen und Spaltflächen abgeschätzt werden. Weiterhin ist eine genaue Charakterisierung des Porenraums durch Messungen der Porosität und Porenradienverteilung notwendig. Die Ermittlung des Erhaltungszustandes kann durch Ultraschallanalysen und Festigkeitsuntersuchungen erfolgen. Grundlegend für die Beurteilung der Verwitterungssensibilität ist weiterhin die Erfassung des Längenänderungsverhaltens bei thermischer und hygrischer Beanspruchung.

Aus den Ergebnissen der Untersuchungen von Modul A bis C kann der Erhaltungszustand weitgehend abgeleitet und i.d.R. der materialspezifische Entfestigungsmechanismus eingegrenzt werden. Dies dient dann als Entscheidungsgrundlage für den weiteren Umgang mit dem Objekt bzw. für die Einleitung von Maßnahmen. Bezüglich von durchzuführenden Maßnahmen kann eine weitere Charakterisierung des Marmors notwendig sein. Dies gilt vor allem für den Austausch von Teilen oder auch des gesamten Objektes. Hierzu ist häufig eine Charakterisierung der Herkunft notwendig (Modul E). Für die Provenienzanalysen eignet sich die Erfassung spezieller Mikrogefügemerkmale, wie z.B. Alterationsphänomene, die mittels Kathodolumineszenz-Mikroskopie ermittelt werden können. Ebenfalls nützlich sind Sauerstoff/Kohlenstoff-Isotopenanalysen. Diese Charakterisierung eines geeigneten Austauschmaterials kann ebenfalls die Grundlage für die Eruierung eines geeigneten Referenzmaterials für Konservierungsvorversuche sein. Dabei ist es in vielen Fällen sinnvoll, geplante Konservierungen auf ihre Materialverträglichkeit und ihre verbessernde Wirkung hinsichtlich der Witterungsresistenz zuvor in Laborversuchen zu überprüfen (Modul F). Neben Verwitterungssimulationen im Klimaschrank sind vergleichende Messungen der thermischen und hygrischen Längenänderungen des nicht konservierten und konservierten Marmors hier zweckmäßig. 


\section{Literaturverzeichnis}

AtKInson, B.K., 1987: Fracture mechanics of rock. Acad. Press, London.

Auras, M., Siedel, H. \& Weber, H., 1997: Reinigung und Steinkonservierung. In: Kiesewetter, A. \& Kiesow, G. (Hrsg.): Naturwerkstein und Umweltschutz in der Denkmalpflege: 683-716.

Battaglia, S., Franzini, M. \& Mango, F., 1993: High sensitivity apparatus for measuring linear thermal expansion: preliminary results on the response of marbles. Il Nuovo Cimento, 16: 453-461.

BIRCH, F., 1961: The velocity of compressional waves in rocks to 10 kilobars., Part 2. J. Geophys. Res., 66: 2199-2224.

BIRCH, F., 1960: The velocity of compressional waves in rocks up to 10 kilobars, Part I. J. Geophys. Res., 65: 1083-1102.

BLENKINSOP, T., 2000: Deformation microstructures and mechanisms in minerals and rocks. Kluwer Academic Publishers: 150 pp.

Brakel, J. van, Modry, S. \& Svata, M., 1981: Mercury porosimetry: State of the art. Powder technology, 29, 1-12.

Brosch, F.J., Schachner, K., Blümel, M., Fasching, A. \& Fritz, H., 2000: Preliminary investigation results on fabrics and related physical properties of an anisotropic gneiss. Journal of Structural Geology, 22: 1773-1787.

Bunge, H.J., 1982: Texture analysis in material science - mathematical methods. London.

Carmingnani, L., 1990: Crustal extension in the Northern Apennines: the transition from compression to extension in the Alpi Apuane core complex. Tectonics, 9: 1275-1303.

Carmingnani, L., Giglia, G. \& Kligfield, R., 1978: Structural evolution of the Apuane Alps: an example of continental margin deformation in the northern Apeninnes, Italy. Journal of Geology, 86: 487-504.

Clifton, J. R., 1980: Stone consolidating materials - a status report. US Department of Commerce, National Bureau of Standards, NBS Technical Note 1118:46.

DAHLGRÜN, F., 1934: Zur Altersdatierung des Vorderdevons im westsudetischen Schiefergebirge. Zeitschrift deutsche geologische Gesellschaft, 86.

DANDEKAR, D.P., 1968: Variation in the elastic constants of calcite with pressure. AGU Transactions, 49 (1): 323.

DREYER, W., 1974: Materialverhalten anisotroper Festkörper: Thermische und elektrische Eigenschaften. Springer Verlag, Wien: 295 S.

DÜRRAST, H., 1997: Gefügekundliche und gesteinsphysikalische Charakterisierung karbonatischer Reservoirgesteine - Fallbeispiele aus dem Staßfurt-Karbonat (Ca2) in Nordwestdeutschland. Cuvillier Verlag, Göttingen: 195 S. 
DUYSTER, J., 1991: Strukturgeologische Untersuchungen im Moldanubikum (Waldviertel, Österreich) und methodische Untersuchungen zur bildanalytischen Gefügequantifizierung von Gneisen. Diss., Univ. Göttingen: 185 S.

ERTAG, 1980: I marmi delle Alpi Apuane: Schede merceologiche. Regione Toscana, Nuova Grafica Fiorentina, Firenze.

Evans, A.G., 1978: Damage and microfracture from thermal expansion anisotropy in polycrystalline ceramics. Acta metall., 26: 1845-1853.

FEI, Y., 1995: Thermal expansion. In: AHRENS, T.J. (ed.): Mineral physics and crystallography - A handbook of physical constants: 29-44.

FitzNer, B. \& Snethlage, R., 1982: Einfluss der Porenradienverteilung auf das Verwitterungsverhalten ausgewählter Sandsteine. Bautenschutz und Bausanierung, Nr. 3-1982: 97-103.

FItZNER, B., 1969: Die Prüfung der Frostbeständigkeit von Naturbausteinen. Geologische Mitteilungen, 10: 205-296.

FLEISCHER, G., 2002: Beurteilung von Ultraschalluntersuchungen an Natursteinobjekten in der Denkmalpflege. Diss., TU Wien: $182 \mathrm{~S}$.

FRANZINI, M., 1995: Stones in monuments: natural and anthropogenic deterioration of marble artefacts. Eur. J. Mineral., 7: 735-743.

Frederich, J.T. \& Wong, T.F., 1986: Micromechanics of thermally induced cracking in three crustal rocks. J. Geophys. Res., 91 B12: 12,743-12,764.

Goins, E. S., Wheeler, G. S. \& WyPYSKI, M. T., 1996: Alkoxysilane film formation on quartz and calcite crystal surfaces. Proceedings $8^{\text {th }}$ International Congress on Deterioration and Conservation of Stone, Berlin, 1255-1264.

GoralczyK, P., Beeger, D. \& Materna, H., 1988: Zur Verwitterung und Konservierung von Skulpturen aus Carrara Marmor (Schlossbrückenskulpturen und HumboldtDenkmale in Berlin). Abh. des Staatl. Museums f. Min. Geol. Dresden, 35: 47-59.

GRIMM, W.D., 1999: Beobachtungen und Überlegungen zur Verformung von Marmorobjekten durch Gefügeauflockerung. Z. dt. geol. Ges., 150 No. 2: 195-236.

GRIMM, W.D. \& SchwarZ, U., 1985: Naturwerksteine und ihre Verwitterung an Münchner Bauten und Denkmälern - Überblick über eine Stadtkartierung. Arbeitsheft des Bayer. Landesamtes für Denkmalpflege, 31:28-118.

GRIMM, W.D., 1984: Zur Verwitterungen von Naturwerksteinen insbesondere bayerischer Provenienz. Geol. Bav., 86: 507-550.

Gross, C.J., Weber, K., Vollbrecht, A. \& Siegesmund, S., 1999: Cathodoluminescence and electron microprobe study of dolomitic marbles from Namibia: Evidence for hydrothermal alteration. Z. dt. geol. Ges., 150 No. 2: 333-358.

GRUNERT, S., 1988: Eine Theorie zu der an Marmorskulpturen beobachteten Innenverwitterung. Abhandlungen des Staatlichen Museums für Mineralogie und Geologie zu Dresden, 35: 95-98. 
Helming, K., 1996: Texturapproximation durch Modelkomponenten. Cuvillier Verlag, Göttingen: $119 \mathrm{~S}$.

HOLTKAMP, A., 1999: Marmorkonservierung: Vergleich ausgewählter Festigungsmittel und Applikationsmethoden an einer Probenreihe aus verwittertem Prieborner Marmor. Unveröff. Dipl. Arb., FH Potsdam: 100 S.

Honsinger, D.J. \& SASSE, H.R., 1988: Neue Wege zum Schutz und zur Substanzerhaltung von Sandsteinoberflächen unter Verwendung von Polymeren. Bautenschutz Bausanierung, 11: 205-211.

JACKSON, M.J. \& TwEETON, D.R., 1994: MIGRATOM-geophysical tomography using wavefront-migration and fuzzy constraints. Report of Investigations, U.S.B.M: 35pp.

JAHNS, E., 1995: Quantifizierte seismische Anisotropie: Ein skalenübergreifender Vergleich am Beispiel des KTB. Cuvillier Verlag, Göttingen, 107 S.

Johnson, G.C. \& WenK, H.R., 1985: Calculation of elastic anisotropy in textured marbles. In: AGU 1985 fall meeting. Eos, Transactions, American Geophysical Union. 66; 46, 1039-1085.

KESSLER, D.W., 1919: Physical and chemical tests on the commercial marbles of the United States. Technology papers of the Bureau of Standards, No. 123.

KLEBER, W., 1959: Einführung in die Kristallographie. VEB Verlag Technik, Berlin, 384 S.

KLOPFER, H., 1985: Lehrbuch der Bauphysik. Teubner Verlag, Stuttgart, 335-336.

KoblischeK, P., 1997: The consolidation of natural stone with a stone strengthener on the basis of poly-silicic-acid-ethylester. Proceedings of the $8^{\text {th }}$ International Congress on Deterioration and Conservation of Stone, Berlin, Vol. 1: 1187-1193.

KoblischeK, P.J., 1990: Protection of surfaces of natural stone and concrete through polymers. Proceedings Surface Engineering, University of Toronto.

KÖHLER, W., 1991: Untersuchungen zu Verwitterungsvorgängen an Carrara-Marmor in Potsdam-Sanssouci. Berichte zu Forschung und Praxis der Denkmalpflege in Deutschland, Steinschäden - Steinkonservierung 2: 50-5.

KRANZ, R.L., 1983: Microcracks in rocks: a review. Tectonophysics, 100 (1-3): 449-480.

LEISS, B. \& WeISS, T., 2000: Fabric anisotropy and its influence on physical weathering of different type of Carrara marbles. Leiss, B., Ullemeyer, K. \& Weber, K. (eds.) Journal of Structural Geology, Special Issue, 22: 1737-1745.

Leiss, B. \& UlLEMEYer, K., 1999: Texture characterisation of carbonate rocks and some implications for the modelling of physical anisotropies, derived from idealized texture types. Z. dt. geol. Ges., 150: 259-274.

LEISS, B., 1996: Strukturbezogene Textur- und Korngefügeuntersuchungen duktil deformierter Dolomitgesteine am Südwestrand des Damara Orogens (Namibia): Mechanismen der Texturbildung und ihre kinematische Bedeutung. Geotekt. Forsch., 84: 1-130. 
Lindner, H., Pretzschner, C. \& Rost, L., 1999: Ultraschalluntersuchungen an Bauwerken. Z. dt. geol. Ges., 150: 375-386.

LORENZ, H.G. \& IBACH, H.W., 1999: Marble conservation by Ibach-Total-Impregnation Process: Quality control and optimisation by microscopic and petrophysical data. Zeitschrift Deutsche Geologische Gesellschaft, 150: 397-406.

MARShall, D.J., 1988: Cathodoluminescence of geological materials. Unwin Hyman, London-Sydney-Wellington: $156 \mathrm{pp}$.

Mcskimin, H.J., Anreatch, P. \& Thurston, R.N., 1965: Elastic moduli of quartz versus hydrostatic pressure at $25^{\circ} \mathrm{C}$ and $-195.8^{\circ} \mathrm{C}$. J. Appl. Phys., 36: 1624-1632.

Moore, A.C., 1970: Descriptive terminology for textures of rocks in granulite facies terrains. Lithos, 3: 123-127.

MuRAWSKI, H., 1992: Geologisches Wörterbuch, 9. Auflage. Enke Verlag, Stuttgart: 250 S.

NEUSER, R.D., 1988: Zementstratigraphie und Kathodolumineszenz des Korallenooliths (Malm) im Südniedersächsischen Bergland. Bochumer geol. u. geotechn. Arb., 32: $172 \mathrm{~S}$.

NOLET, G., 1987: Seismic tomography. Reidel Verlag, Dordrecht: 386pp.

NuR, A. \& Simmons, G., 1969: The effect of saturation on velocity in low porous rocks. Earth and Planetary Science Letters, 7: 183-193.

O'Connell, R.J. \& Budiansky, B., 1974: Seismic velocities in dry and saturated cracked solids, J. Geophys. Res., 79(35): 5412-5426.

Ondrasina, J., Kirchner, D. \& Siegesmund, S., 2002: Frost-/Thaw cycles and their influence on marble deterioration: a long-term experiment. In: Siegesmund, S., Weiss, T. \& Vollbrecht, A. (eds.): Natural stones, weathering phenomena, conservation strategies and case studies. Geological Society Special Publication, 205, 1-10.

PAsschier, C.W. \& Trouw, R.A.J., 1996: Microtectonics. Springer Verlag, Berlin.

Peck, L., Barton, C.C. \& Gordon, R.B., 1985: Microstructure and the resistance of rock to tensile fracture. J. Geophys. Res., 90 B 13: 533-546.

PIELOW, H.U., 1997: Korrodierte Mineralgrenzflächen als Indikatoren für die Migration salinarer Fluide im Kristallin am Beispiel des KTB. Göttinger Arb. Geol. Paläont., 71: $55 \mathrm{~S}$.

PoschloD, K., 1989: Das Wasser im Porenraum kristalliner Naturwerksteine und sein Einfluß auf die Verwitterung. Münchner Geowissenschaftliche Abhandlungen, 7: $62 \mathrm{~S}$.

RADON, J., 1917: Über die Bestimmung von Funktionen durch ihre Integralwerte längs gewisser Mannigfaltigkeiten. Ber. Verh. Sächs. Akad. Wiss., 69: 262-277.

Rasolofosaon, P.N.J., Rabbel, W., Siegesmund, S. \& Vollbrecht, A., 2000: Characterization of crack distribution: fabric analysis versus ultrasonic inversion. Geophys. J. Int., 141: 413-424. 
REEDER, R.J., 1991: An overview of zoning in carbonate minerals. In: Barker, C.E. \& Kopp, O.C. (eds.): SEPM Short Course 25, Luminescence Microscopy and spectroscopy: Qualitative and quantitative applications.

RichteR, D., 2000: Modellhafte Schadensanalyse von Trommelsäulen des Marmorpalais Potsdam. Unveröff. Dipl. Arb., Univ. Göttingen: 67 S.

Rohatsch, A., Nimmrichter, J. \& Chalupar, I., 2000: Physical properties of fine grained marble before and after conservation. Proceedings 9th International congress on deterioration and conservation of stone, Venice, Volume 1: 215-223.

Ruedrich, J. Weiss, T. \& Siegesmund, S., 2002: Thermal behaviour of weathered and consolidated marbles. In: Siegesmund, S., Weiss, T. \& Vollbrecht, A. (eds.): Natural stones, weathering phenomena, conservation strategies and case studies. Geological Society Special Publication, 205: 255-271.

Ruedrich, J., WeISS, T. \& SiegeSmund, S., 2001a: Deterioration characteristics of marbles from the Marmorpalais Potsdam (Germany): a compilation. Zeitschrift Deutsche Geologische Gesellschaft, 152/2-4: 637-664.

Ruedrich, J., Siegesmund, S. \& Richter, D., 2001b: Marble Columns and their State of weathering: structural evidences and Ultrasonic Tomography. Zeitschrift Deutsche Geologische Gesellschaft, 152/2-4: 665-680.

SAGE, J. D., 1988: Thermal microfracturing of marble. In: Marinos, P. \& Koukies, G. (eds.) Engineering Geology of Ancient Works, Balkema, Rotterdam, 1013-1018.

SANDER, B., 1930: Gefügekunde der Gesteine: mit besonderer Berücksichtigung der Tektonite. Springer Verlag, Wien: 346 S.

Schild, M., Siegesmund, S., Vollbrecht, A. \& Mazurek, M., 2001: Characterization of granite matrix porosity and pore-space geometry by in situ and laboratory methods. Geophysical Journal International, 146: 111-125.

Siegesmund, S., Vollbrecht, A., Chlupac, T., Nover, G., Dürrast, H., Müller, J. \& WEBER, K., 1993: Fabric-controlled anisotropy of petrophysical properties observed in KTB-core samples. Sci. Drilling, 4: 31-54.

SiEgesmund, S., 1996: The significance of rock fabrics for the geological interpretation of geophysical anisotropies. Geotekt. Forsch., 85: $123 \mathrm{~S}$.

Siegesmund, S., Vollbrecht, A., Ullemeyer, K., Weiss, T. \& Sobott, R., 1997: Application of geological fabric analyses for the characterization of natural building stones - case study Kauffung marble (in German). International Journal for Restoration of Buildings and Monuments, 3: 269-292.

Siegesmund, S., Weiss T., Vollbrecht A. \& Ullemeyer K., 1999: Marble as a natural building stone: rock fabrics, physical and mechanical properties. Z. dt. geol. Ges., 150: 237-257.

Siegesmund, S., Pretzschner, C., Ruedrich, J., Lindner, H., Weiss, T., Richter, I., RICHTER, D. \& WOYDE, M., 2000a: Deterioration characteristics of columns from the 
Marmorpalais Potsdam (Germany) by ultrasonic-tomography. Proceedings $9^{\text {th }}$ International congress on deterioration and conservation of stone, Venice, Volume 2: 145-153.

Siegesmund, S., Weiss, T. \& TschegG, E.K., 2000b: Control of marble weathering by thermal expansion and rock fabrics.- Proceedings 9th International congress on deterioration and conservation of stone, Venice, Volume 1: 205-214.

Siegesmund, S., Ullemeyer, K., Weiss, T. \& TschegG, E.K. 2000c: Physical weathering of marbles caused by anisotropic thermal expansion. International Journal of Earth Science, 89: 170-182.

Siegesmund, S., Weiss, T. \& RUEDRICH, J., 2001: Schadensanalyse von Architekturteilen am Marmorpalais Potsdam. Zeitschrift Naturstein, Ausgabe 5: 92-101.

SIMON, S., 2001: Zur Verwitterung und Konservierung kristallinen Marmors. Diss., Univ. München: 256S.

SkInNER, B.J., 1966: Thermal expansion. In: CLARK, S.P. (ed.): Handbook of physical constants. Geological Society of America, 97: 75-96.

SkowroneK, A., 2001: Der Kauffunger Kalkstein (Bober-Katzbach Gebirge, Polen): Petrographie, Fossilinventar, Stratigraphie. Diss., Univ. Hannover: 112 S.

SkowroneK, A. \& Steffahn, J., 2000: The age of the Kauffung Limestone (W Sudetes, Poland) - a revision due to new discovery of microfossils. N. Jb. Geol. Paläont. Mh. 2: 65-82.

SKrotZKI, W., 1994: Mechanisms of texture development in rocks. In: Bunge, H.J., Siegesmund, S., Skrotzki, W. \& Weber, K. (eds.): Textures of geological materials, DGM-Press.

Snethlage, R., EtTl, H. \& SATtler, L., 1999: Ultraschalluntersuchungen an PMMAgetränkten Marmorskulpturen. Z. dt. geol. Ges., 150: 387-396.

SNethlage, R., 1984: Steinkonservierung: Arbeitshefte des Bayerischen Landesamtes für Denkmalpflage, 22: $203 \mathrm{~S}$.

Strohmeyer, D. \& Siegesmund, S., 2002: Anisotropic technical properties of building stones and their development due to fabric changes. In: Siegesmund, S., Weiss, T. \& Vollbrecht, A. (eds.): Natural stones, weathering phenomena, conservation strategies and case studies. Geological Society Special Publication, 205: 107-127.

TRÖGER, W.E., 1967: Optische Bestimmung der gesteinsbildenden Minerale: Teil 2. E. Schweitzerbart'sche Verlagsbuchhandlung Stuttgart.

TscheGG, E.K., Widhalm, C. \& Eppensteiner, W., 1999: Ursachen mangelnder Formbeständigkeit von Marmorplatten. Z. dt. Geol. Ges., 150 No. 2: 283-297.

Turner, F.J., Griggs, D.T. \& HEARD, H., 1954: Experimental deformation of calcite crystals. Geol. Soc. America Bull., 65: 883-934. 
Tweeton, D.R., Jackson, M.J. \& Roessler, K.S., 1992: BOM-CRATR-A Curved-Ray Tomographic Computer Program for Geophysical Application. Bureau of Mines RI 9411: 39pp.

VAughan, M.T. \& GugGenheim, S., 1986: Elasticity of muscovite and its relationship to crystal structure. J. Geophys. Res., 91: 4657-4664.

VoIGT, W., 1928: Lehrbuch der Kristallphysik. Teubner Verlag, Leipzig.

Vollbrecht, A., Dürrast, H. \& Weber, K., 1994: Eine einfache Methode zur dreidimensionalen Quantifizierung von Rißschardichten auf der Basis von U-TischMessungen. KTB-Report, 94-2: B89.

Vollbrecht, A., Rust, S. \& Weber, K., 1991: Development of microcracks in granites during cooling and uplift: examples from the Variscan basement in NE-Bavaria, Germany. Journal of Structural Geology, 13:787-799.

WeBER, J., 1985: Natural and artificial weathering of Australian building stones due to air pollution. Proc. Of the $5^{\text {th }}$ International Congress on Deterioration and Conservation of Stone, Lausanne: 527-535.

Weiss, T., Fuller, E. \& Siegesmund, S., 2002: Thermal stresses in calcite and dolomite marbles quantified by finite element modelling. In: Siegesmund, S., Weiss, T. \& Vollbrecht, A. (eds.): Natural stones, weathering phenomena, conservation strategies and case studies. Geological Society Special Publication, 205, 81-94.

Weiss T., Rasolofosaon, P.N.J. \& Siegesmund, S., 2001: Thermal microcracking in Carrara marble. Z. dt. geol. Ges., 152/2-4: 621-636.

Weiss T., Siegesmund, S. \& Rasolofosaon, P.N.J., 2000: The relationship between deterioration, fabric, velocity and porosity constraint. Proceedings $9^{\text {th }}$ International congress on deterioration and conservation of stone, Venice, Vol. 1: 215-223.

Weiss, T., Leiss, B., Oppermann, H. \& Siegesmund, S., 1999: Microfabric of fresh and weathered marbles: Implications and consequences for the reconstruction of the Marmorpalais Potsdam. Z. dt. Geol. Ges., 150: 313-332.

WEISS, T., 1998: Gefügeanisotropie und ihre Auswirkung auf das seismische Erscheinungsbild: Fallbeispiele aus der Lithosphäre Süddeutschlands. Geotektonische Forschungen, 91: $156 \mathrm{~S}$.

Widhalm, C., EpPensteiner, W. \& TschegG, E., 1999: Meßmethoden zur Beschreibung von Gefügeanisotropien in Marmor. Z. dt. geol. Ges., 150/2: 275-281.

Widhalm, C., Tschegg, E. \& Eppensteiner, W., 1996: Anisotropic thermal expansion causes deformation of marble cladding. J. of Performance of Constructed facilities, ASCE, 10: 5-10.

WiHR, R., 1995: Fünfzehn Jahre Acrylharzvolltränkung in Bayern. Arbeitsblätter Stein, 9: 3240.

WiHR, R., 1980: Restaurierung von Steindenkmälern: ein Handbuch für Restauratoren, Architekten, Steinbildhauer und Denkmalpfleger. Callwey Verlag, München. 
Wimmenauer, W., 1985: Petrographie der magmatischen und metamorphen Gesteine. Enke Verlag Stuttgart: $381 \mathrm{~S}$.

Zeisig, A., Siegesmund, S. \& Weiss, T. (2002): Thermal expansion and its control on the durability of marbles. In: Siegesmund, S., Weiss, T. \& Vollbrecht, A. (eds.): Natural stones, weathering phenomena, conservation strategies and case studies. Geological Society Special Publication, 205, 65-80.

Zezza, U., Mssara, E.P., Massa, V. \& Venchiarutti, D., 1985: Effect of temperature on intergranular decohesion of the marbles. Proceedings $5^{\text {th }}$ Int. Congr. on Deterioration and Conservation of Stone, Lausanne, Vol. 1: 131-140. 


\section{Danksagung}

Grundlage für die Realisierung der vorliegenden Arbeit war ein Promotionsstipendium der Deutschen Bundesstiftung Umwelt. Mein Dank gilt allen Mitarbeiterinnen und Mitarbeitern der Stiftung für Ihre Unterstützung.

Die Arbeit entstand auf Anregung und unter Betreuung von Prof. Dr. Siegfried Siegesmund (Geowissenschaftliches Zentrum der Universität Göttingen). Das Korreferat übernahm Prof. Dr. Rolf Snethlage vom Bayerischen Landesamt für Denkmalpflege. Beiden danke ich für ihr stetes Interesse an der Arbeit und für die ertragreichen Diskussionen.

Mein besonderer Dank gilt weiterhin

- Prof. Dr. Elmar Tschegg und seinen Mitarbeiten von der Angewandten Physik der TU Wien für ihre Hilfsbereitschaft und für die Bereitstellung des Dilatometers

- Dr. Wolf Ibach und Peter J. Koblischek für die Unterstützung bei den Konservierungen

- Dr. Robert Sobott und Dr. Artur Skowronek für eine nicht nur erfolgreiche sondern auch vergnügliche Exkursion und Probennahme in Polen

- Dr. Axel Vollbrecht, Dr. Till Heinrichs und Dr. Klaus Ullemeyer für die fachliche Unterstützung bei den Gefügeanalysen

- Dr. Till Popp für seine unermüdlichen Anstrengungen bei Konservierungen der Proben im Labor

- Detlef Röper und Herrn Höllriegel für die Hilfe bei der weiteren Probenbeschaffung

- Ina Richter, Maureen Woyde und Carsten Pretzschner für die gute Zusammenarbeit bei der Ultraschalltomographie

- Dr. Thomas Weiss, Daniel Strohmeyer und Andreas Koch für die gute Zusammenarbeit in der Arbeitsgruppe Tektoniklabor

- Dr. Helmut Dürrast für die Unterstützung bei den petrophysikalischen Analysen und Dr. Eberhard Jahns für seine Hilfe bei den Festigkeitsuntersuchungen

- Michael Sossnitza, Lothar Laake, Günther Tondock und Harald Tonn für ihre sehr kompetente technische Unterstützung

- Wolfgang Hoffmann und Daniela Boßmeyer für den Beistand bei den Korrekturen

Tanja Eggers danke ich aus unzähligen Gründen, in erster Linie dafür, dass ich sie stets bekochen darf. 


\section{Lebenslauf}

Geboren am 20 Januar 1969 in Witzenhausen

Staatsangehörigkeit deutsch

\section{Hochschulausbildung und wissenschaftlicher Werdegang}

Seit 01/2003 Wissenschaftliche Hilfskraft mit Examen an der Georg-AugustUniversität Göttingen; Mitarbeit im Projekt:

- Das Marktor von Milet: Schadensanalyse und modellhafte Sanierungskonzepte, DBU

01/2000 - 06/2003 Promotion mit dem Titel „Gefügekontrollierte Verwitterung natürlicher und konservierter Marmore" gefördert durch ein Promotionsstipendium der Deutschen Bundesstiftung Umwelt

1999 Forschungsaufenthalt an der TU Wien gefördert durch ein Stipendium des Österreichischen Austauschdienstes

1998 - 1999 Wissenschaftliche Hilfskraft mit Examen an der Georg-AugustUniversität Göttingen; Mitarbeit in den Projekten:

- Schadensanalyse und modellhafte Sanierung von Architekturteilen am Marmorpalais, Potsdam, DBU

- Mikrostrukturelle Untersuchungen an Konglomeraten der Witwatersrand Lagerstätte, BGR

- und Leitung des Erstsemester-Tutoriums WS 98/99 u. WS 99/00

1994 - 1998 Wissenschaftliche Hilfskraft an der Georg-August-Universität Göttingen; Mitarbeit in den Projekten:

- Gefügekundliche Untersuchungen an Geröllquarzen des Witwatersrand, BGR

- Gefügekundliche und petrographische Untersuchungen an Geröllquarzen und polymineralischen Gesteinsfragmenten von goldführenden präkambrischen Konglomeraten Afrikas, BGR

- Gesteinsmagnetische Untersuchungen zur Beschreibung der Inplatznahme des Adara Plutons

10/1990 - 04/1998 Studium im Diplomfach Geologie/Paläontologie an der GeorgAugust-Universität Göttingen, Vordiplom 1992 